\title{
First Responders Guide to Computer Forensics
}

Richard Nolan

Colin O'Sullivan

Jake Branson

Cal Waits

March 2005

CERT Training and Education

HANDBOOK

CMU/SEI-2005-HB-001 

= CarnegieMellon

드 Software Engineering Institute

Pittsburgh, PA 15213-3890

\section{First Responders Guide to Computer Forensics}

CMU/SEI-2005-HB-001

Richard Nolan

Colin O'Sullivan

Jake Branson

Cal Waits

March 2005

CERT Training and Education

Unlimited distribution subject to the copyright. 
This report was prepared for the

SEI Joint Program Office

$\mathrm{ESC} / \mathrm{XPK}$

5 Eglin Street

Hanscom AFB, MA 01731-2100

The ideas and findings in this report should not be construed as an official DoD position. It is published in the interest of scientific and technical information exchange.

FOR THE COMMANDER

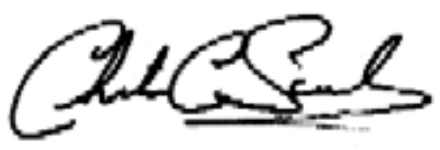

Christos Scondras

Chief of Programs, XPK

This work is sponsored by the SEI FFRDC primary sponsor and the Commander, United States Army Reserve (USAR) Information Operations Command and USAR EIO. The Software Engineering Institute is a federally funded research and development center sponsored by the U.S. Department of Defense.

Copyright 2005 Carnegie Mellon University.

NO WARRANTY

THIS CARNEGIE MELLON UNIVERSITY AND SOFTWARE ENGINEERING INSTITUTE MATERIAL IS FURNISHED ON AN "AS-IS" BASIS. CARNEGIE MELLON UNIVERSITY MAKES NO WARRANTIES OF ANY KIND, EITHER EXPRESSED OR IMPLIED, AS TO ANY MATTER INCLUDING, BUT NOT LIMITED TO, WARRANTY OF FITNESS FOR PURPOSE OR MERCHANTABILITY, EXCLUSIVITY, OR RESULTS OBTAINED FROM USE OF THE MATERIAL. CARNEGIE MELLON UNIVERSITY DOES NOT MAKE ANY WARRANTY OF ANY KIND WITH RESPECT TO FREEDOM FROM PATENT, TRADEMARK, OR COPYRIGHT INFRINGEMENT.

Use of any trademarks in this report is not intended in any way to infringe on the rights of the trademark holder.

Internal use. Permission to reproduce this document and to prepare derivative works from this document for internal use is granted, provided the copyright and "No Warranty" statements are included with all reproductions and derivative works.

External use. Requests for permission to reproduce this document or prepare derivative works of this document for external and commercial use should be addressed to the SEI Licensing Agent.

This work was created in the performance of Federal Government Contract Number F19628-00-C-0003 with Carnegie Mellon University for the operation of the Software Engineering Institute, a federally funded research and development center. The Government of the United States has a royalty-free government-purpose license to use, duplicate, or disclose the work, in whole or in part and in any manner, and to have or permit others to do so, for government purposes pursuant to the copyright license under the clause at 252.227-7013.

For information about purchasing paper copies of SEI reports, please visit the publications portion of our Web site (http://www.sei.cmu.edu/publications/pubweb.html). 


\section{Contents}

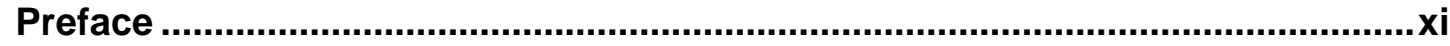

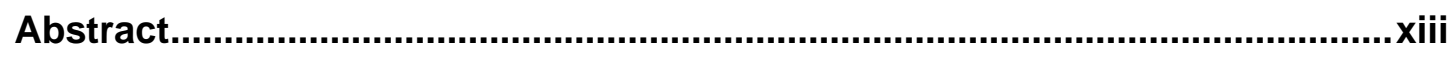

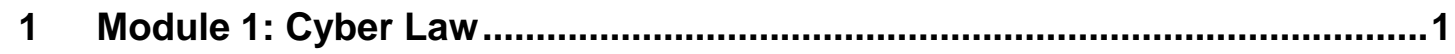

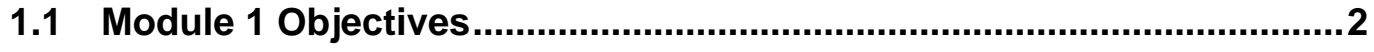

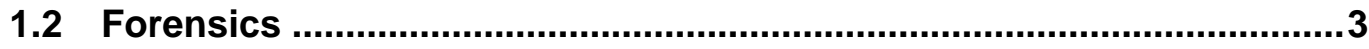

1.2.1 Computer Forensics ………….............................................

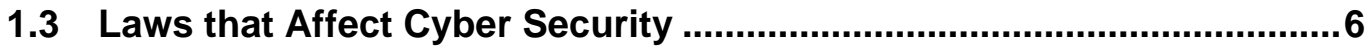

1.4 Legal Governance Related to Monitoring and Collection.......................9

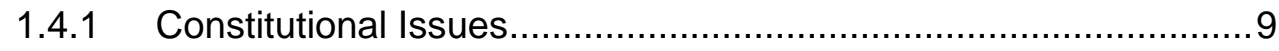

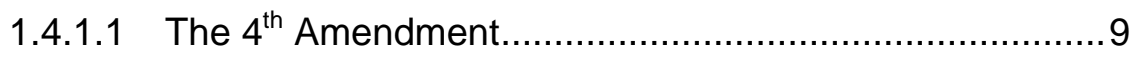

1.4.1.2 The $5^{\text {th }}$ Amendment...............................................11

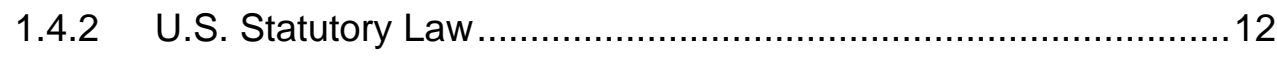

1.4.2.1 Wiretap Act/Electronic Communications Privacy Act....15

1.4.2.2 Pen Registers and Trap and Trace Devices .................18

1.4.2.3 Stored Wired and Electronic Communications Act .......21

1.5 Legal Governance Related to Admissibility (Federal Rules of

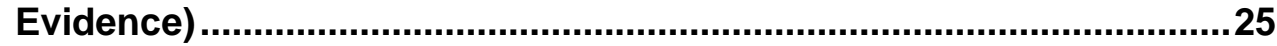

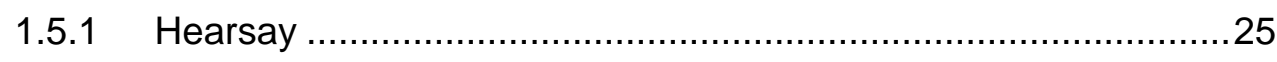

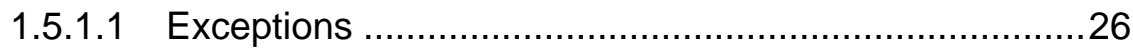

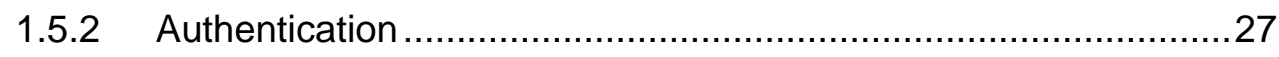

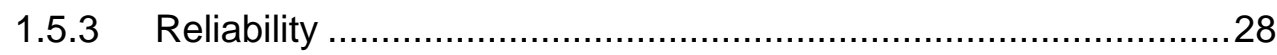

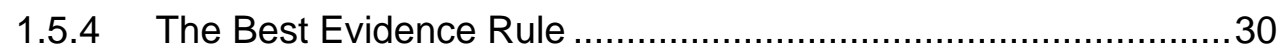

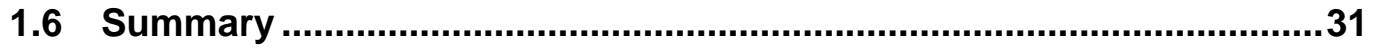

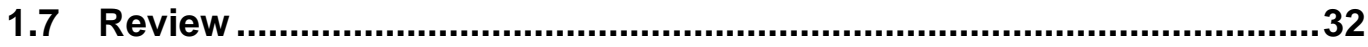

2 Module 2: Understanding File Systems and Building a First Responder

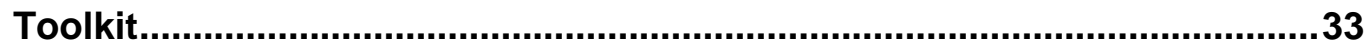

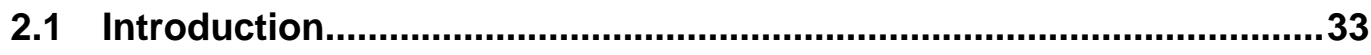

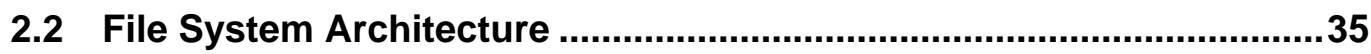

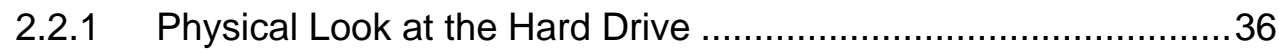

2.2.2 Types of Hard Drive Formatting................................................37

2.2.3 Importance of File Systems ......................................................38

2.2.4 Understanding Windows File Structure ……………………......39 
2.2.5 FAT: File Allocation Table ............................................. 41

2.2.6 NTFS: New Technology File System........................................ 43

2.2.7 Windows Registry................................................................ 46

2.2.8 Swap File, Slack, and Unallocated Space............................... 47

2.2.8.1 Swap File ........................................................... 47

2.2.8.2 Slack Space ................................................... 47

2.2.8.3 Unallocated Space ............................................. 48

2.2.9 Linux File System Basics..................................................... 49

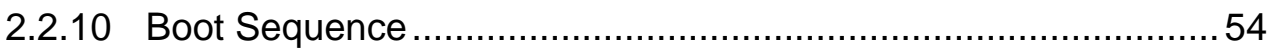

2.2.11 Commonly Used Terms .................................................... 56

2.2.12 Forensically Sound Duplication ........................................ 57

2.2.13 Duplication Tools ................................................................... 58

2.2.14 Wiping Storage Devices .................................................... 59

2.2.15 DoD Directive 5220-22M ..................................................... 60

2.2.16 Hard Drives.................................................................... 61

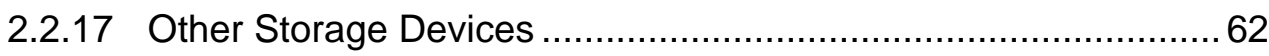

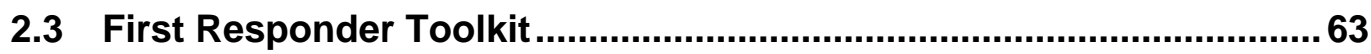

2.3.1 Statically- vs. Dynamically-Linked Tools .................................6 64

2.3.2 Problems with Dynamically-Linked Executables .......................66 66

2.3.3 Methodology for a Creating First Responder Toolkit ..................68

2.3.3.1 Create a Forensic Tool Testbed ..............................69 69

2.3.3.2 Document the Testbed .......................................... 72

2.3.3.3 Document and Set Up the Forensic Tools................... 73

2.3.3.4 Test the Tools .................................................... 75

2.3.3.5 Benefits of Proper Tool Testing ............................... 80

2.3.3.6 NIST Methodology .......................................... 81

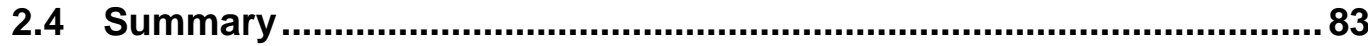

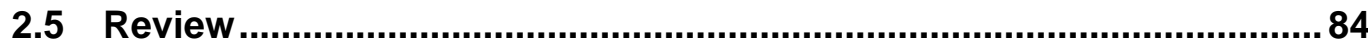

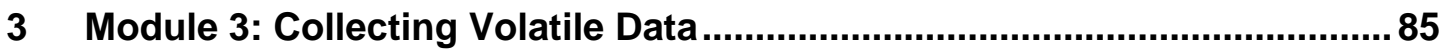

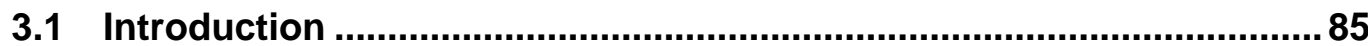

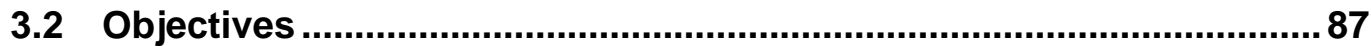

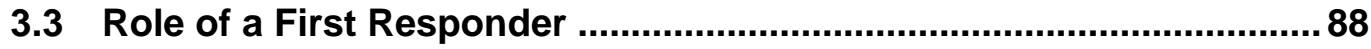

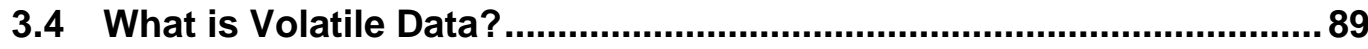

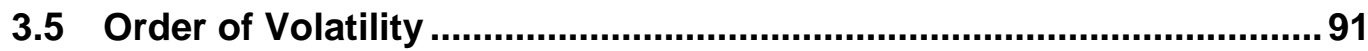

3.6 Why is Volatile Data Important? ......................................................92

3.7 Common First Responder Mistakes ...................................................93

3.8 Volatile Data Collection Methodology ..................................................94

3.8.1 Step 1: Incident Response Preparation .................................... 95

3.8.2 Step 2: Incident Documentation ........................................ 96

3.8.2.1 Incident Profile .............................................. 96 
3.8.2.2 Forensic Collection Logbook .................................99

3.8.2.3 First Responder Toolkit Logbook .............................97

3.8.3 Step 3: Policy Verification ................................................ 98

3.8.4 Step 4: Volatile Data Collection Strategy.................................. 99

3.8.5 Step 5: Volatile Data Collection Setup......................................100

3.8.5.1 Establish a Trusted Command Shell........................100

3.8.5.2 Establish a Method for Transmitting and Storing the Collected Information ............................................100

3.8.5.3 Ensure the Integrity and Admissibility of the Forensic Tool Output ......................................................... 101

3.8.6 Step 6: Volatile Data Collection Process ..............................102

3.9 Types of Volatile Information ..........................................................103

3.9.1 Volatile System Information ............................................104

3.9.1.1 System Profile ................................................. 105

3.9.1.2 Current System Date and Time and Command History

3.9.1.3 Current System Uptime ....................................111

3.9.1.4 Running Processes ............................................. 113

3.9.1.5 Open Files, Startup Files, and Clipboard Data...........122

3.9.1.6 Logged On Users ............................................. 135

3.9.1.7 DLLs or Shared Libraries ..................................... 143

3.9.2 Volatile Network Information.............................................. 147

3.9.2.1 Open Connections and Ports ...................................148

3.9.2.2 Routing Information......................................... 154

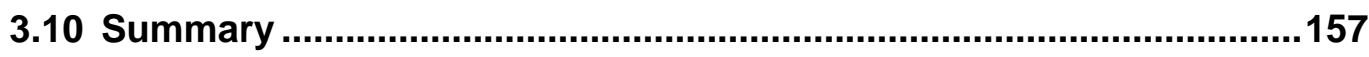

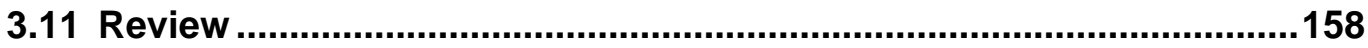

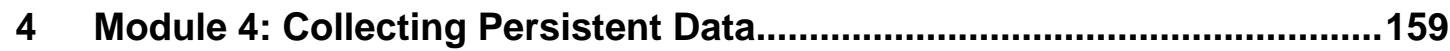

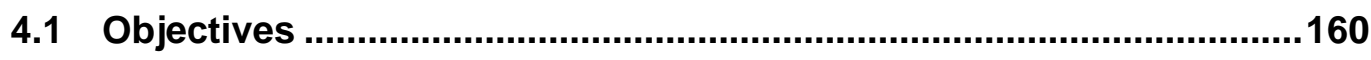

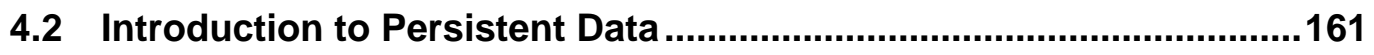

4.2.1 What Is Persistent Data? ................................................. 161

4.2.2 Why is Persistent Data Important? .....................................161

4.2.3 What Problems Exist in Investigating Persistent Data? .............161

4.3 Responding to a Security Event ...................................................163

4.3.1 Consequences of Responses....................................... 164

4.4 Basic Building Blocks of Disk Storage ..........................................166

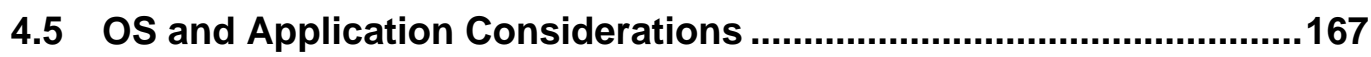

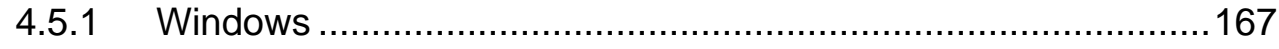

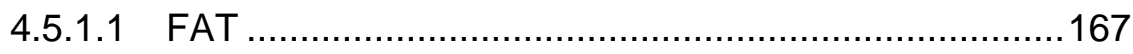

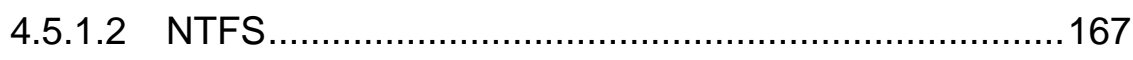

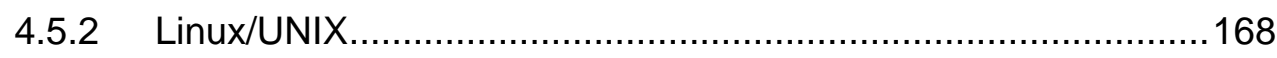

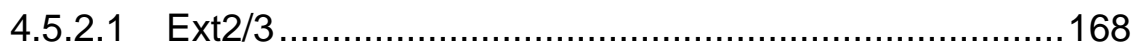


4.5.3 Operating Systems .................................................. 168

4.6 Collecting Forensic Evidence ........................................................... 169

4.6.1 To Shut Down or Not to Shut Down.................................... 171

4.6.2 Creating a Disk Image Using dd...................................... 172

4.7 Persistent Data Types.................................................................... 173

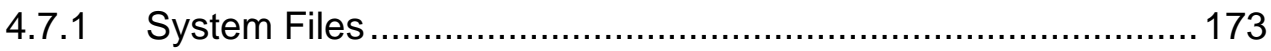

4.7.1.1 Windows ......................................................... 173

4.7.1.2 UNIX/Linux..................................................... 174

4.7.2 Temp Files.................................................................... 176

4.7.3 Web Artifacts ................................................................. 178

4.7.3.1 Windows vs. Linux ........................................... 178

4.7.3.2 IE Default Locations............................................ 178

4.7.3.3 Alternative Browsers ....................................... 181

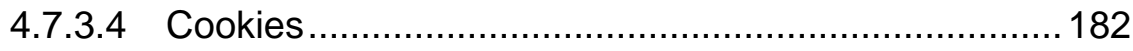

4.7.4 File Recovery .............................................................. 183

4.7.4.1 Deleted Data........................................................ 183

4.7.4.2 Slack Space ....................................................... 184

4.7.4.3 Swap Files .................................................... 184

4.7.4.4 Unallocated Space.......................................... 184

4.7.4.5 Partial Files .......................................................... 184

4.7.4.6 Windows Artifacts ......................................... 185

4.7.5 Hidden Files.................................................................. 186

4.8 Recovering a Deleted Email ............................................................ 187

4.9 Tools for Accessing Persistent Data.................................................. 188

4.9.1 Windows ...................................................................... 188

4.9.1.1 Command-Line Tools ..................................... 188

4.9.1.2 GUI-Based Utilities ........................................... 188

4.9.1.3 Commercial.................................................. 189

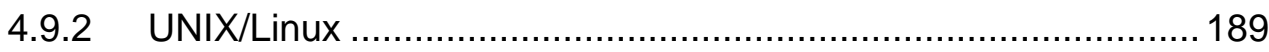

4.9.2.1 Command-Line Tools ......................................... 189

4.9.2.2 GUI-Based Utilities ........................................ 189

4.9.2.3 Freeware ...................................................... 189

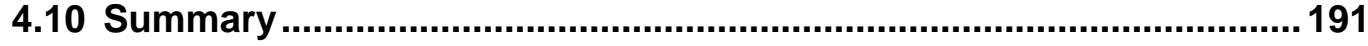

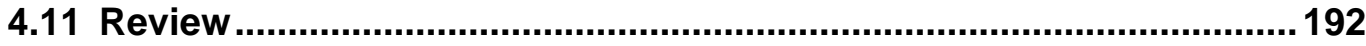

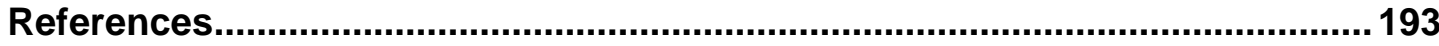




\section{List of Figures}

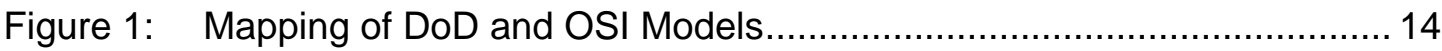

Figure 2: Logical Layout of the FAT32 File System ....................................... 42

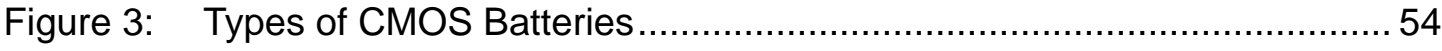

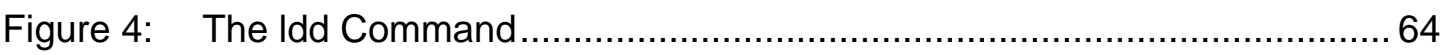

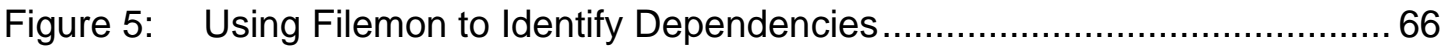

Figure 6: Performing a Cryptographic Hash of Installed DLLs ........................ 70

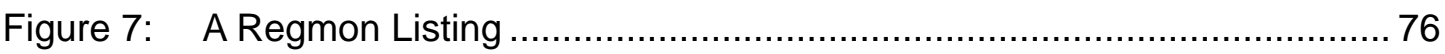

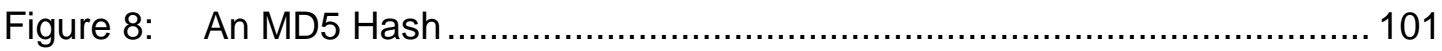

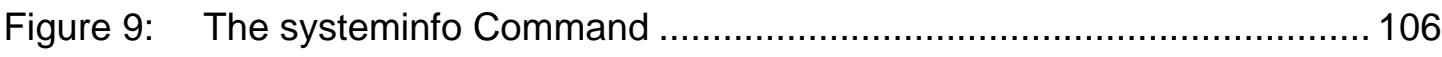

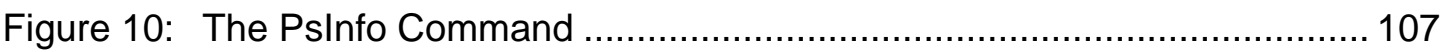

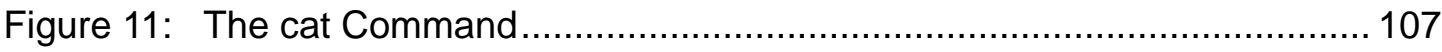

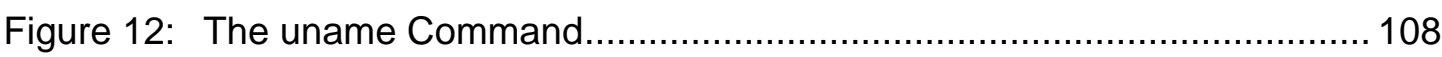

Figure 13: date and time Commands Used with netstat.................................. 109

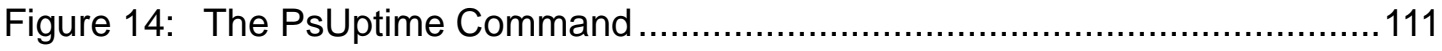

Figure 15: The net statistics Command ............................................... 112

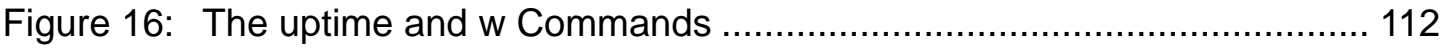

Figure 17: Using netstat -ab to Determine Process Executable Image .............. 115

Figure 18: Using ListDLLs to Determine Command Line ............................. 115

Figure 19: Using PsList to Determine How Long a Process Has Been Running .. 115 
Figure 20: Using PsList to Determine How Much Virtual Memory a Process Is Using

Figure 21: Using ListDLLs to Discover the Currently Loaded DLLs for a Process.116

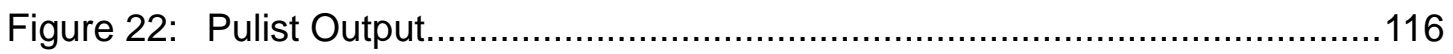

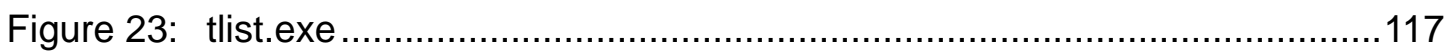

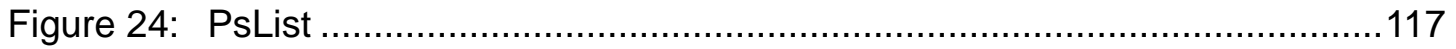

Figure 25: A Process Memory Dump …............................................... 118

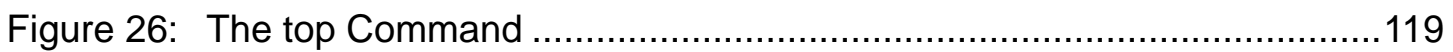

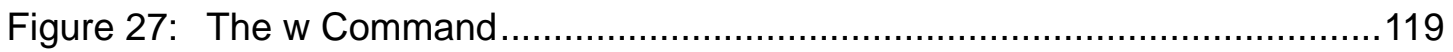

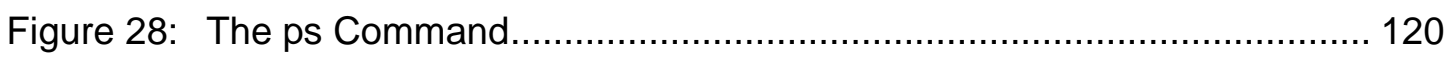

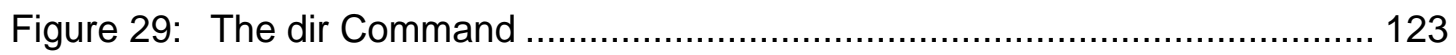

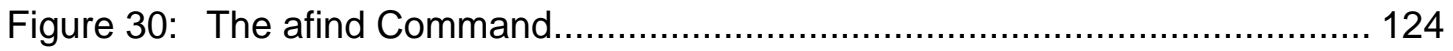

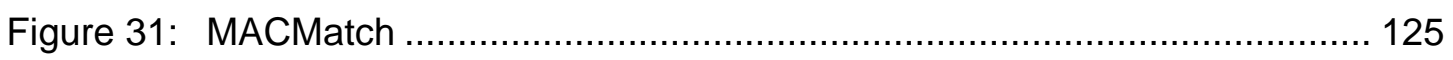

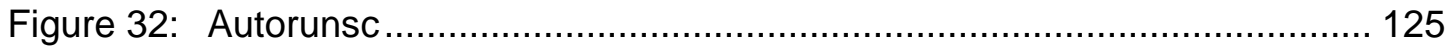

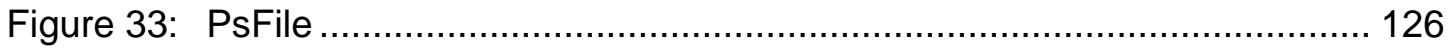

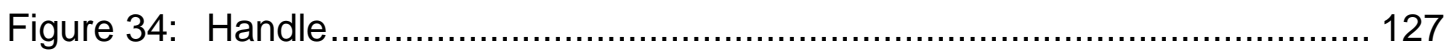

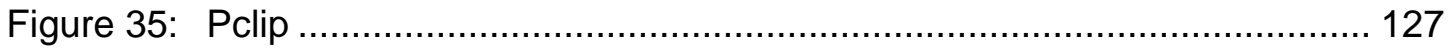

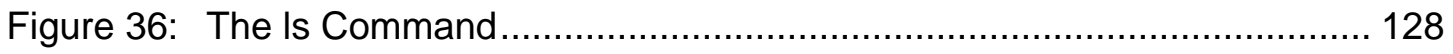

Figure 37: Using Is - alct to Show Last Modification Date and Time .................. 129

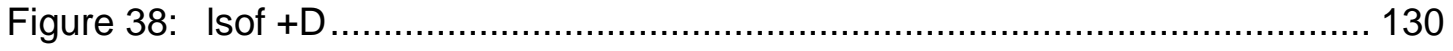

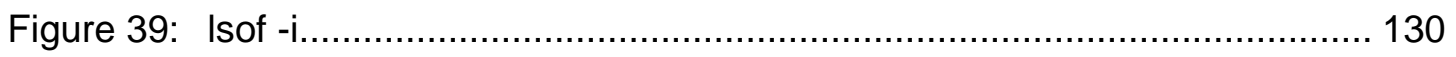

Figure 40: The find Command......................................................... 130

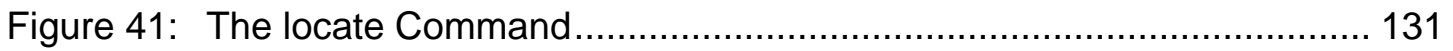




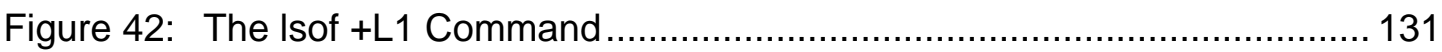

Figure 43: The chkconfig -list Command ............................................... 133

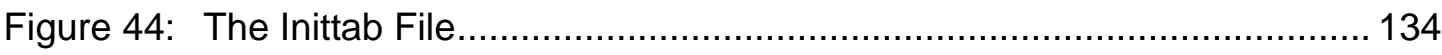

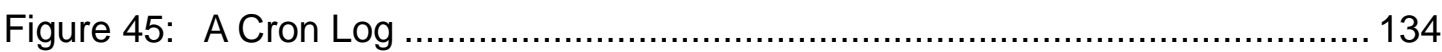

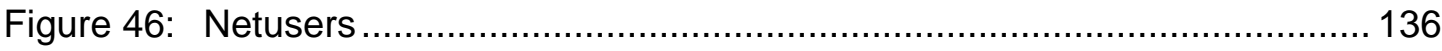

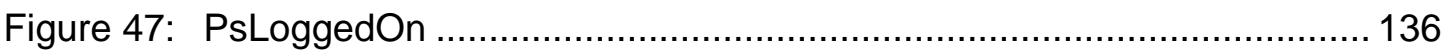

Figure 48: The net user Command ........................................................... 137

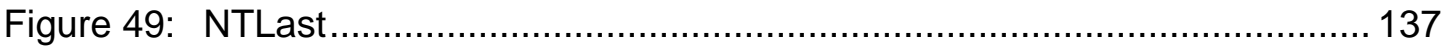

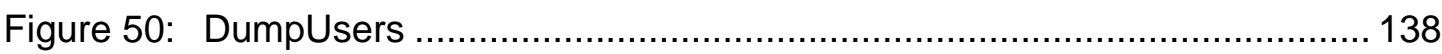

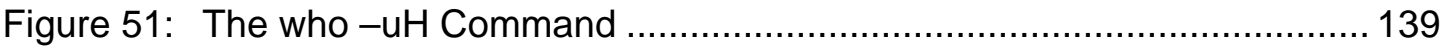

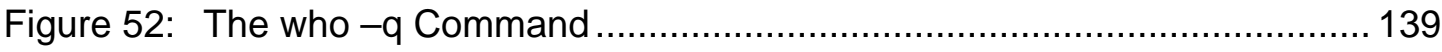

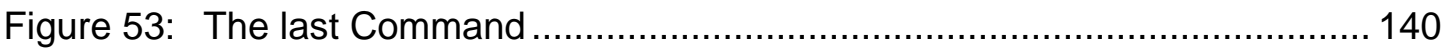

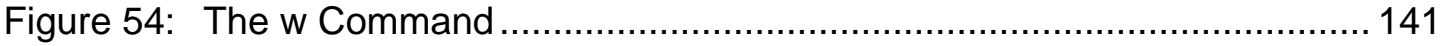

Figure 55: The cat /etc/passwd Command .............................................. 141

Figure 56: The cat shadow Command …................................................... 142

Figure 57: Using ListDLLs Without Options to Produce a List of DLLs ................ 144

Figure 58: Using ListDLLs to Examine a Suspected Rogue Process.................. 145

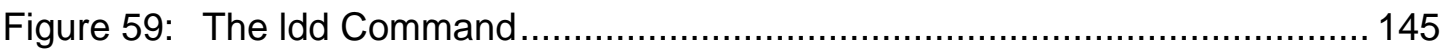

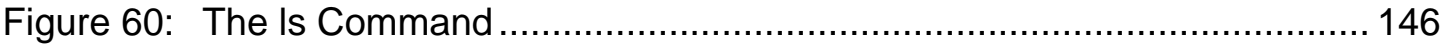

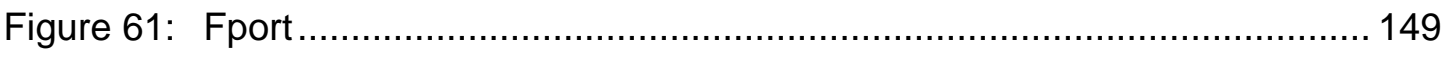

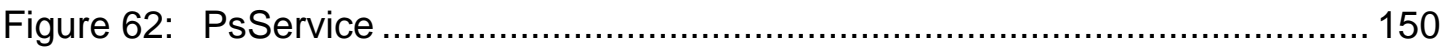

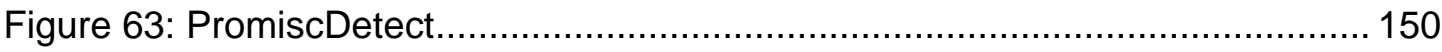

Figure 64: The netstat -anb Command ...................................................... 151

CMU/SEI-2005-HB-001 vii 


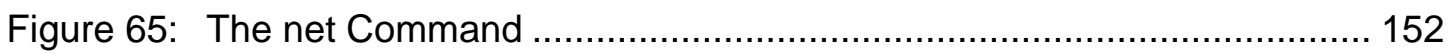

Figure 66: The netstat -anp Command................................................. 152

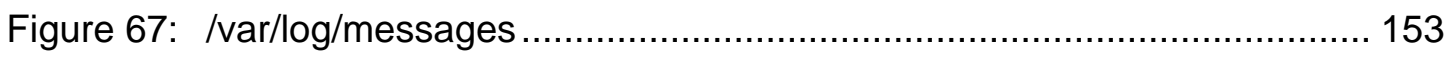

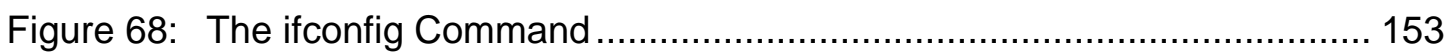

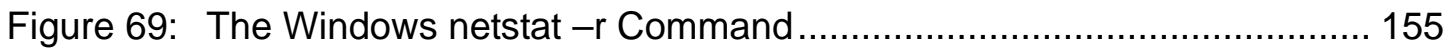

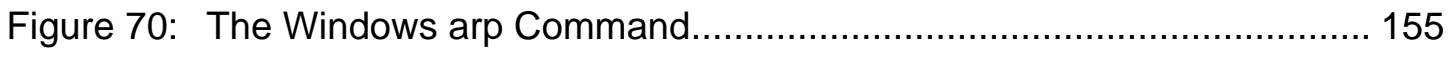

Figure 71: The Linux netstat -rn Command ................................................ 156

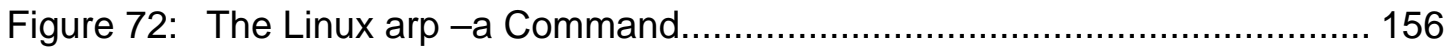

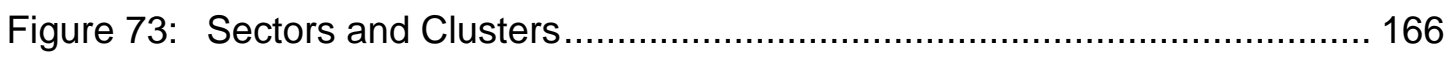

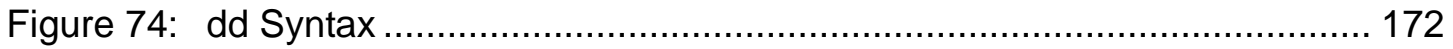

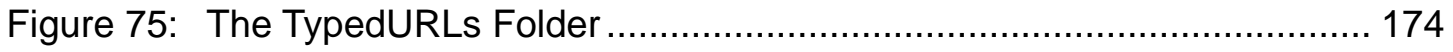

Figure 76: Sample Temp Folder Contents................................................. 176

Figure 77: The IE Default Location for Bookmarks ........................................ 179

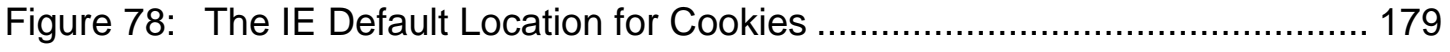

Figure 79: The IE Default Location for URL History ....................................... 180

Figure 80: The IE Default Location for Web Cache ......................................... 180

Figure 81: The Default Location for Outlook Email Files................................ 187 


\section{List of Tables}

Table 1: Default Cluster Sizes for Volumes with Windows XP Professional File

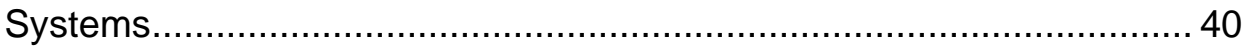

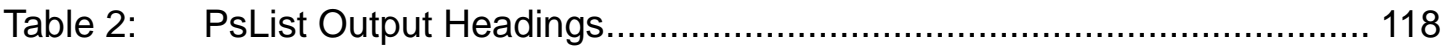

Table 3: $\quad$ w Command Output Fields ..................................................... 120

Table 4: $\quad$ A Subset of ps Options ............................................................... 120

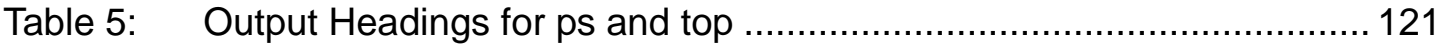

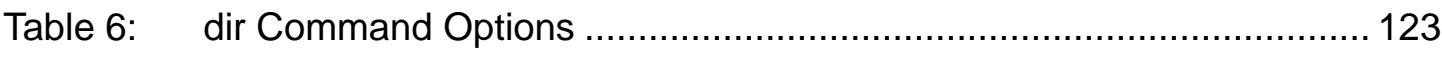

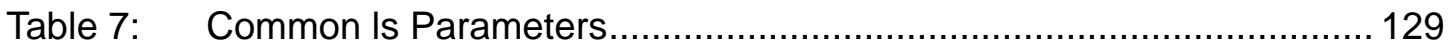




\section{Preface}

This handbook targets a critical training gap in the fields of information security, computer forensics, and incident response. In today's networked world, it is essential for system and network administrators to understand the fundamental areas and the major issues in computer forensics. Knowledgeable first responders apply good forensic practices to routine administrative procedures and alert verification, and know how routine actions can adversely affect the forensic value of data. This awareness will greatly enhance system and network administrators' effectiveness when responding to security alerts and other routine matters. This capability is a crucial and an often overlooked element of defense-in-depth strategies for protecting the availability, integrity, and survivability of IT and network infrastructures. For instance, the step of collecting data from a live system is often skipped because of time constraints, lack of preparation, and the common practice of returning the corrupted live system to its original state by either a fresh software installation or a system reboot.

This handbook is designed to familiarize experienced system and network computer professionals with the fundamental elements of computer forensics and build on their existing technical skill set. The experienced security professional will encounter little in the way of new technical information contained in this material, but will discover new approaches and methods. The primary objective is to educate and motivate system and network administrators to approach both routine and unusual events in a safe forensic manner. When they understand the importance of their role as a first responder, the safety and effectiveness of an organization's use of computer forensics will be greatly enhanced. The authors would like to thank Bill Spernow of Experian for taking the time to review this handbook and share useful insights about the handbook’s intended audience. 


\section{Abstract}

This handbook is for technical staff members charged with administering and securing information systems and networks. It targets a critical training gap in the fields of information security, computer forensics, and incident response: performing basic forensic data collection. The first module describes cyber laws and their impact on incident response. The second module builds understanding of file systems and outlines a best practice methodology for creating a trusted first responder tool kit for investigating potential incidents. The third module reviews some best practices, techniques, and tools for collecting volatile data from live Windows and Linux systems. It also explains the importance of collecting volatile data before it is lost or changed. The fourth module reviews techniques for capturing persistent data in a forensically sound manner and describes the location of common persistent data types. Each module ends with a summary and a set of review questions to help clarify understanding.

This handbook was developed as part of a larger project. The incorporated slides are from the five day hands-on course Forensics Guide to Incident Response for Technical Staff developed at the SEI. The focus is on providing system and network administrators with methodologies, tools, and procedures for applying fundamental computer forensics when collecting data on both a live and a powered off machine. A live machine is a machine that is currently running and could be connected to the network. The target audience includes system and network administrators, law enforcement, and any information security practitioners who may find themselves in the role of first responder. The handbook should help the target audience to

- understand the essential laws that govern their actions

- understand key data types residing on live machines

- evaluate and create a trusted set of tools for the collection of data

- collect, preserve, and protect data from live and powered off machines

- learn methodologies for collecting information that are forensically sound (i.e., able to withstand the scrutiny of the courts) 


\section{Module 1: Cyber Law}

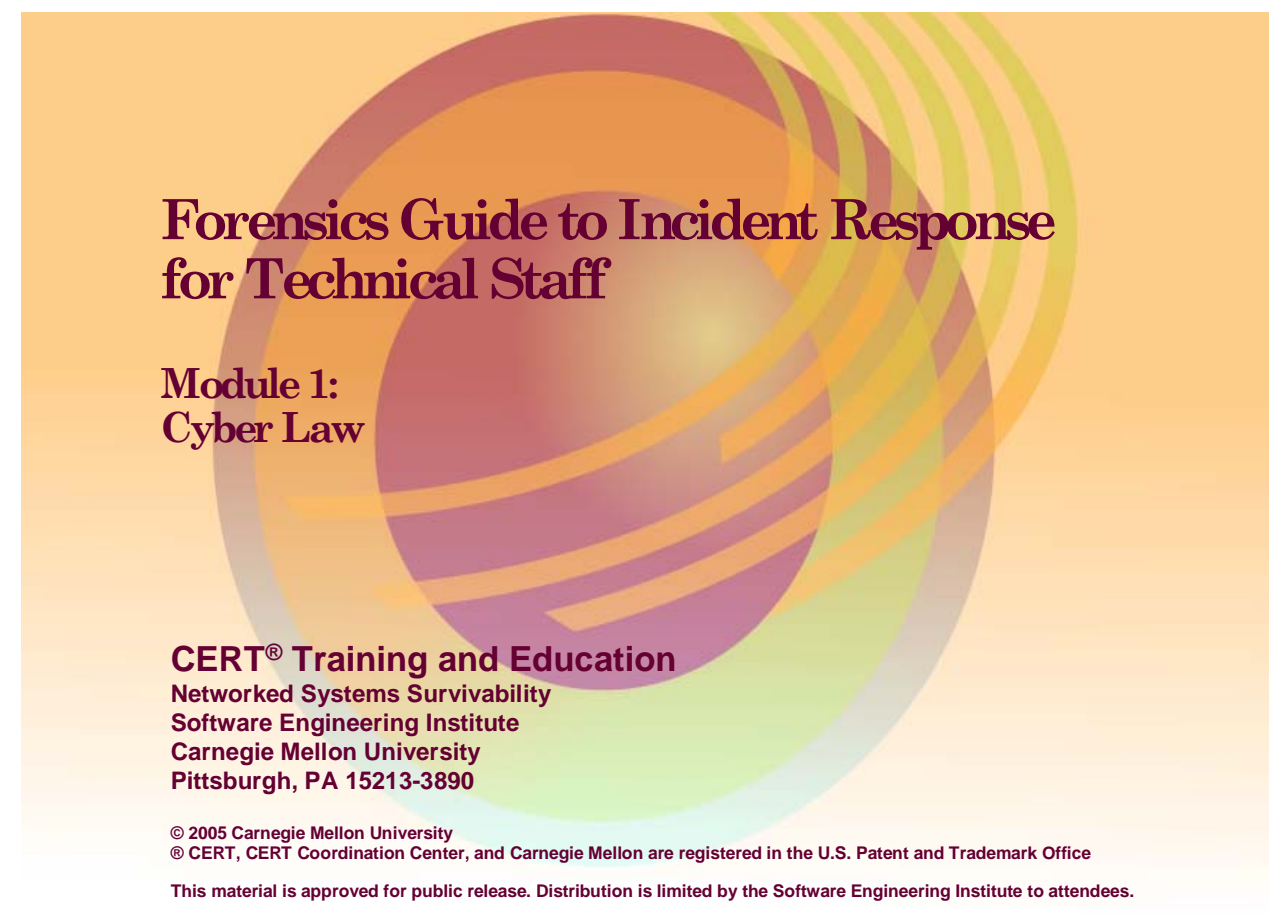

Module 1: Cyber Law discusses aspects of federal laws that affect computer security professionals. The objective is to motivate security professionals to frame and consider their technical actions in the context of existing legal governance. Throughout this module several complex legal issues are reviewed briefly. While many of these issues could occupy an entire course, our goal is to expose the reader to topical areas of cyber law and raise awareness of how these laws could potentially impact their actions.

Additionally, many scenarios are presented in a technical setting that demonstrates the impact of cyber laws. Scenarios are meant to help the reader understand the potential application and implications of cyber laws, and not as a definitive interpretation of the law. Every effort has been made to represent the laws accurately; however, this document is not an authoritative legal guide. 


\section{Objectives}

- Understand the role of a system administrator as a first responder to a security incident

- Understand the fundamentals of cyber laws:

1. The Wiretap Act

2. Pen/Trap and Trace Statute

3. Stored Electronic Communication Act

- Understand the impact of cyber laws on incident response

- Understand the fundamental areas of legal scrutiny that confront first responders to a security incident

(C) 2005 Carnegie Mellon University

\subsection{Module 1 Objectives}

The above slide lists the four primary objectives for Module 1, "Cyber Law.” 


\section{Forensics and Computer Forensics}

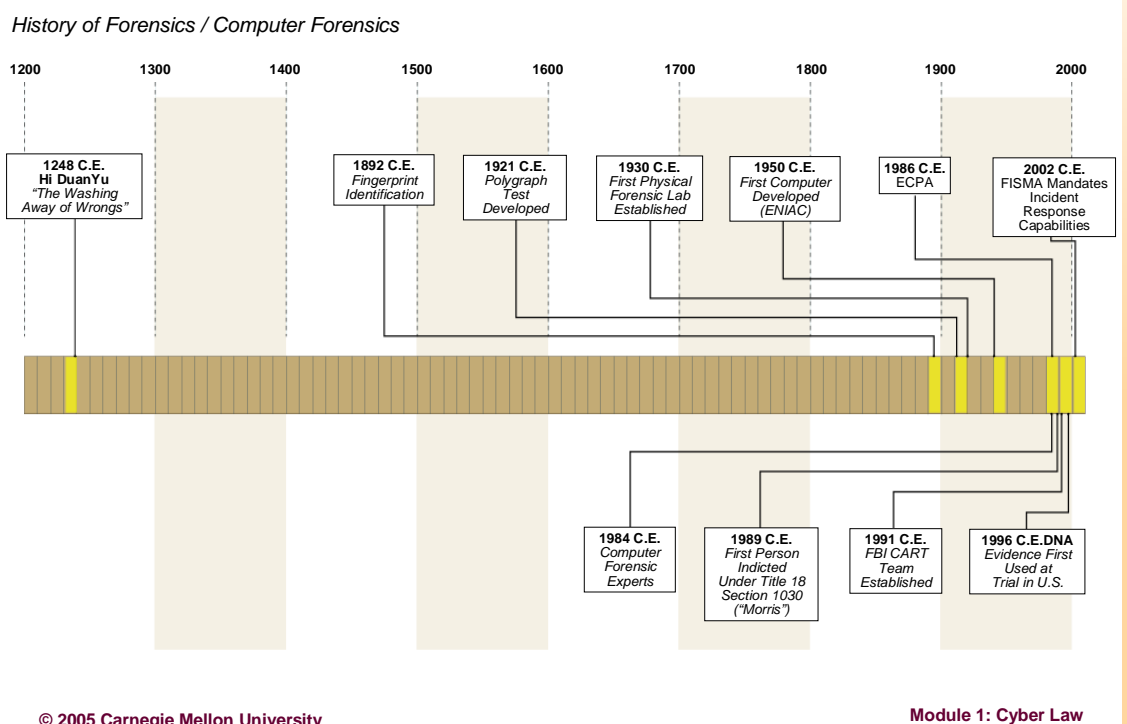

\subsection{Forensics}

Forensics is the process of using scientific knowledge in the collection, analysis, and presentation of evidence to the courts. The word forensics means "to bring to the court." Forensics deals primarily with the recovery and analysis of latent evidence. Latent evidence can take many forms, from fingerprints left on a window to DNA evidence recovered from blood stains. Forensics takes scientific knowledge and methods and combines them with standards of law for the purpose of recovering and presenting at court evidence related to a crime.

The field of forensics has developed over many centuries. The earliest record of forensics dates back to 1248 C.E., when a Chinese physician named Hi Duan Yu wrote The Washing Away of Wrongs. Yu presented the anatomical and medical knowledge of the time as it related to law, such as the difference between drowning, strangulation, and death by natural causes. ${ }^{1}$ Fingerprint identification was not used and understood until 1892. The first forensic lab was established in the United States in 1930. DNA evidence was first used as evidence at a trial in $1996 .{ }^{2}$

Although forensics has developed into a well-documented and disciplined field of study with many levels of certifications, it is always changing. As scientific knowledge and practices expand, so too will the processes of forensics. Nevertheless, forensics will always lag behind

\footnotetext{
$1 \quad$ http://www.mdpd.com/astmhtm.html

2 http://www.crimtrac.gov.au/dnahistory.htm
} 
the advances of science in some way. An example is DNA evidence-DNA typing was understood and utilized in the 1980s in New Zealand, but did not appear as evidence in the U.S. courts until 1996.

Considering that modern forensics has had almost six decades to develop in the U.S. courts and 800 years as a discipline, the specialized field of computer forensics, in comparison, is still in its infancy. It should come as no surprise, then, that there is very little standardization and consistency across the courts and industry in the area of computer forensics, as a result it is not yet recognized as a formal "scientific" discipline.

\subsubsection{Computer Forensics}

Computer forensics can be defined as the collection and analysis of data from computer systems, networks, communication streams (wireless) and storage media in a manner that is admissible in a court of law. It is a merger of the disciplines of computer science and the law. This definition applies to the collection of information in real time, as well as the examination of latent data.

Here is what others have to say about the definition of computer forensics:

"Computer forensics" can thus not afford solely to concern itself with procedures and methods of handling computers, the hardware from which they are made up and the files they contain. The ultimate aim of forensic investigation is use in legal proceedings [Mandia 01].

The objective in computer forensics is quite straightforward. It is to recover, analyze and present computer based material in such a way that it is useable as evidence in a court of law [Mandia 01].

The key phrase here is "useable as evidence in a court of law." It is essential that none of the equipment or procedures used during the examination of the computer obviate this single requirement. ${ }^{3}$

At one time, computer forensics dealt exclusively with persistent data-data that is stored on a local hard drive or other medium and is preserved when the computer is powered off-and was the sole domain of law enforcement. In this age of interconnected networks, however, the examination and collection of volatile data is becoming more important. Volatile data is any data that is stored in memory, or in transit, that will be lost when the computer loses power or is powered off. Volatile data resides in registries, cache, and random access memory (RAM). Since the nature of volatile data is effervescent, collection of this information will likely need to occur in real or near-real time.

3 http://www.dibsusa.com/methodology/methodology.html 
Also, with the rapid expansion of the Internet and the introduction of recent legislation such as HIPAA, ${ }^{4}$ Sarbanes-Oxley, ${ }^{5}$ California Act $1798,{ }^{6}$ and others, all of which hold businesses civilly and, in some cases, criminally liable for breaches in the security or integrity of computer networks and systems, companies are not only trying to protect themselves from Internet-related threats but are also dealing with compliance and liability concerns related to these laws. Several current writings point to the fact that companies are allocating more of their IT budgets towards security. ${ }^{7}$ In fact, International Data Corporation (IDC) has reported that the market size for intrusion-detection and vulnerability-assessment software will reach 1.45 billion dollars in 2006. These are relatively well-known trends in the IT community. These factors and others have increased the deployment of network security devices such as intrusion detection systems (IDS), firewalls, proxies, etc., which all report on the security status of networks to some degree in real time or near-real time. So much of security is focused on locking down and monitoring systems that an often overlooked area is how information and evidence related to the alerts are technically handled.

Almost always, when a security breach or monitoring alert occurs, some sort of verification needs to happen. No matter what type of alert is generated, verification is required to determine whether the alert is a false positive or is in fact an indication of some breach, attempted breach, or incident. This verification, often a form of routine administrative task, is almost always conducted on a live machine, and deals with volatile data. System administrators and security personnel must possess a basic understanding of how routine administrative tasks can affect both the forensic process (the potential admissibility at court) and the subsequent ability to recover data that can potentially be critical to the identification and analysis of a security incident.

Contrary to the impression given by popular television shows, there are not nearly enough forensic investigators available to respond to every crime scene to collect, process, and analyze evidence. As a result, many law enforcement officers are being trained to process and collect evidence (fingerprints, fibers, etc.) from crime scenes in a forensically sound manner. A qualified investigator then analyzes the evidence and makes conclusions at a later time. At trial, or before the courts, the collector authenticates the collected evidence and the expert testifies to its significance. This division of labor is also needed in the discipline of computer forensics. Like law enforcement officers, system administrators are often the "first responders” at potential "crime scenes.” It is critical that they possess the technical skills and ability to preserve critical information related to a suspected security incident in a

4 Health Insurance Portability and Accountability Act of 1996 (see http://www.hhs.gov/ocr/hipaa/)

5 Sarbanes-Oxley Act of 2002 (http://frwebgate.access.gpo.gov/cgi-

bin/getdoc.cgi?dbname=107_cong_public_laws\&docid=f:publ204.107.pdf).

6 Information Practices Act of 1977 (http://www.privacy.ca.gov/code/ipa.htm).

7 http://www.vnunet.com/news/1150059,

http://itmanagement.earthweb.com/it_res/print.php/3085471,

http://www.csoonline.com/csoresearch/report50.html

CMU/SEI-2005-HB-001 
forensically sound manner and that they be aware of the legal issues that govern their actions. This skill set is not easily acquired, and is the subject of many courses of study.

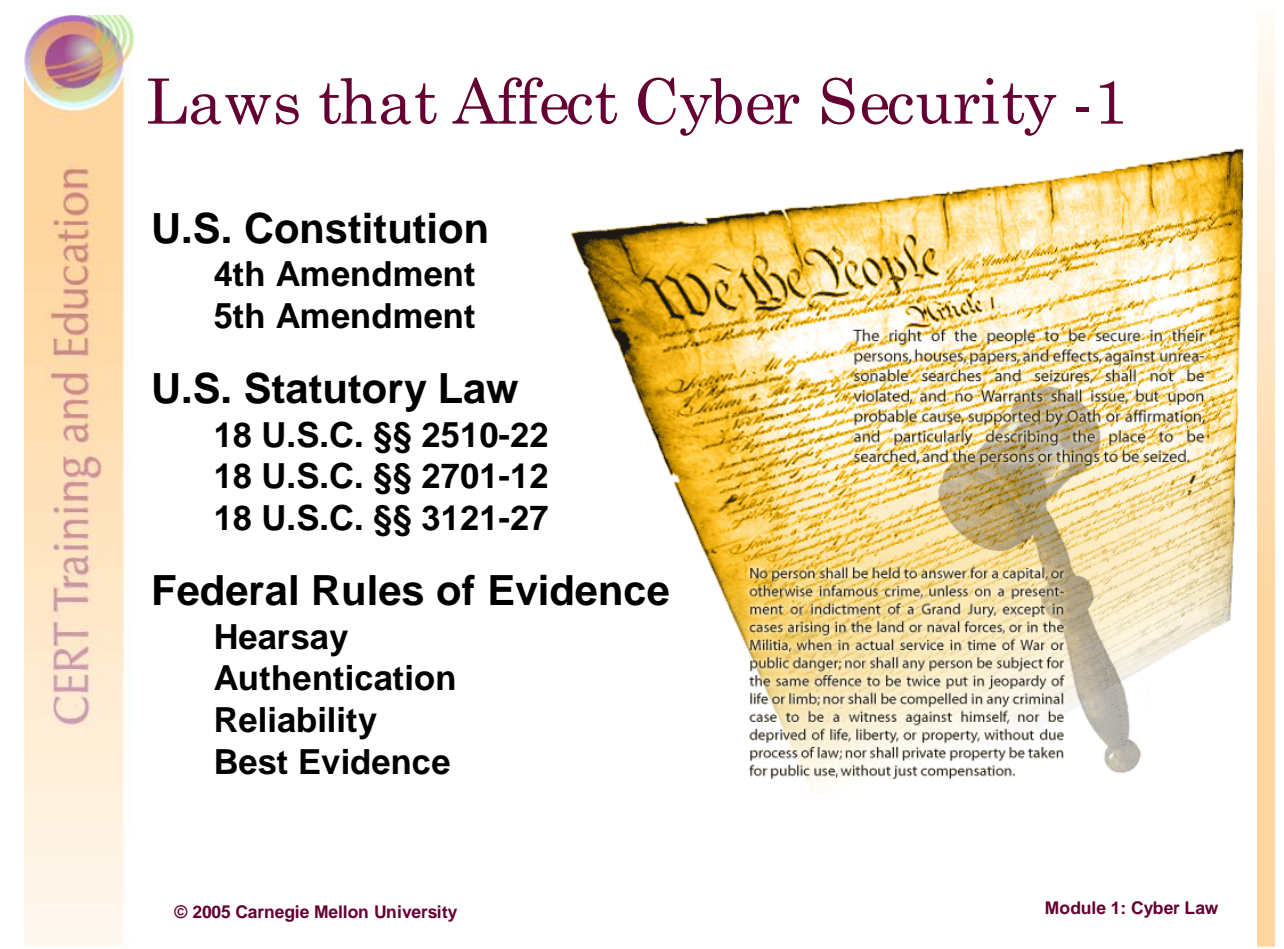

\subsection{Laws that Affect Cyber Security}

Computer forensics is a relatively new discipline to the courts and many of the existing laws used to prosecute computer-related crimes, legal precedents, and practices related to computer forensics are in a state of flux. Almost daily, new court rulings are handed down that affect how computer forensics is applied. The best source of information in this area is the United States Department of Justice's Cyber Crime Web Site. ${ }^{8}$ Here all the recent court cases can be reviewed, as well as numerous guides on the introduction of computer evidence in court and what standards apply.

One recent court case, United States Court of Appeals for the First Circuit, No. 03-1383 UNITED STATES OF AMERICA, Appellant, v. BRADFORD C. COUNCILMAN, Defendant, Appellee, has a couple of interesting aspects: (1) it is a landmark case in that it could fundamentally change the way emails are dealt with and (2) the courts acknowledge that they are "wrestling with the antiquated nature of some of the laws." Circuit Judge Juan R. Torruella wrote this in his summary:

Moreover, at this juncture, much of the protection [of the Wiretap Act] may have been eviscerated by the realities of modern technology. We observe, as most courts have, that the language may be out of step with the technological realities

8 http://www.cybercrime.gov 
of computer crimes. This particular appeal reflects how the Courts are wrestling with the antiquated nature of some of the laws governing electronic communications. $^{9}$

The appeal was over whether email should be considered stored electronic communication or whether it should considered transmitted electronic communication. The outcome of this case was that the court did not believe that emails are protected by the Wiretap Act under the Store Communication Section when they are in temporary file storage at various locations along their delivery route. Although the court ruled in favor of the defendant, an interesting dissenting opinion was presented. Essentially, the dissenting judge uses sections of an RFC on emails ${ }^{10}$ and other very technical information and expresses two things: (1) that the current law was never intended to be applied to this type of technology and (2) that the "intent" of the law only protects email while it is being transmitted from sender to receiver.

Again, keep in mind that this module is meant not to be a course in legal studies but rather to serve as an introductory exposure to major legal governance issues that affect system administrators and network security personnel. To use a training analogy from law enforcement, police officers are not lawyers, nor will their training ever make them so; however, police officers receive training in legal areas that authorize their actions and govern their responses. For example, police officers all have a fundamental understanding of the restrictions of the $4^{\text {th }}$ and $5^{\text {th }}$ Amendments of the U.S. Constitution and the several exceptions to them. This is because these two amendments have significant impact on how law enforcement officers conduct themselves. This same model needs to be applied to system administrators and network security personnel. This is not to say that they should try to function as law enforcement, but rather that they should be aware of and understand the fundamental legal issues governing their actions.

9 http://www.ca1.uscourts.gov/cgi-bin/getopn.pl?OPINION=03-1383.01A

10 RFC 821, by Jonathan B. Postel (see http://www.faqs.org/rfcs/rfc821.html). 


\title{
Laws that Affect Cyber Security -2
}

\author{
Authority to Monitor \& Collect Admissibility of Collection \\ U.S. Constitution \\ 4th Amendment \\ U.S. Statutory Law \\ (Stored Data) \\ 18 U.S.C. §§ 2701-12

\section{U.S. Constitution} \\ 5th Amendment \\ Federal Rules of Evidence \\ Hearsay \\ Authentication \\ Reliability Best Evidence
}

(Real Time)

18 U.S.C. §§ 2510-22

18 U.S.C. §§ 3121-27

Three areas of law related to computer security will be examined:

1. U.S. Constitution

- $\quad 4^{\text {th }}$ Amendment "Protection against unreasonable search and seizure"

- $\quad 5^{\text {th }}$ Amendment "Protection against self incrimination"

2. U.S. Statutory Law

- 18 U.S.C. 2510-22 (The Wiretap Act )

- 18 U.S.C. 3121-27 (The Pen Registers and Trap and Trace Devices Statute)

- 18 U.S.C. 2701-12 (The Stored Wired and Electronic Communication Act)

3. Federal Rules of Evidence

- Hearsay

- Authentication

- Reliability

- $\quad$ Best Evidence

This is not a comprehensive review of these legal areas but rather a summary review of key aspects that will likely affect the actions of system and network administrators.

In the United States of America, there are two primary areas of legal governance affecting cyber security actions related to the collection of network data: (1) authority to monitor and collect the data and (2) the admissibility of the collection methods. Of the three areas above, the U.S. Constitutional and U.S. Statutory Laws primarily govern the collection process, while the Federal Rules of Evidence deal primarily with admissibility. 


\section{Laws that Affect Monitoring and Collection -1}

\section{U.S. Constitution (only limits government action)}

\section{4th Amendment:}

Protection against unreasonable search and seizure Katz Standard:

1. Subjective expectation of privacy

2. Viewed by society as reasonable

\section{5th Amendment:}

Protection against self incrimination Encryption and private keys

\subsection{Legal Governance Related to Monitoring and Collection}

A key point to remember when considering these amendments is that they were written some two hundred years ago and are now being applied to a technology that could not be imagined by the original authors.

\subsubsection{Constitutional Issues}

\subsubsection{The $4^{\text {th }}$ Amendment}

The $4^{\text {th }}$ Amendment to the United States Constitution is as follows:

The right of the people to be secure in their persons, houses, papers, and effects, against unreasonable searches and seizures, shall not be violated, and no Warrants shall issue, but upon probable cause, supported by Oath or affirmation, and particularly describing the place to be searched, and the persons or things to be seized.

The $4^{\text {th }}$ Amendment protects individuals from unreasonable searches and seizures conducted by agents of the government. It does not extend protection from searches conducted by private parties who are not acting as agents of the government. The question is whether or not your organization can be considered as "government", or under the direction of a government agent, under the $4^{\text {th }}$ Amendment. There isn't always a bright line answer to that question (in law, a bright line is a rule that clearly and unambiguously delineates what is so 
and what is not). If in doubt, organizations should always seek the advice of legal counsel in determining the answer.

In general, the principles set forth by the $4^{\text {th }}$ Amendment provide for individuals to enjoy a "reasonable expectation of privacy.” In determining what is a reasonable expectation of privacy, the U.S. courts rely in part on the Katz test (Katz v. United States, 389 U.S. 347, 362 (1967). The Katz test outlines two questions: (1) Does the individual's conduct demonstrate a (subjective) expectation of privacy? and (2) Would society be prepared to recognize this as reasonable? These questions allow for a range of interpretations and are the subject of a significant amount of case law. However, they are the fundamental questions asked by the $4^{\text {th }}$ Amendment. To explore a more detailed analysis of the issues surrounding the $4^{\text {th }}$ Amendment, the following site is a good place to start: http://caselaw.lp.findlaw.com/data/constitution/amendment04/.

What system administrators and network security personnel need to understand is that individuals may enjoy a certain amount of privacy in the electronic world (emails, stored files, etc.). Some other key points are (1) the $4^{\text {th }}$ Amendment does not apply to or restrict the conduct of private individuals, only that of the government; (2) the level of expected privacy is substantially less outside of the home, although not eliminated; and (3) protections (privacy) enjoyed from the $4^{\text {th }}$ Amendment are dissolved when the item protected by this amendment is lost or otherwise ends up no longer in the custody of the owner. Also, if information or evidence is collected in a way that is determined to have violated the $4^{\text {th }}$ Amendment, that information/evidence and all information/evidence subsequently obtained or discovered as a result of the original information/evidence will be suppressed, i.e., deemed inadmissible by the courts. This is known as the "fruit of the poisonous tree doctrine."

The $4^{\text {th }}$ Amendment has been applied to information technology in a number of cases. The following is an excerpt from a U.S. Department of Justice Manual: ${ }^{11}$

When confronted with this issue, courts have analogized electronic storage devices to closed containers, and have reasoned that accessing the information stored within an electronic storage device is akin to opening a closed container. Because individuals generally retain a reasonable expectation of privacy in the contents of closed containers, see United States v. Ross, 456 U.S. 798, 822-23 (1982), they also generally retain a reasonable expectation of privacy in data held within electronic storage devices. Accordingly, accessing information stored in a computer ordinarily will implicate the owner's reasonable expectation of privacy in the information. See United States v. Barth, 26 F. Supp. 2d 929, 93637 (W.D. Tex. 1998) (finding reasonable expectation of privacy in files stored on hard drive of personal computer); United States v. Reyes, 922 F. Supp. 818, 83233 (S.D.N.Y. 1996) (finding reasonable expectation of privacy in data stored in a pager); United States v. Lynch, 908 F. Supp. 284, 287 (D.V.I. 1995) (same);

11 http://www.cybercrime.gov/s\&smanual2002.htm 
United States v. Chan, 830 F. Supp. 531, 535 (N.D. Cal. 1993) (same); United States v. Blas, 1990 WL 265179, at *21 (E.D. Wis. Dec. 4, 1990) ("[A]n individual has the same expectation of privacy in a pager, computer, or other electronic data storage and retrieval device as in a closed container.”).

\subsubsection{The $5^{\text {th }}$ Amendment}

The 5th Amendment provides that

No person shall be compelled in any criminal case to be a witness against himself.

The core issue related to computer forensics is that under current jurisprudence the $5^{\text {th }}$ Amendment's protections against self incrimination extend to cryptographic keys. "Under the Fifth Amendment, an individual cannot be compelled to testify to his or her memorized key" [CDT 04]. The word "memorized" is an important qualifier, however, meaning that the key (passphrase) was never written down. Remember, the $5^{\text {th }}$ Amendment only protects an individual from being compelled to provide incriminating testimony. Its protections do not extend to existing written and/or documentary evidence. This affects system or network administrators because if encrypted files are found during a collection or examination of a system or network, little can be done to force an individual to decrypt them.

For more details (in significant depth), visit the following Web sites:

- http://www.cdt.org/crypto/legis_105/mccain_kerrey/const_impact.html

- http://law.richmond.edu/jolt/v2i1/sergienko.html\#h1 


\title{
Laws that Affect Monitoring and Collection -2
}

\section{U.S. Statutory Law}

\author{
18 U.S.C. §§ 2510-22 Wiretap Act \\ 18 U.S.C. §§ 3121-27 Pen/Trap and Trace \\ 18 U.S.C. \&\& 2701-12 Stored Electronic Communication Act
}

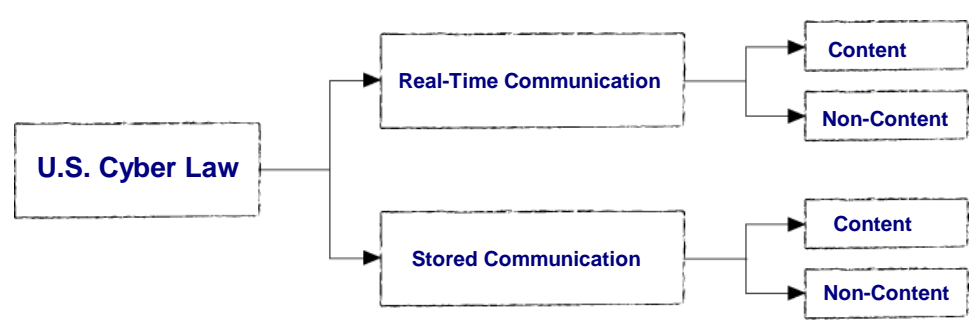

(C) 2005 Carnegie Mellon University

Module 1: Cyber Law

\subsubsection{U.S. Statutory Law}

There are three U.S. statutes that potentially impact the techniques used by system administrators and network security personnel during the collection of computer records and other digital evidence in response to a security incident:

- 18 U.S.C. $\S \S 2510-22 \quad$ Wire and Electronic Communications Interception and Interception of Oral Communications

- 18 U.S.C. $\S \S 3121-27 \quad$ Pen Registers and Trap and Trace Devices

- 18 U.S.C. $\S \S 2701-12 \quad$ Stored Wire and Electronic Communications and Transactional Records Access

This section will highlight the intent and purpose of these statutes, as well as provide technical scenarios illustrating the exceptions to these statutes as they affect the actions taken by system administrators and network security personnel. Absent these exceptions, violations of these statutes could constitute a federal felony punishable by a fine and/or prison.

Of these three statutes, two deal with real-time electronic communications (18 U.S.C. §§ 2510-22 and 18 U.S.C. $\S \S 3121-27$ ) and the other (18 U.S.C. §§ 2701-12) deals with stored electronic communications. 


\section{Laws that Affect Monitoring and Collection -3}

\section{Real-Time Communication: Content vs. Non-Content}

18 U.S.C. §§ 3121-27 Pen/Trap and Trace

18 U.S.C. $\S \S 2510-22$ Wiretap Act

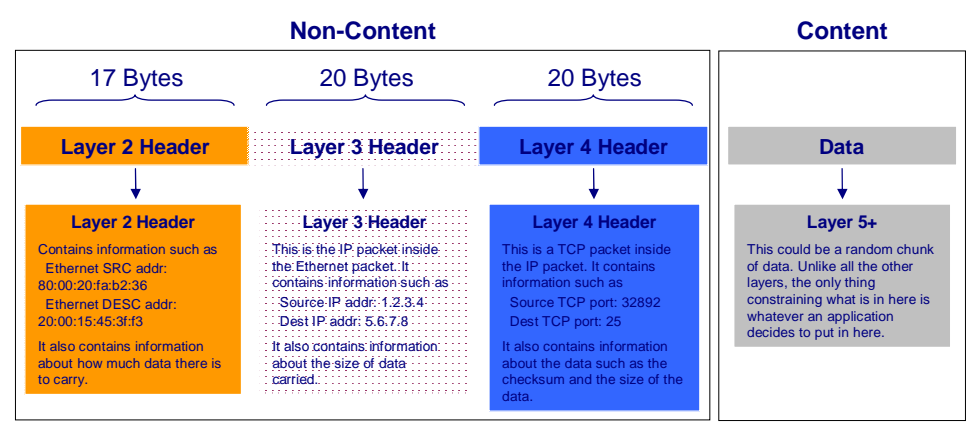

๑ 2005 Carnegie Mellon University

Module 1: Cyber Law

The Wiretap Act and Pen/Trap and Trace both deal with real-time communication, but they focus on very different aspects of that communication. It is important to note that both of these statutes are not clearly written to apply to the flow of data over the Internet, as they were born in an era when telecommunications was the primary medium of communication. They have been recently modified to better clarify their governance related to electronic communications, but there is little technical language embedded in the actual statutes themselves.

Below is a technical review of the Open System Interconnection (OSI) Model. Within the existing language of the above-mentioned statues the intent is that the Pen/Trap and Trace deals with Layer 2, 3, and 4 information and the Wiretap Act deals with Layer 5, 6, and 7 information.

\section{Layer 1: Physical}

This is the physical wire that carries the electronic pulses.

Layer 2: Data Link (17 bytes)

Contains information such as the media access control (MAC) addresses of the source sending the packet and the next hop or destination.

Layer 3: Network (20 bytes)

Contains information such as the source and destination IP addresses.

Layer 4: Transport (20 bytes)

Contains information such as source and destination ports. 


\section{Layer 5+: Session/Presentation/Application}

This contains the data that the application requires (for example, this would be the data portion of an email message).

Often the Department of Defense (DoD) Model or Internet Protocol Suite and the Open System Interconnection (OSI) Model are used to describe the same process. Below is a mapping of the two models.

\section{DoD Model}
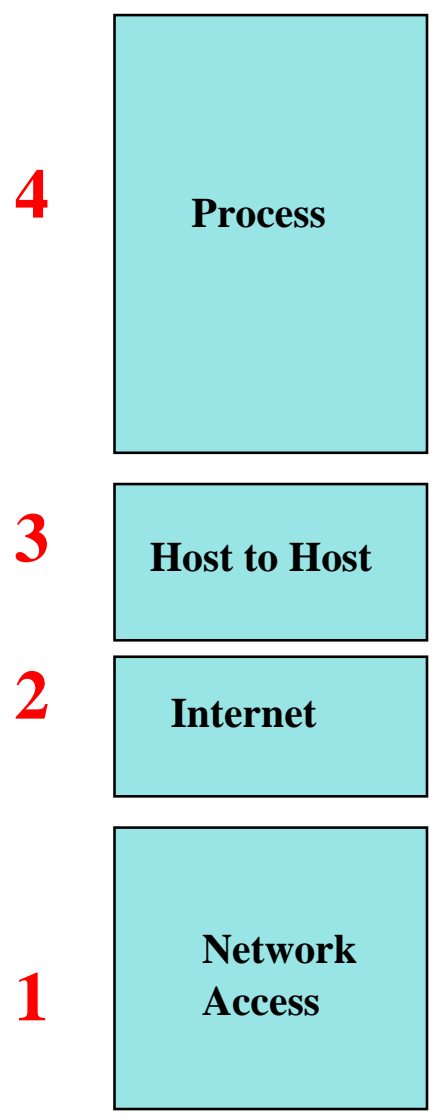

\section{OSI Model}
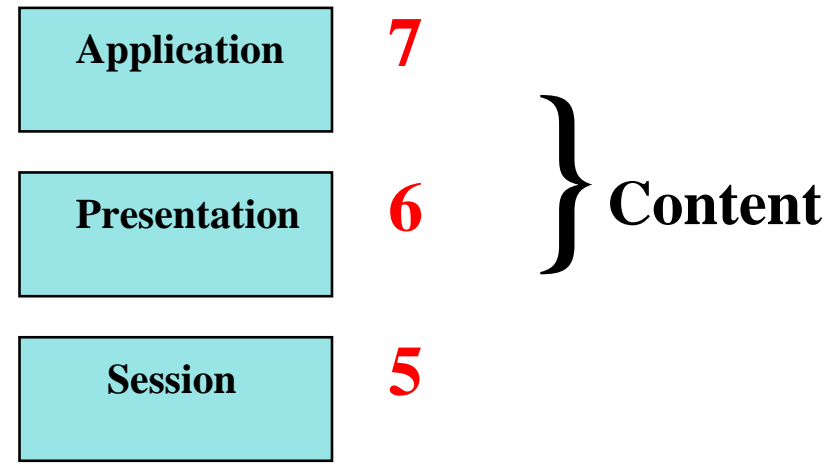

5
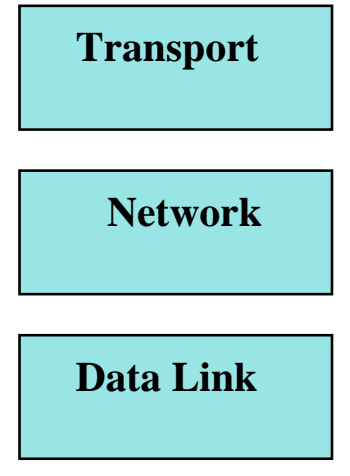

2

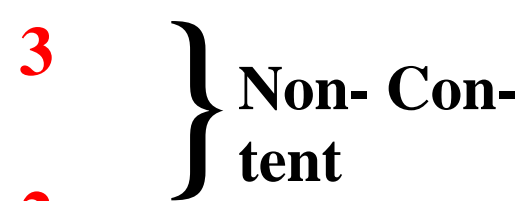

Figure 1: Mapping of DoD and OSI Models

It is important to understand the fundamental difference between what the laws consider “content” and "non-content." Although there is no bright line, from a technical perspective the division occurs between Layer 4 (Layers 4 and below deal with routing, addressing, and signaling information) and Layer 5 (Layers 5 and above deal with the Data or Content). 


\section{Laws that Affect Monitoring and Collection -4}

\section{Wiretap Act}

18 U.S.C. $\S \S 2510-22$

Prohibited Acts:

intentionally intercepts, endeavors to 18 USC 2511(1)(a) intercept, or procures any other person to intercept or endeavor to intercept, any wire, oral, or electronic communication

\section{Exceptions:}
1) Provider Exception
2) Consent
3) Trespasser Exception

\subsubsection{Wiretap Act/Electronic Communications Privacy Act}

Generally speaking, the Wiretap Act, 18 U.S.C. $\S \S 2510-22$, prohibits the interception of realtime electronic data communications (specifically the content), unless one of the statutory exceptions applies. There are several exceptions, but only two exceptions that potentially affect incident response activities will be considered in this documentation.

This law governs the usage of tools like sniffers (TCPDump, Ethereal, Etherpeek, etc.), which are used to intercept and examine the content (i.e., Layer 5+) of electronic data communication and any other device that looks at the content (Layer 7 firewalls, URL scanners, etc.). This law does not restrict the interception and examination of signaling information (information from the network and transport layers). Those actions are restricted by the Pen/Trap and Trace Statute, which is discussed later.

The Wiretap Act applies to anyone who

intentionally intercepts, endeavors to intercept, or procures any other person to intercept or endeavor to intercept, any wire, oral, or electronic communication.

There are several exceptions enumerated in the statute. Of these, three have the greatest impact on the actions of system administrators and network security personnel.

1) Provider Exception, 18 U.S.C. § 2511(2)(a)(i)

It shall not be unlawful under this chapter for an operator of a switchboard, or an officer, employee, or agent of a provider of wire or electronic communication service, whose facilities are used in the transmission of a wire or electronic 
communication, to intercept, disclose, or use that communication in the normal course of his employment while engaged in any activity which is a necessary incident to the rendition of his service or to the protection of the rights or property of the provider of that service, except that a provider of wire communication service to the public shall not utilize service observing or random monitoring except for mechanical or service quality control checks.

This allows for system administrators and network security personnel to conduct limited monitoring for the purpose of protecting the "rights or property" of the provider. It is permissible to monitor the content of electronic communications under this exception when there is a clear nexus between the threat and what is monitored.

Here is a scenario that demonstrates an example of a clear nexus between a threat to the "rights or property" of a provider and what is being monitored:

In support of Web services that are critical to his operations, a system administrator is using a URL scanner. He is examining the "contents" of the Web requests, not just the connection-related information (Layers 3 and 4). A clear nexus exists between this monitoring of "content" and the protection of the Web server."

2) Consent, 18 U.S.C. § 2511(2)(c)

...where such person is a party to the communication or one of the parties to the communication has given prior consent to such interception...

Here is where bannering the network and written and signed acceptable use policies are critical. If you have a correctly worded banner and an acceptable use policy that informs users of monitoring activities and how the information gathered from monitoring will be used, in a vast majority of cases the consent burden will be satisfied.

An example of this would be when a company institutes a policy that forbids nonbusiness-related Web surfing and states that monitoring of Web surfing will be conducted to ensure compliance. Here clear consent is required by the users of the system. Given this consent to a written or bannered policy, the company can intercept and monitor Web surfing activities to ensure compliance with the policy. ${ }^{*}$

Consent should never be taken as a general permission for system administrators to conduct ad hoc, random monitoring activities. Rather, monitoring activities related to consent should be part of a well-documented process that is explicitly enumerated in the obtained consent.

* This example is not intended to be legally authoritative but to frame a legal concept in terms that a system administrator will understand. 
3) Trespasser Exception, 18 U.S.C. § 2510(21)

... a person who accesses a protected computer without authorization and thus has no reasonable expectation of privacy in any communication transmitted to, through, or from the protected computer...

This is a very broad exception because of the definition of a "protected computer." Basically almost every public provider of electronic communication falls under this definition. Consultation with counsel should be sought to make an authoritative interpretation. The definition of "protected computer" is found in 18 U.S.C. 1030(21): ${ }^{12}$

The term "protected computer" means a computer

(A) exclusively for the use of a financial institution or the United States

Government, or, in the case of a computer not exclusively for such use, used by or for a financial institution or the United States Government and the conduct constituting the offense affects that use by or for the financial institution or the Government; or

(B) which is used in interstate or foreign commerce or communication, including a computer located outside the United States that is used in a manner that affects interstate or foreign commerce or communication of the United States...

An example scenario of this exception would be as follows:

A system administrator is on duty when she receives a remote syslog message from a Linux system that is running a SQL database indicating that the ftp service was started. Upon further inspection of the syslog files, she also discovers that moments before the ftp alert, a "root” login occurred. This clearly presents a security threat to that system. There is an unauthorized root login and potentially an ftp session in progress. At this point, in an effort to protect the network, the system administrator runs a sniffer to begin monitoring and collecting the entire session originating from that system.*

It is also a best practice to post banners on network devices. This practice was more important prior to the U.S. Patriot Act, when it was unclear whether a "trespasser" enjoyed protection under the Wiretap Act. With the Patriot Act, there is an exception that specifically deals with trespassers. However, posting banners on network devices indicating that monitoring is occurring and how that monitoring will be used is still a best practice.

12 Fraud and Related Activity in Connection with Computers (see http://www.cybercrime.gov/1030_new.html)

* This example is not intended to be legally authoritative but to frame a legal concept in terms that a system administrator will understand. 


\section{Laws that Affect Monitoring and Collection -5}

\section{Pen/Trap and Trace Act}

18 U.S.C. $\S \S 3121-27$

\section{Prohibited Acts:}

No person may install or use a pen register or 18 U.S.C. 3121(a) a trap and trace device without first obtaining a court order under section 3123 of this title or under the Foreign Intelligence Surveillance Act.

\section{Exception:}
1) Provider Exception
2) Verification of Service
3) Consent

๑ 2005 Carnegie Mellon University

Module 1: Cyber Law

\subsubsection{Pen Registers and Trap and Trace Devices}

Whereas the Wiretap Act focuses on the content of electronic communications, this statute, 18 U.S.C. $\S \S 3121-27$, prohibits the installation of a device that records or decodes dialing, routing, addressing, or signaling information for outgoing (Pen Registers) and incoming (Trap and Trace) wired or electronic communications, except for certain purposes.

Statutory definitions:

- $\quad$ Pen Register - 18 U.S.C.§ 3127(3)

The term "pen register" means a device or process which records or decodes dialing, routing, addressing, or signaling information transmitted by an instrument or facility from which a wire or electronic communication is transmitted, provided, however, that such information shall not include the contents of any communication, but such term does not include any device or process used by a provider or customer of a wire or electronic communication service for billing, or recording as an incident to billing, for communications services provided by such provider or any device or process used by a provider or customer of a wire communication service for cost accounting or other like purposes in the ordinary course of its business.

- $\quad$ Trap and Trace -18 U.S.C.§ 3127(4)

The term "trap and trace device" means a device or process which captures the incoming electronic or other impulses which identify the originating number or other dialing, routing, addressing, and signaling information reasonably likely to 
identify the source of a wire or electronic communication, provided, however, that such information shall not include the contents of any communication.

Within the definition one major point is clearly made: these devices (pen registers and trap and trace devices) deal with non-content information. This statute governs the use of tools that are used to record and collect signaling information (i.e., Layers 3 and 4), such as Netlog and Argus. Notice that there is an absence of intent-specific terms.

A prohibited act under this statute, 18 U.S.C. $\S 3121(a)$, specifies that

no person may install or use a pen register or a trap and trace device without first obtaining a court order...

There are three exceptions to this statute, 18 U.S.C. $\S \S \S 3121(b)(1),(2)$, and (3).

1) Provider Exception 18 U.S.C. § 3121(b)(1)

...relating to the operation, maintenance, and testing of a wire or electronic communication service or to the protection of the rights or property of such provider, or to the protection of users of that service from abuse of service or unlawful use of service...

The following is an example scenario for this exception:

A network administrator runs Argus to monitor and analyze netflow traffic. Although this is a collection of signaling information, it is permitted under the Provider Exception because it is related to the maintenance and protection of the provider.

2) Verification of Service 18 U.S.C. § 3121(b)(2)

...to record the fact that a wire or electronic communication was initiated or completed in order to protect such provider, another provider furnishing service toward the completion of the wire communication, or a user of that service, from fraudulent, unlawful or abusive use of service...

The following is an example scenario for this exception:

Companies that offer services on a per use basis maintain records of usage to ensure proper billing. For example, some cellular telephone companies offer text messaging on a per use basis. Records of the number and size of the messages sent to and from an account will likely be collected.*

* This example is not intended to be legally authoritative but to frame a legal concept in terms that a system administrator will understand. 


\section{3) Consent 18 U.S.C. $\S 3121(b)(3)$}

...where the consent of the user of that service has been obtained.

Again, here is where bannering the network and written and signed acceptable use policies are critical. If you have a correctly worded banner and an acceptable use policy that informs users of monitoring activities and how the information gathered from monitoring will be used, in most cases the consent burden will be satisfied. 


\section{Laws that Affect Monitoring and Collection -6}

\section{Stored Wire \& Electronic Communication Act}

18 U.S.C. \&\& 2701-12

\section{Content vs. Non-Content}

Focus: prohibited actions of a "public provider" in regards to accessing and voluntarily disclosing stored electronic communications

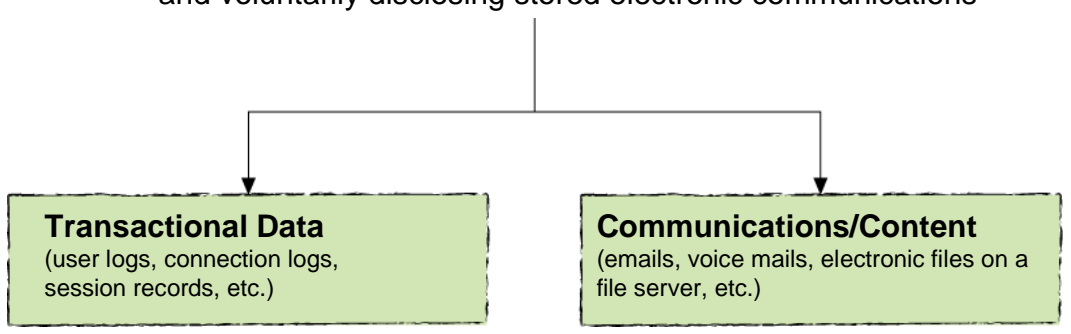

๑) 2005 Carnegie Mellon University

Module 1: Cyber Law

\subsubsection{Stored Wired and Electronic Communications Act}

The Stored Wired and Electronic Communication Act, 18 U.S.C. §§ 2701-12, deals with general protections for stored communications. There are several other statutes that may affect the level of protection, access, and disclosure of stored electronic communication, such as HIPAA and the Family Educational Rights and Privacy Act (FERPA). Consultation with legal counsel should be sought regarding whether your organization may be governed by these special types of privacy laws.

Like the Wiretap Act and the Pen/Trap and Trace Statute, the Stored Wired and Electronic Communication Act deals with content and non-content differently. 


\section{Laws that Affect Monitoring and Collection -7}

\section{Stored Wire \& Electronic Communication Act}

18 U.S.C. $\S \S 2701-12$

Prohibited Acts:

a person or entity providing an electronic communication service to the public shall not knowingly divulge to any person or entity the contents of a communication while in electronic storage by that service

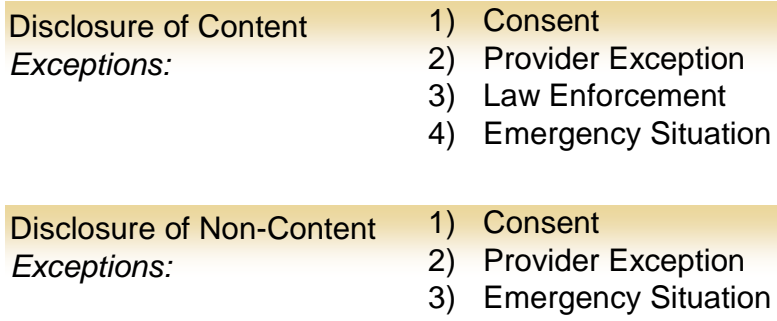

2) Provider Exception

3) Law Enforcement

4) Emergency Situation

Disclosure of Non-Content

1) Consent

Exceptions:

2) Provider Exception

3) Emergency Situation

() 2005 Carnegie Mellon University

Module 1: Cyber Law

Whereas the Wiretap and Pen/Trap and Trace statutes deal with real-time communications, this statute deals with and provides general protection from access to and disclosure of stored electronic communications. There are several sections to this statute, but for our purpose we will look only at the prohibited acts of public providers regarding access and voluntary disclosure of stored electronic communications. This section does not prohibit the actions of private providers who may voluntarily disclose content (stored voice or email), transactional data, and user information to government and non-government entities. Whether your organization is a public provider or private provider should be determined by counsel.

There are more prohibited acts under this statute than addressed here. This is a very complex statute that contains various restrictions. For the purpose at hand, only the following prohibited acts under the statute (18 U.S.C. § 2702) will be discussed:

(1) a person or entity providing an electronic communication service to the public shall not knowingly divulge to any person or entity the contents of a communication while in electronic storage by that service; and

(2) a person or entity providing remote computing service to the public shall not knowingly divulge to any person or entity the contents of any communication which is carried or maintained on that service-

(3) a provider of remote computing service or electronic communication service to the public shall not knowingly divulge a record or other information pertaining to a subscriber to or customer of such service (not including the 
contents of communications covered by paragraph (1) or (2)) to any

governmental entity.

This statute breaks down stored communication into three areas: (1) communications content (emails, voice mails, electronic files on a file server, etc.), (2) transactional data (user logs, connection logs, session records, etc.), and (3) subscriber information. This breakdown has more to do with the mandatory disclosure section of this statute than with issues that system administrators and network security personnel will deal with, but is nevertheless worth noting.

The exceptions to this statute are broken into two categories: content (stored emails, electronic files, etc.) and non-content (subscriber and transactional data related to account activities). There is some overlap between these two areas. The following are exceptions that relate to the potential actions of system administrators and network security personnel.

\section{Exceptions for Disclosure of Communications Content}

A person or entity may divulge the contents of a communication...

1) Consent 18 U.S.C. § 2702(b)(3)

with the lawful consent of the originator or an addressee or intended recipient of such communication, or the subscriber in the case of remote computing service...

For example, some companies prohibit the personal use of company email. To enforce this policy, consent from the users is required to allow for monitoring of email. Given this consent, preferably with both a written and signed policy and a banner, compliance checks are possible.

Consent should never be taken as a general permission for system administrators to conduct ad hoc, random monitoring activities. Rather, monitoring activities related to consent should be part of a well-documented process.

2) Provider Exception 18 U.S.C. § 2702(b)(5)

as may be necessarily incident to the rendition of the service or to the protection of the rights or property of the provider of that service.

Products such as email virus scanners may very well fall under this definition, if an application is scanning the contents of stored communications (email) on the mail server before sending it or prior to delivering it. Again, there is a clear nexus between what is being monitored and the threat.

* This example is not intended to be legally authoritative but to frame a legal concept in terms that a system administrator will understand. 
3) Law Enforcement 18 U.S.C. §§ 2702(b)(6)(A)(i)and(ii)

...if the contents were inadvertently obtained by the service provider and appear to pertain to the commission of a crime...

For example, in the examination of email for compliance with company policy, a document is discovered that contains questionable material. The important issue here is that the discovery was incidental, the result of a regular process.*

4) Emergency Situations 18 U.S.C. § 2702(b)(6)(C)

...if the provider reasonably believes that an emergency involving immediate danger of death or serious physical injury to any person requires disclosure of the information without delay...

There are numerous scenarios in which this exception applies. It is often involved in suicide notes, missing children, etc.

\section{Exceptions for Disclosure of Non-Content Customer Records}

...may divulge a record or other information pertaining to a subscriber to or customer of such service (not including the contents of communications covered by subsection...

1) Consent 18 U.S.C. § 2702(c)(2)

with the lawful consent of the customer or subscriber...

2) Provider Exception 18 U.S.C. § 2702(c)(3)

as may be necessarily incident to the rendition of the service or to the protection of the rights or property of the provider of that service...

3) Emergency Situations 18 U.S.C. § 2702(c)(4)

to a governmental entity, if the provider reasonably believes that an emergency involving immediate danger of death or serious physical injury to any person justifies disclosure of the information...

There are numerous scenarios in which this exception applies. It is often involved in suicide notes, missing children, etc. 


\section{Laws that Affect Admissibility of Collection -1}

\section{Federal Rules of Evidence (FRE)}

FRE 801(c)

Hearsay: FRE 801(c): "a statement, other than one made by the declarant while testifying at the trial or hearing, offered in evidence to prove the truth of the matter asserted."

- Computer-generated records

- Computer-stored records

Exception: Rule 803(6) "Records of regularly conducted activity"

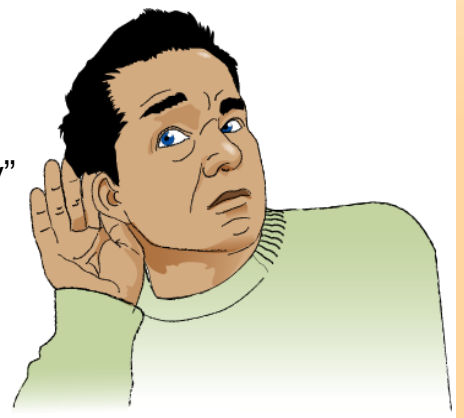

\subsection{Legal Governance Related to Admissibility (Federal Rules of Evidence)}

\subsubsection{Hearsay}

Hearsay is defined by the Federal Rule of Evidence 801(c) as "a statement, other than one made by the declarant while testifying at the trial or hearing, offered in evidence to prove the truth of the matter asserted." Further, according to Rule 802, "Hearsay is not admissible except as provided by these rules or by other rules prescribed by the Supreme Court pursuant to statutory authority or by Act of Congress.”

Searching and Seizing Computers and Obtaining Electronic Evidence in Criminal Investigations, published by the Computer Crime and Intellectual Property Section, Criminal Division, of the United States Department of Justice, is an excellent source for detailed information related to this topic, as well as other general topics dealing with electronic evidence [USDOJ 02]. Its importance to this topic relates to understanding the difference between computer-generated records and computer-stored records. Basically there are two types of computer records: those that "contain the writings of some person or persons and happen to be in electronic form" (i.e., computer-stored records) and those that "contain the 
output of computer programs, untouched by human hands” (i.e., computer-generated records). ${ }^{13}$

It is important to understand the difference between these two types of computer records because computer-stored records can contain hearsay and computer-generated records, which IT professionals are more likely to deal with, cannot. In practice, however, most federal courts have viewed all computer records as potentially containing hearsay. But this view is likely to change:

As the federal courts develop a more nuanced appreciation of the distinctions to be made between different kinds of computer records, they are likely to see that the admission of computer records generally raises two distinct issues. First, the government must establish the authenticity of all computer records by providing "evidence sufficient to support a finding that the matter in question is what its proponent claims." Fed. R. Evid. 901(a). Second, if the computer records are computer-stored records that contain human statements, the government must show that those human statements are not inadmissible hearsay [Kerr 01].

In dealing with computer-generated records, two issues arise: authentication and reliability, which will be explained in subsequent sections.

\subsubsection{Exceptions}

There are twenty-four enumerated exceptions to the Hearsay Rule. Rule 803(6), "records of regularly conducted activity,” deals with issues pertinent to computer forensics. This exception deals with "regularly conducted business activities." This is why an IT policy is so critical regarding network monitoring and incident response. The outcomes from random, ad hoc procedures will not fall under this exception. Rule 803(6) reads as follows:

A memorandum, report, record, or data compilation, in any form, of acts, events, conditions, opinions, or diagnoses, made at or near the time by, or from information transmitted by, a person with knowledge, if kept in the course of a regularly conducted business activity, and if it was the regular practice of that business activity to make the memorandum, report, record, or data compilation, all as shown by the testimony of the custodian or other qualified witness, unless the source of information or the method or circumstances of preparation indicate lack of trustworthiness. The term "business" as used in this paragraph includes business, institution, association, profession, occupation, and calling of every kind, whether or not conducted for profit.

The best example of this would be logging. If a company logs everything as a practice, it is very likely that those logs will be admissible. However, if only partial logging is done and during an incident additional logging is turned on, a question of admissibility may arise.

13 In some cases, records are both computer generated and computer stored. A spreadsheet, for example, could contain both data entered by a person and data generated through calculations done by macros [Kerr 01]. 


\section{Laws that Affect Admissibility of Collection -2}

\section{Authentication}

FRE 901(a) states: The requirement of authentication or identification as a condition precedent to admissibility is satisfied by evidence sufficient to support a finding that the matter in question is what its proponent claims.

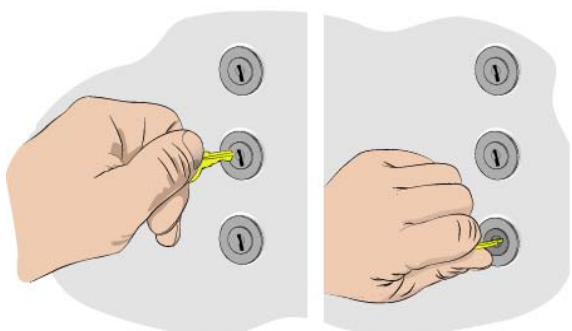

This has more to do with chain of custody than expert testimony

\subsubsection{Authentication}

It is the burden of the person/party attempting to admit a computer-generated record to authenticate that record. Federal Rule of Evidence 901(a) states the following:

The requirement of authentication or identification as a condition precedent to admissibility is satisfied by evidence sufficient to support a finding that the matter in question is what its proponent claims.

To authenticate a computer record, a person who acquired the record or caused the record to be generated can testify to the authenticity of the record (i.e., say that the record is what it purports to be). An example of this is when a system administrator records the open connections on a system by running a netstat command and has that information saved on a diskette. This same system administrator would need to authenticate this diskette at court by testifying that he or she ran the command that caused the output and, further, that the output was saved to that diskette. It is not important or required that the system administrator have programming knowledge and understand how the netstat command works or to be able to provide analysis of the results of the netstat command. All that is required is that the system administrator has first-hand knowledge, i.e., that he or she ran the command and generated the output that was then saved to the diskette.

In addition, chain of custody plays a significant role in the process of authentication. It is not enough to just testify after the fact about what was collected. Having a process in place that will track collected information and ensure that it is preserved and not tampered with is also required. 


\title{
Laws that Affect Admissibility of Collection -3
}

\section{Reliability: Computer Records/Processes}

\author{
Technical code analysis not required
}

Verification of output

Normal course of business

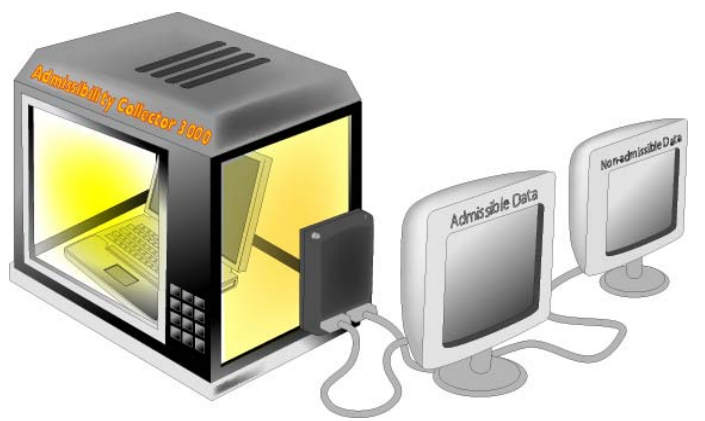

๑) 2005 Carnegie Mellon University

Module 1: Cyber Law

\subsubsection{Reliability}

Another issue that IT personnel should be prepared to deal with is the reliability of the computer programs that generate the computer records. This area is of particular interest to IT staff, since it is where software that is used in the collection of information related to an incident may be challenged.

For the purpose of establishing reliability, computer programs are distinguished by whether they are or are not run in the normal course of business. An example of programs run in the normal course of business is RADIUS servers at an ISP supporting dial-up users. RADIUS servers provide several functions related to an ISP's business operations. For example, a RADIUS server would be used to maintain logs of when users dial in to access the network. The ISP normally uses this information for billing purposes, but the same information may become important in the event of an incident. When the information is used to support an incident, the fact the program that generated the information was used in the normal course of business gives it reliability [Kerr 01].

The more difficult situation in terms of reliability is when programs are used to collect information that are not run in the normal course of business. The guidance from the courts is more complicated in this area, as indicated in this excerpt from Searching and Seizing Computers and Obtaining Electronic Evidence in Criminal Investigations:

The courts have indicated that the government can overcome this challenge so long as "the government provides sufficient facts to warrant a finding that the records are trustworthy and the opposing party is afforded an opportunity to 
inquire into the accuracy thereof[.]" United States v. Briscoe, 896 F.2d 1476, 1494 (7th Cir. 1990). See also Liebert, 519 F.2d at 547; DeGeorgia, 420 F.2d. at 893 n.11. Compare Fed. R. Evid. 901(b)(9) (indicating that matters created according to a process or system can be authenticated with "[e]vidence describing a process or system used...and showing that the process or system produces an accurate result")...the government may need to disclose "what operations the computer had been instructed to perform [as well as] the precise instruction that had been given" if the opposing party requests. Dioguardi, 428 F.2d at 1038 [USDOJ 02].

How does this translate into working knowledge for IT staff? Module 2, "File Systems, Duplication, Wiping, and Trusted Tools," will describe some of the technical implications of dealing with reliability issues. 


\section{Laws that Affect Admissibility of Collection -4}

\section{Best Evidence:}

FRE 1001(3): If data is stored in a computer or similar device, any printout or other output readable by sight, shown to reflect the data accurately, is an "original".

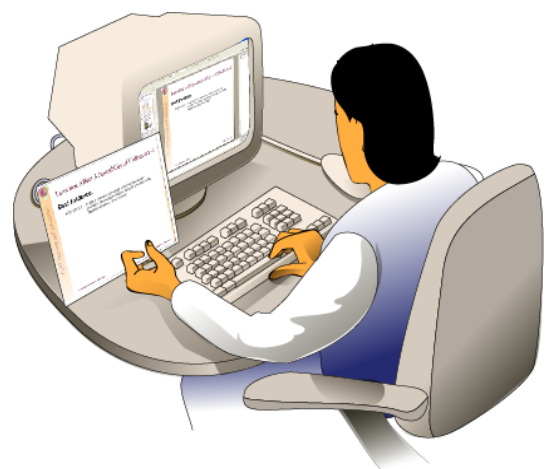

\subsubsection{The Best Evidence Rule}

The Best Evidence rule (Federal Rule of Evidence 1002) states that to prove the content of writing, recording, or photograph, the original writing, recording, or photograph is ordinarily required. Federal Rule of Evidence 1001(3) defines “original”:

If data are stored in a computer or similar device, any printout or other output readable by sight, shown to reflect the data accurately, is an "original".

The courts have long recognized that at times it may be both impractical and unreasonable to require originals, which might be entire computers or large volumes of data, for information that otherwise could be represented in a chart or graph. In fact, in the $9^{\text {th }}$ Circuit the courts require that during seizures of computers, on-site imaging must be done, leaving the original behind and taking only a "copy," that is, a true bit by bit forensic image. 


\section{Summary}

1. System administrator as the first responder to a security incident Authority to collect/admissibility of collection

2. Wiretap Act I Pen/Trap and Trace

Layer $5+$ is the content portion of the packet

Layers 2, 3, and 4 are the non-content portion of the packet

3. Hearsay and Computer Records

"Regular Course of Business"

4. Stored Communication

Applied to public providers only

5. 4th and 5th Amendments

5th Amendment-Cannot be compelled to provide private key if memorized

4th Amendment-Some protection outside of the home

(c) 2005 Carnegie Mellon University

Module 1: Cyber Law

\subsection{Summary}

The five points above are the key concepts that should be understood from this module. 


\section{Review}

Where is the technical difference between content and non-content in the OSI Model?

What cyber law governs the usage of tools like sniffers?

How many people are required for "consent" to be given?

What aspect of electronic communication does the Pen/Trap and Trace Statue cover?

What is the important difference between computergenerated and computer-stored records in terms of cyber law?

\subsection{Review}

Q. Where is the technical difference between content and non-content in the OSI Model?

A. Between Layers 4 and 5 .

Q. What cyber law governs the usage of tools like sniffers?

A. The Wiretap Act.

Q. How many people are required to give "consent” under federal law?

A. Only one.

Q. What aspect of electronic communication does the Pen/Trap and Trace Statute cover?

A. The non-content portion of real-time communications.

Q. What is the important difference between computer-generated and computer-stored records in terms of cyber law?

A. Computer-generated records contain no human statements and computer-stored records may contain human statements. 


\section{Module 2: Understanding File Systems and Building a First Responder Toolkit}

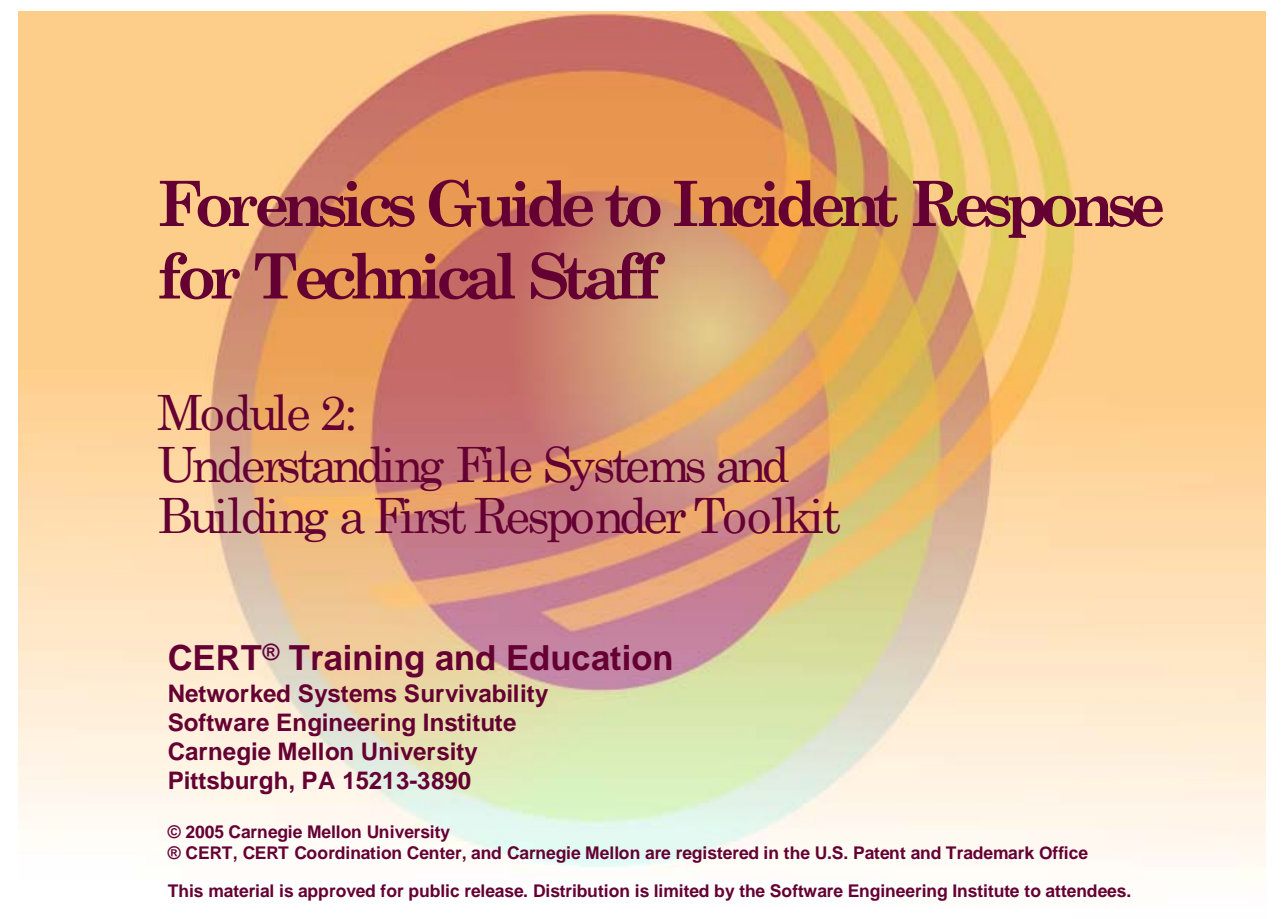

\subsection{Introduction}

Module 2 consists of two distinct sections: Understanding File Systems, and Methodologies for Building a First Responder Toolkit.

The first section, Understanding File Systems, is an introduction to technical concepts and terms important for later modules. Its intent is to provide the novice with a foundation and the knowledgeable with a baseline cursory review. The first portion provides an overview of how the operating system (OS) interacts with physical storage media in creating and modifying files. Most storage occurs on a computer's internal hard drive and a basic understanding of hard drive design provides insight into the advantages and limitations of file systems. A cursory look at the similarities between Windows and Linux is highlighted with a brief discussion of each OS's characteristics. The last portion of this section addresses common forensic nomenclature. 
The second section outlines a best practice methodology for creating a trusted first responder tool kit that system and network administrators can use when responding to security events or system anomalies on live systems. The goal of this step-by-step, repeatable, and welldocumented approach is to ensure all information is collected using sound forensic practices. 


\title{
Introduction to File System Architecture
}

\author{
Hard disk drives \\ File systems \\ Windows \\ UNIX/LinuX
}

\section{Boot sequence}

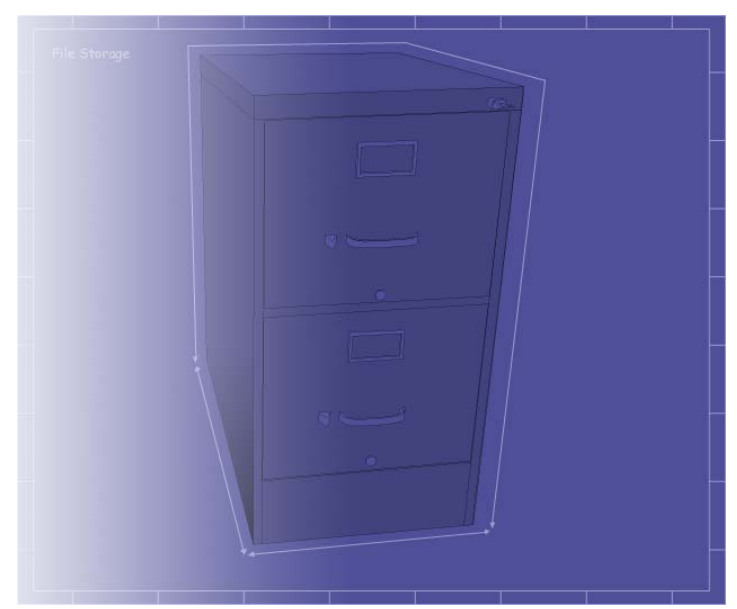

Module 2: Understanding File Systems and Building a First Responder Toolkit

\subsection{File System Architecture}

File system design concepts and technical details fill up large volumes of textbooks, so a detailed discussion on this topic is well beyond the scope of this introductory handbook. This section will provide a basic understanding of the most popular storage media, the hard drive, and how its design interacts with an OS's file system. This overview of the Windows and Linux file systems will provide the foundation for the next two modules dealing with collection. Additionally, the vocabulary covered in the next few pages contributes to the technical staff's communication with a professional cyber investigator.

Because of the large array of different file systems, especially with regard to open-source OSs like Linux, you are encouraged to expand your knowledge outside the boundaries of this handbook for your particular networks. An understanding of what is expected in the normal use of a file system (where typically certain files are placed, what default directories are named, etc.) assists in the ability to notice anomalies - which is the first step toward incident detection. 


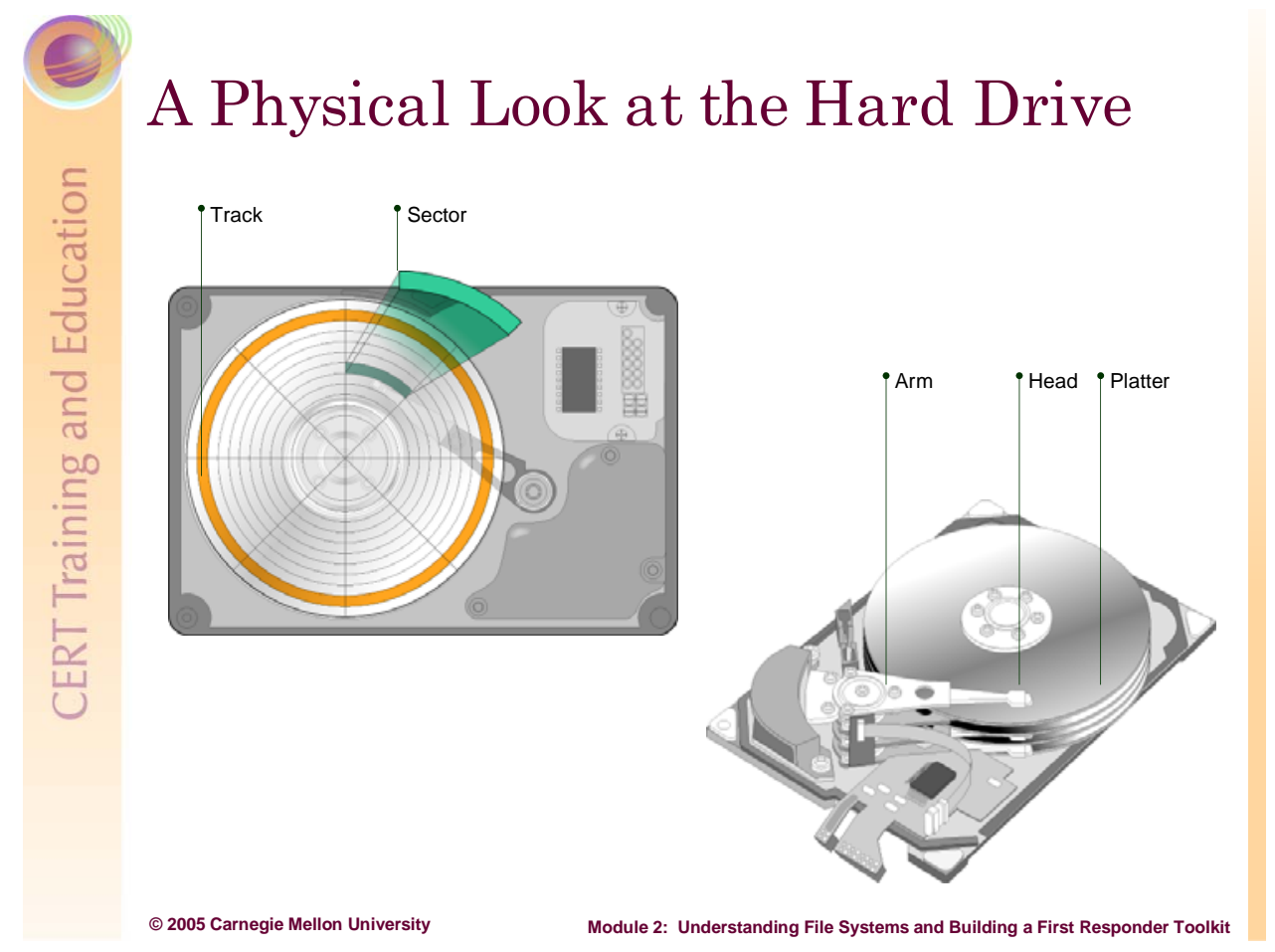

\subsubsection{Physical Look at the Hard Drive}

To understand how file systems work on different OSs, you must be familiar with the physical organization of the hard disk drive. A hard drive is like a record player, except that the hard drive can handle multiple stacks of records. On a hard drive, the magnetic media are called platters, and data is written on them by a read/write arm that has a head (analogous to a needle).

Hard drives consist of several platters with heads inserted between them that can read on one or both sides. The data on each platter is physically organized into tracks and sectors. A track is an individual concentric circle on the platter. A combination of tracks is called a cylinder. A sector divides a track and typically holds 512 bytes.

By knowing the attributes of a hard disk drive, you can determine its maximum storage capacity.

Bytes $=\mathrm{C} / \mathrm{D} * \mathrm{H} / \mathrm{C} * \mathrm{~S} / \mathrm{T} * 512$ bytes

$\mathrm{C}$ is the number of cylinders

$\mathrm{T}$ is the number of tracks (track numbering starts with 0 , not 1 )

$\mathrm{S}$ is the number of sectors

For more details, visit the following Web sites:

- $\quad$ http://www.pcguide.com/ref/hdd/

- http://www.howstuffworks.com/hard-disk.htm 


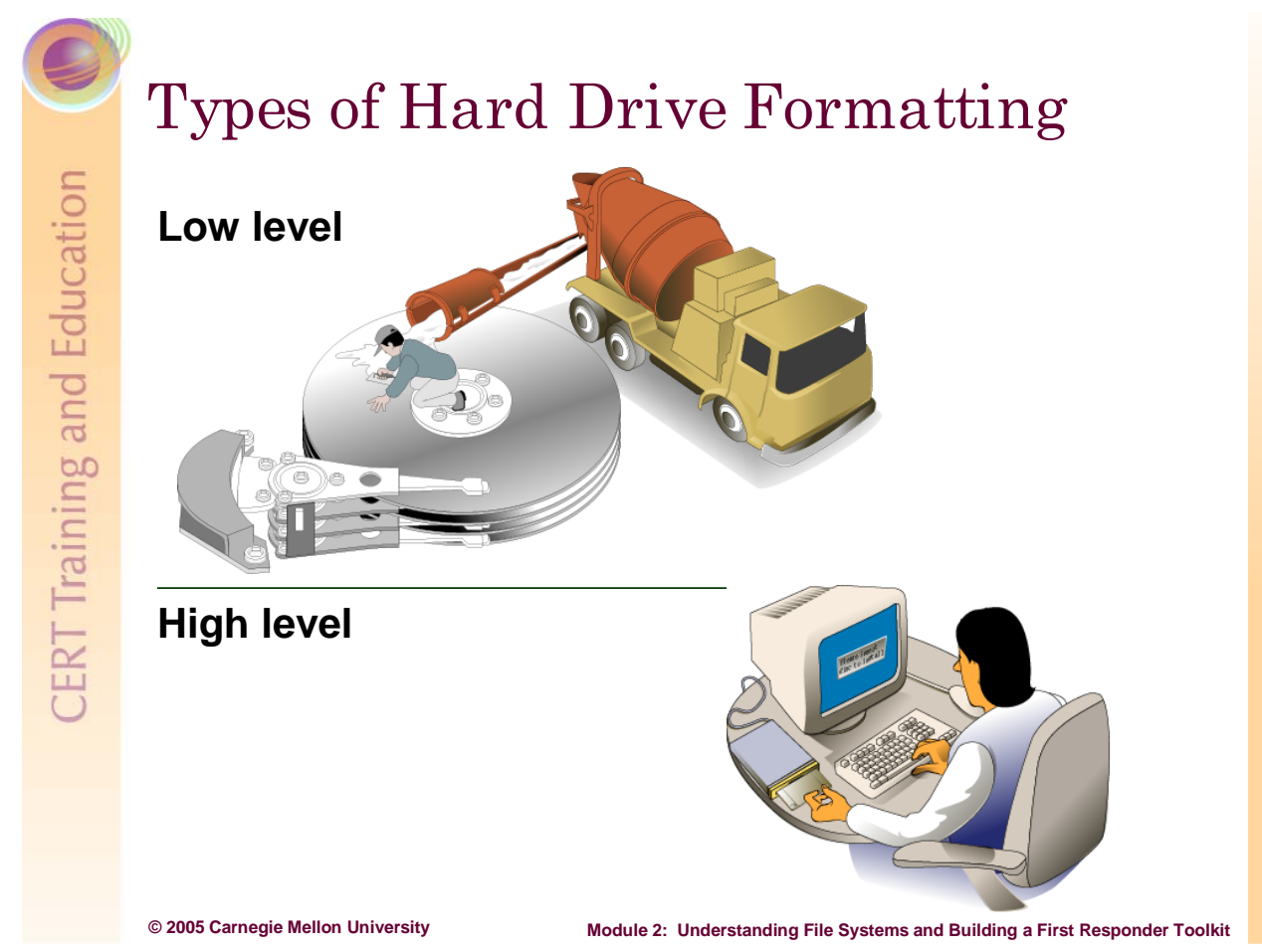

\subsubsection{Types of Hard Drive Formatting}

The two types of hard drive formatting are low and high level. Low-level formatting creates the track and sectors on the drive. These tracks and sectors form the physical blocks of storage of 512 bytes each.

High-level formatting is file system specific, including Microsoft (DOS, FAT, and NTFS) and Open Source Initiative (OSI) varieties (ext2, ReiserFS, and XFS).

Low-level formatting is done by the hard drive vendor. High-level formatting is done when the OS is installed.

High-level formatting creates the hard drive's file system and allows the OS to store files by dividing them into smaller pieces and saving them in separate clusters (a grouping of sectors) on the disk. The OS uses this file system to keep track of the placement and sequence of each piece and to identify which sectors on the disk are free and available for new files. The computer can then assemble the different pieces when a file is viewed or executed. 


\title{
Why Understand File Systems?
}

\author{
Different OS, different file \\ systems
}

\section{Poor documentation}

File location

Hidden data

File deletion

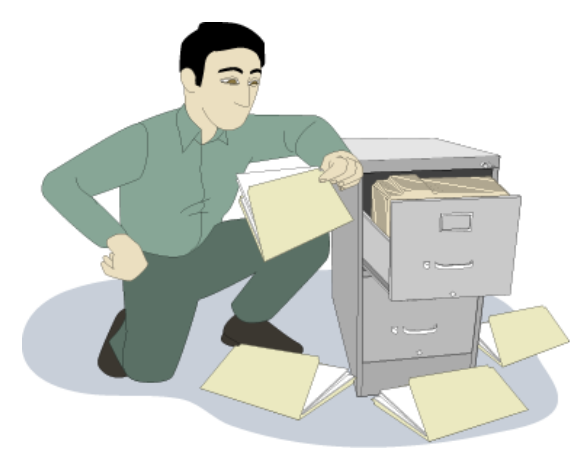

(๖) 2005 Carnegie Mellon University

Module 2: Understanding File Systems and Building a First Responder Toolkit

\subsubsection{Importance of File Systems}

A file system has two basic functions that impact the computer's performance:

- mapping physical spaces on the drive to logical addresses that comprise files

- $\mathrm{read} / \mathrm{write}$ capability to open, change, and delete files

Understanding how these functions work on different file systems is the foundation for responding to a security incident. Most file systems are related directly to a particular OS, although some OSs combine file systems.

To discover where files are located and how they are distributed, you need to know how to access and modify system settings when necessary. This is especially important because files can be hidden.

Typically when a user deletes a file, the file system does not permanently erase (wipe) the file from the hard drive. It simply creates a flag that tells the OS that the sector can be reused. Knowing how to rebuild files from the file system is one of the most important skills of the forensic examiner.

The forensic examiner has access to deleted files and to files contained in swap space, which is part of the virtual memory created on the hard drive by the OS. Swap space files are described later in this module. 


\section{Windows File Structure}

Disk partitioning

Cluster versus sector

File allocation table (FAT)

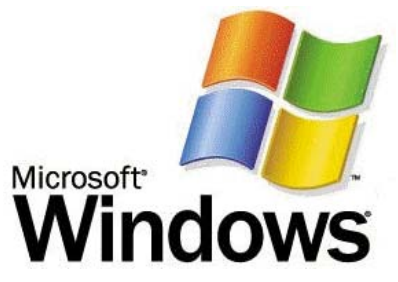

New technology file system (NTFS)

Modified, accessed, created (MAC)

๑) 2005 Carnegie Mellon University

Module 2: Understanding File Systems and Building a First Responder Toolkit

\subsubsection{Understanding Windows File Structure}

On a physical hard disk, more then one logical section may exist. Dividing a physical drive into separate pieces is called partitioning. The partitions of logical drives are traditionally labeled by the file system as C, D, E, and so on.

Microsoft Windows is the predominate OS for user workstations, and has gained significant momentum in server applications. To understand Microsoft's file structure, we must start by defining some storage terms: clusters, file allocation tables (FAT), and the new technology file system (NTFS).

The hard drive's sectors, as previously described, are further grouped by the Microsoft file system into clusters. Clusters contain groups of sectors. These clusters then form larger data groups to make a single larger addressable storage unit. By combining sectors into clusters, the file system reduces the overhead to write and read files to the disk as it has to keep track of fewer unique storage areas. Usually the number of sectors to a cluster can be seen below for the Windows XP OS. The following excerpt is from the Microsoft Windows XP Professional Resource Kit Documentation [Microsoft 04].

A cluster (or allocation unit) is the smallest amount of disk space that can be allocated to hold a file. All file systems used by Windows XP Professional organize hard disks based on cluster size, which is determined by the number of sectors that the cluster contains. For example, on a disk that uses 512-byte sectors, a 512-byte cluster contains one sector, whereas a 4-KB cluster contains eight sectors. 
The two primary Windows file systems are FAT and NTFS. Both will be described later.

FAT16, FAT32, and NTFS each use different cluster sizes depending on the size of the volume, and each file system has a maximum number of clusters it can support. When the cluster size is smaller, the disk stores information more efficiently because unused space within a cluster cannot be used by other files. And when more clusters are supported, you can create and format larger volumes by using a particular file system.

Table 1 compares FAT16, FAT32, and NTFS volume and default cluster sizes.

Table 1: $\quad$ Default Cluster Sizes for Volumes with Windows XP Professional File Systems

\begin{tabular}{|l|l|l|l|}
\hline Volume Size & FAT16 Cluster Size & FAT32 Cluster Size & NTFS Cluster Size \\
\hline 7 MB-16 MB & 2 KB & Not supported & 512 bytes \\
\hline 17 MB-32 MB & 512 bytes & Not supported & 512 bytes \\
\hline 33 MB-64 MB & 1 KB & 512 bytes & 512 bytes \\
\hline 65 MB-128 MB & 2 KB & 1 KB & 512 bytes \\
\hline 129 MB-256 MB & 4 KB & $2 \mathrm{~KB}$ & 512 bytes \\
\hline 257 MB-512 MB & $8 \mathrm{~KB}$ & $4 \mathrm{~KB}$ & 512 bytes \\
\hline 513 MB-1,024 MB & $16 \mathrm{~KB}$ & $4 \mathrm{~KB}$ & 1 KB \\
\hline 1,025 MB-2 GB & $32 \mathrm{~KB}$ & $4 \mathrm{~KB}$ & $2 \mathrm{~KB}$ \\
\hline 2 GB-4 GB & $64 \mathrm{~KB}$ & $4 \mathrm{~KB}$ & $4 \mathrm{~KB}$ \\
\hline 4 GB-8 GB & Not supported & $4 \mathrm{~KB}$ & $4 \mathrm{~KB}$ \\
\hline 8 GB-16 GB & Not supported & $8 \mathrm{~KB}$ & $4 \mathrm{~KB}$ \\
\hline 16 GB-32 GB & Not supported & $16 \mathrm{~KB}$ & $4 \mathrm{~KB}$ \\
\hline 32 GB-2 terabytes & Not supported & Not supported & $4 \mathrm{~KB}$ \\
\hline
\end{tabular}

A very helpful feature of Windows file systems is the details concerning MAC times, which stands for modified, accessed, and created. Date and time properties are appended to files as they are created, accessed, and modified. Examine a file's MAC times for additional information about the history of the file, including a user's interaction with it. 


\section{FAT: File Allocation Table}

\section{Microsoft original}

\section{Table}

\section{Three versions}

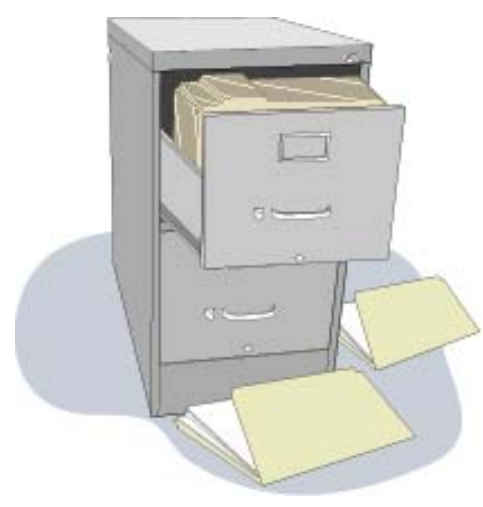

Module 2: Understanding File Systems and Building a First Responder Toolkit

\subsubsection{FAT: File Allocation Table}

The following is from Microsoft's Intellectual Property and Licensing Group introduction to FAT [Microsoft 03a]:

The first FAT file system was developed by Microsoft in 1976. That system was based on the BASIC programming language and allowed programs and data to be stored on a floppy disk. Since that time, the FAT file system has been improved upon multiple times to take advantage of advances in computer technology, and to further refine and enrich the FAT file system itself.

Today, the FAT file system has become the ubiquitous format used for interchange of media between computers, and, since the advent of inexpensive, removable flash memory, also between digital devices. The FAT file system is now supported by a wide variety of OSs running on all sizes of computers, from servers to personal digital assistants. In addition, many digital devices such as still and video cameras, audio recorders, video game systems, scanners, and printers make use of FAT file system technology.

The FAT database table contains the filenames, directory names, MAC times, cluster number, and assorted attributes (hidden, read-only, etc.) of disk's files. This table resides at the outermost portion of the disk with FAT32 offering a redundant copy in the event the primary suffers damage. The recovery of the redundant table is crucial if malicious activity makes the primary table unreadable. 
For a history of the FAT file system, visit the Microsoft Web site [Microsoft 03b].

Figure 2 [Stoffregen 03] shows the overall logical layout of the FAT32 file system, with the allocation tables, primary and backup (labeled FAT \#1 and \#2 respectively), written directly behind the volume ID and boot sector (contained in the reserved sectors).

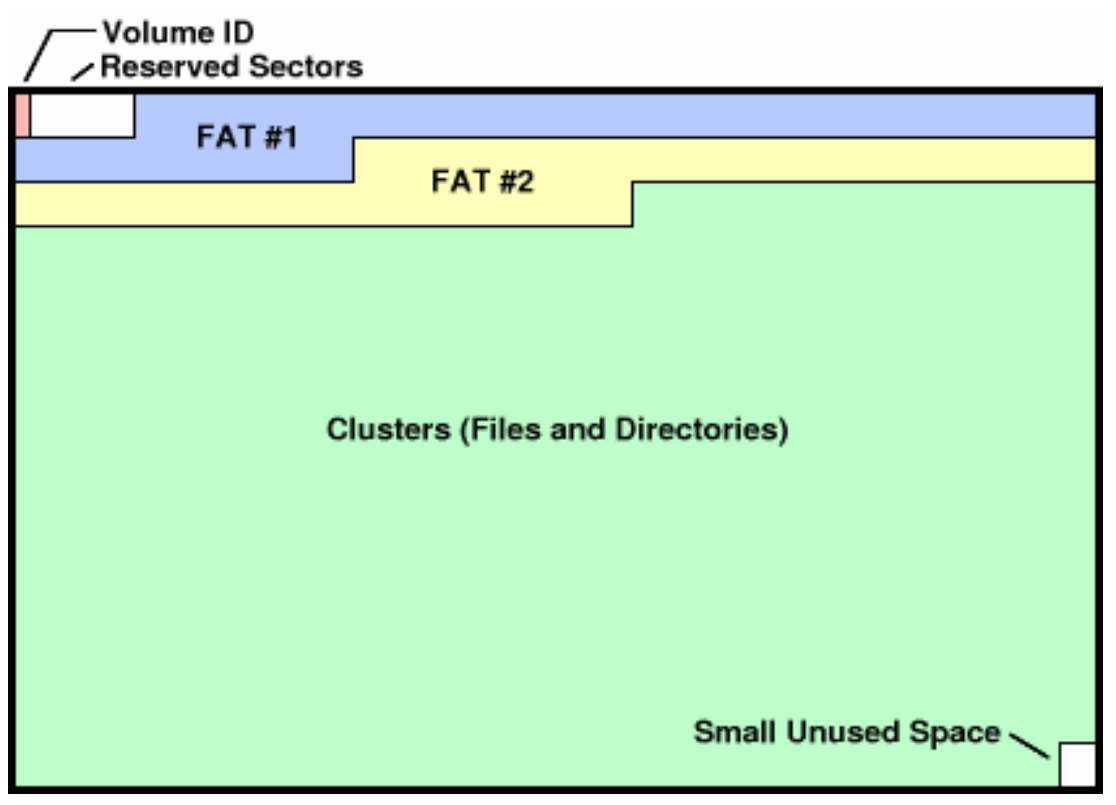

Figure 2: Logical Layout of the FAT32 File System 


\section{NTFS: New Technology File System}

Master file table (MFT)

Access rights

Encryption

Larger volume sizes

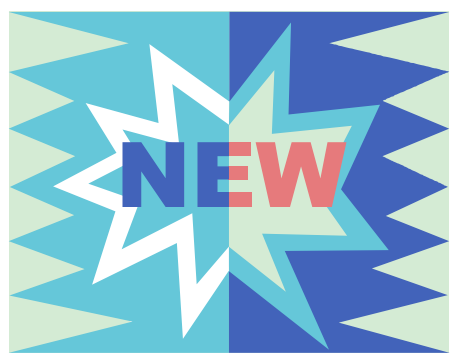

\section{Multiple data streams}

\subsubsection{NTFS: New Technology File System}

NTFS is currently the primary file system used by Windows XP. It was first introduced with Windows NT.

The Microsoft Windows XP Professional Resource Kit Documentation [Microsoft 04] is a comprehensive NTFS resource. The following excerpts are from the site.

NTFS replaces the FAT and uses a master file table (MFT), which is the first file on the disk. Records within the MFT are called meta-data and this contains information on all files located on the disk, including system files. A key advancement is the way files and directories are both stored on the disk with attributes that include security information. At format the MFT assigns logical cluster numbers (LCN) to the disk's entire partition. These LCNs allow the OS to read and write data on the disk. Each LCN is similarly linked to a virtual cluster number (VCN) which allows files to extend beyond across the free disk space area of the hard drive. 
On NTFS volumes you can set permissions on files and folders that specify which groups and users have access, and what level of access is permitted. NTFS file and folder permissions apply to users on the local computer and to users accessing the file or folder over the network. File and folder permissions are maintained in discretionary access control lists.

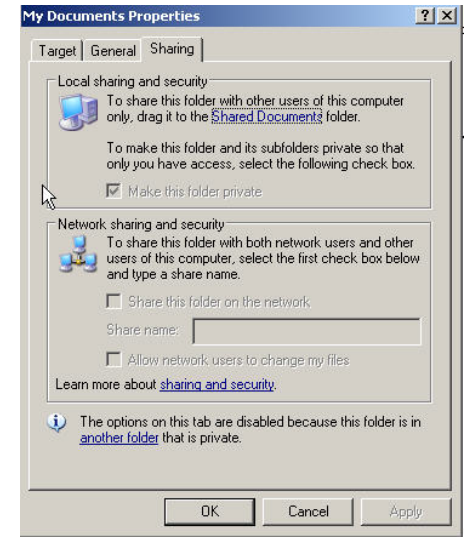

\section{Encryption}

The encrypting file system (EFS) uses symmetric key encryption in conjunction with public key technology to protect files and folders. Encryption ensures that only the authorized users and designated recovery agents of that file or folder can access it. Users of EFS are issued a digital certificate with a public key and a private key pair. EFS uses the key set for the user who is logged on to the local computer where the private key is stored.

Users work with encrypted files and folders just as they do with any other files

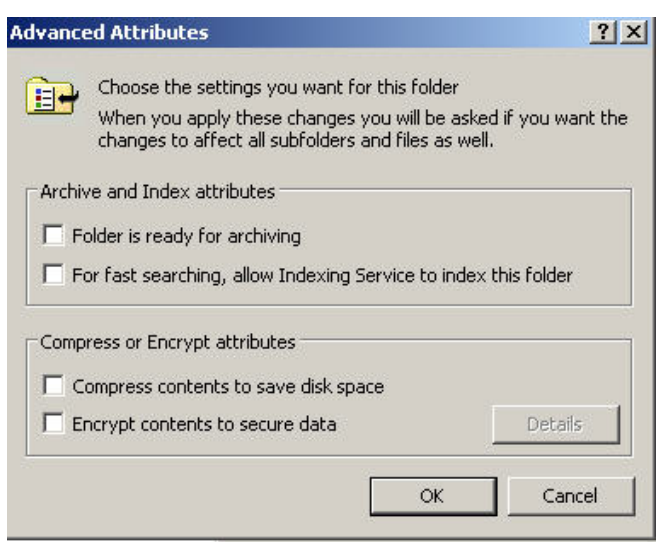
and folders. Encryption is transparent to any authorized users; the system decrypts the file or folder when the user opens it. When the file is saved, encryption is reapplied. However, intruders who try to access the encrypted files or folders receive an "Access denied" message if they try to open, copy, move, or rename the encrypted file or folder.

\section{Larger Volume Size}

The maximum NTFS volume size as implemented in Windows XP Professional is 232 clusters minus 1 cluster, which is approximately 256 terabytes with a max individual file size of about 16 terabytes. Under FAT32, the maximum volume size was 32Gig with a 4 Gig file. This has considerable impact on storage requirement for making forensic duplications and putting together fragmented files.

\section{$\underline{\text { Multiple Data Streams }}$}


A data stream is a sequence of bytes. An application populates the stream by writing data at specific offsets within the stream. The application can then read the data by reading the same offsets in the read path. Every file has a main, unnamed stream associated with it, regardless of the file system used. However, NTFS supports additional named data streams in which each data stream is an alternate sequence of bytes as illustrated in the figure. Applications can create additional named streams and access the streams by referring to their names. This feature permits related data to be

Streamexample.doc
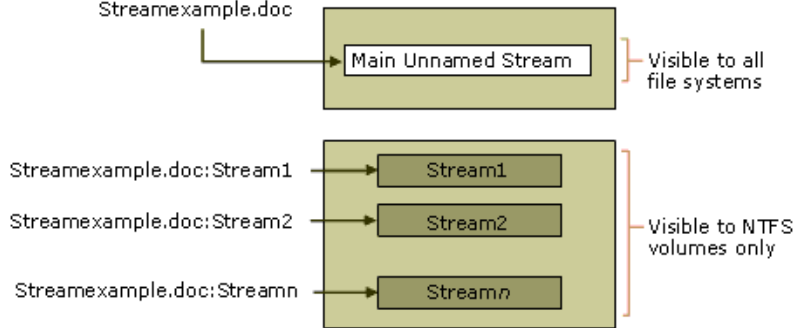

managed as a single unit. For example, a graphics program can store a thumbnail image of bitmap in a named data stream within the NTFS file containing the image.

A forensic examiner is particularly interested in these multiple data streams since they can hide data either intentionally or by coincidence. The data stream is an additional data attribute of a file.

\section{$\underline{\text { Cluster Size }}$}

As described previously, the cluster size has also significantly increased with NTFS. 


\section{Windows Registry}

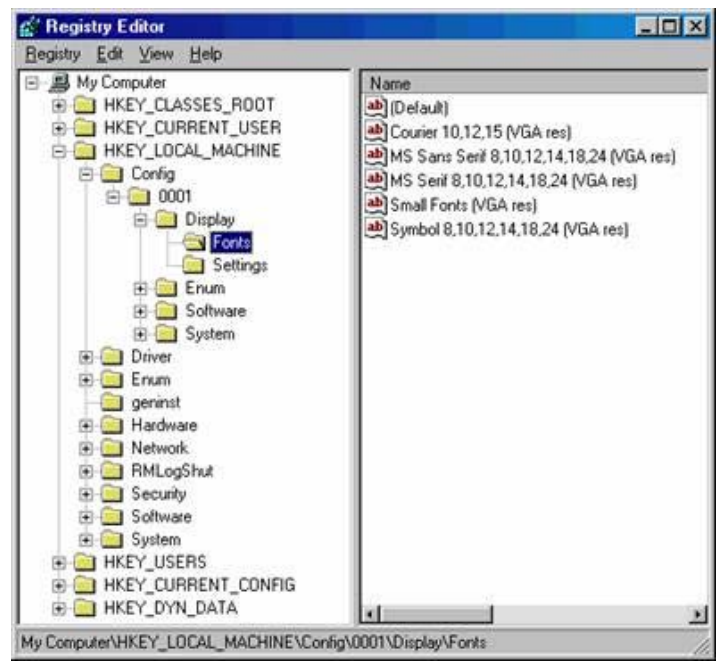

๑) 2005 Carnegie Mellon University

Module 2: Understanding File Systems and Building a First Responder Toolkit

\subsubsection{Windows Registry}

The registry [Russinovich 97] has been the centralized configuration database for Windows and supported applications since Windows 95s' introduction. From the Windows registry, a forensics examiner can discover hardware and software configuration, user preferences, initial system settings, and even logon and password information. Windows artifact analysis is quickly becoming an important part of forensic discovery, so you must understand how altering the registry settings can corrupt the system's evidence collection and documentation.

Over time, as more and more installation and removal program settings are saved, the registry becomes cluttered. The following freeware utilities can delete these unused or old registry keys and clean up your computer:

- Jv16 Power Tools v1.4.1.248

http://www.pcworld.com/downloads/file_description/0,fid,22765,00.asp

- RegClean v4.1a

http://www.pcworld.com/downloads/file_description/0,fid,4666,RSS,RSS,00.asp 


\section{Swap File, Slack, and Unallocated Space}

\section{Swap file}

RAM slack (blue) and file slack (red)

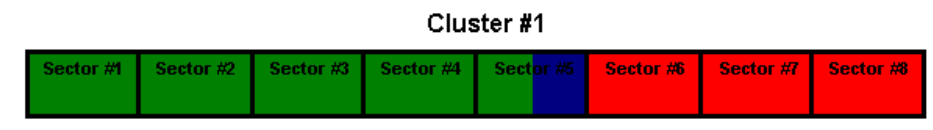

4,096 bytes per cluster (4K)

512 bytes per sector

File is green

\section{Unallocated disk space}

University of North Texas, CJUS 5120, Cybercrime and Digital Forensics.

http://www.unt.edu/cjus/Course_Pages/CJUS_5120 (2004)

๑) 2005 Carnegie Mellon University

Module 2: Understanding File Systems and Building a First Responder Toolkit

\subsubsection{Swap File, Slack, and Unallocated Space}

\subsubsection{Swap File}

The swap file is a hidden system file that is used for virtual memory when there is not enough physical memory to run programs. Space on the hard drive is temporarily swapped with the RAM as programs are running. This swap file contains portions of all documents and other material a user produces while using the computer. In Windows NT/XP systems, the swap file is named pagefile.sys; on older Windows 9X systems, it is named win386.swp. The key for a forensic examiner is that a user might cover his tracks by wiping all files but this one, which he may not know exists.

\subsubsection{Slack Space}

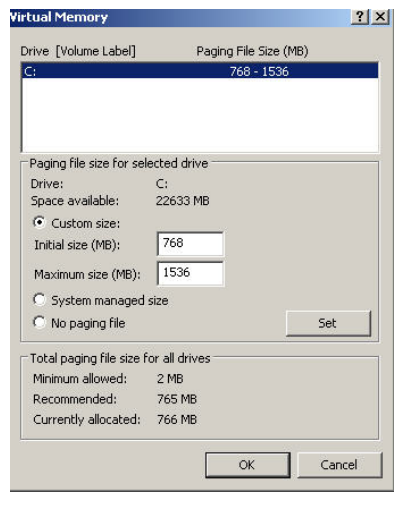

As shown in the slide's graphic, slack space results when file systems create a cluster (Windows) or block (Linux) but do not necessarily use the entire fixed length space that was allocated. In this case, file information from previous use is still available, long after deletions and rewrites. At the very least, viewing these clusters can prove to a forensic examiner that a certain type of file might have existed. A significant advantage of NTFS is that it generates much less slack space. 


\subsubsection{Unallocated Space}

When a user deletes a file, it is flagged as no longer needed, but it remains on the system until it is overwritten. The remaining files are in unallocated disk space, where clusters/blocks are not assigned but may contain data. A forensic software tool can identify and restore these files. 


\section{Linux}

\section{Many different file systems}

i-nodes

Iproc

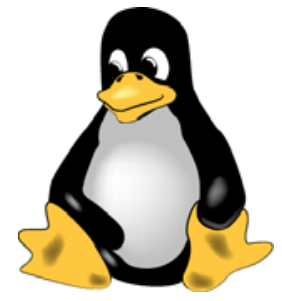

() 2005 Carnegie Mellon University

Module 2: Understanding File Systems and Building a First Responder Toolkit

\subsubsection{Linux File System Basics}

The following excerpt is from a paper describing the Linux basic file system first published in the Proceedings of the First Dutch International Symposium on Linux (http://e2fsprogs.sourceforge.net/ext2intro.html):

\section{Basic File System Concepts}

Every Linux file system implements a basic set of common concepts derivated from the UNIX OS. Files are represented by inodes, and directories are simply files containing a list of entries and devices can be accessed by requesting I/O on special files.

\section{$\underline{\text { Inodes }}$}

Each file is represented by a structure, called an inode. Each inode contains the description of the file: file type, access rights, owners, timestamps, size, pointers to data blocks. The addresses of data blocks allocated to a file are stored in its inode. When a user requests an I/O operation on the file, the kernel code converts the current offset to a block number, uses this number as an index in the block addresses table and reads or writes the physical block. This figure represents the structure of an inode: 


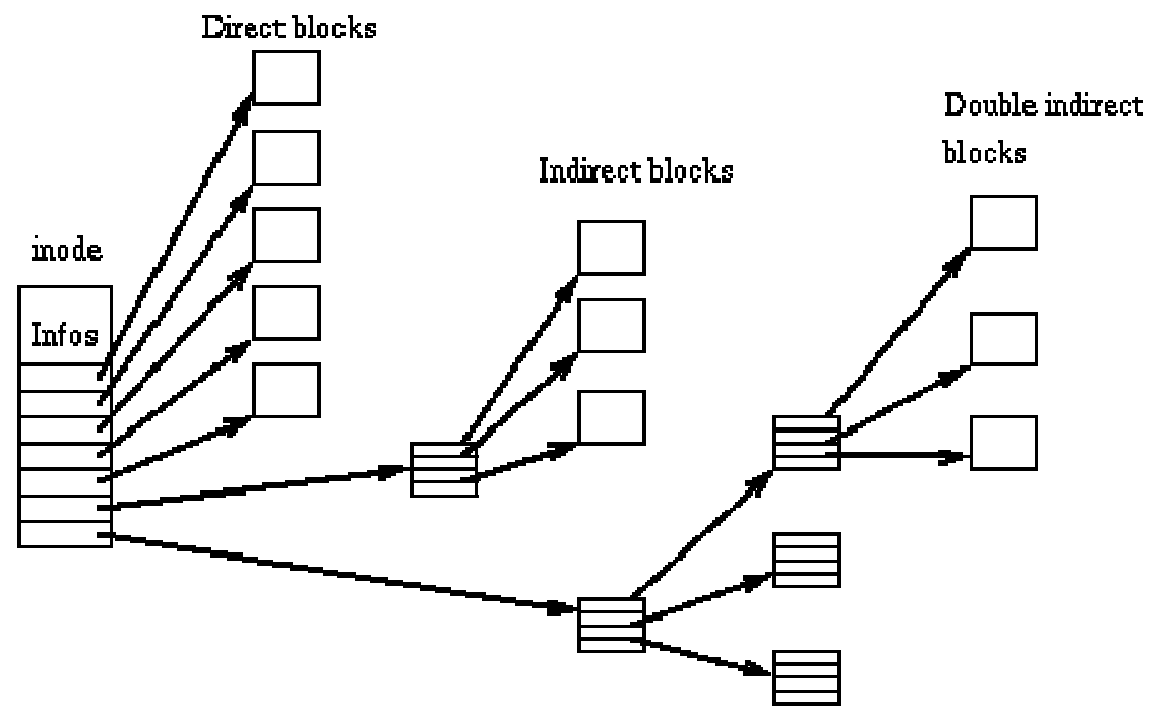

\section{$\underline{\text { Directories }}$}

Directories are structured in a hierarchical tree. Each directory can contain files and subdirectories.

Directories are implemented as a special type of files. Actually, a directory is a file containing a list of entries. Each entry contains an inode number and a file name. When a process uses a pathname, the kernel code searches in the directories to find the corresponding inode number. After the name has been converted to an inode number, the inode is loaded into memory and is used by subsequent requests.

Inode table

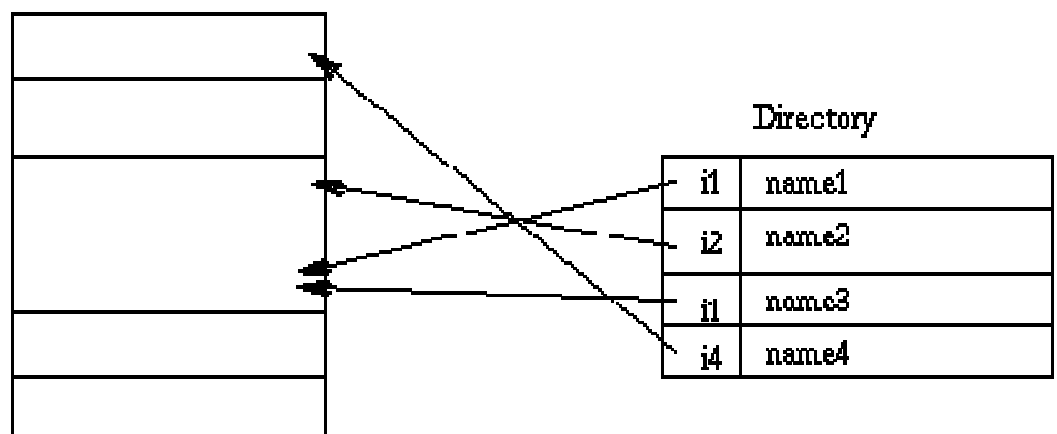

\section{$\underline{\text { Links }}$}

UNIX file systems implement the concept of link. Several names can be associated with an inode. The inode contains a field containing the number associated with the file. Adding a link simply consists of creating a directory entry, where the inode number points to the inode, and in incrementing the links 
count in the inode. When a link is deleted, i.e. when one uses the rm command to remove a filename, the kernel decrements the links count and deallocates the inode if this count becomes zero.

This type of link is called a hard link and can only be used within a single file system: it is impossible to create cross-file system hard links. Moreover, hard links can only point on files: a directory hard link cannot be created to prevent the apparition of a cycle in the directory tree.

Another kind of link exists in most UNIX file systems. Symbolic links are simply files which contain a filename. When the kernel encounters a symbolic link during a pathname to inode conversion, it replaces the name of the link by its contents, i.e. the name of the target file, and restarts the pathname interpretation. Since a symbolic link does not point to an inode, it is possible to create cross-file systems symbolic links. Symbolic links can point to any type of file, even on nonexistent files. Symbolic links are very useful because they don't have the limitations associated to hard links. However, they use some disk space, allocated for their inode and their data blocks, and cause an overhead in the pathname to inode conversion because the kernel has to restart the name interpretation when it encounters a symbolic link.

\section{$\underline{\text { Device Special Files }}$}

In UNIX-like OSs, devices can be accessed via special files. A device special file does not use any space on the file system. It is only an access point to the device driver.

Two types of special files exist: character and block special files. The former allows I/O operations in character mode while the later requires data to be written in block mode via the buffer cache functions. When an I/O request is made on a special file, it is forwarded to a (pseudo) device driver. A special file is referenced by a major number, which identifies the device type, and a minor number, which identifies the unit.

\section{The Virtual File System}

\section{$\underline{\text { Principle }}$}

The Linux kernel contains a virtual file system layer which is used during system calls acting on files. The VFS is an indirection layer which handles the file oriented system calls and calls the necessary functions in the physical file system code to do the I/O.

This indirection mechanism is frequently used in UNIX-like OSs to ease the integration and the use of several file system types. 
When a process issues a file oriented system call, the kernel calls a function contained in the VFS. This function handles the structure independent manipulations and redirects the call to a function contained in the physical file system code, which is responsible for handling the structure dependent operations. File system code uses the buffer cache functions to request I/O on devices. This scheme is illustrated in this figure:

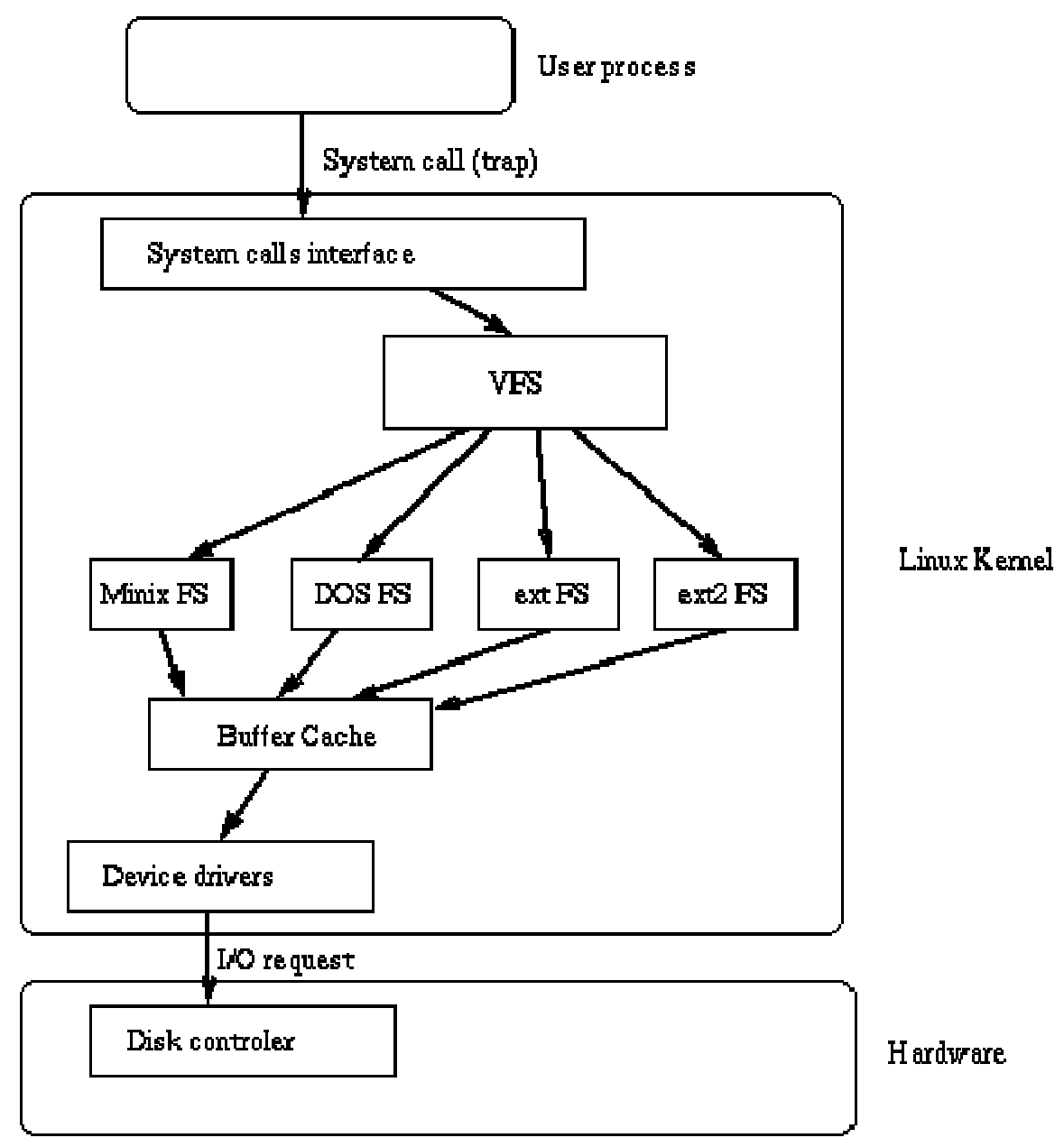

\section{The VFS Structure}

The VFS defines a set of functions that every file system has to implement. This interface is made up of a set of operations associated to three kinds of objects: file systems, inodes, and open files.

The VFS knows about file system types supported in the kernel. It uses a table defined during the kernel configuration. Each entry in this table describes a file system type: it contains the name of the file system type and a pointer on a function called during the mount operation. When a file system is to be mounted, the appropriate mount function is called. This function is responsible for reading the superblock from the disk, initializing its internal variables, and returning a 
mounted file system descriptor to the VFS. After the file system is mounted, the VFS functions can use this descriptor to access the physical file system routines.

A mounted file system descriptor contains several kinds of data: information that is common to every file system type, pointers to functions provided by the

physical file system kernel code, and private data maintained by the physical file system code. The function pointers contained in the file system descriptors allow the VFS to access the file system internal routines.

Two other types of descriptors are used by the VFS: an inode descriptor and an open file descriptor. Each descriptor contains information related to files in use and a set of operations provided by the physical file system code. While the inode descriptor contains pointers to functions that can be used to act on any file (e.g., create, unlink), the file descriptors contains pointer to functions which can only act on open files (e.g., read, write). 


\section{Boot Sequence}

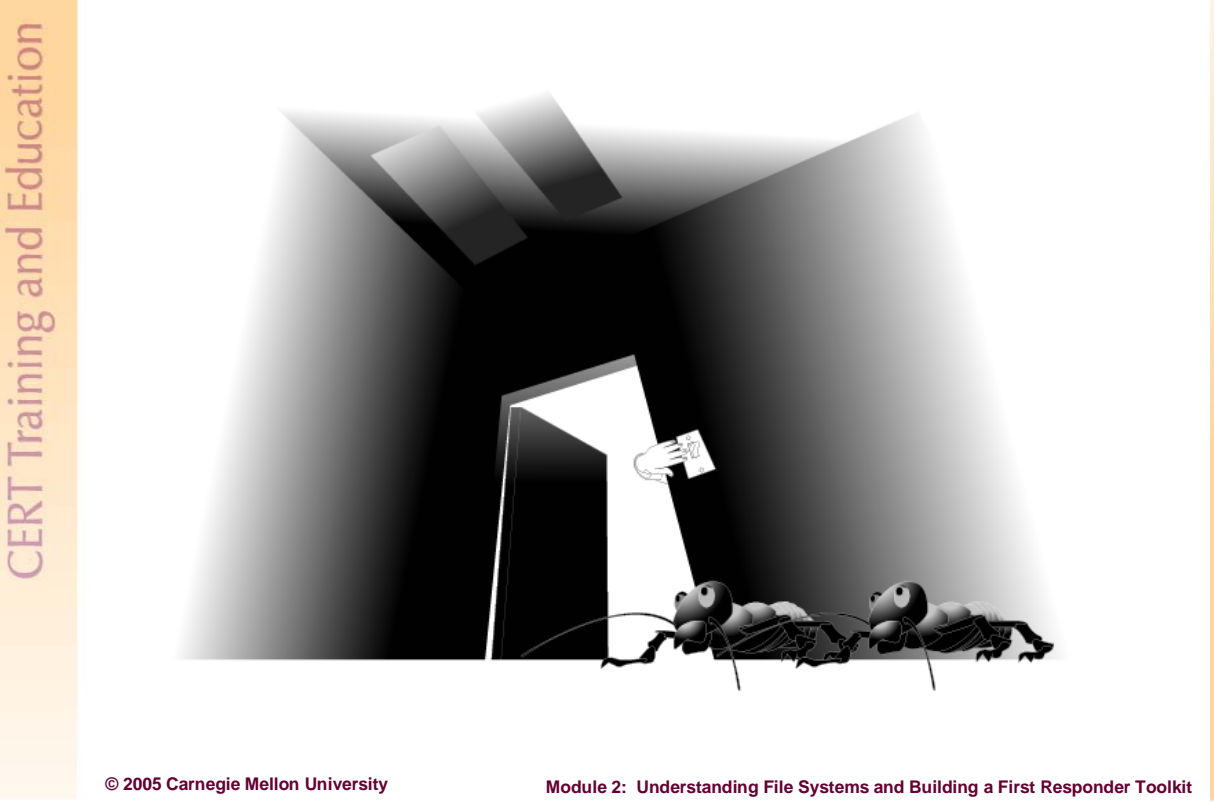

\subsubsection{Boot Sequence}

To ensure that forensic data is not contaminated or altered, a first responder needs to understand how to open and set the suspicious computer's CMOS (complementary metaloxide semiconductor) and BIOS (basic input/output system) to boot from a CDROM. Note the BIOS lives inside the CMOS Chip. The CMOS uses a small battery to store system configuration information, including the date and time, when the power is off. The BIOS contains programs that include the instructions for input and output at the hardware level. If a system has a BIOS password usually this can be reset by removing the CMOS battery.

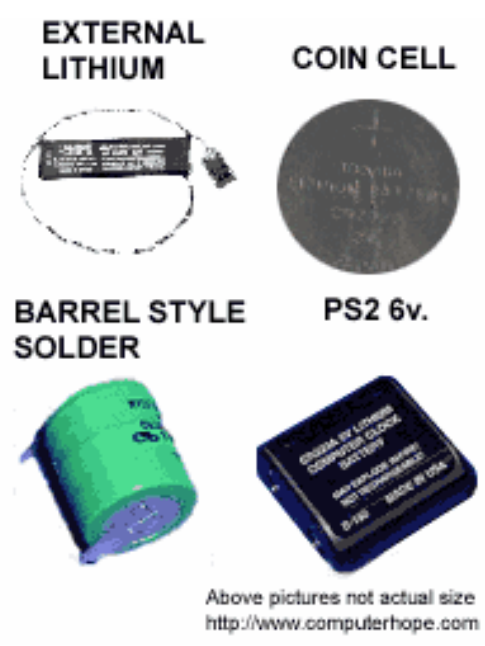

Figure 3: Types of CMOS Batteries 
When a system is restarted, the boot sequence and other start-up activities can modify important forensic data. Therefore, you must enable the BIOS to boot directly from a trusted floppy or CD drive. On most systems, during start-up, the screen usually displays the appropriate key (i.e., Del, Ctrl-Alt-Ins, or a function key) that brings up the BIOS menu. Read the suspicious computer's instruction manual so that you access the BIOS set-up on start-up and prevent unnecessary frustration and possible modification of data. This is an important tip in your pre-incident response preparation: know your systems and maintain a library of manuals and documentation. Many vendors also have CMOS and BIOS information and instruction on their Web site.

For more details, visit the following Web sites:

- http://www.pcguide.com/ref/mbsys/bios/boot.htm

- http://computer.howstuffworks.com/bios.htm

- http://www.computerhope.com/help/cmos.htm 


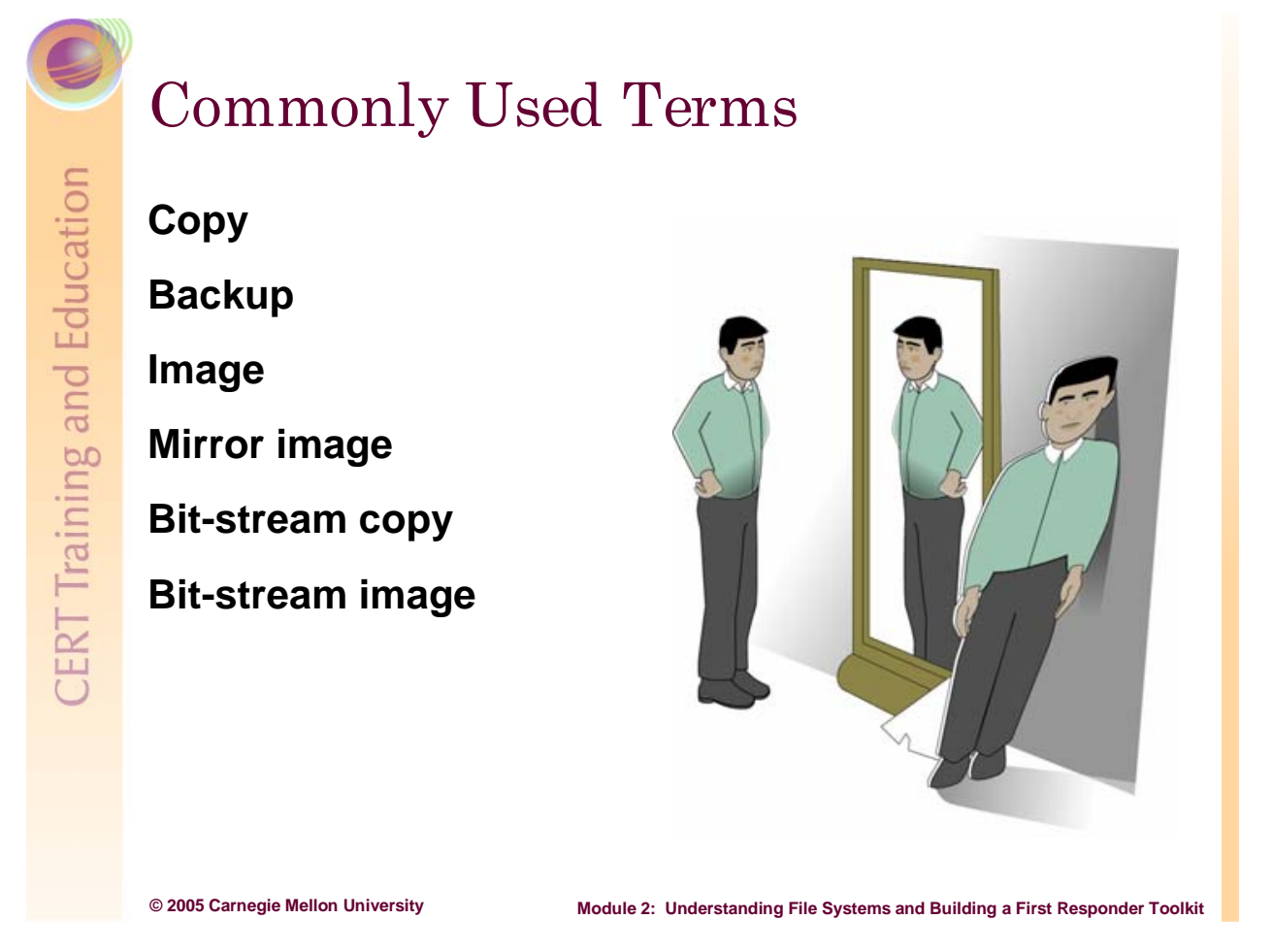

\subsubsection{Commonly Used Terms}

When working with a forensic examiner, clarify the terms "copy" and "image." For example, if another system administrator says "Copy the drive to make an image," he probably needs a backup (just files). But if a forensic examiner asks the same question, he probably needs a duplicate image (every 1 and 0 on the disk) for use as forensic evidence. The following definitions are from New Technologies, Inc [NTI 03]:

Copy: Includes only file information, not slack space or unallocated space

Backup: Files copied for future restoration

Image: A file copy of a complete disk used for duplication or restoration

Duplicate image, bit-stream copy, bit-stream image: Exact replica of all sectors, including every 1 and 0 . This includes slack space, unallocated space, and the swap file. 


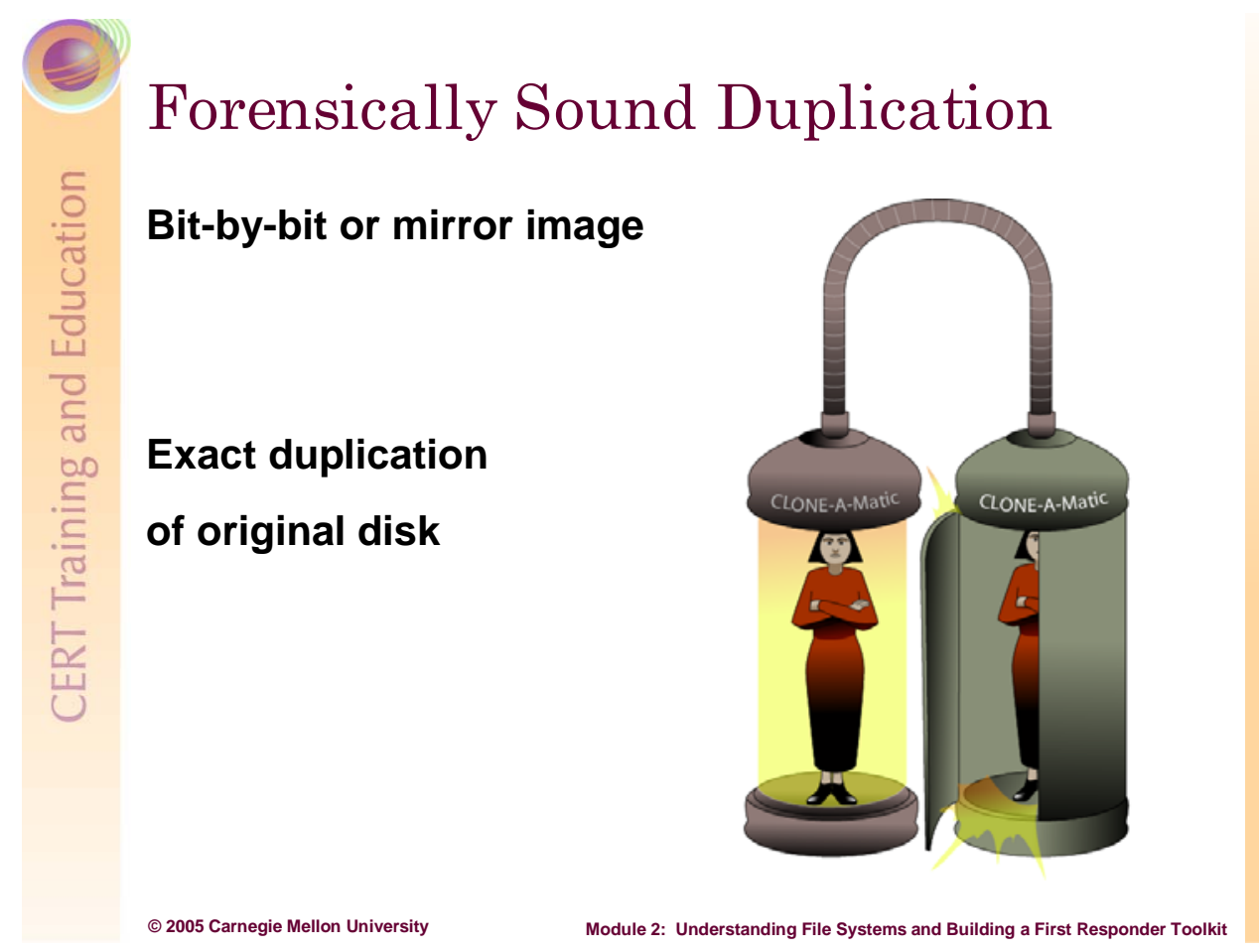

\subsubsection{Forensically Sound Duplication}

Bit-by-bit copy or bit-stream "duplicate” image means capturing every 1 and 0 on the hard drive, including slack space, unallocated space, and the swap file. Understanding bit-by-bit duplication and why it is forensically sound helps you to decide whether to make a copy (captures active files with the OS's copy command) or an image (captures active and latent data) of a drive. 


\section{Duplication Tools}

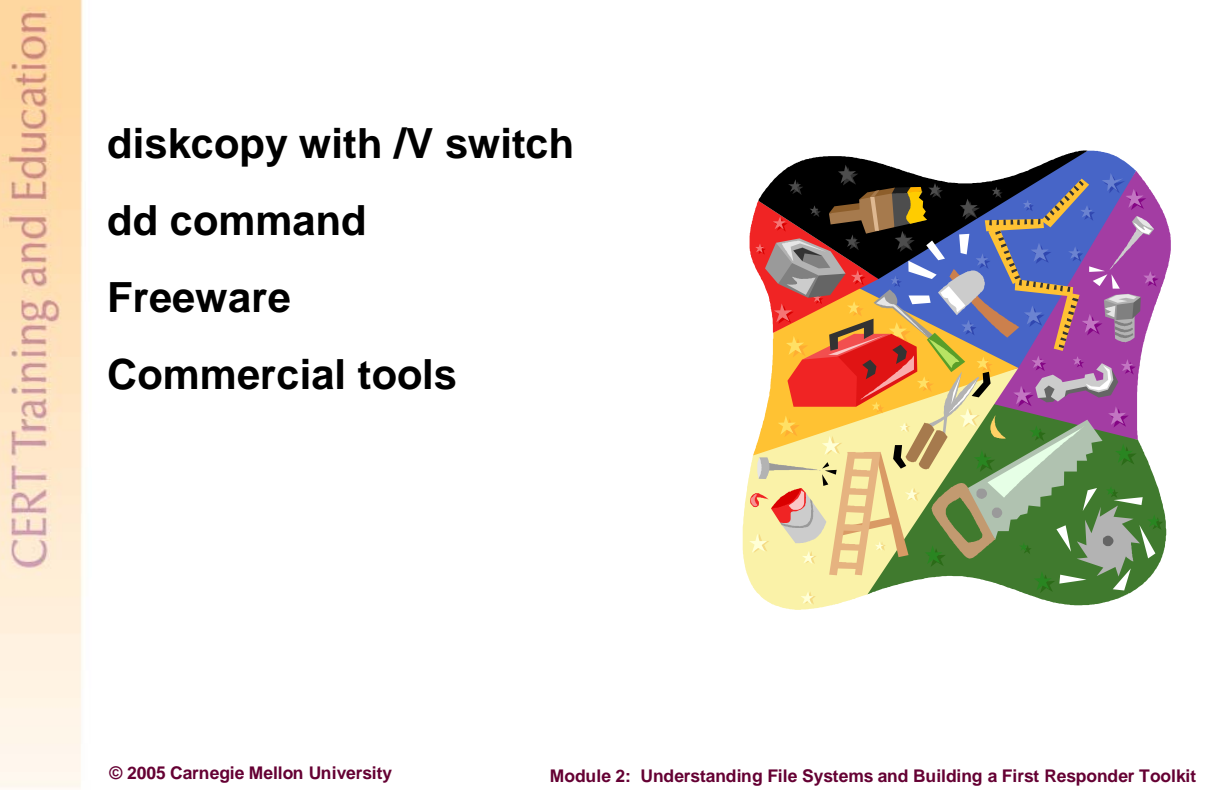

\subsubsection{Duplication Tools}

For a comprehensive list of freeware security and file management tools, visit the following Web sites:

- http://www.cert.org/tech_tips/security_tools.html

- http://www.pcworld.com/downloads/browse/0,cat,1503,sortIdx,1,pg,1,00.asp

More on duplication tools will occur in Module 4. 


\section{Wiping Storage Devices}

\section{Permanent}

Writes over media
with all $1 \mathrm{~s}, 0 \mathrm{~s}$, or
crypto function

Freeware and commercial tools

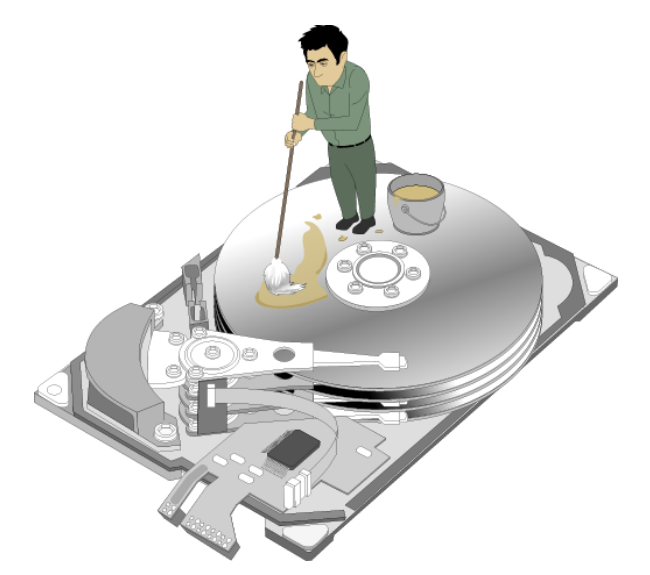

() 2005 Carnegie Mellon University

Module 2: Understanding File Systems and Building a First Responder Toolkit

\subsubsection{Wiping Storage Devices}

An interesting article [MIT 03] demonstrates why disks should be wiped before they are sold, donated, reused, or trashed. Two MIT graduate students looked at 158 used disks and found an assortment of personal and financial information, including over 5,000 credit card numbers.

Recalling from the first part of this module, data that is erased from a hard drive is not necessarily permanently removed. There is a good chance that latent sections remain. The only sure way to be reasonably certain is to wipe the storage device.

For assorted freeware and commercial wipe file/disk products, visit the following Web sites:

- Active@ Kill Disk Hard Drive Eraser v1.1

http://www.pcworld.com/downloads/file_description/0,fid,22920,00.asp

- $\quad$ Sure Delete v5.1

http://www.pcworld.com/downloads/file_description/0,fid,22393,00.asp

- WipeDrive v3.0.2

http://www.pcworld.com/downloads/file_description/0,fid,22961,00.asp

- $\quad$ Eraser v5.7

http://www.pcworld.com/downloads/file_description/0,fid,22963,00.asp

- PGP

http://web.mit.edu/network/pgp.html 


\section{DoD Directive 5220-22M}

DoD "Wipe Out" Operation

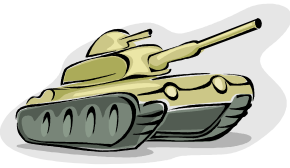

Three iterations completely overwrite a hard drive six times. Each iteration makes two write passes over the entire drive.

The first pass inscribes ones over the drive surface (in hex: 0xFF).

The second pass inscribes zeroes onto the surface (in hex 0x00)

After the third iteration, a seventh pass writes the government-designated code "246" across the drive (in hex 0xF6) which is then followed by an eighth pass that inspects the drive with a read-verify review.

\subsubsection{DoD Directive 5220-22M}

The United States Department of Defense (DoD) issued Directive 5220-22M as part of its National Industrial Security Program. The directive sets security standards for DoD contractors for sanitizing hard drives that contain less than top-secret data.

For more details, visit http://www.dss.mil/isec/nispom.htm. 


\section{Storage Devices -1}

\section{Hard drive}

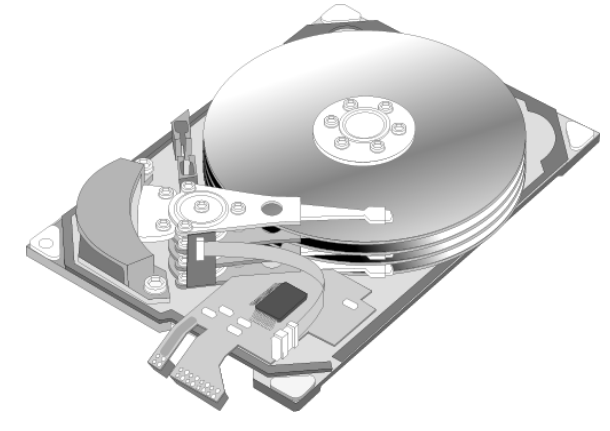

๑) 2005 Carnegie Mellon University

Module 2: Understanding File Systems and Building a First Responder Toolkit

\subsubsection{Hard Drives}

When evidence is expected to be found on a hard drive, these factors need to be addressed:

- Is the drive on the server or a user's computer? The necessary evidence may only be on one partition, not an entire physical drive. Also a user's computer might simply be seized as evidence.

- Is the evidence mission critical or can it be removed? Sometimes it is easier to hand over the original hard drive to the investigators, rather then perform the time consuming duplication process. If the evidence is on a mission critical system it may not be possible unless a hot swappable back-up is available.

- Do you have additional sanitized (wiped) hard drives with the adequate storage capacity? It is good to have prepared hard drives on hand of the proper capacity in case duplication must occur before an investigation team arrives.

- Does the evidence span several physical hard drives? (Raid Array, NTFS Extended Volume) 


\section{Storage Devices -2}

\section{Backup tapes}

Floppy drives

\section{CD/DVD}

USB connected devices

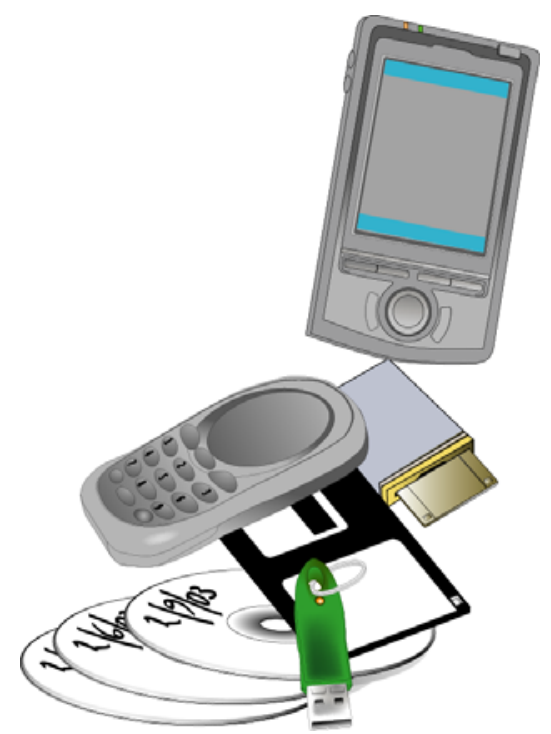

๑) 2005 Carnegie Mellon University

Module 2: Understanding File Systems and Building a First Responder Toolkit

\subsubsection{Other Storage Devices}

There are many types of storage devices, many of which still use the FAT file system. As shown below, storage devices do not have to have the same file system as the OS it interacts with.

Backup tape: host OS

Floppy drive: FAT12

CD/DVD: ISO 9660

Compact Flash, USB thumb drive: usually FAT32 


\section{First Responder Toolkit}

Understand program dependencies

Select tools

Test and verify tools

Understand the benefits to using this methodology

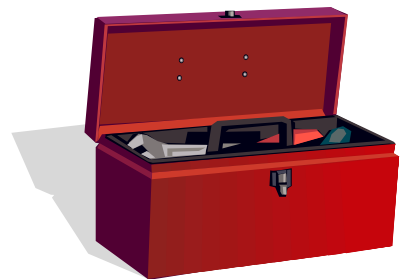

๑) 2005 Carnegie Mellon University

Module 2: Understanding File Systems and Building a First Responder Toolkit

\subsection{First Responder Toolkit}

It is important to understand that network and security professionals should have trusted tool kits prepared. When responding to a system whose trust state cannot be known, it should be considered untrustworthy until proven otherwise.

When a program is executed, it normally uses shared libraries for routine system commands and changes those common files' access times. This is important for forensic examiners as they examine machines to determine when activities took place. It is this issue that prompts the creation of a trusted tool. A trusted tool should have the needed libraries statically compiled when possible.

Also, as a first responder, you'll want to select tools that create output-specific information and then test it to determine system dependencies so that it can be statically compiled (best case) and well documented. 


\section{Statically- vs. Dynamically-Linked Tools}

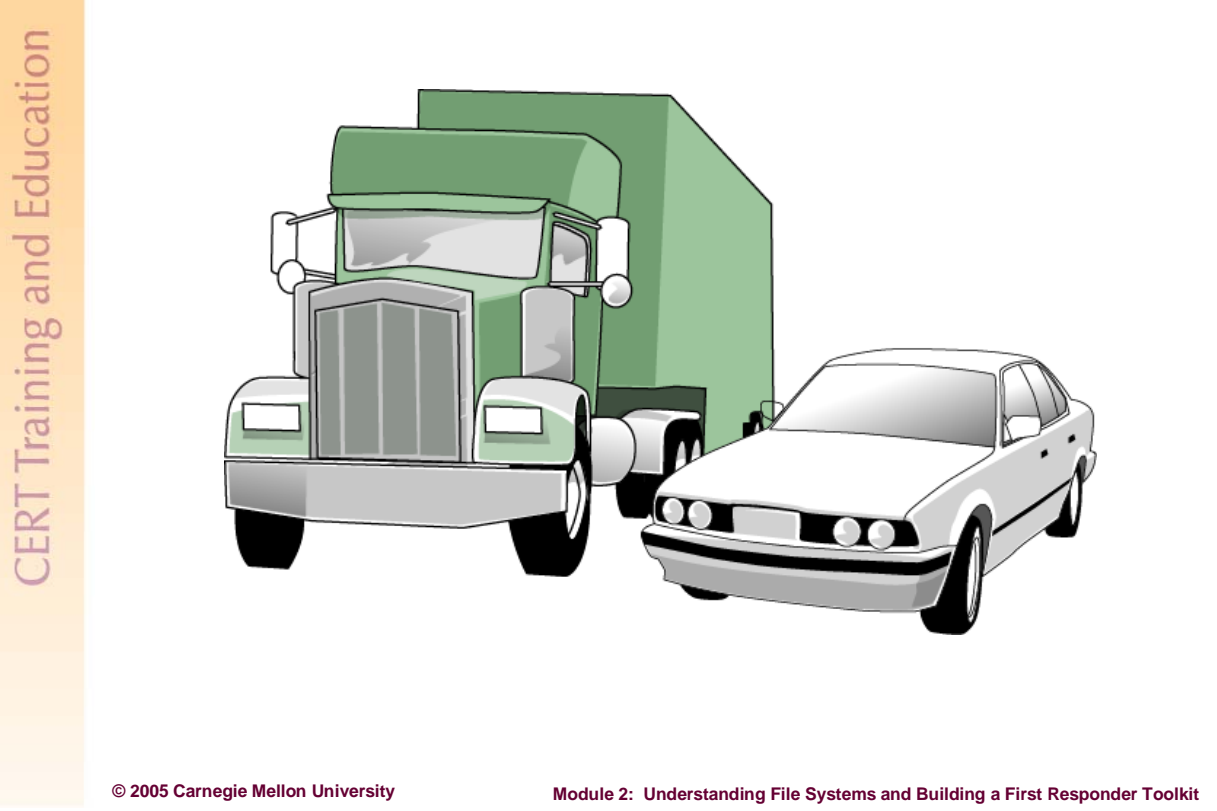

\subsubsection{Statically- vs. Dynamically-Linked Tools}

The significant difference between a statically-linked executable and a dynamically-linked executable is that a statically-linked executable is self-contained: the program contains all the code necessary to successfully run as a standalone program. Statically-linked executables or tools are forensically better because they limit the impact (footprint) on the suspicious computer.

However, a dynamically-linked executable uses loaded libraries within the computer's memory to run. An example of a dynamically-linked executable that could be used by a forensic examiner is the Windows native netstat.exe command. This executable uses multiple dynamic-link libraries (DLLs) in order to properly execute.

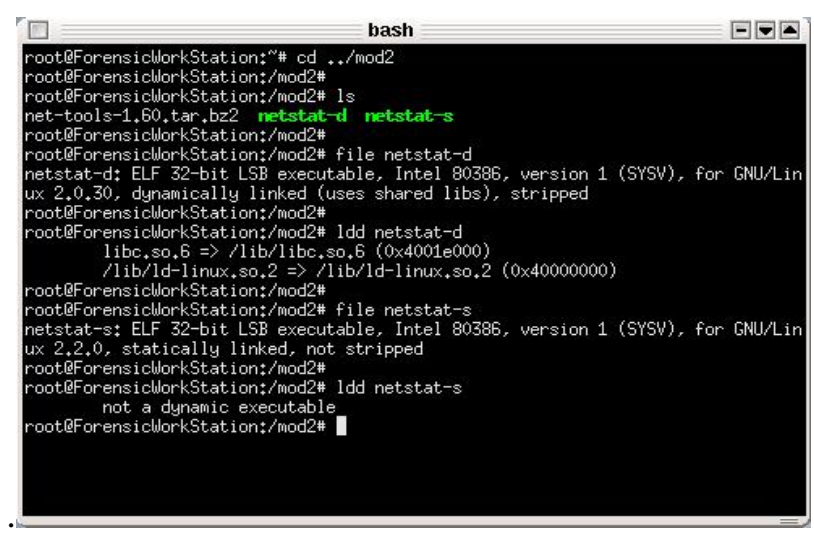

Figure 4: The Idd Command 
In dynamic linking, a link is established between the executable and the required libraries. Within a binary, a table called an import directory or a variable-length array of imports contains the name of each required DLLs. At loadtime, the OS's loader loads the executable and the libraries separately. The loader searches the hard disk for the required libraries (generally located in the Windows System32 folder), loads them into an unpredictable location within memory, and updates the executable with the library's location.

An executable then uses this information to call functions and access data stored in the library. This type of dynamic linking is called loadtime linking and is used by most OSs including Windows and Linux. One disadvantage of dynamic linking is that the executables depend on the separately stored libraries in order to function properly. If the library is deleted, moved, renamed, or replaced with an incompatible or malicious version, the executable could malfunction and cause unreliable and false output [Wikipedia 04].

This further explains why including the necessary DLLs for an executable on a separate disk will not ensure that the binary will use the DLLs on the separate disk. It also explains why the first responder should never trust or use the native commands on the suspicious computer for collecting volatile data. 


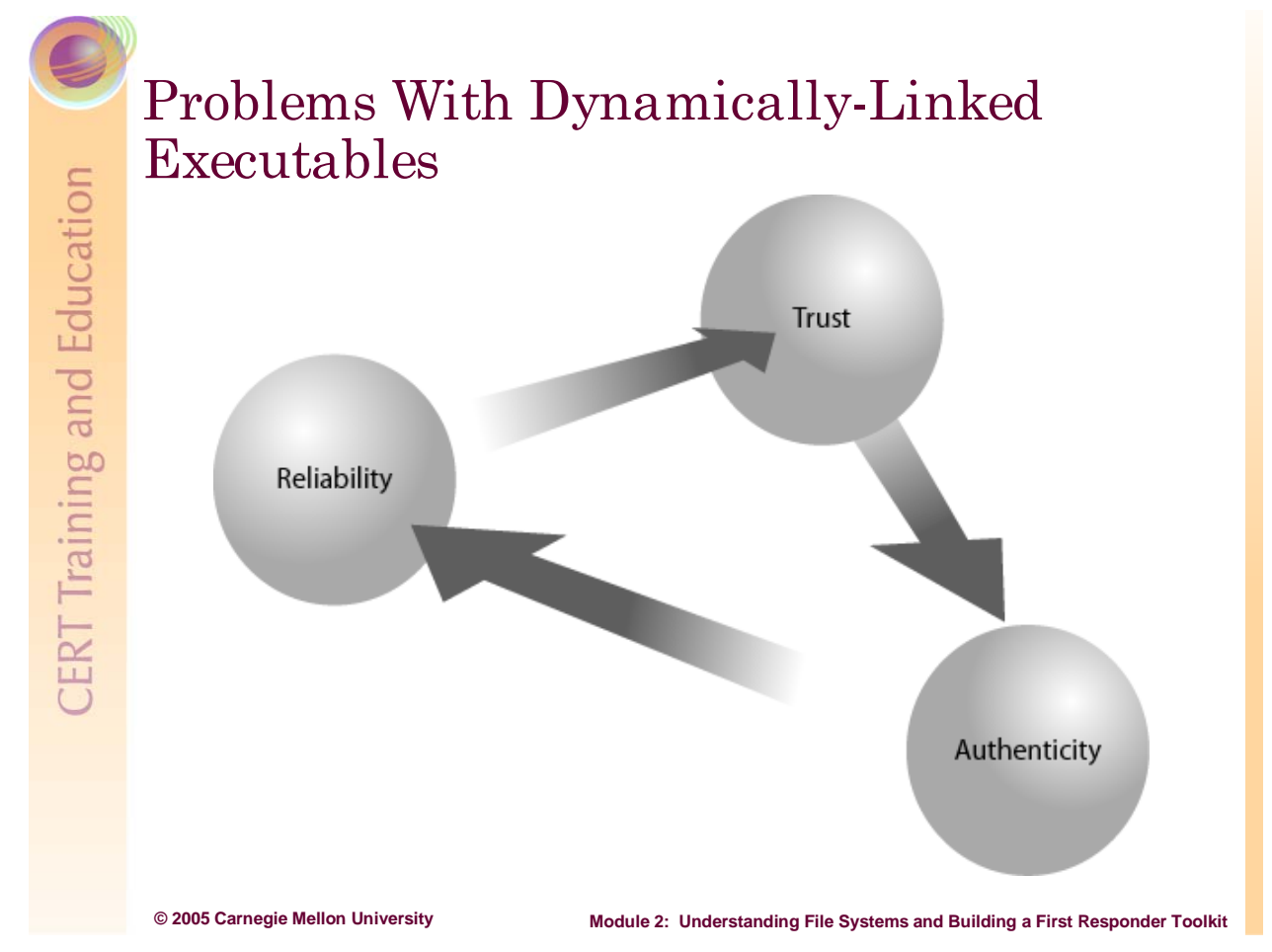

\subsubsection{Problems with Dynamically-Linked Executables}

One issue in creating a first responder toolkit is the problem with forensic tool dependencies and DLLs in Windows. Because Windows code is closed and not publicly available it is not possible to statically compile Windows native executables. Therefore, at a minimum it is important to define what DLLs and files are affected when executing a Windows program. With this information, it is possible to at least note what impact (or footprint) the program will have on the system.

To determine which files or shared libraries each forensic tool accesses, use the Filemon utility. Once the dependencies have been identified, include the list of files on the response disk so that in the event that it becomes necessary, this list can be provided and be used to identify what changes to the system were made by the first responder.

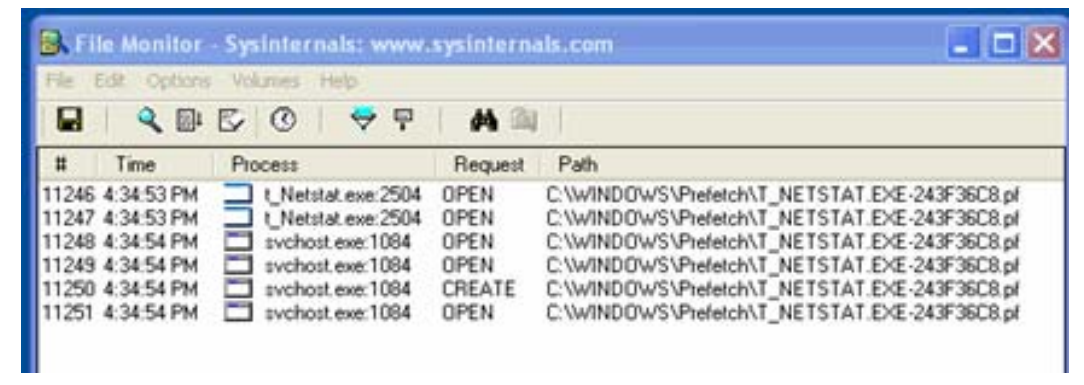

Figure 5: Using Filemon to Identify Dependencies 
The issue with DLLs is not as pressing in the UNIX world. Since most of the operating systems are open source it is possible to statically build binaries that will not rely on shared libraries. This is the preferred type of tool because its output is more trustworthy. 


\section{Methodology for Creating a First Responder Toolkit}

\section{Create the forensic tool testbed}

2. Document the testbed

\section{Document and set up forensic tools}

\section{Test the tools}

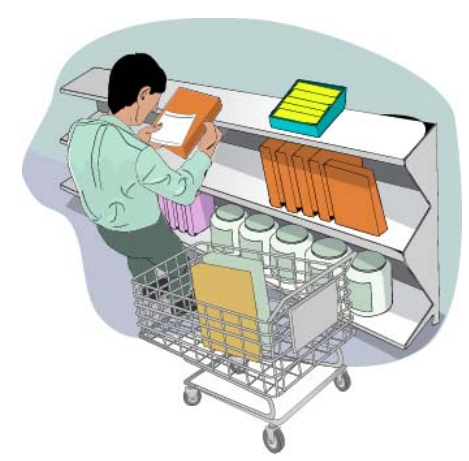

Module 2: Understanding File Systems and Building a First Responder Toolkit

\subsubsection{Methodology for a Creating First Responder Toolkit}

Before a security incident occurs and before any evidence collection is done, the first responder must develop a toolkit. In most computer crime cases, the first responder will be the system administrator or a member of law enforcement. In either case, being prepared beforehand is crucial; you do not want to waste time putting together a set of tools at the last minute that you think might do the job. As you create your toolkit, you will become more familiar with forensic collection tools and native commands and their proposed functionality.

A working knowledge of the tools in your toolkit will aid your collection efforts and help you understand your collection limitations and capabilities.

The methodology for creating a first responder toolkit includes of the following four procedures:

1. create a forensic tool testbed

2. document the testbed

3. document and set up the forensic tools

4. test the tools

As a first responder, you should follow this methodology to ensure the integrity and reliability of each collection tool, command, or application you use in the field. It provides guidelines and reproducible procedures for future toolkit verification. 


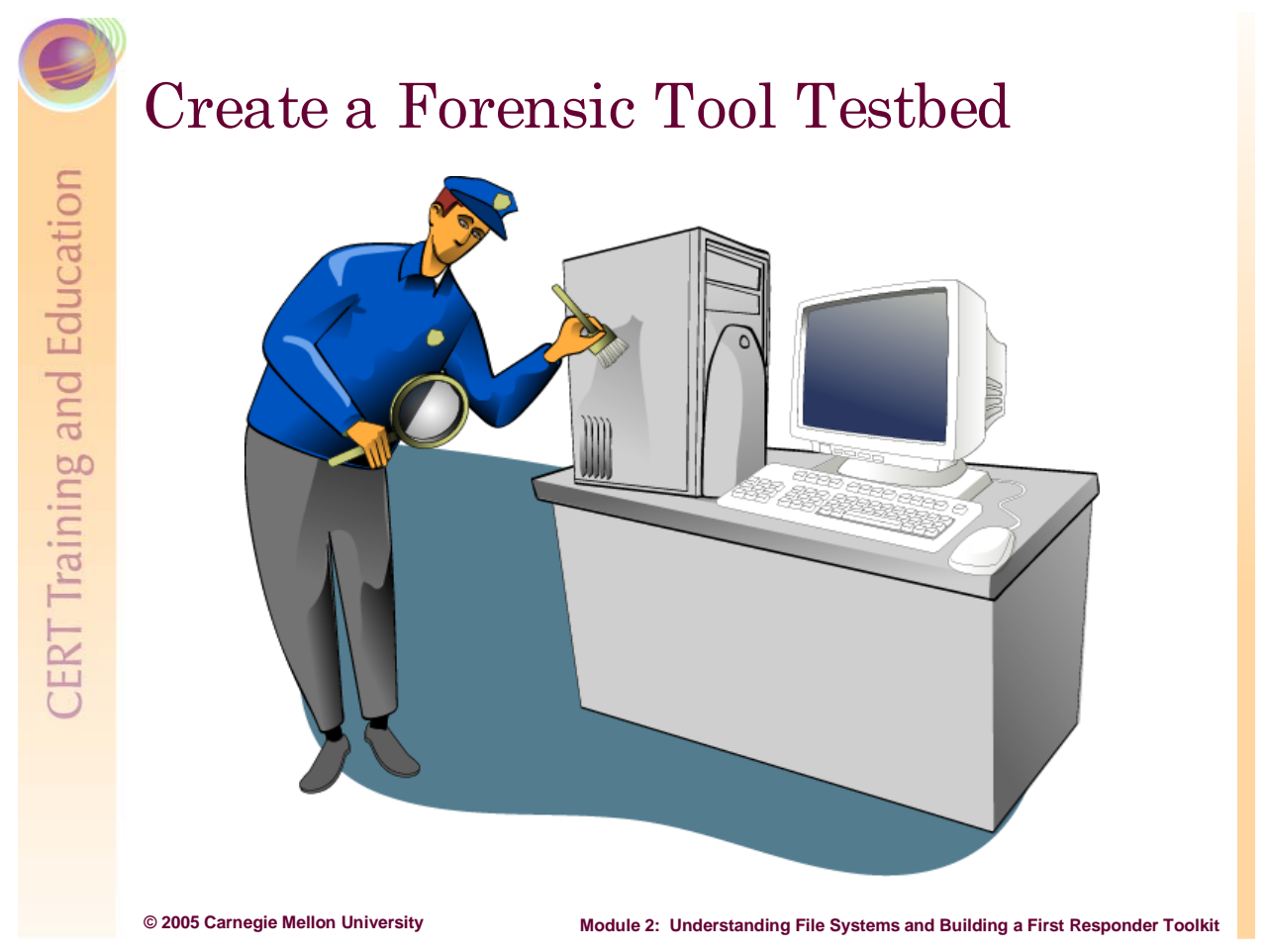

\subsubsection{Create a Forensic Tool Testbed}

The first step in creating a first responder toolkit is to create a trusted forensic computer or testbed. This testbed will be used to test the execution and functionality of each forensic collection tool you consider using. But before any tool is tested, ensure that the forensic tool testbed is a trusted resource. It should be a fresh install, free of artifacts from any previous use that could compromise its integrity and reliability. The testbed should be comparable to the systems that you are responding to.

\section{Identify the OS Type}

Choose an OS type. If the enterprise you respond to is primarily Windows based, it makes sense to use a Windows forensic tool testbed. The same holds true for Linux. If the enterprise has Windows and Linux machines, you may want to create two different testbed machines, one for each type of OS you may be dealing with. However, VMware allows you to install multiple OSs on a single machine.

\section{Sanitize the Testbed}

Completely sanitize the machine so that you can start with a fresh slate. This includes a complete (forensic) wipe of the hard drive(s) to remove any data or artifacts left from the machine's previous use. The following freeware is available.

- BCWipe (Windows)

http://www.jetico.com/index.htm\#/bcwipe3.htm 
- Wipe (Linux)

http://wipe.sourceforge.net/

\section{Install Software}

Install the OS and necessary software applications from trusted resources (i.e., designated CD-ROMs for each application and OS). If you have downloaded open source OSs (e.g., Linux variants), then you must verify the hashes before installing the OS. Typically, the testbed system has a minimal set of software applications installed, but it's better for the tool testbed system to replicate a critical system within your IT and network infrastructure so that you know how to respond to machines in that similar state or that have critical services running.

\section{Update and Patch}

Bring the system up to date. This is just using best practices and you should treat this testbed machine like any other machine in your IT and network infrastructure. Any necessary patches or hot fixes should be applied to the machine. Again these updates and patches must be applied offline and from a designated disk with known hashes. This 'patch CD' should become a documented item in your tool kit.

\section{Hash the DLLs}

For Windows testbed machines, perform a cryptographic hash of the installed DLLs on the system while off-line. This produces checksums for the DLLs at their initial known safe state. After you run and test your forensic tools, you can rehash and compare before and after checksums to determine if any DLLs have been modified or accessed.

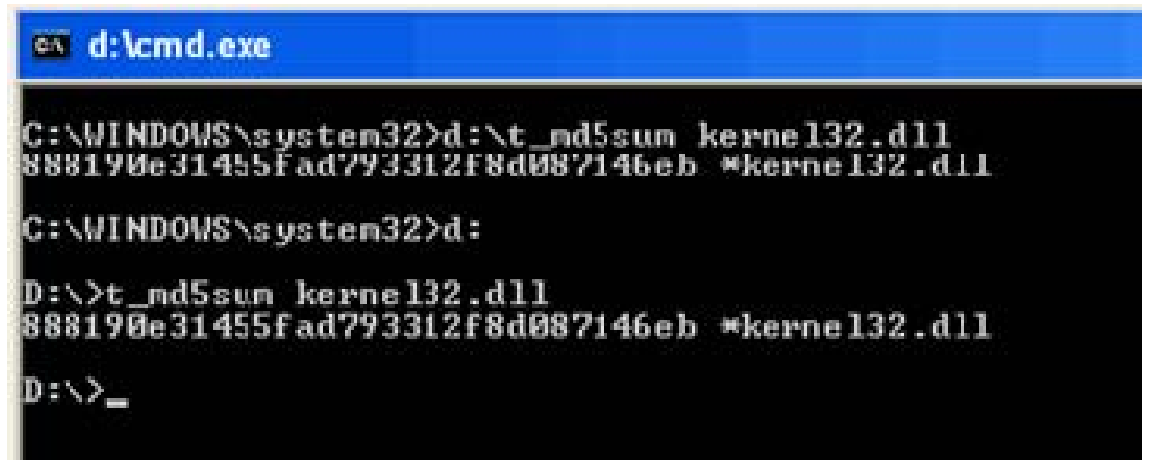

Figure 6: Performing a Cryptographic Hash of Installed DLLS

For Linux testbed machines, you may want to identify all shared libraries on the system and compute a hash of all the shared libraries. Again, rehash the shared libraries after running your tools and then compare checksums. 


\section{Install a File Integrity Monitor}

Using a file integrity monitor on the testbed system is a best practice. File integrity monitors like Tripwire or LANguard System Integrity Monitor (SIM) should be installed on the forensic testbed to monitor the integrity of the testbed computer's file system. Later, during tool tests, Tripwire or LANguard can identify changes to MAC times.

Another file system utility called Filemon monitors which files each forensic tool uses or references.

For more details, visit the following Web sites:

- http://www.sysinternals.com/ntw2k/utilities.shtml

- http://www.tripwire.org/downloads/index.php

- http://www.gfi.com/languard/ 


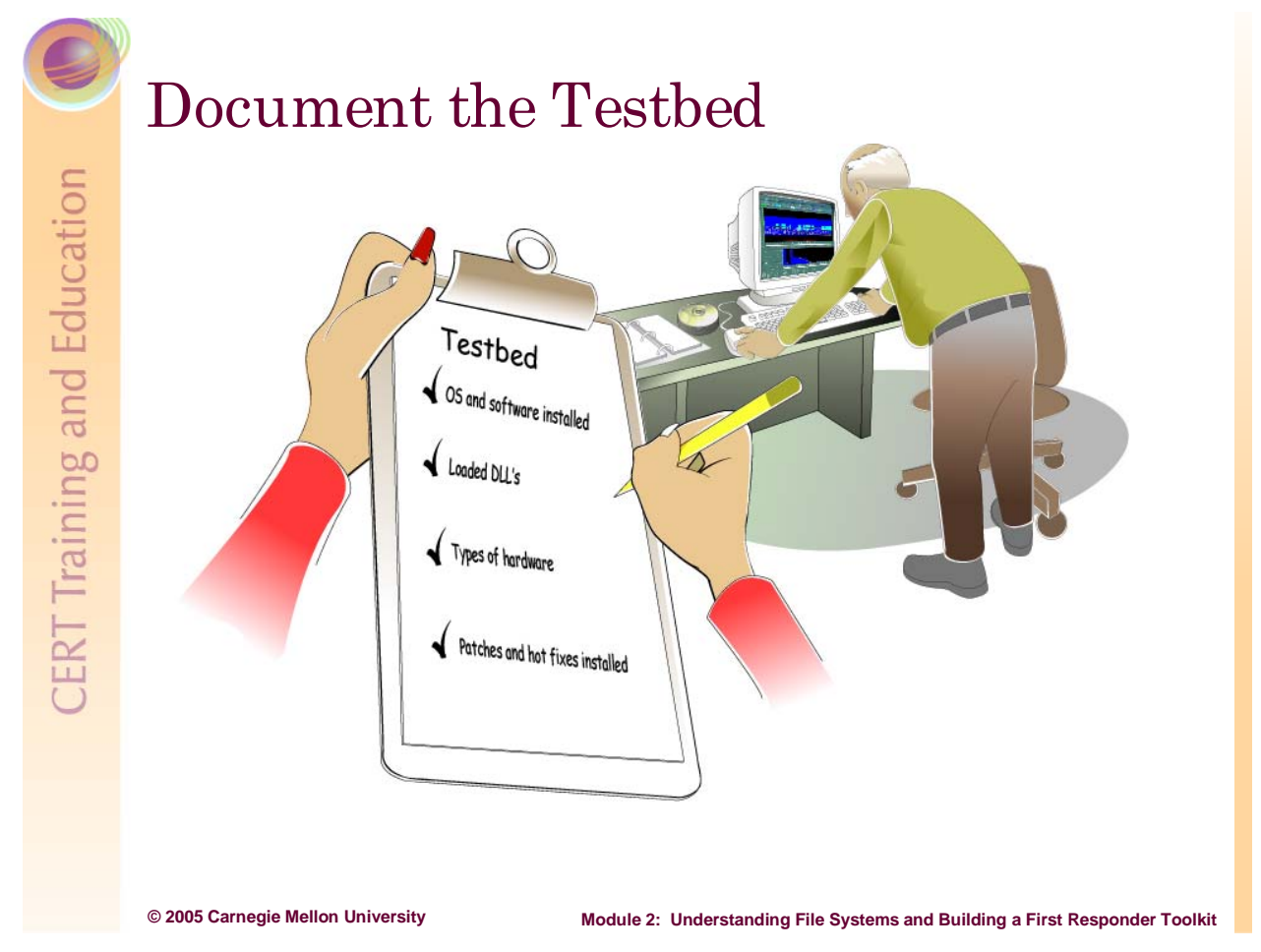

\subsubsection{Document the Testbed}

Documenting the testbed computer(s) is as important as creating them. If you respond to a security incident and the integrity and reliability of your tools and collection procedures are questioned, an independent expert could use your documentation to easily reproduce the situation.

The forensic tool testbed profile includes the following information:

- type of OS

- version of OS (i.e., kernel)

- number and types of applications

- number and types of patches and hot fixes

- types of hardware installed

- MD5 hash of DLLs/shared libraries 


\title{
Document Forensic Tool Setup
}

\author{
Document \\ Acquisition \\ Description
}

Functionality

Dependencies and system affects

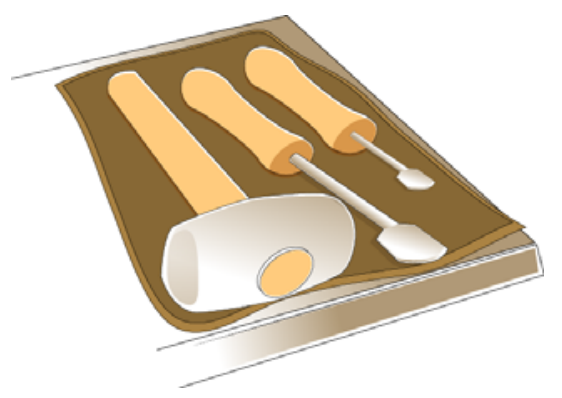

Create a response disk

\subsubsection{Document and Set Up the Forensic Tools}

Creating a profile of forensic tools is as important as creating a profile for the testbed. For each tool that you identify to test, document the following characteristics: acquisition, description, functionality, and dependencies and system affects. By creating a profile of each tool you plan to test, you become familiar with each tool and understand how you can use or tailor the tool to your incident response needs.

\section{Document the Tools}

Acquisition: Document how and why you acquired each tool. Identify whether you acquired the tool by downloading, developing, or purchasing it. Document how you verified the integrity of the tool while acquiring it (i.e., verified the MD5 hash for downloaded tools, or testing/validation done for developed/purchased tools).

Description: Document details including — but not limited to-what OS the tool is compatible with, how the tool operates, and how you use the tool (command line vs. GUI).

Functionality: Document as many of the following as apply: the expected generated output; volatile information that can be collected; command-line switches or syntax.

Dependencies and System Affects: Tool dependencies and system affects are probably the two most important tool characteristics to identify and document. You can discover a preliminary set of tool dependencies and system affects by reading the tool's description or read me file, but you will not discover the complete set until you test the tool. 
Tool dependencies include required access levels and the use of shared libraries. They are crucial and could limit your forensic collection efforts. Therefore, if you do not have administrative or root credentials, you probably will not want to use tools that require that access.

As you document file dependencies like shared libraries, DLLs, and registry keys, you will recognize what kind of footprint the tool leaves on a system and can use that information as follows.

- For Linux systems, you can statically recompile the command so that it will no longer reference the system.

- For Windows systems, you cannot recompile but you can rank tools based on which has the fewest dependencies and system affects.

\section{Create a Response Disk}

Tools should reside on a separate storage media device like a floppy disk, thumb drive, or CD-ROM. (For simplicity, we assume disk and refer to the "response disk.") Which one you choose depends upon space requirements, how many tools you have, and what you think you will have access to when responding to an incident. Systems will generally have a USB port, CD-ROM drive, or floppy drive, but it is a good idea to have your forensic tools on multiple storage devices.

Any other items that are required for the tool to run (i.e., other executables, DLLs, and other files) should be copied onto the response disk. Shared libraries required for Linux commands should be copied to the response disk and the executable associated with the command should be statically compiled to ensure that the command does not reference the system as it is executed. The first responder identifies these required files and shared libraries during tool testing, the final step in this first responder toolkit methodology.

Determine how you will save the forensic tool output. You can save the output to the response disk or to another system on the network. The output should never be saved to the live suspicious computer.

Label your response disk properly with the following minimum information:

- $\quad$ time and date

- name of person who created and verified the response disk

- if the response disk contains any output files or collected evidence

The National Institute of Justice (NIJ) provides guidelines for documenting computer evidence [NIJ 04]. 


\section{Test the Tools}

\section{Install tool analysis utilities}

Perform a static analysis

Perform a dynamic analysis

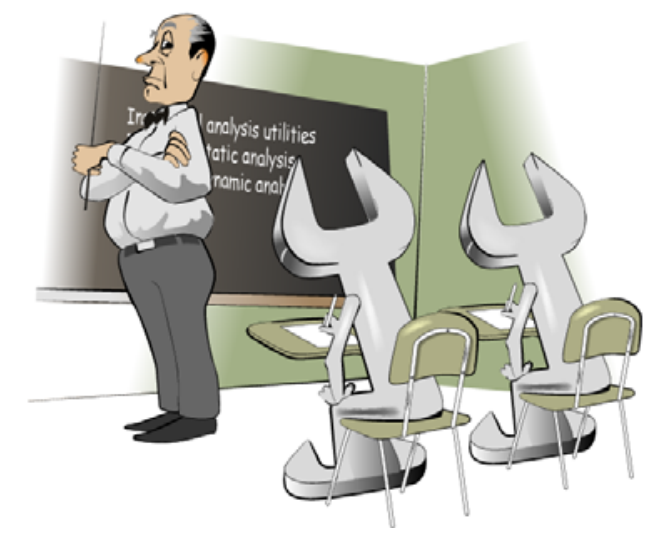

\subsubsection{Test the Tools}

Now that you have identified and profiled forensic tools, you are ready to test each tool on the forensic testbed. During testing, the first responder can examine tool performance, functionality, and tool generated output and-most importantly-determine what affects the tool may have on a typical system.

Utilities like Regmon, Filemon, ListDLLs, Dependency, LANguard or Tripwire determine each tool's impact on the system. They allow the first responder to monitor and catch changes or references to the forensic testbed system caused by the forensic tools and provide output that can be used to document the tools.

\section{Install Tool Analysis Utilities}

Acquire, unzip, and install the following utilities on the testbed system.

\section{Filemon (Windows, Linux): Open and configure Filemon.}

Filemon monitors and displays file system activity on a system in real time. Its advanced capabilities make it a powerful tool for exploring the way Windows works, seeing how applications use the files and DLLs, or tracking down problems in system or application file configurations. Filemon's time-stamping feature shows you precisely when every open, read, write, or delete occurs, and its status column displays the outcome. It begins monitoring when you start it, and its output window can be saved to a file for offline viewing. It has full search capability that can be filtered if you experience information overload [Russinovich 04d]. 
Regmon (Windows): Open and configure Regmon.

Regmon monitors the registry and shows you —in real time-which applications are accessing your registry, which keys the tools are accessing, and the registry data that they are reading and writing. With static registry tools, you might be able to see which registry values and keys changed. With Regmon, you see which values and keys are changed and how [Russinovich 00].

\begin{tabular}{|c|c|c|c|c|c|c|}
\hline \multirow{2}{*}{\multicolumn{7}{|c|}{ 64. Registry Monito: - Sysinternals: www.sysinternats.com }} \\
\hline \multicolumn{3}{|c|}{ File Edit Options Help } & & & & \\
\hline \multicolumn{7}{|l|}{ E } \\
\hline$\pi$ & Tine & Pro: & cents & Requet & Pat & 스 \\
\hline \multicolumn{2}{|c|}{ अ19E 69501921529 } & $\Rightarrow$ & ENeratoteser2504 & Ebrexe: & 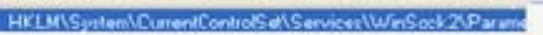 & \\
\hline \multicolumn{2}{|c|}{31197696.01927647} & $\bar{\Xi}$ & Nelstal exe 2504 & CloseKer. & HKLMISytemiCurentContiols el ServicestWinSock 2 Paryme & \\
\hline \multicolumn{2}{|c|}{31198696.01941364} & ב & Netstat ene 2504 & Operkes & 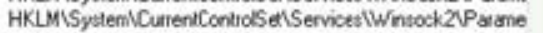 & \\
\hline \multirow{2}{*}{\multicolumn{2}{|c|}{$\begin{array}{l}31194 \mathrm{k9k} \text { m145727 } \\
31200 \quad 696.01551505\end{array}$}} & コ & Detedit now 2054 & nuapviala & 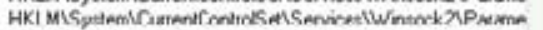 & \\
\hline & & コ & Netstat ene 2504 & Closekes & 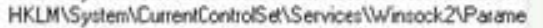 & \\
\hline \multicolumn{2}{|c|}{$31201696.01 \$ 81509$} & $\overline{0}$ & Netstatese 2504 & Operkes & HKLMISystemi CurrentControlS exlContholSestion Manager & \\
\hline \multirow{2}{*}{\multicolumn{2}{|c|}{$\begin{array}{l}31202656.01987878 \\
312006966.01994511\end{array}$}} & 马 & UNetstat.ene 2504 & Queryivae & 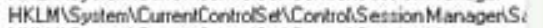 & \\
\hline & & コ & Netstat ene 2504 & Closekos & HKLMISystemVCurrentControlS atControliSestion Manager & \\
\hline \multirow{2}{*}{\multicolumn{2}{|c|}{31204696.02162565}} & 己 & Netstatene 2504 & Operkes & HKLMISoftwse WictonoftWWindowa NTV CurterkNersionNimag & \\
\hline & & & t Netstat esen 2504 & Operker & 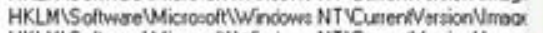 & \\
\hline \multicolumn{2}{|c|}{$3120 \mathrm{~B} 696.02199386$} & 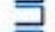 & tinetstat ese 2504 & Operkes & HKLMIS oltwse Micsosoft'Windows NT CureriNersion Nmag. & \\
\hline \multicolumn{2}{|c|}{31207696.03474157} & $\sqsupset$ & Wetstat ene 2504 & Operkes & 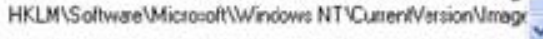 & \\
\hline
\end{tabular}

Figure 7: A Regmon Listing

\section{ListDLLs (Windows): Run ListDDLs.}

ListDLLs shows you the full path names of loaded DLL modules. In addition, ListDLLs will flag loaded DLLs that have different version numbers than their corresponding on-disk files (which occurs when the file is updated after a program loads the DLL), and can tell you which DLLs were relocated because they are not loaded at their base address [Russinovich 00].

Dependency (Windows): Run depends.exe.

Dependency Walker is a free utility that builds a hierarchical tree diagram of all dependent modules. For each module, it lists exported functions and functions called by other modules. Another view displays the minimum set of required files and details about each file.

For more details, visit http://www.dependencywalker.com.

LANguard (Windows) and Tripwire (Linux): Set up, configure, and run Tripwire or LANguard SIM.

LANguard and Tripwire monitor file system integrity. These utilities will prove to be useful in seeing if the tools change the MAC times of any files in the file system. If the tool that you are testing does in fact touch the file system during your testing it is extremely important to document this and see if the changes were significant or malicious. 
Strace.exe (Windows): Strace executes a program, and optionally the children of the program, stdout. Use the -o option to send output to a file. Use the -w option to start a strace session in a new window. This is useful for sessions that take a long time to complete. It is meant to be used like the strace (or truss) on Linux.

Strace for Windows systems is still in a Beta status and should be used with caution.

For more details, visit http://www.bindview.com/Support/RAZOR/Utilities/Windows/.

Exetype.exe (Windows, Linux): Exetype is a command-line utility available in the Windows Resource Kit. It is used to find the executable type and determine the OS and processor required to run the program file. It is useful when dealing with an unknown program.

\section{Perform a Static Analysis of the Tools}

Static and dynamic analyses allow you to disprove an initial assumption that each forensic tool is malicious. In a static analysis, you review each tool's documentation and file types, perform string searches, and view binary data (the executable) using a hex editor. In the process, a better understanding of the functionality, trustworthiness, and reliability of each tool is gained.

\section{Tool Documentation}

Review the tool's documentation to determine its functionality, supporting commands, and proposed system affects.

\section{Determine the File Type}

Determine the file type using the Linux file command or the Windows Resource Kit exetype command. This verifies that the correct file type is reflected in the downloaded tool's filename extension.

\section{Display Legible Strings}

Display legible strings, such as ASCII and Unicode strings, within the binary using the Linux or Windows command strings. Look at the keywords, command-line arguments, and variables to understand the tool's purpose [Mandia 01].

The strings command in Linux displays all humanly legible strings longer than four characters. If the programmer was verbose, these strings can reveal function names, variable names, defined strings, and — if you are lucky—complete pathnames of the source code used to build the executable.

Always use the -a flag, as shown below, to process the entire file.

$$
\text { strings -a }<\text { filename }>\text { | less }
$$




\section{View Binary Data}

View binary data to uncover abnormal function names. The rogue code's names may provide clues about the function's purpose.

For Linux systems, use the GNU debugger $g b d$ with info variables as shown below.

$$
\text { gbd }<\text { filename }>\text { info variable }
$$

For Windows systems, use any freeware hex editor.

\section{Perform a Dynamic Analysis of the Tools}

In a dynamic analysis, you use traces to determine file access and dependencies. In the process, you can determine the total system impact of each tool.

Document and save all results.

\section{$\underline{\text { Kernel Access }}$}

Strace is a Linux utility that wiretaps the system and reports every access of kernel resources. Whenever an application or tool writes to the screen, performs IO with network, disk, or other devices, or allocates memory, strace tracks it. Consider performing string searches on the outputted file, which can be difficult to read [Mandia 01].

Exetype is a Windows Resource Kit utility and a Linux command. Exetype is a power tool for extracting information about a running executable.

\section{$\underline{\text { File System and Registry Key References }}$}

Use Filemon and Regmon to trace each tool's file system and registry key references during execution. Before you begin, set the utility's filters to look for the filename of the forensic tool's executable only so that references by all other tools and application are ignored.

Review the Filemon and Regmon filter displays to see if your tool references any part of the file system outside of its own location (i.e., CD-ROM or thumb drive), and then document the output. For outside references, document the target and the time it was referenced.

\section{DLL/Shared Library Dependencies}

The following utilities identify which files each forensic file references, which allows you to rank each tool's impact on a system and understand the expected side affects of each tool.

Windows: Use ListDLLs or Dependency to determine DLL dependencies.

For Dependency, use the Open button to navigate to and open your tool. Dependency then displays all DLLs required for the tool to run. 
For ListDLLs, run your forensic tool first and then run ListDLLs to display the loaded DLLs.

Linux: Use the ldd command to determine shared library dependencies. The ldd command prints the shared libraries required by each program or command specified on the command line.

\section{Create a First Responder Toolkit Logbook}

After testing, create a folder or file that includes the following for each forensic tool:

- the profile

- documentation

- $\quad$ static analysis results

- dynamic analysis results

This logbook should be kept handy. During the actual data collection described in Modules 3 and 4, you will refer to this book to determine which tools best fit your system and situation. 


\section{Benefits of Proper Tool Testing}

Incident

\section{Explaining to law enforcement forensic investigations}

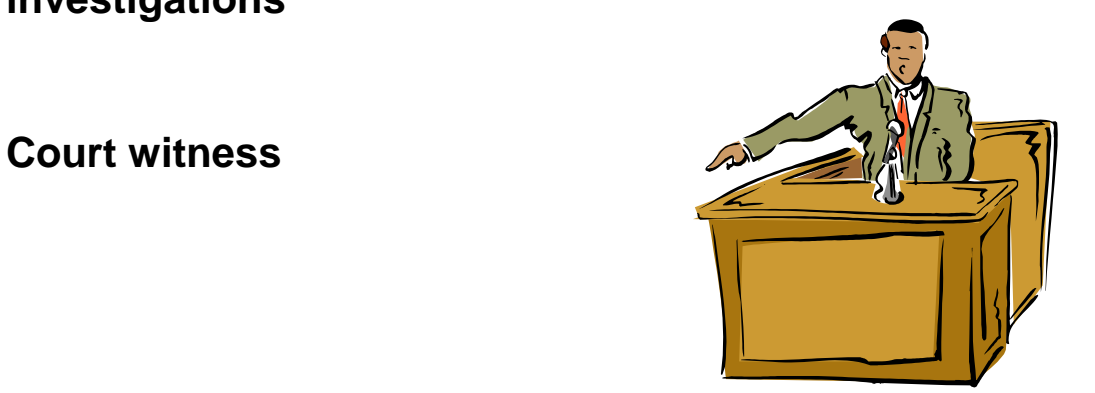

\subsubsection{Benefits of Proper Tool Testing}

If you follow these best practices for testing tools before you use them, you can significantly reduce the time it takes to respond to a security incident while establishing the reliability of each tool's output for use in a forensic investigation or court of law. 


\title{
NIST Methodology
}

\author{
NIST: National Institute of Standards and Technology, \\ Information Technology Laboratory, Computer \\ Forensic Tool Testing Program
}

The Computer Forensics Tools Verification project provides a measure of assurance that the tools used in the investigations of computer-related crimes produce valid results. It also supports other projects in the National Institute of Justice's overall computer forensics research program, such as the National Software Reference Library (NSRL).

http://www.cftt.nist.gov/

\subsubsection{NIST Methodology}

Before we developed our methodology for creating a first responder toolkit, we examined the National Institute of Standards and Technology (NIST) Computer Forensics Tool Testing Program [NIST 03]. The following is an excerpt.

\section{CFTT Methodology Overview}

The testing methodology developed by NIST is functionality driven. The activities of forensic investigations are separated into discrete functions or categories, such as hard disk write protection, disk imaging, string searching, etc. A test methodology is then developed for each category. Currently we have developed a methodology for disk imaging tools and are developing a methodology for software hard disk write blocking tools. Deleted file recovery tools will be the next category for development of a test methodology.

The CFTT testing process is directed by a steering committee composed of representatives of the law enforcement community. Included are the FBI, DoD, NIJ (representing state and local agencies), NIST/OLES and other agencies. Currently the steering selects tool categories for investigation and tools within a category for actual testing by CFTT staff. A vendor may request testing of a tool, however the steering committee makes the decision about which tools to test.

Under the disk imaging category the tools selected initially for testing were: Linux dd, and SafeBack. The RCMP hdl was selected for the hard disk write block category. Final test reports are posted to a Web site maintained by NIJ. 


\section{Specification development process}

After a tool category and at least one tool is selected by the steering committee the development process is as follows:

1. NIST and law enforcement staff develops a requirements, assertions and test cases document (called the tool category specification).

2. The tool category specification is posted to the Web for peer review by members of the computer forensics community and for public comment by other interested parties.

3. Relevant comments and feedback are incorporated into the specification.

4. A test environment is designed for the tool category.

\section{Tool test process}

After a category specification has been developed and a tool selected, the test process is as follows:

1. NIST acquires the tool to be tested.

2. NIST reviews the tool documentation.

3. NIST selects relevant test cases depending on features supported by the tool.

4. NIST develops test strategy.

5. NIST executes tests.

6. NIST produces test report.

7. Steering Committee reviews test report.

8. Vendor reviews test report.

9. NIST posts support software to Web.

10. NIJ posts test report to Web. 


\section{Summary}

Importance of understanding

physical hard drive

Different file systems

Duplicating terms

Different media

Wiping data

First responder toolkits

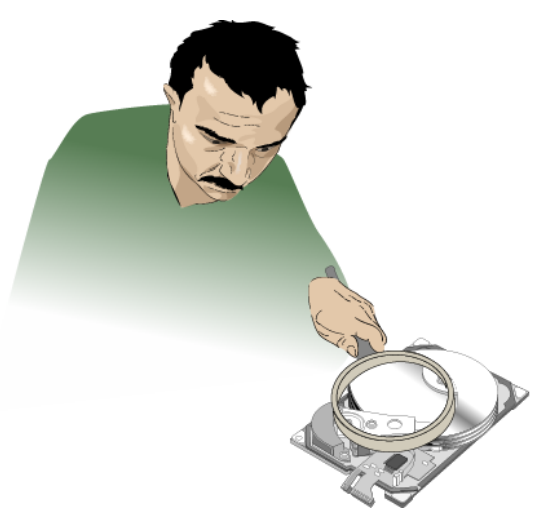

() 2005 Carnegie Mellon University

Module 2: Understanding File Systems and Building a First Responder Toolkit

\subsection{Summary}

From this module you have been exposed to some basic hard drive geometry, and an overview of how the hard drive works with an OS's file system to store data. You should understand that files leave traces when deleted and know that those file artifacts are hidden in swap and slack space. Together these provide for a better understanding of the fragile nature of forensic evidence.

Building a first responder toolkit using this module's methodology provides you with a baseline understanding of the different systems in the work environment. Establishing and using this repeatable, defined collection process is essential if, based on collected evidence, you take administrative action against an individual or submit evidence to a court of law. Without a repeatable, defined process, your evidence will be challenged. Investing the time and effort in creating a first responder toolkit pays off when an incident occurs and the scramble to collect evidence ensues. 


\section{Review}

1. (True/False) Low-level formatting installs Windows, and Linux installs the file system on the hard drive.

2. (True/False) Clusters are sized to specifically fit a file.

3. (True/False) Statically-linked tools are preferred over dynamically-linked tools.

4. Name two locations from where an investigator might recover a file or file information after deletion.

5. (True/False) A basic copy or image of a drive contains all of the file information present.

6. (True/False) With Windows NTFS, wiping a file permanently remove all traces.

\subsection{Review}

1. False. Low-level formatting occurs at the factory and installs the sectors and tracks on the disk. High-level formatting establishes the OS's file system.

2. False. Clusters are a fixed size with incorporate several sectors. Their exact size is determined by the Windows version and size of the hard drive.

3. True. Statically-linked is preferred because all file dependencies are trusted and not accessed from the suspicious computer's hard drive.

4. Swap space (virtual memory), slack space, unallocated space, windows artifacts (i.e., multiple data streams, temp files, registry, shortcuts).

5. False. Only a bit-by-bit or duplicate image provides all binary data from a disk. Using the basic copy command or imaging only reproduces the allocated space.

6. False. Windows artifacts and alternate data streams in the NTFS hold keys to a file's existence even after a DoD standard wipe. 


\section{Module 3: Collecting Volatile Data}

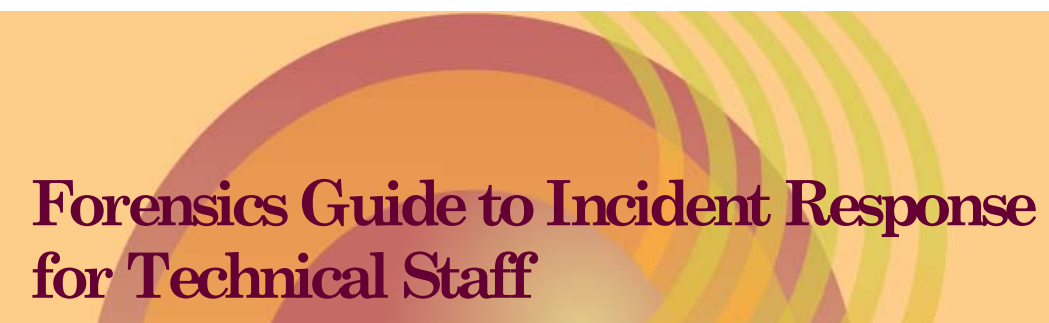

Module 3:

Collecting Volatile Data

CERT ${ }^{\circledR}$ Training and Education

Networked Systems Survivability

Software Engineering Institute

Carnegie Mellon University

Pittsburgh, PA 15213-3890

(c) 2005 Carnegie Mellon University

(®) CERT, CERT Coordination Center, and Carnegie Mellon are registered in the U.S. Patent and Trademark Office

This material is approved for public release. Distribution is limited by the Software Engineering Institute to attendees.

\subsection{Introduction}

This module briefly reviews some best practices, techniques, and tools for collecting volatile data from live Windows and Linux systems. It also explains the importance of collecting volatile data before it is lost or changed on the suspicious computer.

Traditionally, computer forensics has focused on researching, developing, and implementing proper techniques, tools, and methodologies to collect, store, and preserve sensitive data that is left on a system's hard drive(s).

First responders (system and network administrators, law enforcement officers, and others) generally react to a computer security incident by properly shutting down and securing the suspicious computer. After shutdown, persistent data on the computer's hard drive or other persistent storage media devices is collected. But the shutdown precludes the collection of volatile data, critical sources of information that are lost when the computer is rebooted or shut down. To collect volatile data, the first responder needs a running computer system. 
In recent years, law enforcement and information security professionals have invested significant resources into developing forensic data retrieval tools like Guidance Software's EnCase, Access Data's FTK, and NTI's Law Enforcement Suite. However, these tools and techniques focus almost exclusively on extracting and analyzing nonvolatile (persistent) data from hard drives. Little attention has been given to developing a portable, safe, all-in-one application for collecting volatile data from a live machine.

There are many open source tools that allow the first responder to pull volatile information from a live system. However, most open source tools are specifically designed to collect portions of volatile data, not the complete set of volatile data (e.g., Sysinternals' PsList generates details about processes that are currently running).

First responders need to identify and gather a set of open source tools (including native commands) that collect the range of volatile data. Then they can use scripting languages (e.g., Perl, VBScript, batch files, and shell scripts) to execute all tools and commands and automate the data collection. 
Objectives

Role of a first responder

What volatile data is

Order of volatility

Why volatile data is important to collect

First responder mistakes

Types of volatile data

Volatile data collection methodology

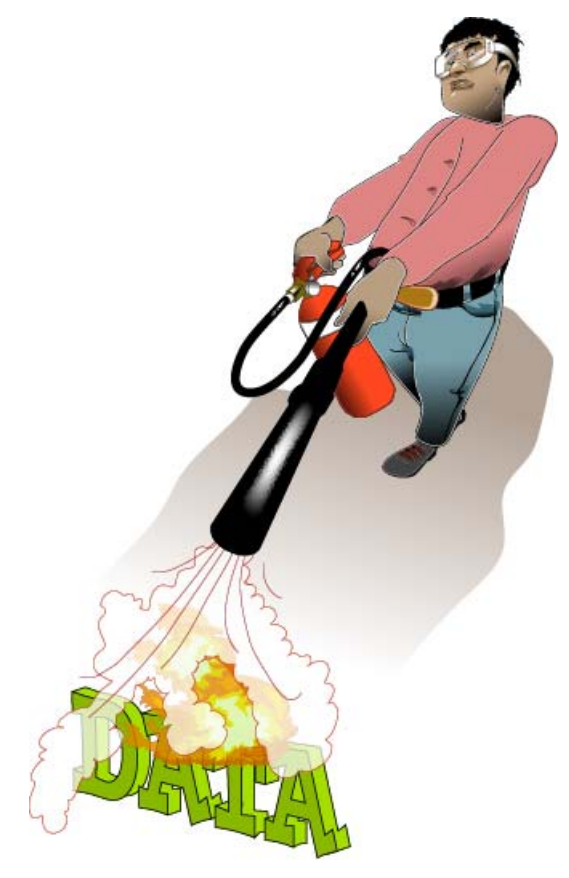

๑) 2005 Carnegie Mellon University

Module 3: Collecting Volatile Data

\subsection{Objectives}

This module focuses on the critical role of the first responder in collecting volatile data from a live suspicious machine during a security incident. Common first responder mistakes are also highlighted so that you do not hamstring your efforts.

Discussions of volatile data characteristics (e.g., what it is, the different types, the order of volatility, and why it should be collected) and the tools used to collect it should give the first responder a working knowledge of the subject.

The bulk of this module introduces the methodology and specific tools for collecting volatile data. It includes steps for safely collecting volatile data so that you can determine the current state of the computer, the root cause of the incident, and what to do next. 


\section{Role of a First Responder}

\section{Essentially the first person notified and reacting to the security incident}

\section{Responsibilities:}

- Determine the severity of the incident

- Collect as much information about the incident as possible

- Document all findings

- Share this collected information to determine the root cause

(c) 2005 Carnegie Mellon University

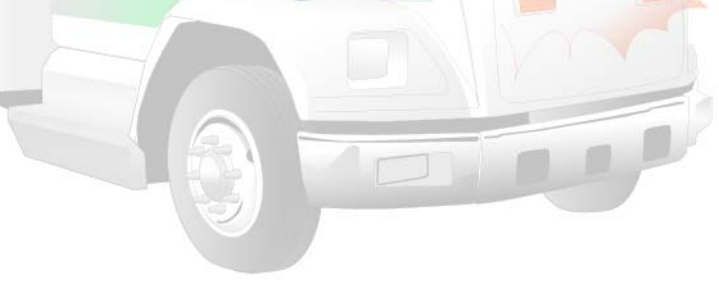

Module 3: Collecting Volatile Data

\subsection{Role of a First Responder}

The first responder to a security incident has a unique and important position to play. He will generally be the first person notified and the first person to react. In most cases, the first responder is a system or network administrator, but by definition it is whoever is assigned to handle security incidents and determine their root causes.

The first responder should be prepared and his actions should be planned. Deliberate - rash or hurried actions could damage potential evidence. He should have a first responder toolkit and a predetermined incident response plan to follow regardless of the type of data (volatile, persistent, or both) being collected. 


\title{
What is Volatile Data?
}

\author{
Definition: Any data stored \\ in system memory that will be \\ lost when the machine loses \\ power or is shut down
}

Location: Registers, cache, and RAM (this module focuses on RAM)
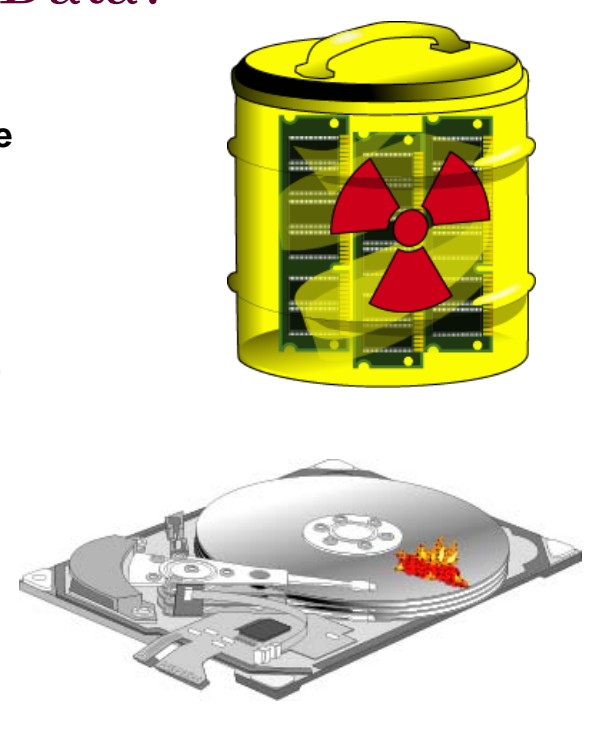

๑) 2005 Carnegie Mellon University

Module 3: Collecting Volatile Data

\subsection{What is Volatile Data?}

Volatile data is stored in system memory (e.g., system registers, cache, RAM) and is lost if the machine loses its power, is shut down, or rebooted. Volatile data collection focuses on collecting data (primarily from RAM) that could be lost if the computer is shut down or rebooted. Volatile data should be collected if you are not sure why a computer is acting abnormally, if you notice suspicious user activity or if you have been alerted that a rule or policy has been violated such as firewall or IDS alert. In any case, the first response to a computer security incident should be to collect volatile data and analyze the results to determine a next course of action.

Persistent data resides in the system's hard drives or other nonvolatile storage devices (e.g., connected USB drives, flash cards, CD-ROMs, and other external hard drives) and is typically not lost when the machine is shut down or rebooted. Generally, persistent data should be collected when it is clear that evidence related to the computer security incident resides in the persistent storage areas.

For both collection strategies, preventing contamination of the suspicious computer is an ongoing issue. For persistent data collection, contamination can be controlled by current techniques, tools, and methodologies. One such tool is dd.exe, a free imaging utility developed by George M. Garner Jr. that has the capability to create a bit-by-bit copy of the suspicious computer's hard drive. If you create MD5 checksums of the hard drive before and after using a utility like DD, you can compare them and authenticate the copy. For volatile data collection, contamination is harder to control because tools and commands may change 
file access dates and times, use shared libraries or DLLs, trigger the execution of malicious software (malware), or-worst case-force a reboot and lose all volatile data. These potential effects should have been uncovered and documented during Module 2's tool testing phase of creating a first responder toolkit.

Obviously, you can not make a bit-by-bit copy of a live computer. But if you use best practices and a first responder toolkit, you can use the collected data and a reproducible methodology to reconstruct a logical representation of the current state of the suspicious system. 


\section{Order of Volatility}

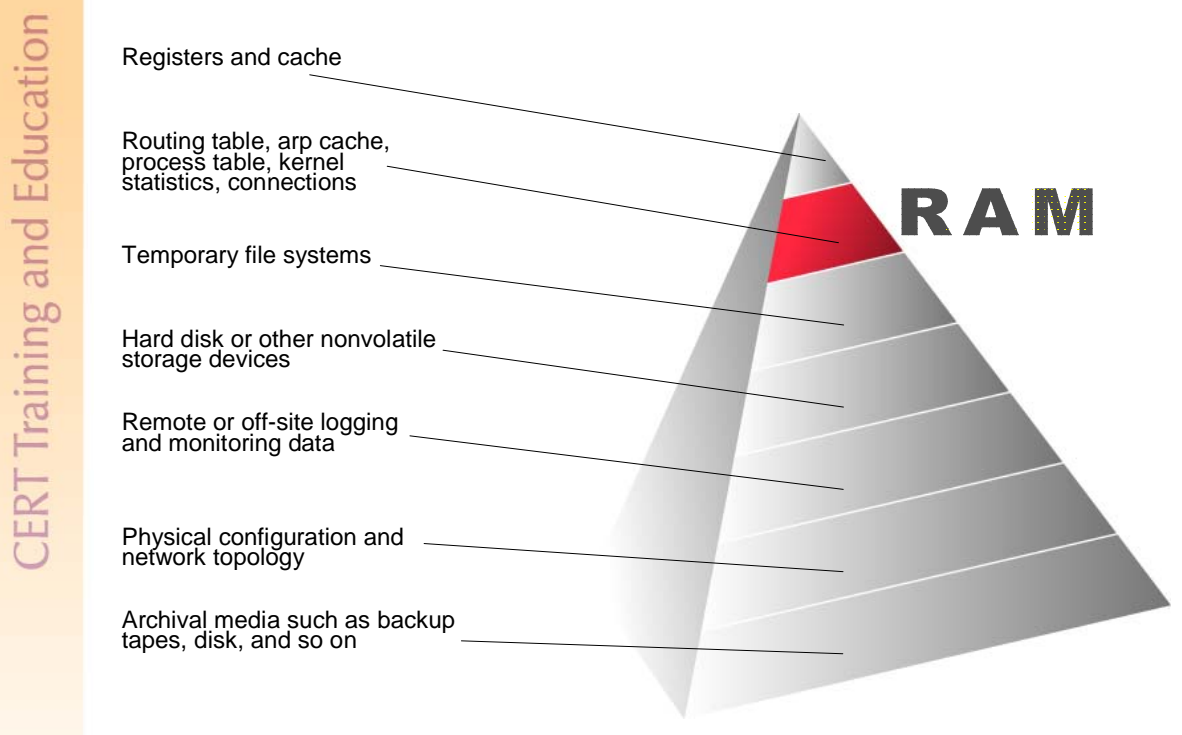

(c) 2005 Carnegie Mellon University

Module 3: Collecting Volatile Data

\subsection{Order of Volatility}

As you collect data (i.e., potential evidence) from a live computer, consider the data's order of volatility: that is, you collect data that has the highest chance of being changed, modified, or lost first. The order of volatility for Windows and Linux computers is the same [Brezinski 02].

This module focuses on data stored in RAM. It shows how to collect this data safely and securely while minimizing the footprint you leave on the suspicious computer. 


\section{Why is Volatile Data Important?}

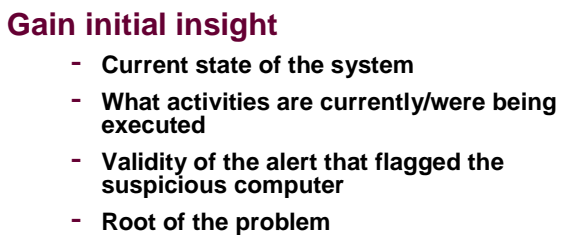

- Current state of the system

- What activities are currently/were being executed

- Validity of the alert that flagged the suspicious computer

- Root of the problem

Determine a logical timeline of the incident

- Identify the time, date, and user responsible for the security incident

\section{Determine next step}

- Decide whether a full collection of the persistent data on the suspicious computer is necessary

One chance to collect - After the system is rebooted or shut down,
it's too late!

(c) 2005 Carnegie Mellon University

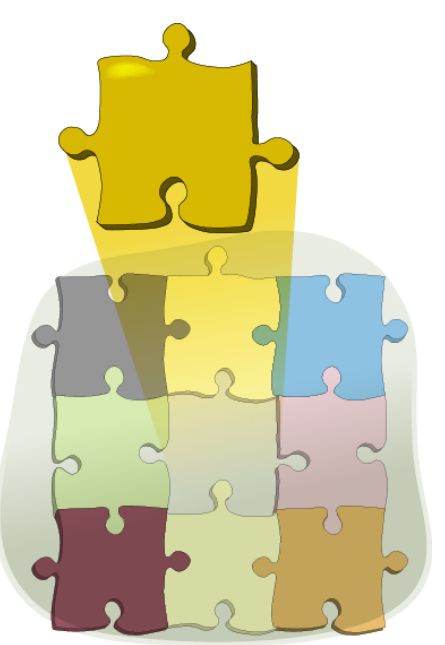

Module 3: Collecting Volatile Data

\subsection{Why is Volatile Data Important?}

Volatile data stored in RAM must be obtained immediately after the security incident is reported so that data about the current state of the computer-including logged on users, running processes, and open connections - can be collected. This data helps you determine a logical timeline of the security incident and the possible users responsible.

After you collect as much volatile data as you can, decide on the next step. For example, you may have enough data to determine that the initial alert that flagged this computer is valid. You may then discover a rogue process, a likely root cause. And if the rogue process is running on a critical asset, you will have to remove it remotely and then verify if it is malicious.

The key is to view the suspicious computer as completely unreliable until—using the methodology and tools described in this module-some level of trust is established. 


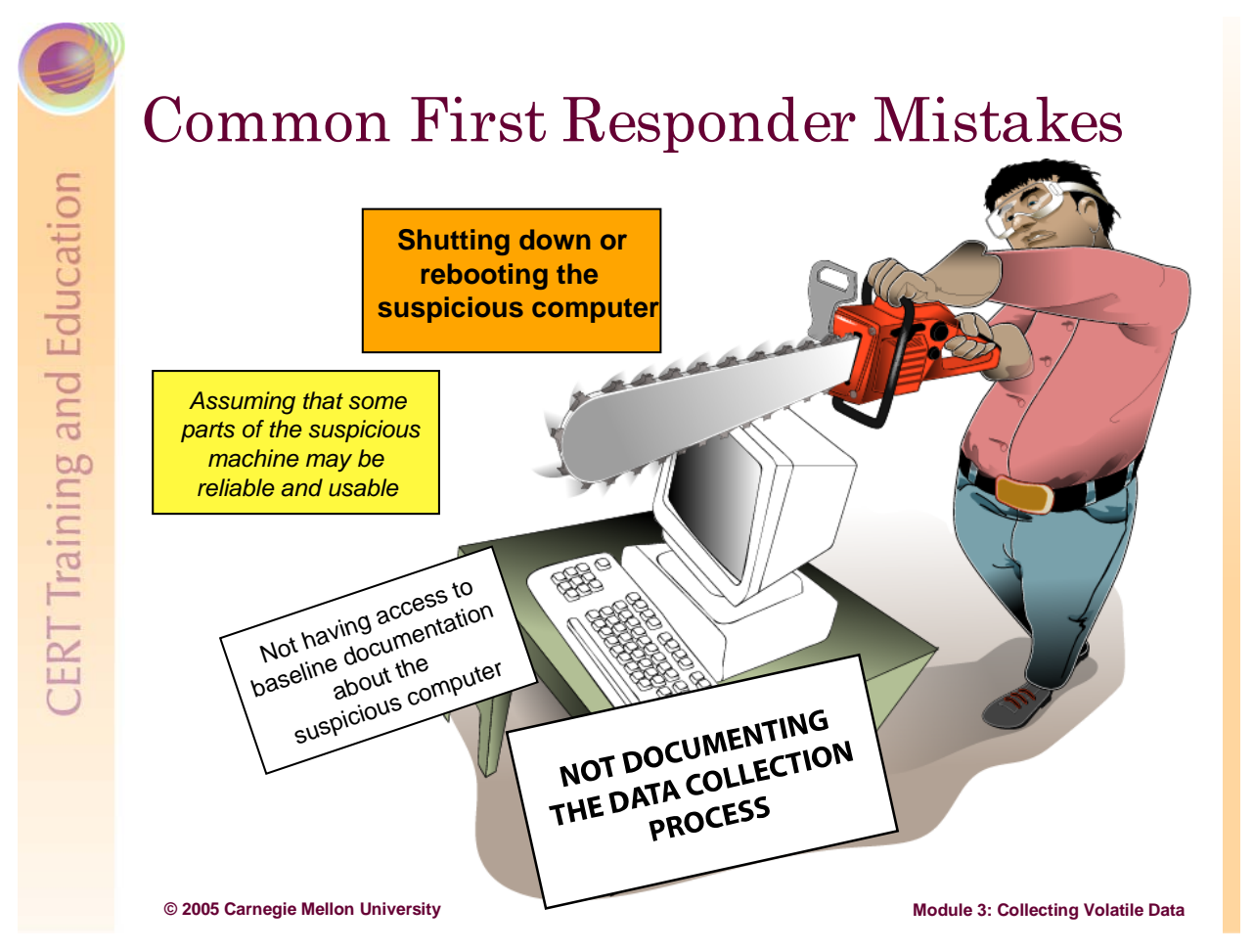

\subsection{Common First Responder Mistakes}

Because many first responders play dual roles as system and network administrators, they have little time to properly plan for and then handle security incidents. Swamped with routine day-to-day tasks, they may never consider developing a volatile data collection methodology. For the methodology to be successful, though, first responders should avoid the two most critical mistakes:

- $\quad$ shutting off or rebooting the machine

In this case, all volatile data is lost: connections and running processes are closed and MAC times are changed. Closing an open shell or terminal may have the same effect.

- assuming that some parts of the suspicious computer are reliable

In this case, using native commands on the suspicious computer may trigger Trojans, time bombs, and other malware to delete key volatile data. 


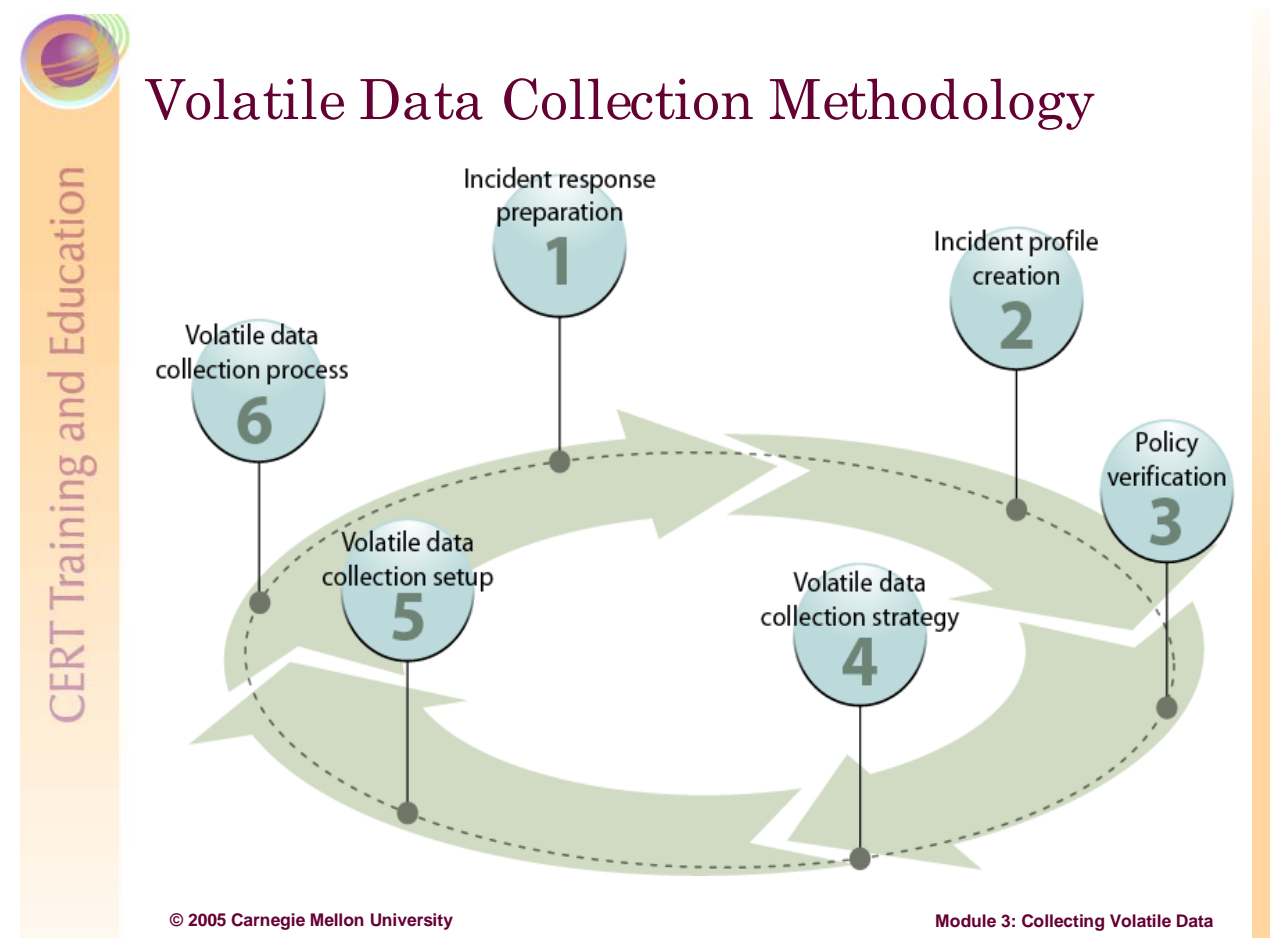

\subsection{Volatile Data Collection Methodology}

Before we introduce specific tools, we need to provide a sound volatile data collection methodology. Develop and use this methodology so that you collect volatile data the first time: you only get one chance.

Having a methodology is important. It provides a documented approach for performing activities in a coherent, consistent, accountable, and repeatable manner [ICH 03].

Harlan Carvey [Carvey 04] states that most system administrators do not have a clear process to follow when responding to security incidents. Often, an administrator's default action, due to lack of knowledge or time, is to remove the OS and reload it from the original installation media. Several drawbacks are that a root cause analysis is never done and reloading the OS takes the system out of service. To avoid these drawbacks, an initial forensic collection of the volatile data on the live system should be performed.

What follows is a six part methodology for first responders to use when volatile data has to be collected from a live computer. The actual data collection is not performed until the final step. 


\section{Step 1: Incident Response Preparation}

\section{Forensic Tool Test Bed}

First responder toolkit

Creation of Collection policies

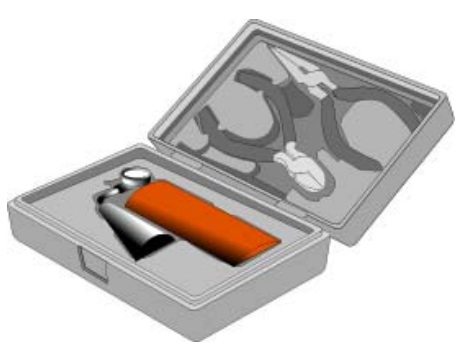

() 2005 Carnegie Mellon University

Module 3: Collecting Volatile Data

\subsubsection{Step 1: Incident Response Preparation}

As a first responder, you can never eliminate or even anticipate every type of security incident or threat. You can, however, be prepared to react successfully and swiftly so that you can collect all types of volatile data from a live computer. Before an incident occurs, the following items should be in place.

- a first responder toolkit (response disk)

- $\quad$ an incident response team (IRT) or designated first responder

(NIST's guidelines [NIST 01] are a good resource)

- forensic-related policies that allow for forensic collection 


\section{Step 2: Incident Documentation}

\section{Incident profile}

\section{Forensic collection logbook}

First responder toolkit documentation

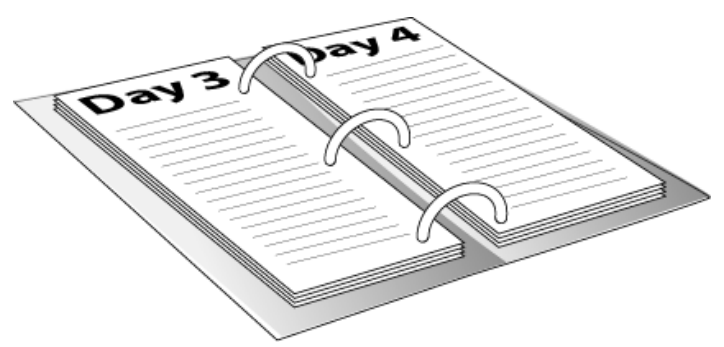

๑) 2005 Carnegie Mellon University

Module 3: Collecting Volatile Data

\subsubsection{Step 2: Incident Documentation}

In your incident documentation, be straightforward and consistent so that your generated profiles and logs are organized and readable. For example, use consistent naming conventions for forensic tool output. Stamp your logs with the time, date, and identity of the person performing the forensic collection. These best practices provide an audit trail of the chain of custody for the security incident.

\subsubsection{Incident Profile}

Document as much information about the security incident as possible. The checklist shown in the graphic is taken from Foundstone's Incident Notification Checklist [Mandia 01]. Use the following profile information to determine your volatile collection strategy or next course of action.

- How was the incident detected?

- What is the scenario for the incident?

- What time did the incident occur?

- Who or what reported the incident?

- What hardware and software are involved?

- Who are the contacts for involved personnel?

- How critical is the suspicious computer? 


\subsubsection{Forensic Collection Logbook}

During your forensic collection, use a logbook (i.e., a text file or notebook) to document all of your actions. The logbook provides a timeline of events and an audit trail if unforeseen things occur during the collection. Include the following:

- who is performing the forensic collection

- history of executed forensic tools and commands

- generated forensic tool and command output

- date and time of the executed commands and tools

- expected system changes or effects (i.e., changed MAC times for specific files) as first responder tools are executed (these effects were discovered in Module 2)

\subsubsection{First Responder Toolkit Logbook}

As you collect volatile data, refer to the first responder toolkit logbook that was created in Module 2. It contains information about forensic tool usage, output, and system affects for each collection tool or native command you tested. Use the logbook to determine which tools are the best for your situation. 


\section{Step 3: Policy Verification}

\section{Determine your authority to collect}

Determine your manner to collect

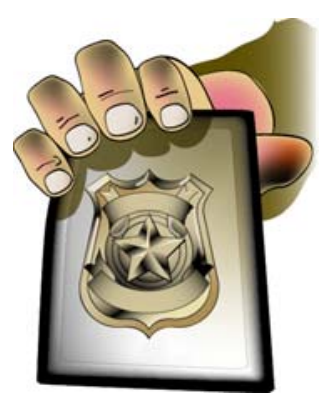

๑) 2005 Carnegie Mellon University

Module 3: Collecting Volatile Data

\subsubsection{Step 3: Policy Verification}

Make sure that any actions you plan to take as first responder do not violate any existing computer and network usage policies. In particular, make sure that you do not violate any rights of the registered owner or user of the suspicious computer.

- Examine any policies the user of the suspicious computer has signed.

- Determine if the user consented to monitoring either through a signed policy statement or through system banners.

- As necessary, determine the legal rights (including a review of federal statutes) that the user has so that you can determine what forensic capabilities and limitations you have. (See Module 1.)

We recommend that first responders read the following CERT Coordination Center publications before collecting any data:

- Establish Policies and Procedures for Responding to Intrusions http://www.cert.org/security-improvement/practices/p044.html

- $\quad$ CERT $^{\circledR}$ Advisory CA-1992-19 Keystroke Logging Banner http://www.cert.org/advisories/CA-1992-19.html

- Steps for Recovering from a UNIX or NT System Compromise http://www.cert.org/tech_tips/win-UNIX-system_compromise.html\#A.1 


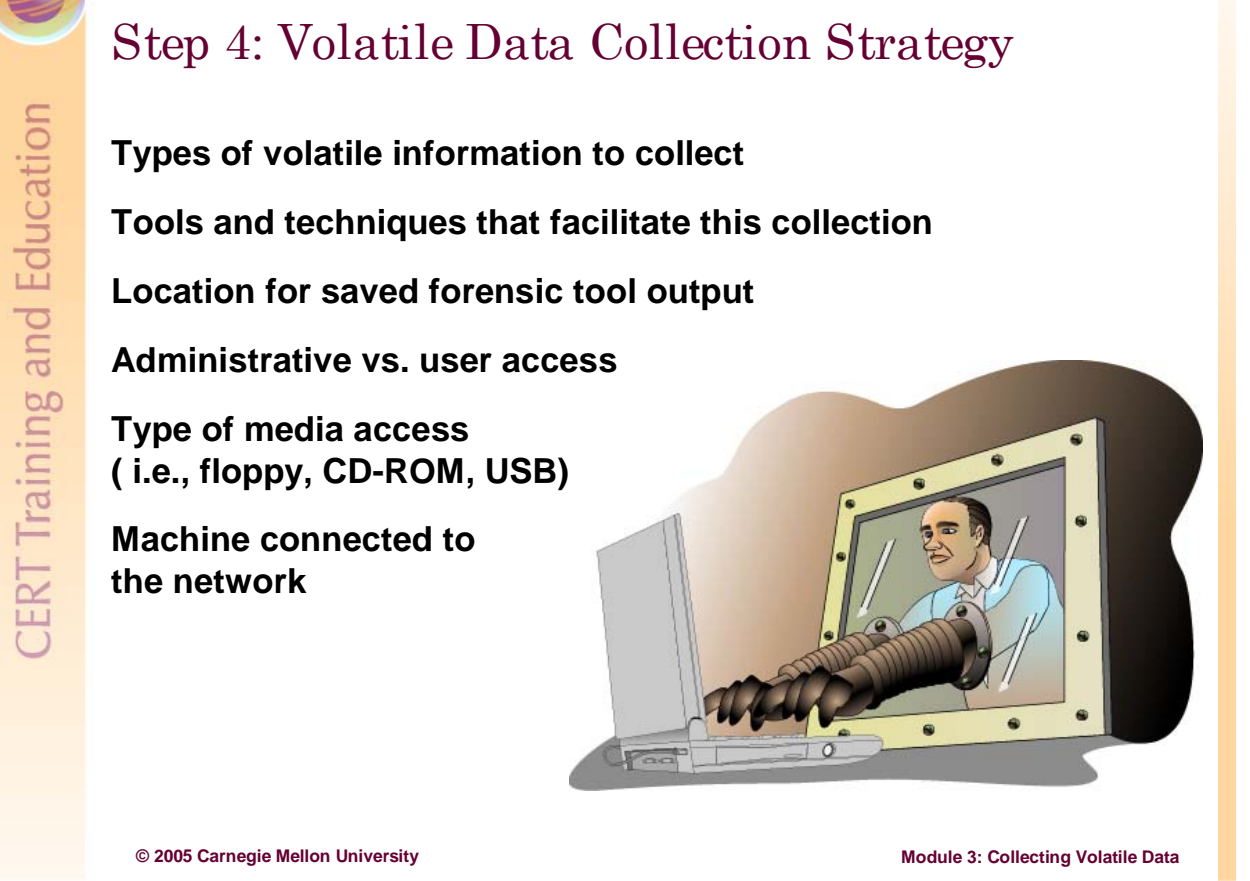

\subsubsection{Step 4: Volatile Data Collection Strategy}

No two security incidents will be the same. Use the first responder toolkit logbook and the questions from the graphic to develop a collection strategy that will address your situation and leave the smallest possible footprint on the suspicious computer. 


\section{Step 5: Volatile Data Collection Setup}

Establish a trusted command shell

Establish the transmission and storage method

Ensure the integrity of forensic tool output

๑) 2005 Carnegie Mellon University

Module 3: Collecting Volatile Data

\subsubsection{Step 5: Volatile Data Collection Setup}

\subsubsection{Establish a Trusted Command Shell}

As stated earlier, you do not want to make that common first responder mistake of trusting any parts of the suspicious computer. Therefore, do not open or use a terminal or command shell from the suspicious computer. This will minimize the footprint on the suspicious computer and prevent the triggering of any kind of malware that have been installed on the system.

The response disk that was developed during Module 2 should include a trusted command shell along with the forensic tools.

If the suspicious computer is a Linux system running $\mathrm{X}$-Windows, exit $\mathrm{X}$-Windows. Some UNIX flavors have a vulnerability that allows attackers to log your keystrokes [Mandia 01].

\subsubsection{Establish a Method for Transmitting and Storing the Collected Information}

Because you may not have enough space on your response disk (i.e., USB drive, floppy, etc.) to collect forensic tool output, identify and document how that data will be transmitted from the live suspicious computer to the remote data collection system. Two reliable freeware utilities that transmit data remotely via a network are Netcat and Cryptcat.

Netcat is a networking utility that reads and writes data across network connections using the TCP/IP protocol. It is designed as a reliable "back-end" tool that can be used directly or 
driven by other programs and scripts. It is also a feature-rich network debugging and exploration tool, able to create almost any kind of connection you need and with several interesting built-in capabilities [GNU 04].

Cryptcat is a lightweight version of netcat with integrated transport encryption capabilities. It ensures data confidentiality while transmitting it over networks and is particularly useful if you suspect a network sniffer.

\subsubsection{Ensure the Integrity and Admissibility of the Forensic Tool Output}

To ensure the integrity and admissibility of forensic tool output, compute an MD5 hash of it. When an electronic file is transferred or sent to another party, a rehash verifies its integrity.

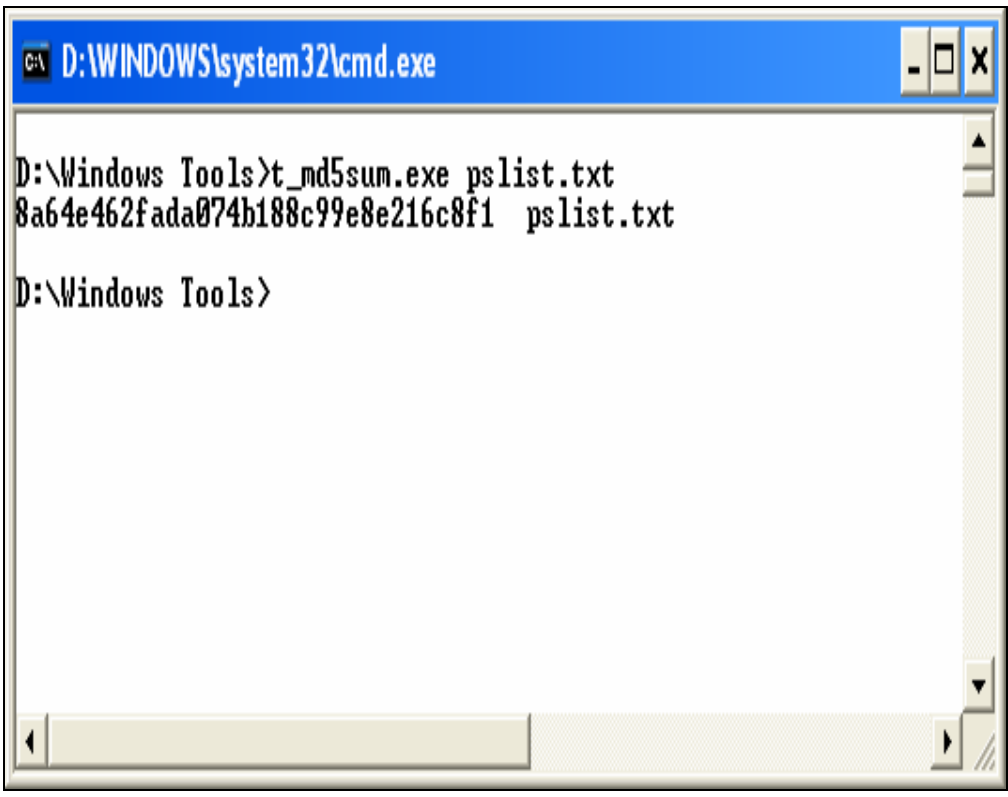

Figure 8: An MD5 Hash 


\section{Step 6: Volatile Data Collection Process}

1. Collect uptime, date, time, and command history for the security incident.

2. As you execute each forensic tool or command, generate the date and time to establish an audit trail.

3. Begin a command history that will document all forensic collection activities.

4. Collect all types of volatile system and network information.

5. End the forensic collection with date, time, and command history.

๑) 2005 Carnegie Mellon University

Module 3: Collecting Volatile Data

\subsubsection{Step 6: Volatile Data Collection Process}

Now you are ready to collect volatile data. The remaining sections of this module describe each data type and specific tools for collecting it. 


\section{Types of Volatile Information}

Volatile System Information: A collection of information about the current configuration and running state of the suspicious computer

Volatile Network Information: A collection of information about the network state of the suspicious computer

\subsection{Types of Volatile Information}

Two types of volatile data that can be collected from the suspicious computer: system information and network information. The forensic collection tools that are described in the balance of this module are organized by these two data types. 


\section{Volatile System Information}

System profile

Current system date and time

Command history

Current system uptime

Running processes

Open files, start up files, clipboard data

Logged on users

DLLs or shared libraries

๑) 2005 Carnegie Mellon University

Module 3: Collecting Volatile Data

\subsubsection{Volatile System Information}

This list of volatile system information types, while not exhaustive, reveals enough details about the suspicious computer's current state, configuration, and users for you to begin to understand the severity, probable causes, and perpetrator of the security incident. 


\title{
System Profile
}

\author{
Definition: Describes \\ the suspicious \\ computer's \\ configuration
}

Purpose: To document

the baseline

configuration for

comparison purposes

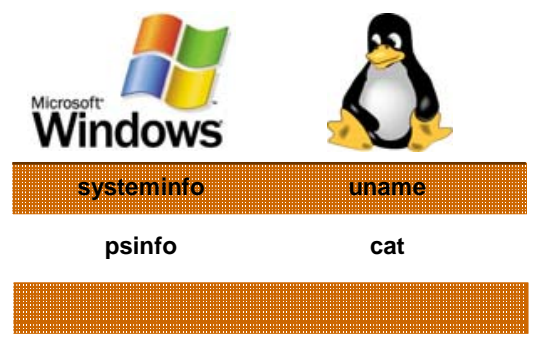

๑) 2005 Carnegie Mellon University

Module 3: Collecting Volatile Data

\subsubsection{System Profile}

The system profile describes the baseline configuration of the suspicious computer. Ideally, system and network administrators create these profiles for all computers within their IT and network infrastructures, but if one doesn't already exist for the suspicious computer, create it now. This profile provides a physical "snapshot" of the computer and is often requested by a forensic examiner.

The system profile includes the following details about the configuration of the suspicious computer:

- OS type and version

- system installation date

- registered owner

- $\quad$ system directory

- total amount of physical memory

- pagefile location

- installed physical hardware and configurations

- installed software applications

The following sections describe tools and commands for collecting this information. 


\section{Systeminfo.exe (Windows)}

The native systeminfo.exe command allows you to create a system profile that includes

- registered owner

- original install date

- $\quad$ system uptime

- BIOS version

- $\quad$ system directory

- logon server

- number of network cards installed

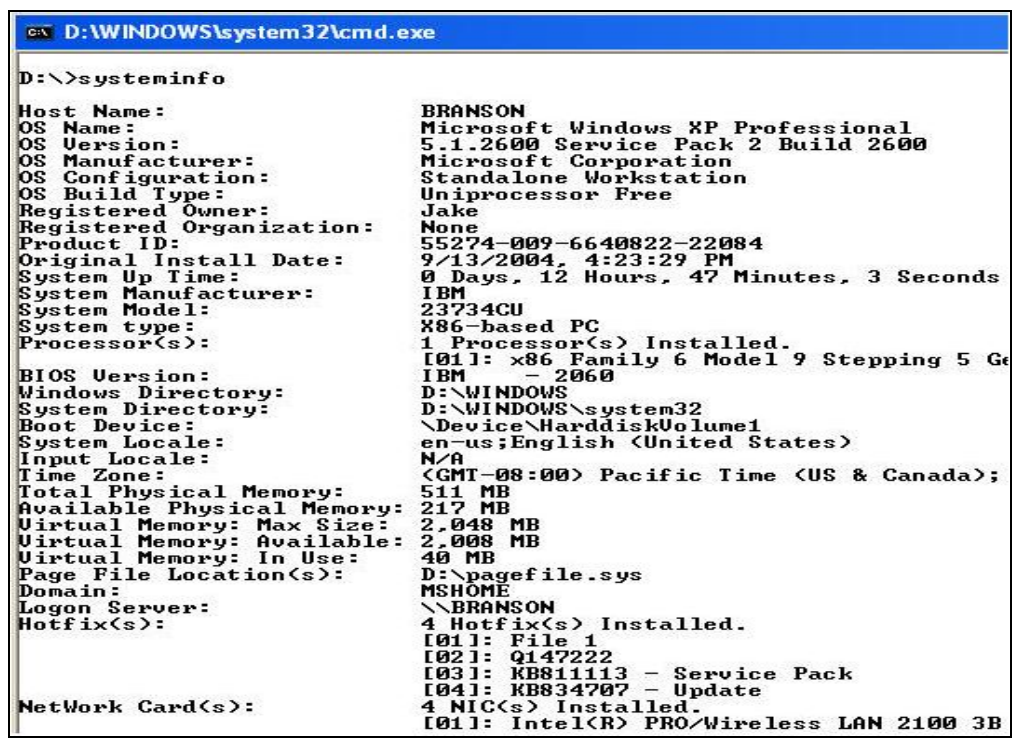

Figure 9: The systeminfo Command

\section{PsInfo (Windows)}

Sysinternals' PsInfo also allows you to create a system profile to include installed software packages and hot fixes, which the Systeminfo.exe command does not show you. By default, the PSInfo creates profiles of the local system. You can run it remotely by specifying a username and password of the suspicious computer.

PsInfo relies on remote registry access to obtain data on non-local systems. The remote system must be running the Remote Registry service and the account from which you run PsInfo must have access to the HKLM $\backslash$ System portion of the remote registry [Russinovich 04a].

PsInfo is versatile. Use it to query for types of installed software, hot fixes, hard drive size, and remaining unused space. In Figure 10, we used Psinfo to query the suspicious computer for the number of applications installed. 


\begin{tabular}{|c|c|}
\hline cil D:IWINDOWSIsystem32lemd.exe & $-\nabla x$ \\
\hline$D: \backslash W T 00 l s>P s i n f 0 . e x e-s$ "sof tware" & 4 \\
\hline $\begin{array}{l}\text { PsInf o v1.62 - Local and remote systen information viewer } \\
\text { Copyright (C) } 2001-2094 \text { Mark Russinovich } \\
\text { Sysinternals - ww.sysinternals.com }\end{array}$ & \\
\hline System information for IIBRANSON: & \\
\hline $\begin{array}{l}\text { Applications: } \\
\text { ATI - Software Uninstall Utility } 6.14 .10 .1007 \\
\text { ATI Control Panel 6.14.10.5093 } \\
\text { ATI Display Driver } 7.983 .2-040513 a-015558 \mathrm{C}-\mathrm{IBM} \\
\text { Access IBM 4.0 } \\
\text { Access IBM Tools } 4.0 \\
\text { Adobe Reader } 6.0 .1 \text { 006.000.001 } \\
\text { Agere Systems AC'97 Modem 2.1.31 }\end{array}$ & \\
\hline
\end{tabular}

Figure 10: The PsInfo Command

\section{Cat (Linux)}

The native cat command allows you to create a system profile by displaying file contents of the version, uptime, meminfo, filesystems, and cpuinfo files located in the proc file.

\begin{tabular}{|c|c|}
\hline \multicolumn{2}{|l|}{ V rootelocalliost.jproc } \\
\hline \multicolumn{2}{|l|}{ Elle Edit View Ierminal Go Help } \\
\hline \multirow{4}{*}{\multicolumn{2}{|c|}{$\begin{array}{l}\text { [rootelocalhost proc]\# cat version uptine neninfo filesystems c } \\
\text { Linux version 2.4.18-14 (bhcompile@stripples. devel.redhat.con) } \\
03 \text { (Red Hat Linux 8.0 3.2-7)) \#1 Wed Sep 4 13:35:50 EDT } 2002 \\
3586.0046 .3396\end{array}$}} \\
\hline & \\
\hline & \\
\hline & \\
\hline \multicolumn{2}{|c|}{ total: used: free: shared: buffers: cached: } \\
\hline \multicolumn{2}{|l|}{ Men: 307015680139620352167395328} \\
\hline Swap: 534634496 & \\
\hline MenTotal: & \\
\hline $163472 \mathrm{kB}$ & \\
\hline Menshared: & \\
\hline Buffers: & \\
\hline
\end{tabular}

Figure 11: The cat Command

\section{Uname (Linux)}

The native uname command allows you to create a system profile that includes machine name, machine's network node hostname, the type of processor, the OS release, and the OS kernel version. 


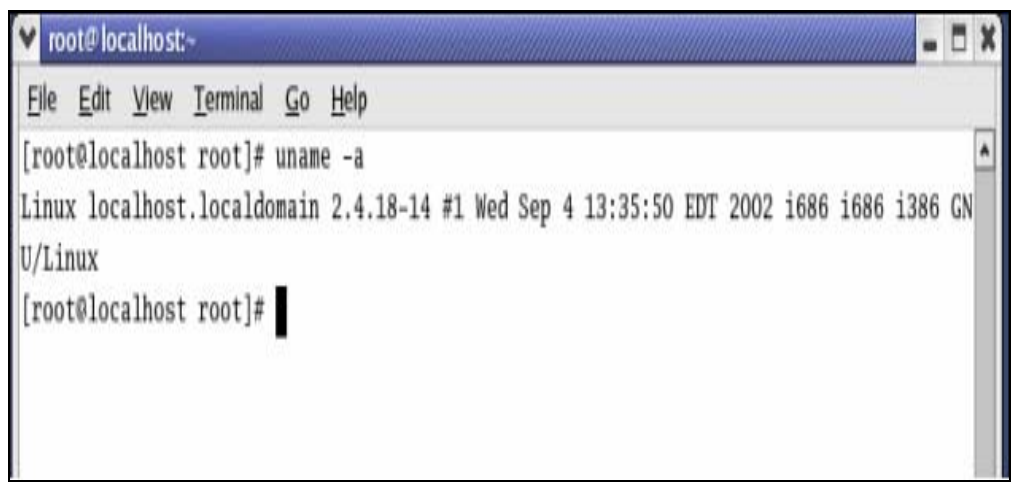

Figure 12: The uname Command

If you did not know the kernel version on the suspicious computer, this information is particularly important. An old kernel that hasn't been patched or recompiled may have vulnerabilities that are well documented by the vendor. 


\section{Current System Date and Time and Command History}

\section{Purpose:}

\section{Documents the start of your data collection \\ Gain insight to recent command or terminal activity}

\section{Documents the end of your}

data collection

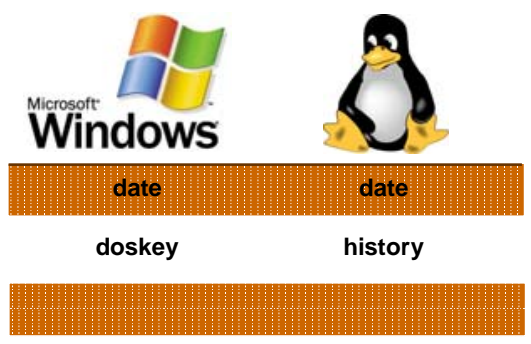

(c) 2005 Carnegie Mellon University

Module 3: Collecting Volatile Data

\subsubsection{Current System Date and Time and Command History}

The system date, time, and command history are important throughout the forensic collection process. Initially, the first responder uses the current date and time to document when he discovered the suspicious computer and started collecting volatile data. During data collection, he uses the current date and time to document when each forensic tool or command was executed. These date and timestamps are critical to admissible data and easy to obtain from native OS commands and free open source tools.

Figure 13 shows how the native date and time commands can be used with a native forensic command like netstat to document at what time and date you executed the netstat command.

\begin{tabular}{|l|l|}
\hline C:I D:WWINDOWSlsystem32lcmd.exe & $-\square \times$ \\
\hline D: \WTools>netstat $-\mathrm{a} \&$ date $/ \mathrm{t} \&$ \& time $/ \mathrm{t}$ & - \\
\hline
\end{tabular}

\section{$\checkmark$ root@localhost:}

File Édit View Terminal Go $\underline{\text { Help }}$

[root@localhost root]\# netstat -a ; date

Figure 13: date and time Commands Used with netstat

In both Linux and Windows the command history reveals recent activities of the suspicious computer's user(s). It includes a list of recently executed commands performed by a local or remote user within an established terminal or command shell. If the suspicious computer has 
more than one user account, collect the command history for each one. But be advised, Windows history log is virtual while Linux's is persistent.

A collected command history also functions as an audit trail of first responder actions. After you are done collecting data from the suspicious computer, use a native OS command or freeware utility to collect the command history.

As stated earlier, you lose the command history if the open Windows command shell is closed. The same is not true for Linux systems: you can still recover a command history if a terminal is closed.

As you collect a suspicious system's current date, time, and command history do the following:

- Determine if there is any discrepancy between the collected time and date and the actual time and date within your time zone.

- For the current command history, determine if administrative commands have been abused to

- add user accounts

- change NIC configuration

- install unauthorized software applications

- disable security logging, firewalls, or anti-virus software 


\title{
Current System Uptime
}

\author{
Definition: Indicates how \\ long the system has been \\ running since the last \\ reboot.
}

\section{Purpose:}

Helps determine if volatile data collection is worth performing

Indicates whether the security incident occurred during the uptime period

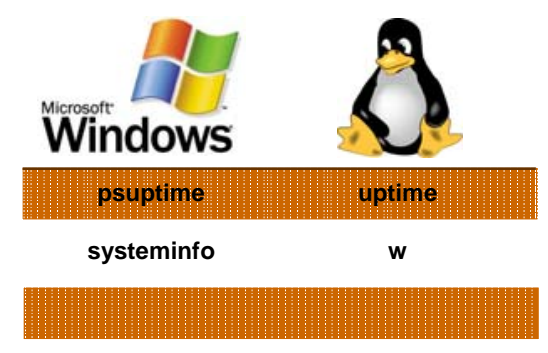

๑) 2005 Carnegie Mellon University

Module 3: Collecting Volatile Data

\subsubsection{Current System Uptime}

System uptime is the period of time that the system has been running since its last reboot. It can help the first responder determine if a volatile data collection is worth performing.

For example, if uptime is only a few minutes, the system has been rebooted recently and it may not be worthwhile to collect volatile data. And it may not be worthwhile if the security incident occurred before the beginning of the uptime period.

\section{PsUptime (Windows)}

SysInternals' PsUptime tells you how long a Windows system has been up. It uses the Performance Data Helper library to easily read the System Up Time performance counter on the local or remote computer.

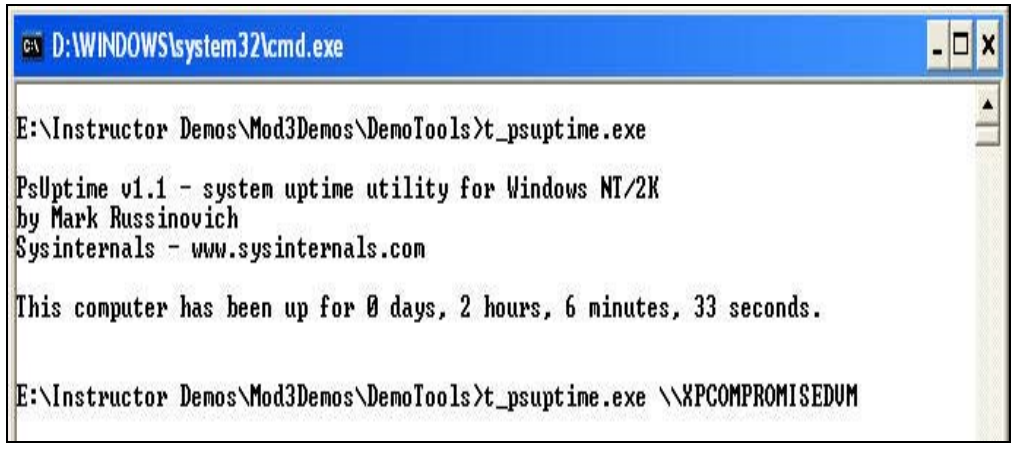

Figure 14: The PsUptime Command 
To gather information on a remote computer, simply specify the computer's name with the command: psuptime.exe $\|<$ computer name>).

\section{Net Statistics (Windows)}

The native net statistics command also allows you to collect uptime information (i.e., net statistics workstation or server).

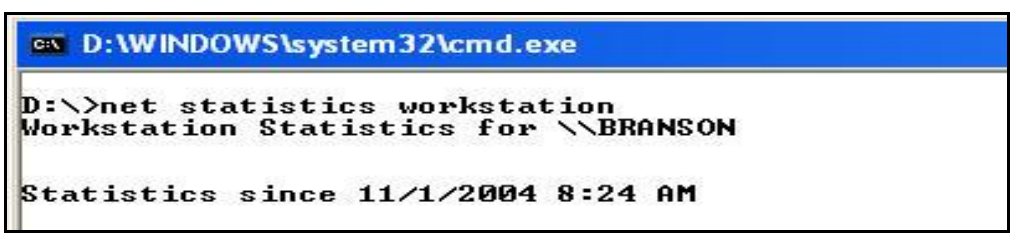

Figure 15: The net statistics Command

\section{Uptime and $W$ (Linux)}

The native uptime and $w$ commands allow you to collect uptime information.

In Figure 16, the machine has been up for approximately one hour and eight minutes from an initial logon at 7:33 p.m.

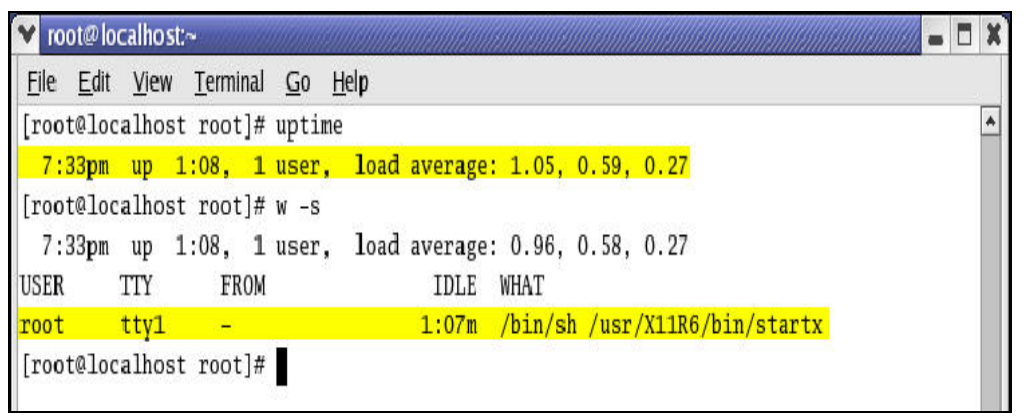

Figure 16: The uptime and w Commands 


\section{Running Processes -1}

\section{Purpose:}

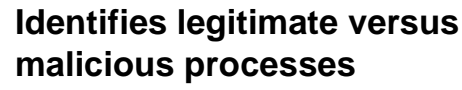

๑) 2005 Carnegie Mellon University

\subsubsection{Running Processes}

Examine the list of currently running processes to identify unnecessary or possible rogue (malicious) processes.

Rogue processes can be created and started independently and are often named for legitimate processes, which can make them extremely difficult to identify. To address this problem, first responders should compare each running process' PID (process identifier) or process filename to a list of legitimate system and application processes.

Uniblue [Uniblue 04] provides a list of legitimate processes for Windows systems on their Web site. 


\section{Running Processes -2}

What to look for:

- Unusual filenames or extra processes (i.e., those not due to normal, authorized activities)

- Processes that have terminated prematurely

- Processes that are run at unexpected times

- Processes that have unusual user identification associated with them

Harlan Carvey [Carvey 04] recommends documenting this set of characteristics for each running process:

- the process' executable image

- the command line used to initiate the process

- how long the process has been running

- the security context that it runs in

- modules or libraries (DLLs) it accesses

- memory that the process consumes

Currently, no single utility comprehensively assesses running processes. Therefore, the following examples show you how to use a combination of commands and utilities to assess process PID (2928). 
1. Use netstat $-a b$ output to determine that the executable file for process PID (2928) is firefox.exe.

\begin{tabular}{|c|c|c|c|}
\hline \multicolumn{4}{|c|}{ C:I D:IWINDOWSIsystem32lcmd.exe } \\
\hline \multicolumn{4}{|l|}{$D: \backslash W T o o l s>n e t s t a t-a b$} \\
\hline \multicolumn{4}{|l|}{ Active Connections } \\
\hline Proto Local Address & Foreign Address & State & PID \\
\hline $\begin{array}{l}\text { TCP branson: } 1572 \\
\text { [f iref ox.exe ] }\end{array}$ & localhost: 1573 & ESTABLISHED & 2928 \\
\hline $\begin{array}{l}\text { TCP branson: } 1573 \\
\text { [f iref ox.exe ] }\end{array}$ & localhost:1572 & ESTABLISHED & 2928 \\
\hline $\begin{array}{l}\text { TCP branson:1818 } \\
\text { [umware.exe] }\end{array}$ & spokane.umware.com:http & CLOSE_WAIT & 2108 \\
\hline
\end{tabular}

Figure 17: Using netstat -ab to Determine Process Executable Image

2. Use Sysinternals' ListDLLs to determine the command line used to execute the process.

\begin{tabular}{|c|}
\hline G:I D:IWINDOWSLsystem32lcmd.exe \\
\hline $\mathrm{D}: \backslash W T 001 s\rangle$ listdlls.exe firefox \\
\hline $\begin{array}{l}\text { ListDLLs U2.23 - DLL lister for Win9x/NT } \\
\text { Copyright (C) 1997-2000 Mark Russinovich } \\
\text { http://ww.sys internals .com }\end{array}$ \\
\hline $\begin{array}{l}\text { firefox.exe pid: } 2928 \\
\text { Conmand line: "D: } \backslash \text { Progran Files } \backslash \text { Mozilla Firefox } \backslash \text { firefox.exe" }\end{array}$ \\
\hline
\end{tabular}

Figure 18: Using ListDLLs to Determine Command Line

3. Use Sysinternals' PsList to discover how long firefox.exe (the 2928 process) has been running.

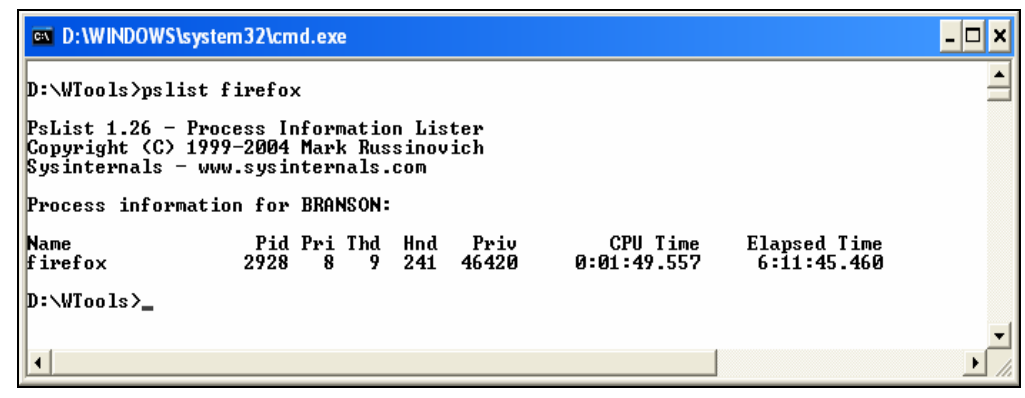

Figure 19: Using PsList to Determine How Long a Process Has Been Running 
4. Use Sysinternals' PsList to discover how much virtual memory the firefox.exe process is currently consuming.

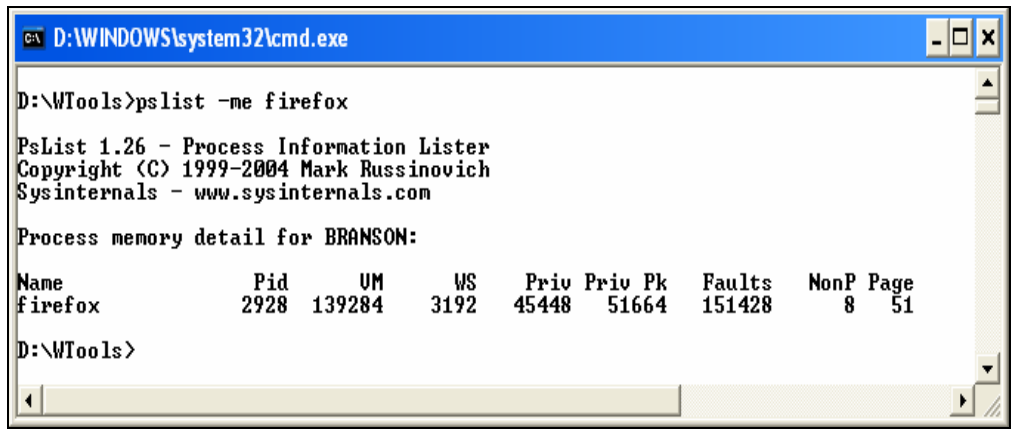

Figure 20: Using PsList to Determine How Much Virtual Memory a Process Is Using

5. Use ListDLLs to discover the currently loaded DLLs for the firefox.exe process.

\begin{tabular}{|c|c|c|c|}
\hline \multicolumn{4}{|c|}{$\begin{array}{l}\text { D: WTools }>\text { listdlls.exe firef ox } \\
\text { ListDLLs U2.23-pLL lister for Win9x/NT } \\
\text { Copyright }\langle\mathrm{C}\rangle \text { 1997-20日9 Mark Russ inovich } \\
\text { http://www.sysinternals.com }\end{array}$} \\
\hline \multicolumn{4}{|c|}{$\begin{array}{l}\text { firefox.exe pid: } 2928 \\
\text { Command line: "D: } \\
\end{array}$} \\
\hline 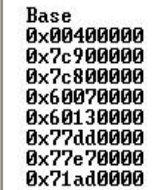 & $\begin{array}{l}\text { Size } \\
0 \times 661000 \\
0 \times b 9000 \\
0 \times 4000 \\
0 \times 54000 \\
0 \times 26000 \\
0 \times 9 b 000 \\
0 \times 91000 \\
0 \times 9000\end{array}$ & $\begin{array}{l}\text { Uersion } \\
0.10 .0009 .0090 \\
5.01 .2609 .2180 \\
5.01 .2600 .2180 \\
4.00 .0009 .0090 \\
4.05 .0009 .0090 \\
5.01 .2600 .2180 \\
5.01 .2609 .2180 \\
5.01 .2600 .2180\end{array}$ & 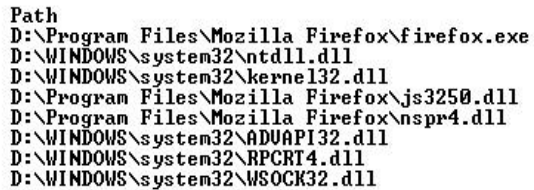 \\
\hline
\end{tabular}

Figure 21: Using ListDLLs to Discover the Currently Loaded DLLs for a Process

\section{Pulist (Windows)}

Pulist output can be examined for unexpected process filenames and unusual user identifications. In Figure 22, the following four processes are well-known rogues: tini.exe, klogger.exe, svchost1.exe, and qfskrtwj.exe

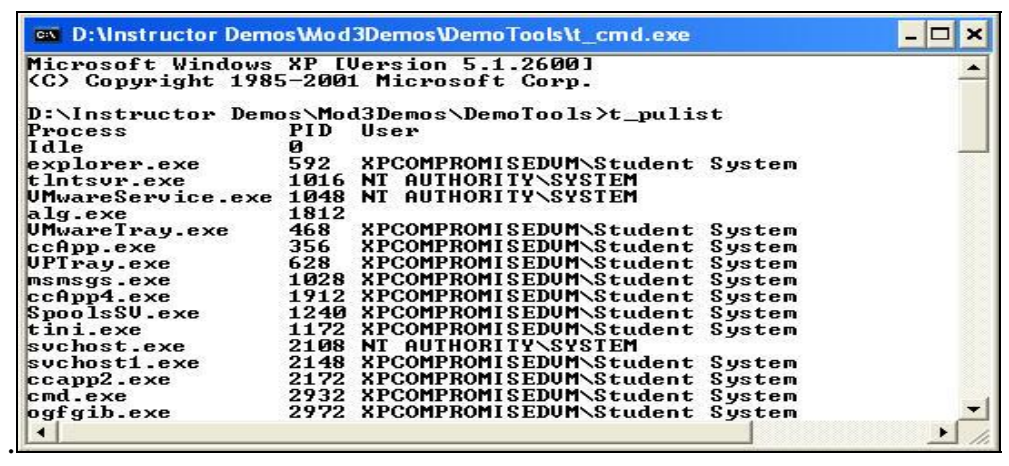

Figure 22: Pulist Output 


\section{Tlist (Windows)}

Windows NT Server Resource Kit includes tlist.exe, which lists active tasks and processes.

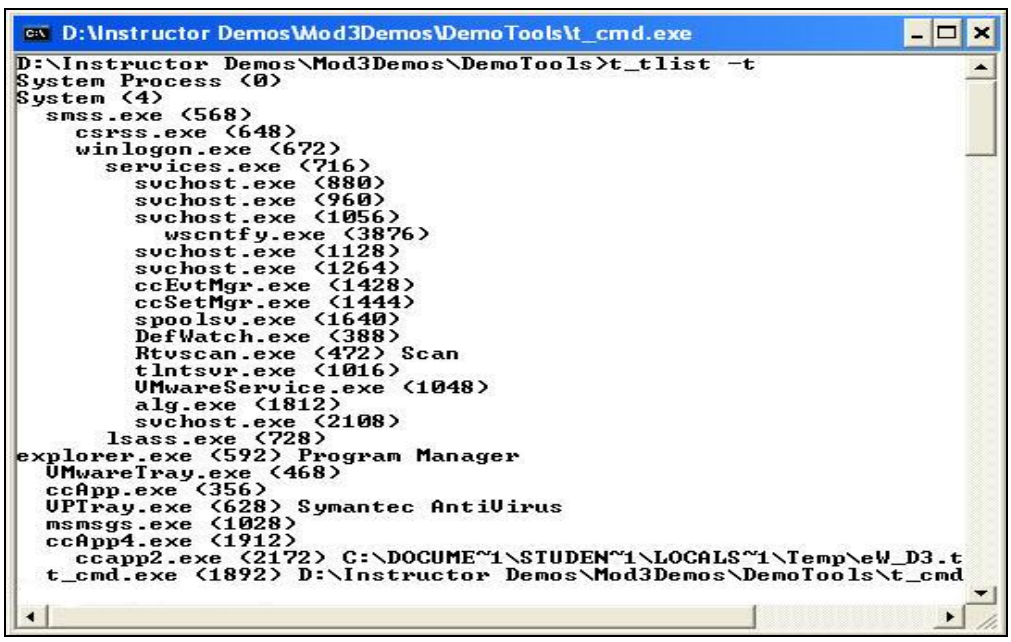

Figure 23: tlist.exe

\section{PsList (Windows)}

Sysinternals' PsList combines outputs from pmon and pstat to show process CPU and memory information (i.e., thread statistics). PsList, unlike pmon and pstat, allows you to view process and thread statistics on a remote computer.

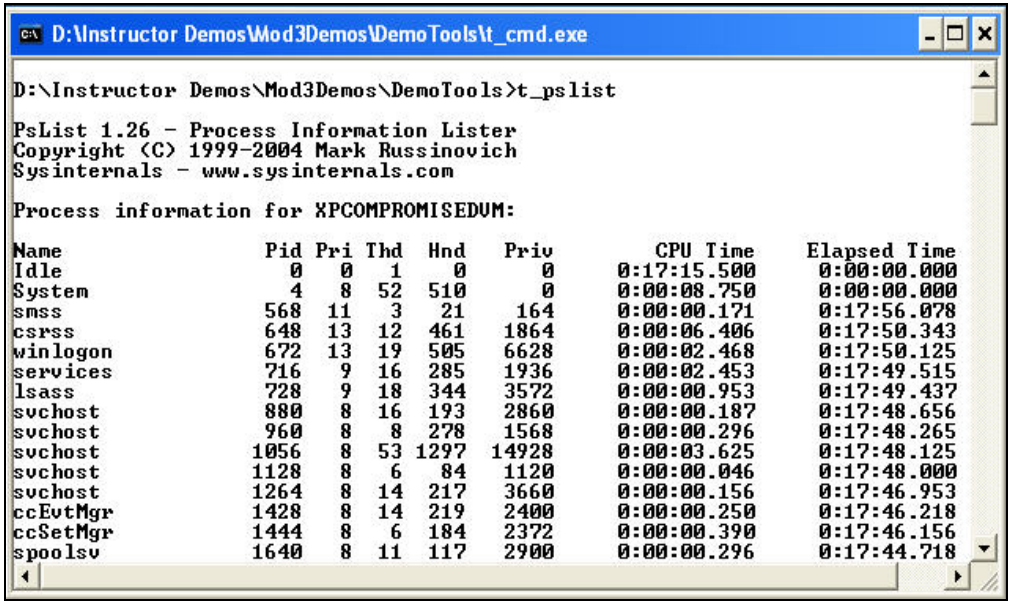

Figure 24: PsList 
Table 2 shows definitions for the PsList utilities’ command output headings.

Table 2: PsList Output Headings

$\begin{array}{llll}\text { Pri: } & \text { Priority } & \text { WS: } & \text { Working set } \\ \text { Thd: } & \text { Number of threads } & \text { WSPk: } & \text { Working set peak } \\ \text { Hnd: } & \text { Number of handles } & \text { Priv: } & \text { Private memory } \\ \text { Mem: } & \text { Memory usage } & \text { Faults: } & \text { Page faults } \\ \text { VM: } & \text { Virtual memory } & \text { NonP: } & \text { Non-paged memory }\end{array}$

\section{Memory Dump}

To learn more about a suspected rogue process, create a process memory dump using the pmdump.exe utility and then perform string searches on the file (e.g., use the Ctrl F command or the strings utility). Figure 25 shows the command string required to create a dump of the running process called winlogin.

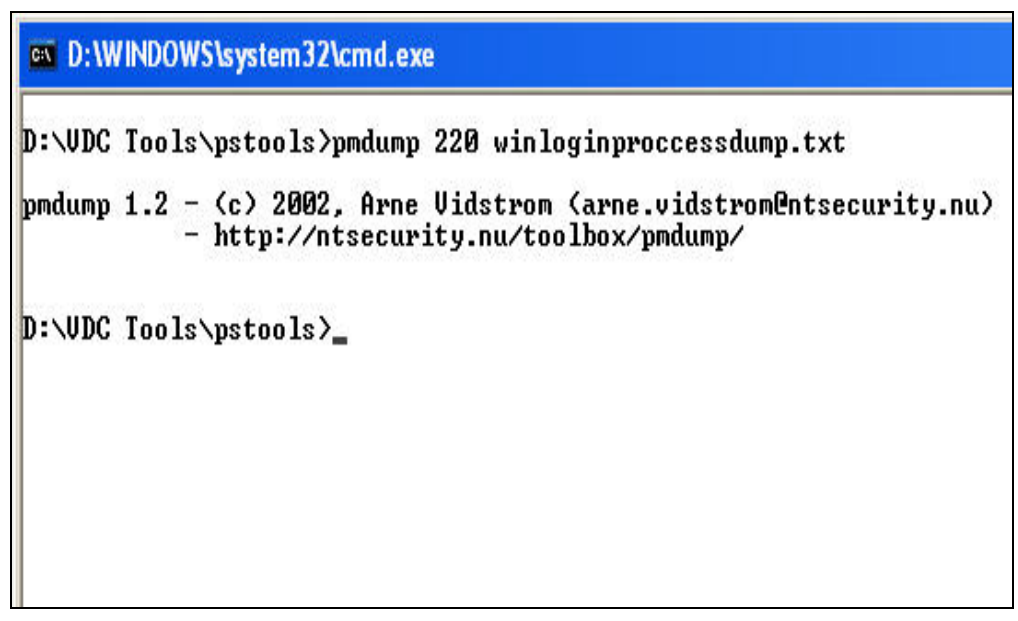

Figure 25: A Process Memory Dump

A better alternative is to export the running process' executable image to a forensic tool testbed for closer analysis.

\section{Top (Linux)}

The top command provides an ongoing look at processor activity in real time. It lists the most CPU-intensive tasks and includes an interactive interface for sorting processes by CPU usage, memory usage and runtime. Sort features can also be specified in the personal or system-wide configuration files. 


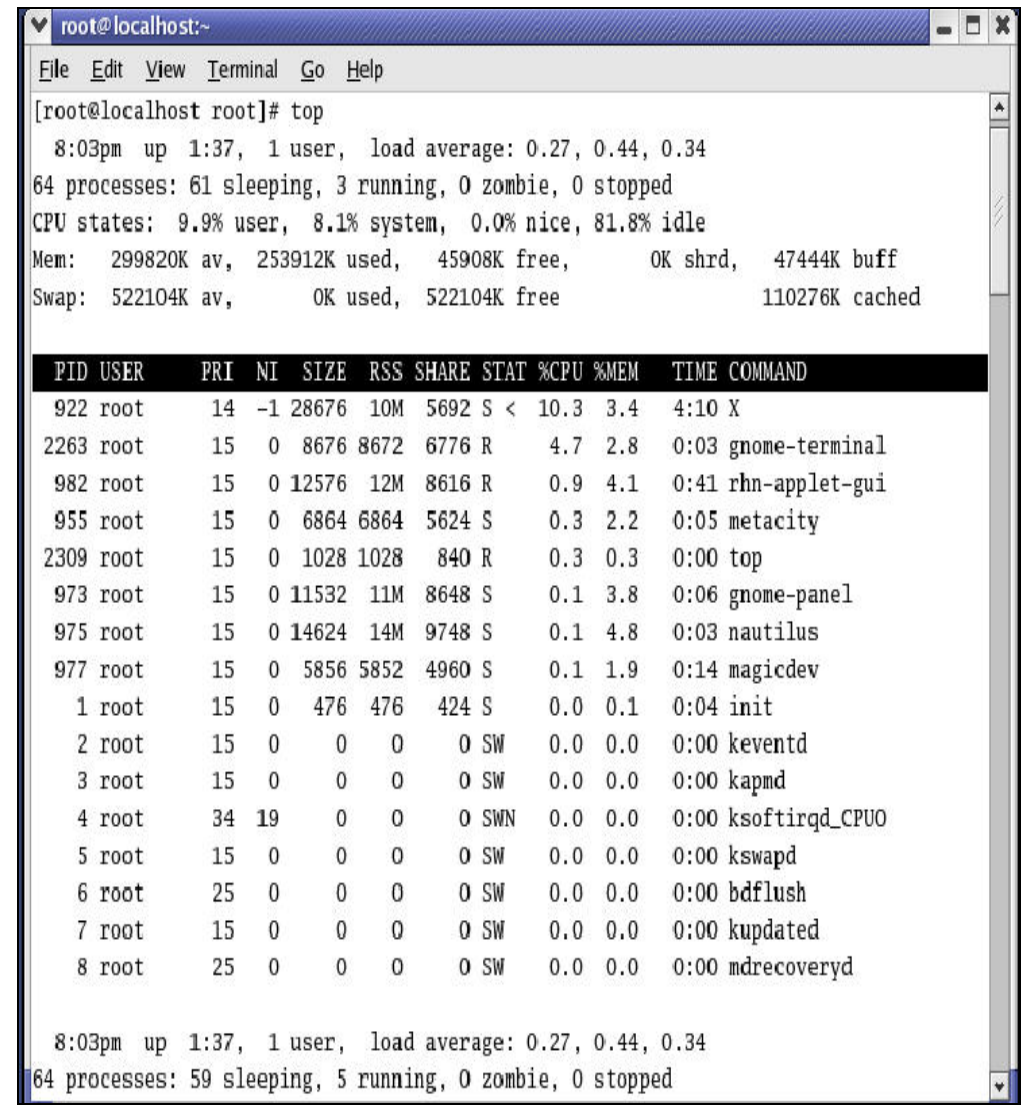

\section{Figure 26: The top Command}

Be aware that top can be configured to refresh at regular intervals so that results created immediately after each other may be different.

While top is running, press the appropriate key to update speed (s), hide idle processes (i), or kill a processes $(\mathrm{k})$.

\section{$W$ (Linux)}

The $w$ command displays the current processes for each shell of each user.

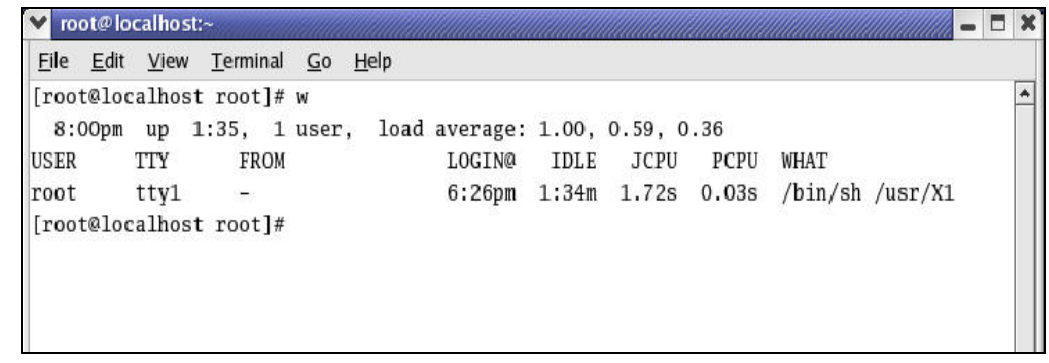

Figure 27: The w Command 
Output includes the following fields [Mandia 01]:

Table 3: w Command Output Fields

\begin{tabular}{|l|l|}
\hline Field & Description \\
\hline TTY & $\begin{array}{l}\text { Control terminal assigned to the users session. A ttyn (where } n \text { is zero or a positive integer) } \\
\text { signifies a logon at the console (a user logging on to the system from the local console or } \\
\text { keyboard). A ptsn or ttypn may signify a connection over the network. }\end{array}$ \\
\hline FROM & $\begin{array}{l}\text { Fully qualified domain name or numerical IP address of the remote host. A hyphen (-) in } \\
\text { this field corresponds to a local logon at console. }\end{array}$ \\
\hline LOGIN@ & Local starting time of the connection \\
\hline IDLE & Length of time since the last process was run \\
\hline JCPU & Time used by all processes attached to the tty or pts \\
\hline PCPU & Processor time used by the current process in the WHAT column \\
\hline WHAT & Process that the user is currently running \\
\hline
\end{tabular}

\section{Ps (Linux)}

The ps command, along with the netstat command, is one of the most powerful Linux administrative management commands.

\begin{tabular}{|c|c|c|c|c|c|c|c|}
\hline \multicolumn{8}{|c|}{ [rootQLinux_Compromised root]\# ps -aux } \\
\hline USER & PID \%CPU & \%MEM & USZ & RSS TTY & STA & START & TIME COMMAND \\
\hline root & 14.5 & 0.2 & 1512 & $512 ?$ & S & $22: 46$ & $0: 06$ init \\
\hline root & 20.0 & 0.8 & $\theta$ & 0? & SW & $22: 46$ & 0:08 [keventd] \\
\hline root & 30.0 & 0.0 & $\theta$ & 0? & SW & $22: 46$ & 0:00 [kapmd] \\
\hline root & 40.0 & 0.8 & $\theta$ & 0? & SWN & $22: 46$ & $0: \theta 0$ [ksoftirqd/o] \\
\hline root & 70.0 & 0.8 & $\theta$ & 0? & SW & $22: 46$ & $0: 00$ [bdf lush] \\
\hline
\end{tabular}

\section{Figure 28: The ps Command}

Figure 28 displays information about the root's currently running processes. A subset of the ps command's 80 options that are most useful to first responders are listed in Table 4 [Barrett 04].

Table 4: A Subset of ps Options

\begin{tabular}{|l|l|}
\hline Option & Description \\
\hline \$ ps -ux & View current processes \\
\hline \$ ps -U user & View other system users running processes \\
\hline \$ ps -C program_name & View all occurrences of a program \\
\hline \$ ps -p4, 8, 2203 & View selected processes 4, 8, 2203 \\
\hline \$ ps -efww & View all processes with full command lines \\
\hline
\end{tabular}


Table 5 describes some output headings for ps and top output [Nemeth 01].

Table 5: $\quad$ Output Headings for ps and top

\begin{tabular}{|c|c|}
\hline Field & Description \\
\hline USER & Username of the processes owner \\
\hline PID & Process ID \\
\hline$\% \mathrm{CPU}$ & Percentage of the CPU this process is using \\
\hline \%MEM & Percentage of real memory this process is using \\
\hline VSZ & Virtual size of the process, in kilobytes \\
\hline RSS & \\
\hline $\mathrm{TT}$ & \\
\hline STAT & $\begin{array}{l}\text { Current Process Status } \\
\mathrm{R}=\text { Runnable } \\
\mathrm{I}=\text { Sleeping }(<20 \mathrm{sec}) \quad \mathrm{D}=\text { In disk wait } \\
\mathrm{T}=\text { Stopped } \\
\text { Additional Flags } \\
\mathrm{L}=\text { Some pages are locked in core (for rawio) } \\
\mathrm{S}=\text { Process is a session leader (head of control terminal) } \\
\mathrm{W}=\text { Process is swapped out } \\
+=\text { Process is in the foreground of its control terminal }\end{array}$ \\
\hline START & Time the process was started \\
\hline TIME & CPU time the process has consumed \\
\hline COMMAND & Command name and arguments \\
\hline
\end{tabular}




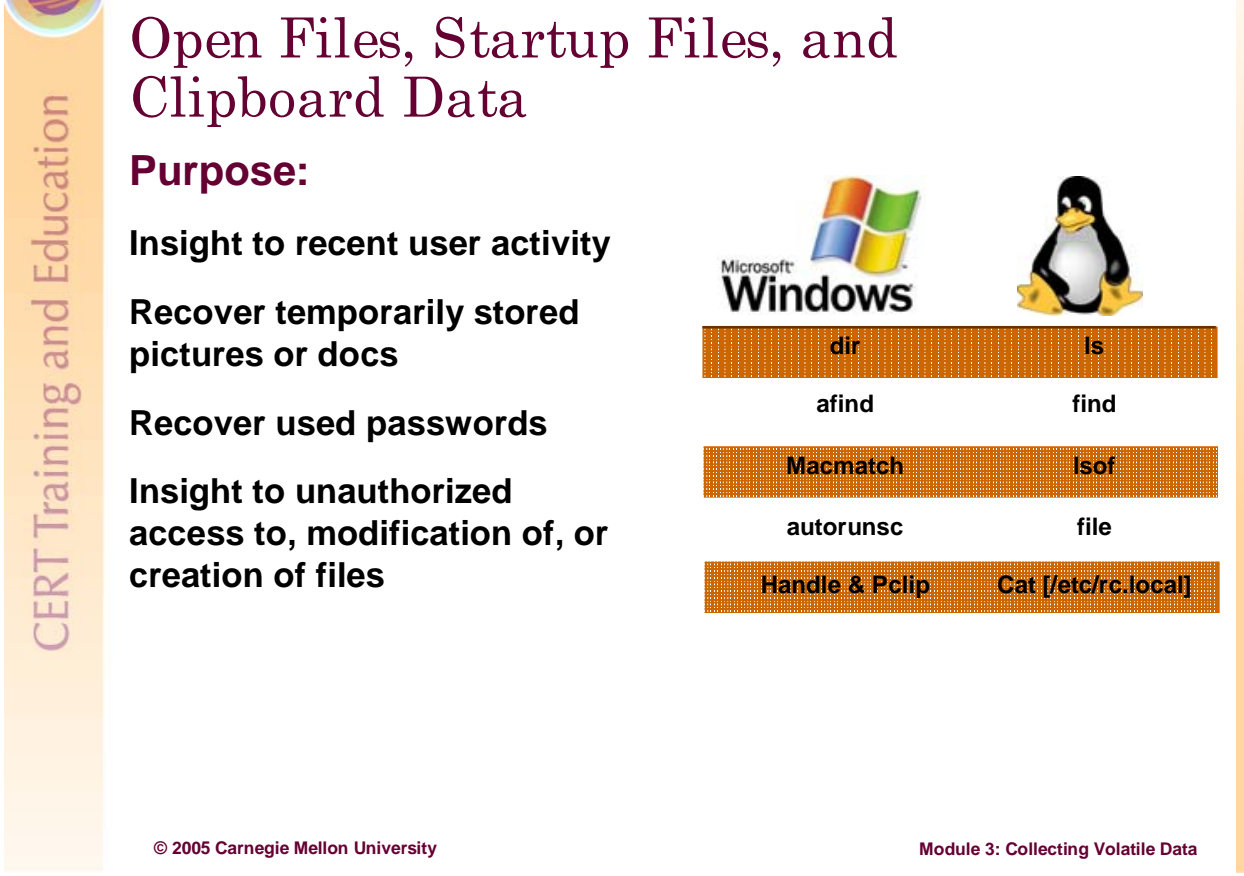

\subsubsection{Open Files, Startup Files, and Clipboard Data}

When malware such as a Trojan or Rootkit infects a system, files are created that allow the malware to start (when the system is rebooted) or continue to run (when the user logs out). For example, such files are generally created with the startup folders for Windows computers or within the rc.local file for Linux.

Therefore, collect and document the following data:

- open or recently created documents

- $\quad$ startup files and programs

- Dates and Times associated with MAC times for critical folders and files like Windows System32 folder and Linux’s proc file

Examine this data as follows:

- Review open files for sensitive data or information that may be relevant to the security incident.

- Look for valuable data like passwords, images, and pieces of documents on the clipboard.

- Search for unusual MAC times on critical folders and startup files.

\section{Dir (Windows)}

The native dir command lists the last access times on files and folders. 
Figure 29 shows how to collect the last access times on all files and folders within the c: drive. With options, you can specify certain folders (e.g., System32).

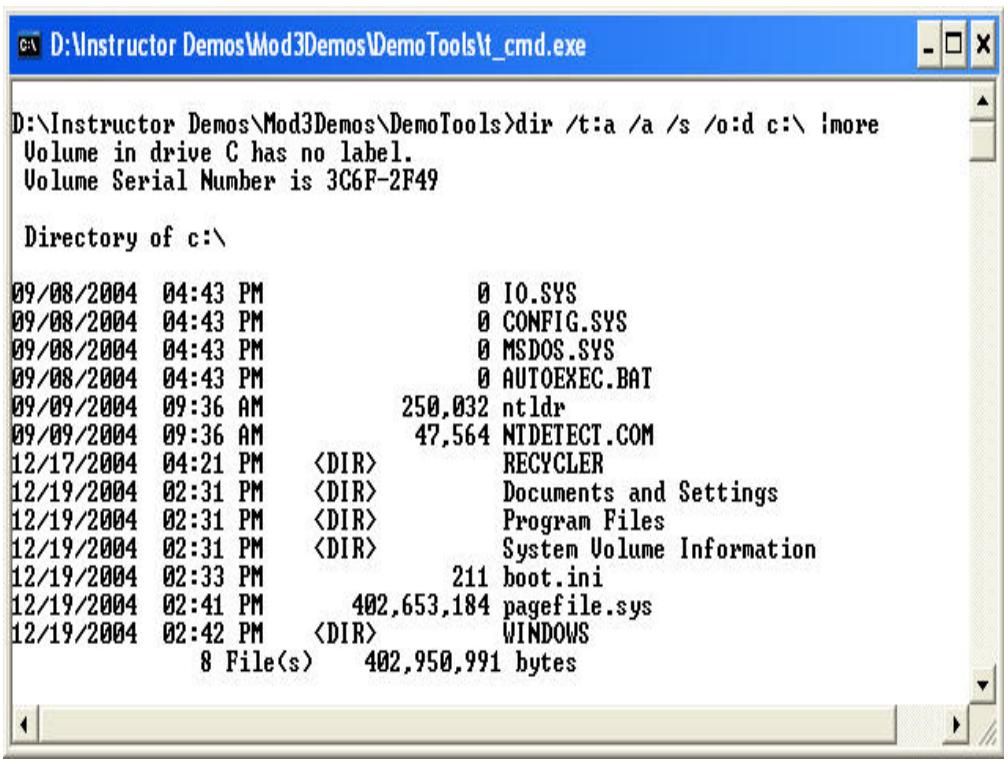

Figure 29: The dir Command

The native dir command includes the following options:

Table 6: dir Command Options

\begin{tabular}{|l|l|}
\hline Option & Description \\
\hline /t: a & Displays the last access field to be displayed and used for sorting \\
\hline$/ \mathrm{a}$ & Displays all files....Plus any hidden files \\
\hline$/ \mathrm{s}$ & Displays files in specified directory and all subdirectories \\
\hline /o:d & Displays files in sorted order by date \\
\hline
\end{tabular}




\section{Afind (Windows)}

Figure 30 shows how to search for a file's access time within a specified location.

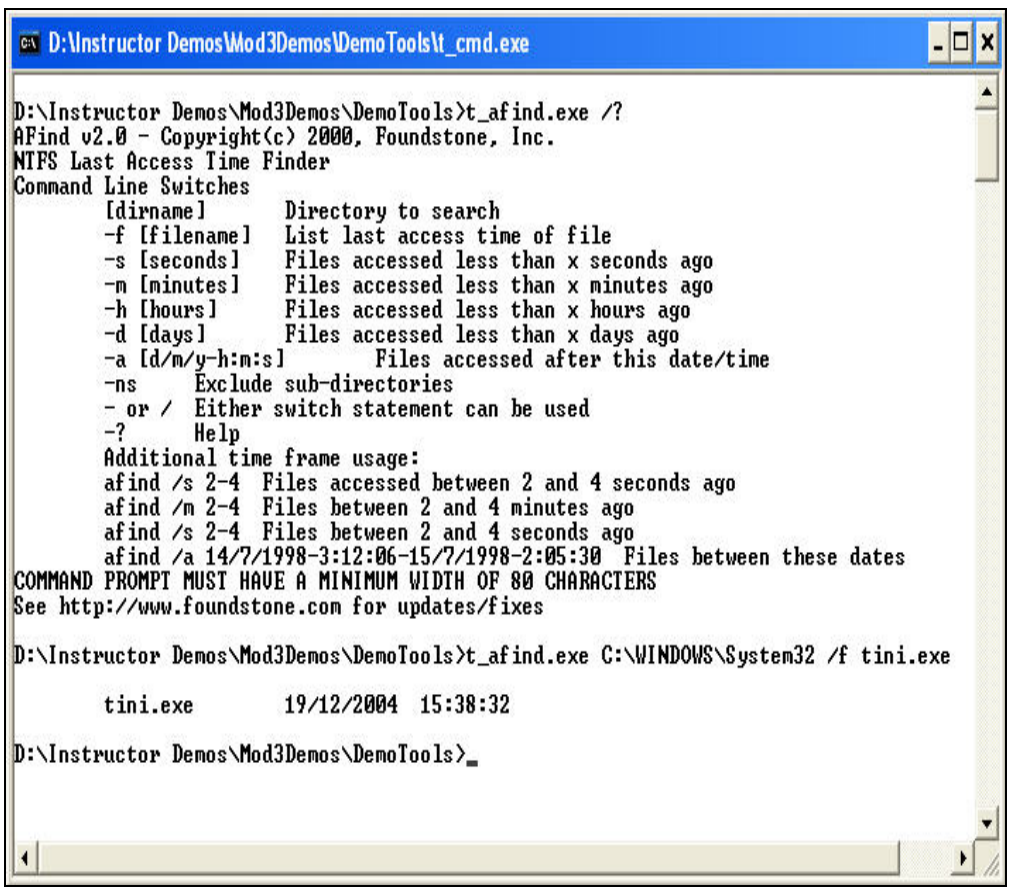

Figure 30: The afind Command

By specifying the general time period of the security incident, you can compare access times with logon information provided from ntlast and begin to determine user activity even if file logging had not been enabled.

\section{MACMatch (Windows)}

NTSecurity.nu-developed MACMatch allows you to search for files by their last write, last access, or creation time without changing any of these times [Vidstrom 04].

Usage is shown in Figure 31, which includes a search within a certain timeframe for recently accessed files such as the tini.exe. 


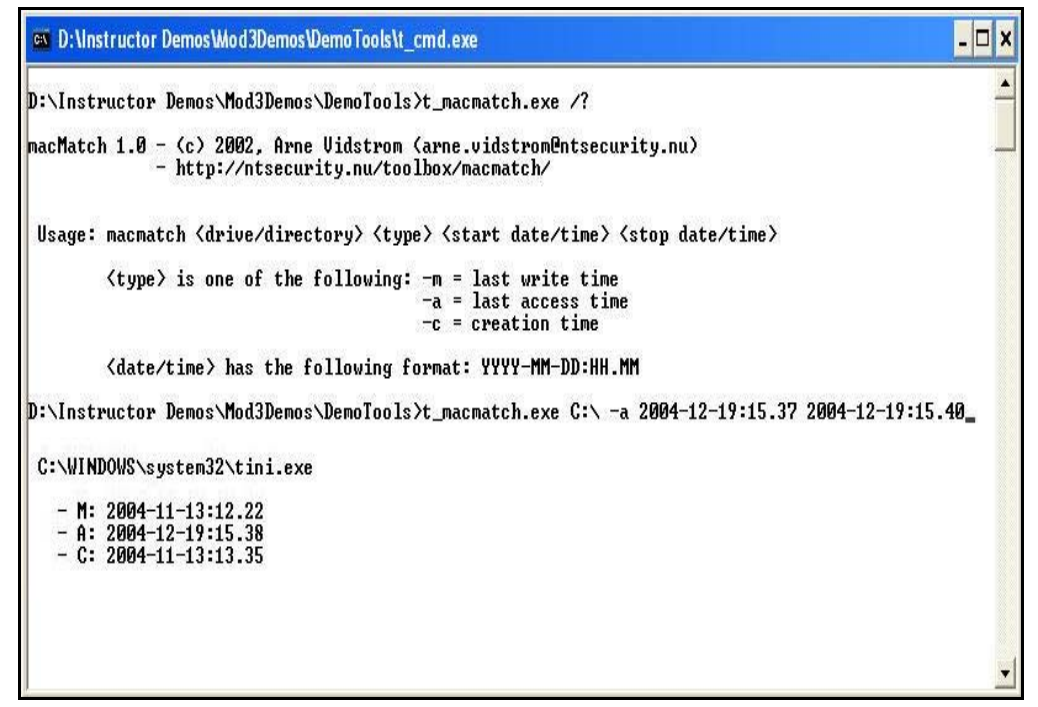

\section{Figure 31: MACMatch}

\section{Autorunsc (Windows)}

Sysinternals' Autoruns and DiamondCS' Autostart are freeware utilities that list files in the startup areas. Both incorporate a GUI, which is a drawback: Autorunsc is Autoruns command-line version. All three products provide much more detail than Windows' Msconfig utility.

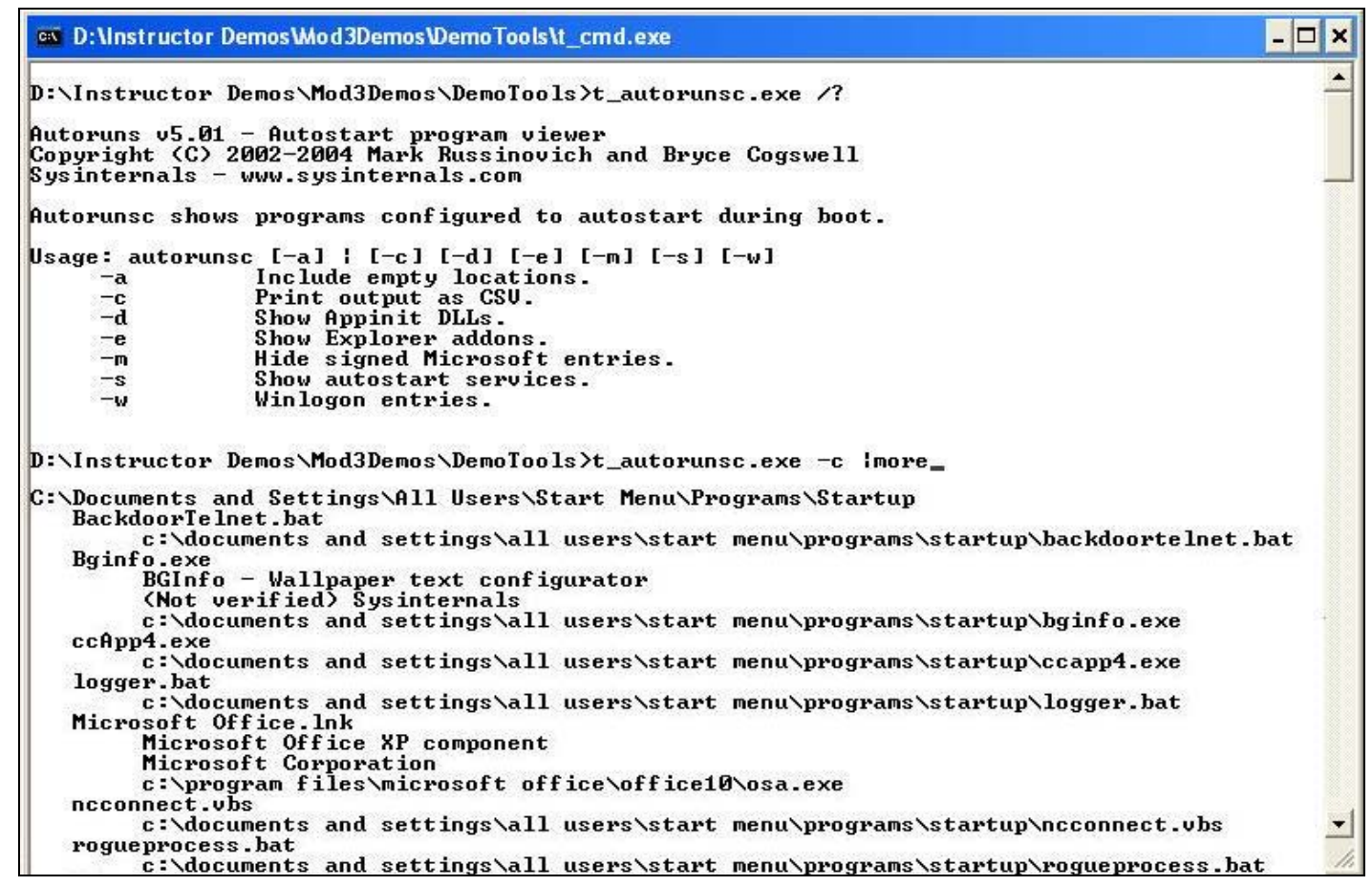

Figure 32: Autorunsc 
Autoruns has a comprehensive knowledge of auto-starting locations and shows what programs are configured to run during system bootup or login in the order Windows processes them. Programs include ones in your startup folder and Run, RunOnce, and other registry keys. You can configure Autoruns to show other locations, including Windows Explorer shell extensions, toolbars, browser helper objects, winlogon notifications, auto-start services, and much more.

\begin{abstract}
Autoruns' "Hide Signed Microsoft Entries" option helps you to zoom in on thirdparty auto-starting images that have been added to your system. Also included in the download package is a command-line equivalent that can output in CSV format, Autorunsc [Russinovich 04b].
\end{abstract}

\title{
PsFile and Handle (Windows)
}

Unsaved files or edits are lost after the system is shut down. Sysinternals' PsFile and Handle utilities allow you to display open files (executables and user-created), programs with open files, and the names of all program handles.

PsFile is a command-line utility that shows a list of files on a system that are opened remotely, and it also allows you to close opened files either by name or by a file identifier [Russinovich 01].

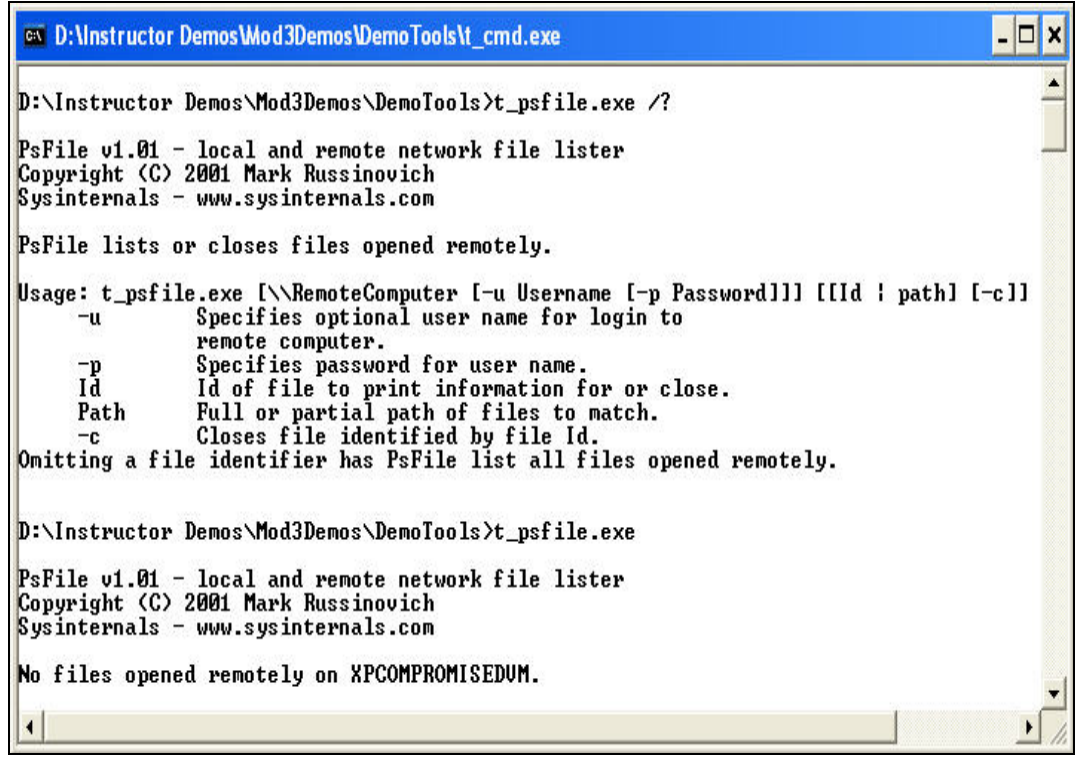

\section{Figure 33: PsFile}

Handle is a utility that displays information about open handles for any process in the system. You can use it to see the programs that have a file open, or to see the object types and names of all the handles of a program [Russinovich 04c]. 
In Figure 34 we demonstrate how we can utilize the handle.exe utility to look at all open handles for the potential rogue process svchost1.

\begin{tabular}{|c|c|c|c|}
\hline \multicolumn{2}{|c|}{ cir D:Instructor DemosWod3DemosiDemoToolsit_cmd.exe } & $-\square$ & $\mathbf{x}$ \\
\hline \multicolumn{2}{|c|}{$\begin{array}{l}\text { D: } \backslash \text { Instructor Demos } \backslash \text { Mod3Demos } \backslash \text { DemoTools }>t \text { handle }-a-p \text { suchost1 } \\
\text { Handle v2.2 } \\
\text { Copyright (C) 1997-2004 Mark Russinovich } \\
\text { Sysinternals - ww.sysinternals.com }\end{array}$} & & $\Delta$ \\
\hline \multirow{2}{*}{\multicolumn{2}{|c|}{ 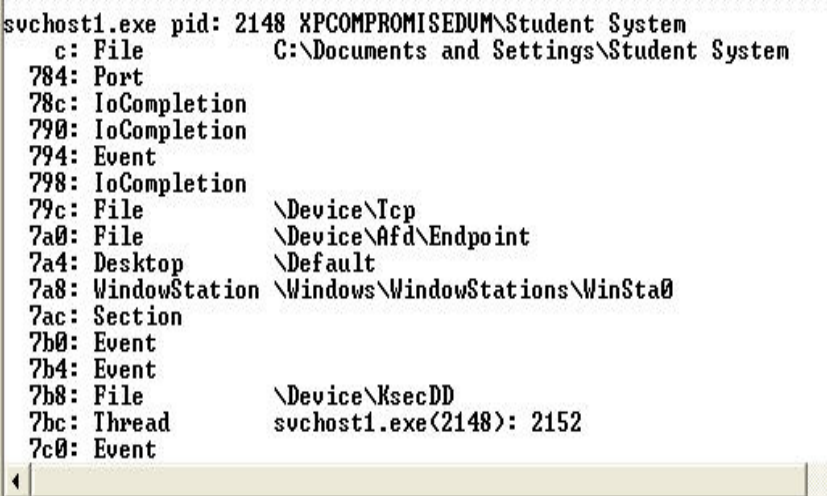 }} & & \\
\hline & & 1 & \\
\hline
\end{tabular}

Figure 34: Handle

\section{Pclip (Windows)}

Two freeware utilities, GNU's pclip utility and Harlan Carvey’s Perl script, allow you to dump the Windows clipboard contents: a document fragment, password, or image.

The clipboard is nothing more than an area of memory for temporarily holding information [Carvey 04].

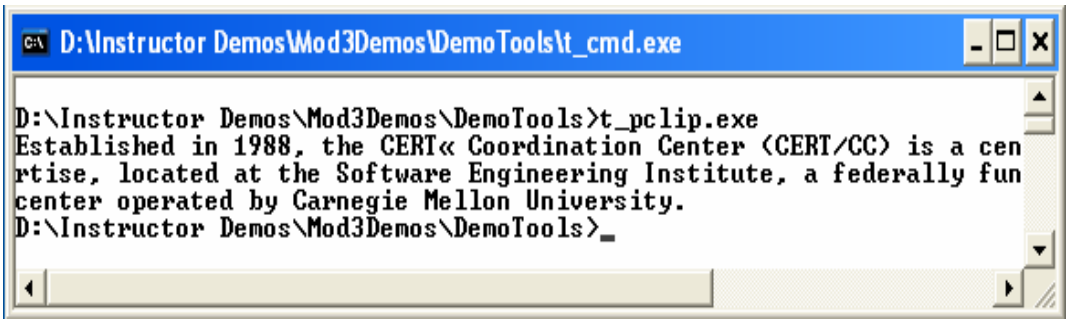

Figure 35: Pclip

In Figure 35, pclip displays a copied text string.

\section{Ls (Linux)}

The native Is command, like the Windows dir command, allows you to list files and their file access times. In Figure 36, ls -alRu displays the last access times for files in the root folder. 


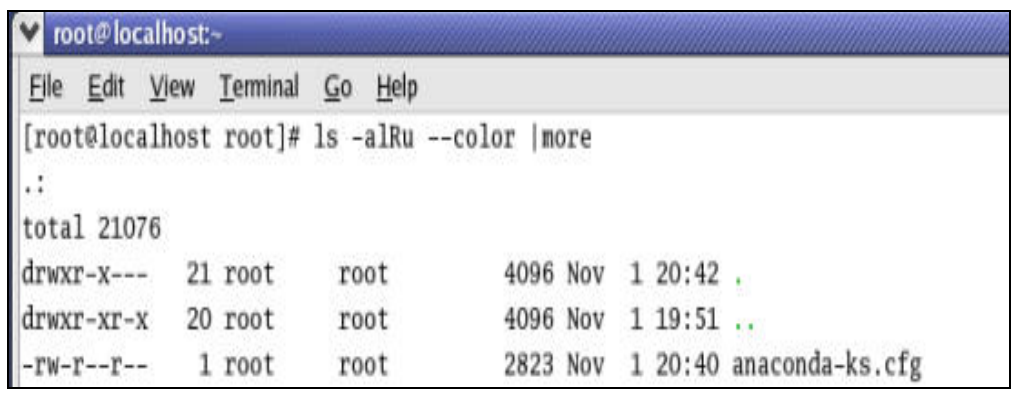

\section{Figure 36: The Is Command}

For Linux, three different permissions are tracked for every file and folder on the hard drive: read (r), write (w), and execute (x). To check file permissions for all the files in your current working directory, enter the following in a terminal window:

$$
\text { \# ls -la }
$$

A sample output is as follows [CAE 04]:

-rw-r--r-- 1 root root 2823 Nov 1 20:40

- The first character in the above line specifies whether the object is a file (-), a directory (d), or a link to another file or directory (l).

- The next group of three characters defines the read, write, and execute permissions (in that order) for the owner of the object.

- The next group of three characters describes the permissions for the group that controls the object.

- The final group describes the permissions for everyone else, usually called the global permissions.

- The owner of the object is given in the third column and the group the object belongs to is in the fourth column.

In Figure 36, root owns anaconda-ks.cfg and has read and write access. The group and everyone else can only read the file. Remember that root always has the ability to change the permissions on any file or directory.

To display all files sorted by the last modification time and date of each file, enter the following command:

$$
\text { ls }- \text { alct }
$$




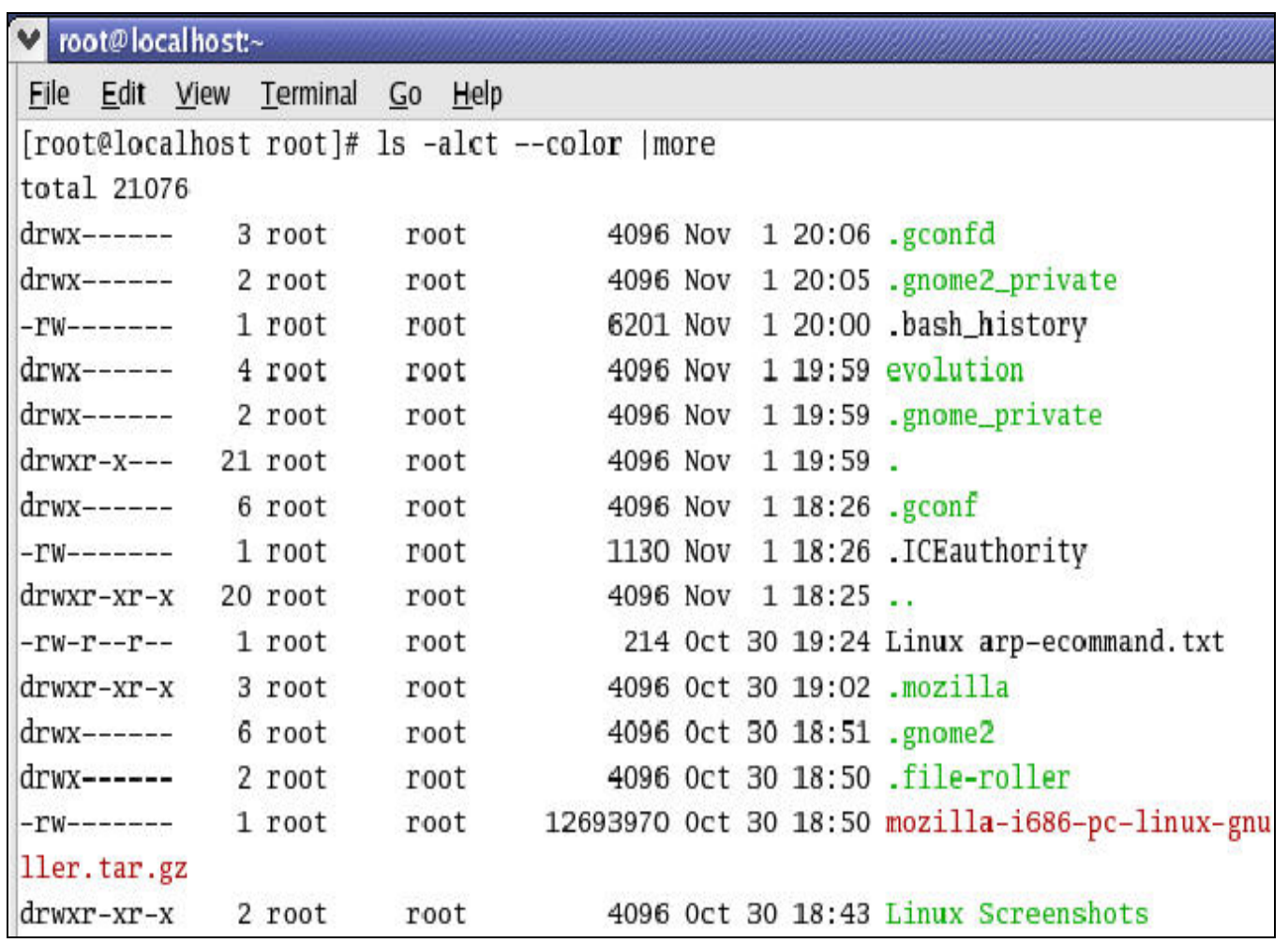

Figure 37: Using Is - alct to Show Last Modification Date and Time

Common ls parameters are listed in Table 7.

Table 7: Common Is Parameters

\begin{tabular}{|l|l|}
\hline Parameter & Function \\
\hline$-\mathrm{a}$ & Do not hide entries starting with a. \\
\hline$-\mathrm{l}$ & Use a long listing format. \\
\hline$-\mathrm{R}$ & List subdirectories recursively \\
\hline$-\mathrm{u}$ & Show last access time \\
\hline$-\mathrm{C}$ & Show last modification time \\
\hline$-\mathrm{t}$ & Sort list by time \\
\hline
\end{tabular}

\section{Lsof (Linux)}

The native Isof command allows you to list information about open files and the processes that might have launched them. You can discover, among other things, the command and user that opened the file as well as the file's size and PID.

The $+D$ [directory] parameter shown in Figure 38 forces 1 sof to perform a full-descent directory tree search for open files within a specific directory. 


\begin{tabular}{|c|c|c|c|c|}
\hline \multicolumn{5}{|c|}{\begin{tabular}{|l|l} 
root@localhost: \\
\end{tabular}} \\
\hline File Edit & View Iermi & nal $\underline{G}$ & Help & \\
\hline \multicolumn{5}{|c|}{ [root@localhost root]\# lsof +D /root } \\
\hline COMMAND & PID USER & FD & TYPE & EVICE SIZE NODE NAME \\
\hline bash & 864 root & cwd & DIR & $8,24096286849 /$ root \\
\hline startx & 910 root & cwd & DIR & $8,24096286849 /$ root \\
\hline xinit & 921 root & cwd & DIR & $8,24096286849 /$ root \\
\hline X & 922 root & cwd & DIR & $8,24096286849 /$ root \\
\hline gnome-ses & 925 root & cwd & DIR & $8,24096286849 /$ root \\
\hline
\end{tabular}

Figure 38: Isof +D

The $-i$ option displays all Internet and x.25 (HP-UX) network files.

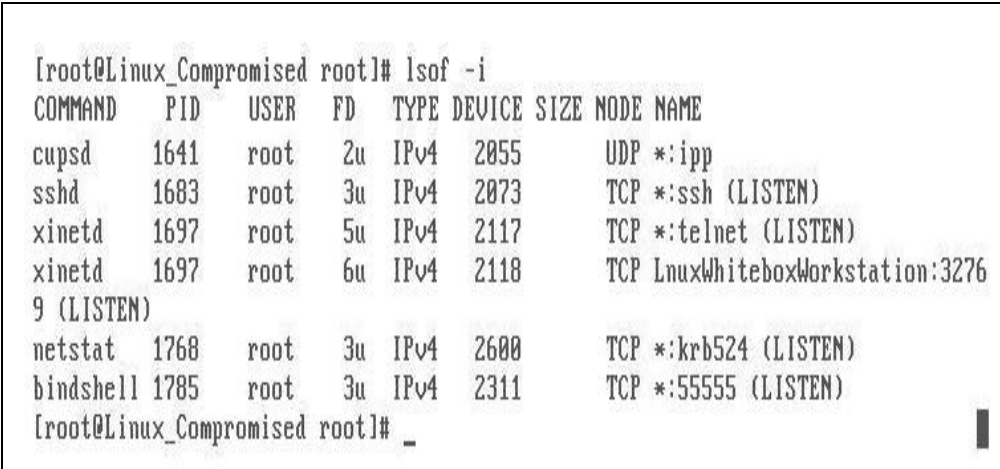

Figure 39: Isof -i

\section{Find and Locate (Linux)}

The find [directory] [option] [filename] command searches for particular filenames as well.

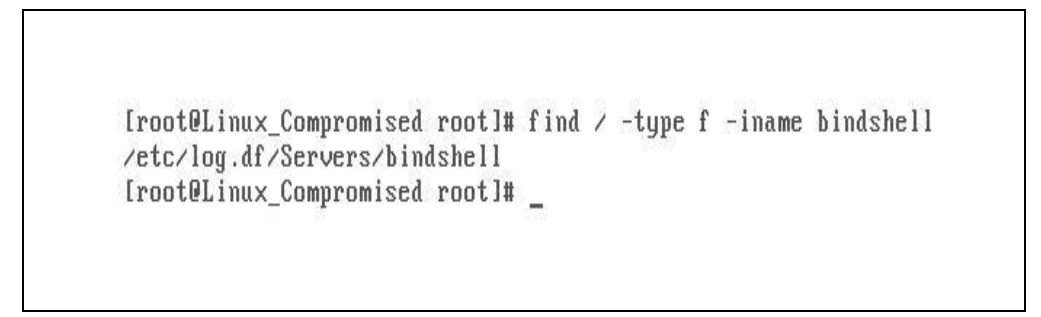

Figure 40: The find Command

The locate [filename] command searches the complete file system for references to the filename you specify. 
[rooteLinux_Compromised root]\# locate bindshell

/etc/log.df/Servers/bindshell.pl

/etc/log.df/Servers/bindshell.c

/etc/log.df/Servers/bindshell

[rooteLinux_Compromised root]\# _

Figure 41: The locate Command

\section{Unlinked Files (Linux)}

Unlinked files are marked for deletion when processes that access them terminate [Mandia 01]. Because they disappear when the system is powered down, you should attempt to recover them.

To display open files that have been unlinked and marked for deletion, use the lsof $+\mathrm{L} 1$ command as shown in Figure 42.

\begin{tabular}{|c|c|c|c|c|c|c|c|c|}
\hline File Edit & \multicolumn{8}{|c|}{ Frooteloc } \\
\hline \multicolumn{9}{|c|}{ [root@localhost root]\# lsof +L1 | more } \\
\hline COMMAND & PID & USER FD & TYPE & DEVICE & SIZE & NLINK & NODE & NAME \\
\hline $\mathrm{X}$ & 922 & root mem & DEL & 0,4 & & 0 & 393228 & /SYSV00000000 \\
\hline $\mathrm{X}$ & 922 & root mem & DEL & 0,4 & & 0 & 425997 & /SYSV00000000 \\
\hline $\mathrm{X}$ & 922 & root mem & DEL & 0,4 & & 0 & & /SYSV00000000 \\
\hline $\mathrm{X}$ & 922 & root mem & DEL & 0,4 & & 0 & 32769 & /SYSV00000000 \\
\hline $\mathrm{X}$ & 922 & root mem & DEL & 0,4 & & 0 & 65538 & /SYSV00000000 \\
\hline $\mathrm{x}$ & 922 & root mem & DEL & 0,4 & & 0 & 98307 & /SYSV00000000 \\
\hline $\mathrm{X}$ & 922 & root mem & DEL & 0,4 & & 0 & 131076 & /SYSV00000000 \\
\hline $\mathrm{X}$ & 922 & root mem & DEL & 0,4 & & 0 & 163845 & /SYSV00000000 \\
\hline $\mathrm{X}$ & 922 & root mem & DEL & 0,4 & & 0 & 196614 & /SYSV00000000 \\
\hline $\mathrm{X}$ & 922 & root mem & DEL & 0,4 & & 0 & 229383 & /SYSV00000000 \\
\hline $\mathrm{X}$ & 922 & root mem & DEL & 0,4 & & 0 & 262152 & /SYSV00000000 \\
\hline $\mathrm{X}$ & 922 & root mem & DEL & 0,4 & & 0 & 294921 & /SYSV00000000 \\
\hline $\mathrm{X}$ & 922 & root mem & DEL & 0,4 & & 0 & 3342346 & /SYSV00000000 \\
\hline $\mathrm{x}$ & 922 & root mem & DEL & 0,4 & & 0 & 360459 & /SYSV00000000 \\
\hline $\mathrm{x}$ & 922 & root mem & DEL & 0,4 & & 0 & 458766 & /SYSV00000000 \\
\hline $\mathrm{X}$ & 922 & root mem & DEL & 0,4 & & 0 & 3375119 & /SYSV00000000 \\
\hline $\mathrm{X}$ & 922 & root mem & DEL & 0,4 & & 0 & 3407888 & /SYSV00000000 \\
\hline $\mathrm{x}$ & 922 & root mem & DEL & 0,4 & & 0 & 1409046 & /SYSV00000000 \\
\hline $\mathrm{x}$ & 922 & root mem & DEL & 0,4 & & 0 & 1474583 & /SYSV00000000 \\
\hline $\mathrm{X}$ & 922 & root mem & DEL & 0,4 & & 0 & 1736729 & /SYSV00000000 \\
\hline gnome-ses & 925 & root mem & DEL & 0,4 & & 0 & & /SYSV00000000 \\
\hline gnome-ses & 925 & root mem & DEL & 0,4 & & 0 & 32769 & /SYSV00000000 \\
\hline metacity & 955 & root mem & DEL & 0,4 & & 0 & 393228 & /SYSV00000000 \\
\hline metacity & 955 & root mem & DEL & 0,4 & & 0 & 98307 & /SYSV00000000 \\
\hline
\end{tabular}

Figure 42: The Isof +L1 Command 
How UNIX deletes a file is best described by Prosise and Mandia [Mandia 01]:

When an attacker runs a process, he usually deletes the program file he executed from the file system in an effort to hide his actions. He is not truly deleting the program on the hard drive. The attacker is unlinking the file.

UNIX tracks a file's link count, which is a positive integer representing the number of processes currently using the file. When the link count equals zero, that means no process is using or needs the file, so it will be deleted. When an attacker deletes his rogue program, the program on the hard drive is removed from the directory chain (so it will not be displayed in an ls listing), the link count is decremented by one, and the file's deletion time is set. However, note that the link count does not equal zero until the process terminates.

Files marked for deletion (these are the unlinked files) at the time a system is powered down-whether gracefully (through normal shutdown procedures) or not (you pulled the power cord)-will ultimately end up deleted on the system. Let's examine why.

When UNIX mounts a file system, a "file system dirty" bit is set. When the operating system goes through a normal shutdown, every process is forced to close. The attacker's process terminates normally, and all file handles are closed. This means that the link count on the deleted file is set to zero. After all processes have exited and other general housekeeping items have been completed, the file system is un-mounted, and the file system dirty bit is cleared.

If the OS goes through a traumatic shutdown, the file system is left in an unstable state. Unlinked files may still have false link counts, and the dirty bit remains set. On the next boot-up, the file systems are mounted, and the operating system detects the nonzero value of the dirty bit. Most of the time, the administrator will be forced to wait while the system performs a file system check (fsck). The fsck utility will scan the entire file system for damage. If the utility comes across a file with a positive link count and a deletion time set, it will decrement the link count, rendering the file "deleted." Some versions of fsck will re-link the orphaned file to the lost+found directory, but this is not something that you can rely on.

\section{Chkconfig (Linux)}

The chkconfig -list command displays a list of startup services that will be run at the five different runlevels. This information may help you identify a rogue or Malware application that starts at one of the five runlevels. 


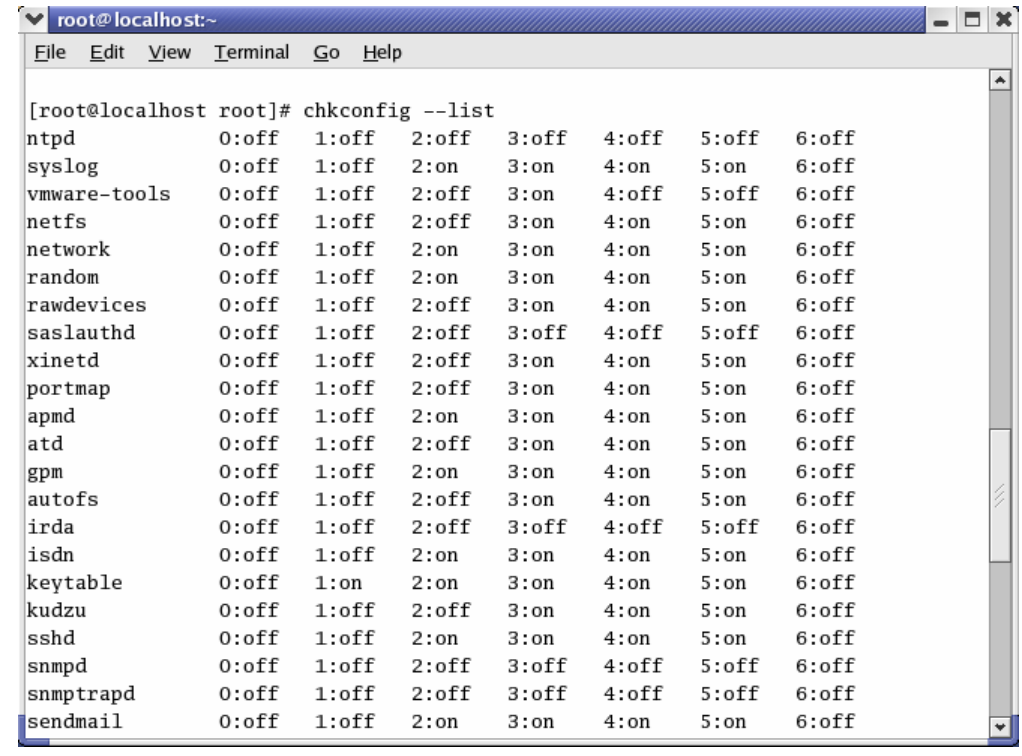

Figure 43: The chkconfig -list Command

chkconfig has five distinct functions: adding new services for management, removing services from management, listing the current startup information for services, changing the startup information for services, and checking the startup state of a particular service.

When chkconfig is run without any options, it displays usage information. If only a service name is given, it checks to see if the service is configured to be started in the current runlevel. If it is, chkconfig returns true; otherwise it returns false. The --level option may be used to have chkconfig query an alternative runlevel rather than the current one [Haas 04].

\section{Inittab File (Linux)}

The inittab file displays and describes the five runlevels. It describes which processes are started at bootup and during normal operation. Valid runlevels are 0-6 and as we can see in Figure 44, it is set to initdefault setting of 3. 


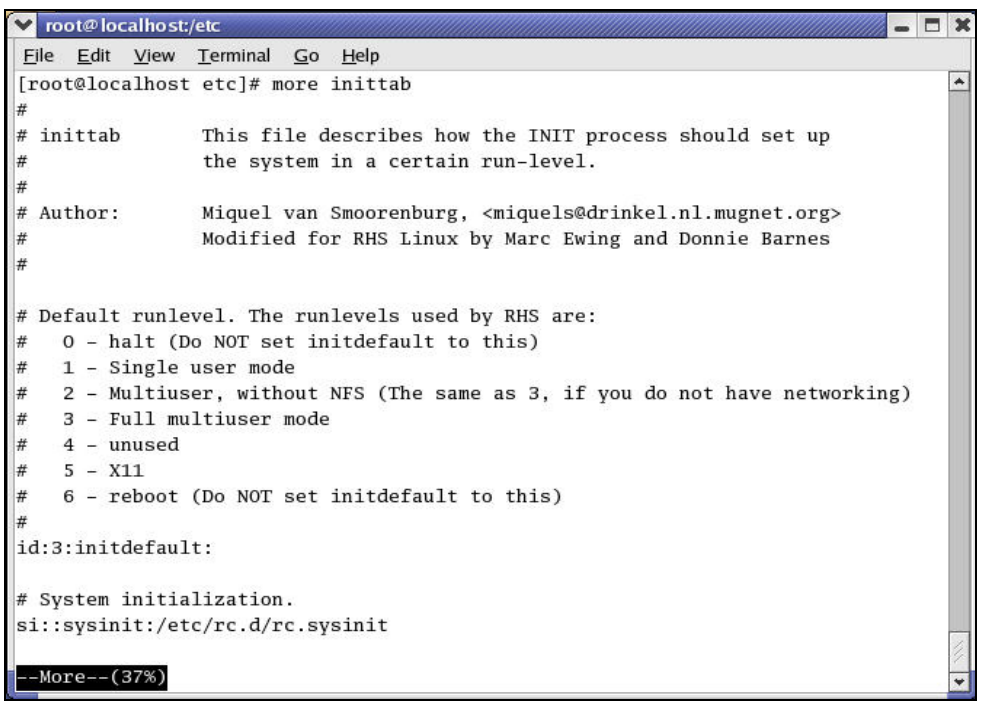

Figure 44: The Inittab File

\begin{tabular}{|llllllll|}
\hline sshd & $0:$ off & $1:$ off & $2:$ on & $3:$ on & $4:$ on & $5:$ on & $6:$ off \\
\hline
\end{tabular}

In the above example, the sshd service is off at the halt, single user, and reboot runlevels and on at multiuser without NFS, full mulituser, unused, and X11 runlevels.

\section{Cron Logs (Linux)}

In addition to startup services and open applications and files, collect the currently scheduled tasks. Attackers often schedule a malicious file to execute periodically so that the malware remains existent. The cron feature allows system administrators to schedule programs for future execution. All executed cron jobs are logged, usually in the /var/cron/log or in the default logging directory in a file called cron.

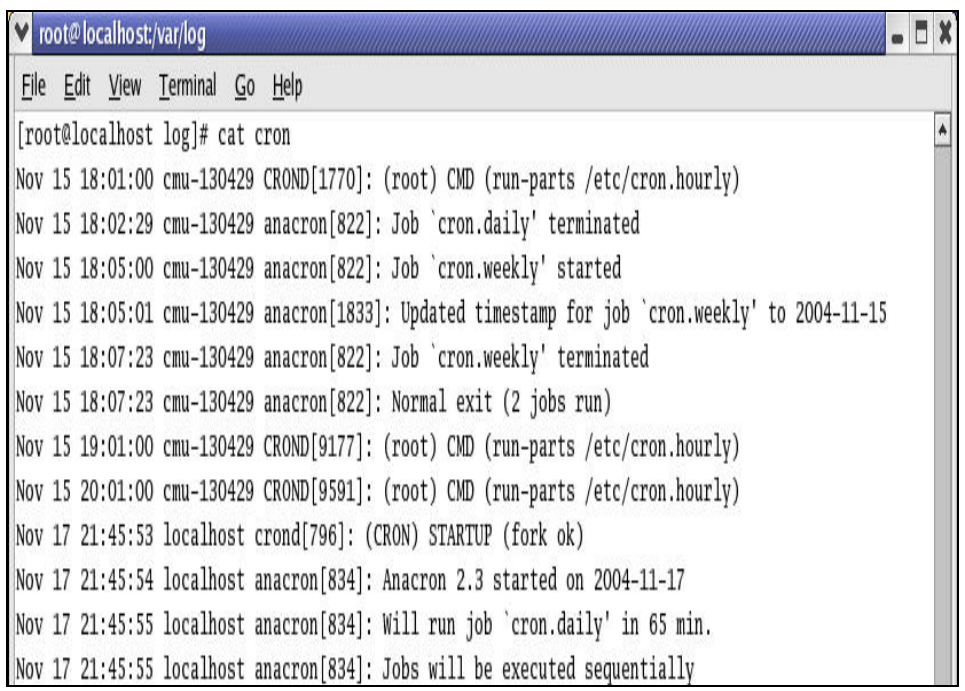

\section{Figure 45: A Cron Log}




\section{Logged On Users}

Purpose:

To determine the total number of authorized users that have

access to the system

To determine the access times of local and remotely connected users

What to look for:

Recently added user accounts

Escalation of user account privileges

\section{Local and remote user} accounts

๑) 2005 Carnegie Mellon University

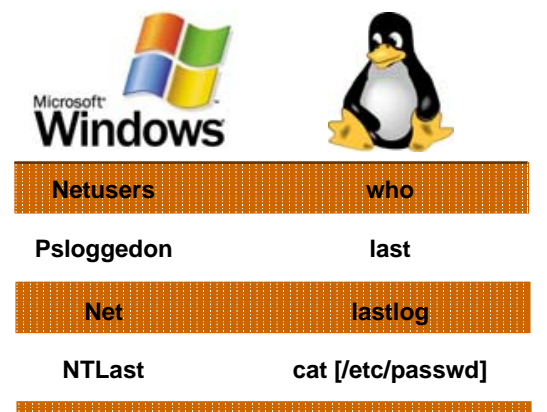

DumpUsers

cat [letc/shadow]

Module 3: Collecting Volatile Data

\subsubsection{Logged On Users}

The tools in this section help you identify logged on users. When you approach a suspicious computer, at least one user is generally logged on locally and others may be logged on remotely. Pay attention to the following:

- recently added user accounts

- escalated privileges for existing user accounts

- remote access accounts added to the system (remote users generally access the system through a shared drive, folder, backdoor, or printer)

- the total number of local and remote users that have access to the system or are currently logged onto the system

- times that the users logged on to the system, logged off, and the period during which they remained logged onto the system 


\section{Netusers (Windows: Local Users)}

SomarSoft's Netusers, a freeware command-line utility, allows you to display locally logged on users. It can display current interactive users or all users who have ever logged on through the computer [SystemTools 04].

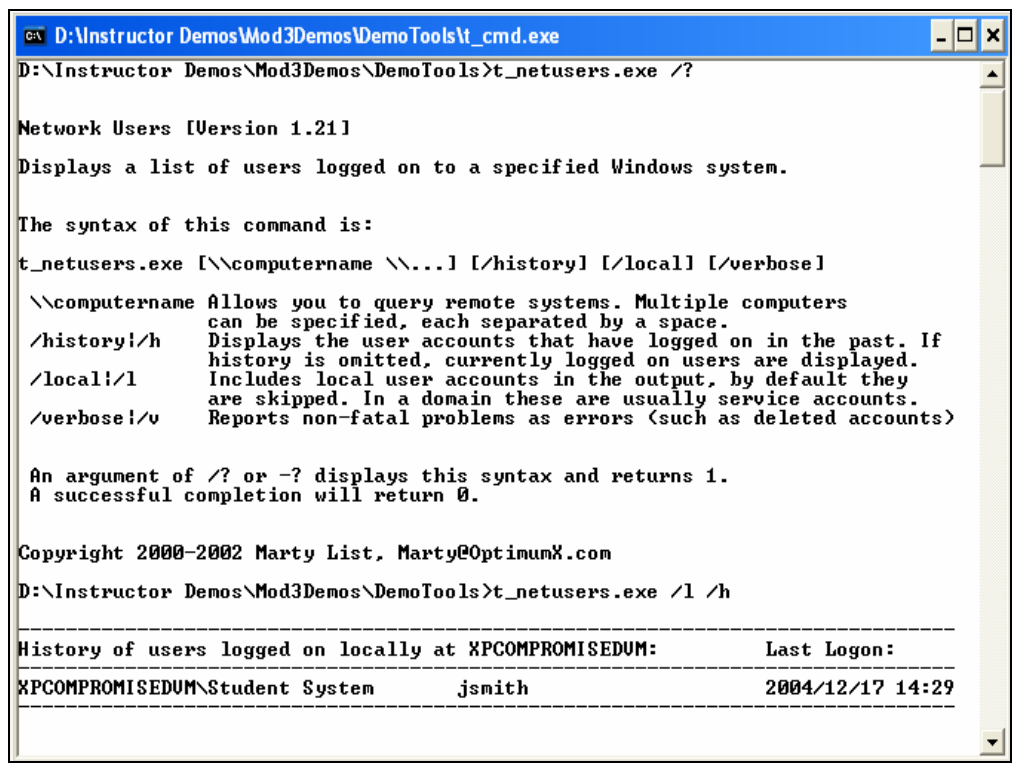

Figure 46: Netusers

\section{PsLoggedOn (Windows: Local and Remote Users)}

Sysinternals' PsLoggedOn, a freeware utility, allows you to display locally and remotely logged on users. In Figure 47, the machine was logged on to at 2:30 p.m. and no user is logged on via resource shares.

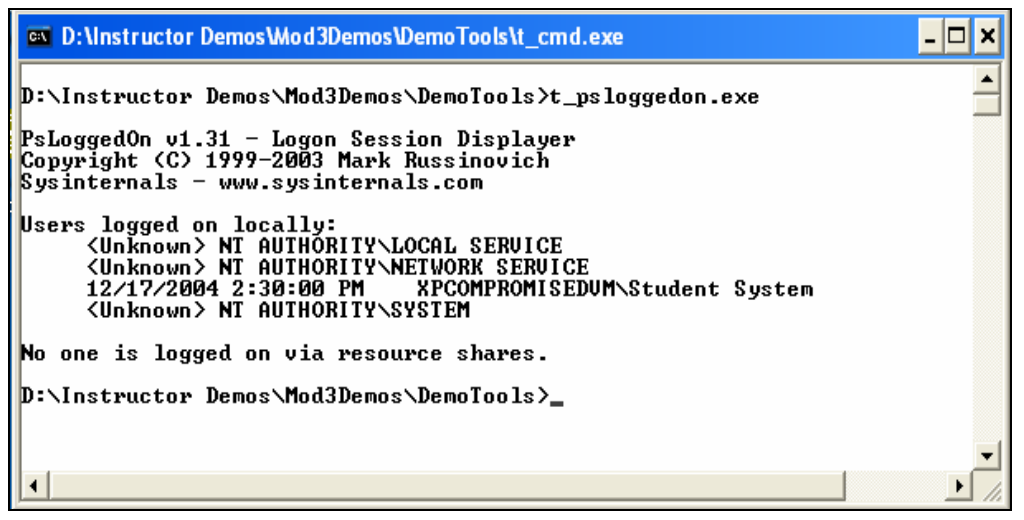

Figure 47: PsLoggedOn 


\section{Net (Windows: Local and Remote Users)}

The native net [user] command displays all local and remote users. Generally, system administrators use net user to create and modify user accounts on computers. If they use the command without command-line switches, all user accounts for the computer are displayed.

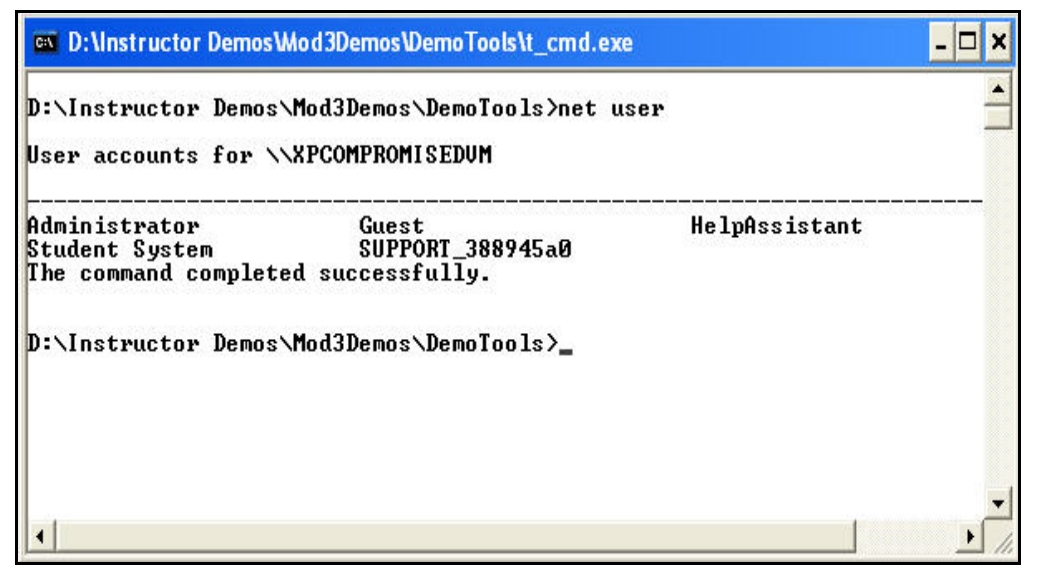

Figure 48: The net user Command

\section{Net (Windows: Remote Users)}

The native net [session] command displays information about remote connections established with the suspicious computer. For each connection, it displays the remote computer's name, IP address, client type, and idle time.

\section{NTLast (Windows: Remote Users)}

The NTLast utility from Foundstone can be used to collect event logs associated with logon attempts on the suspicious computer.

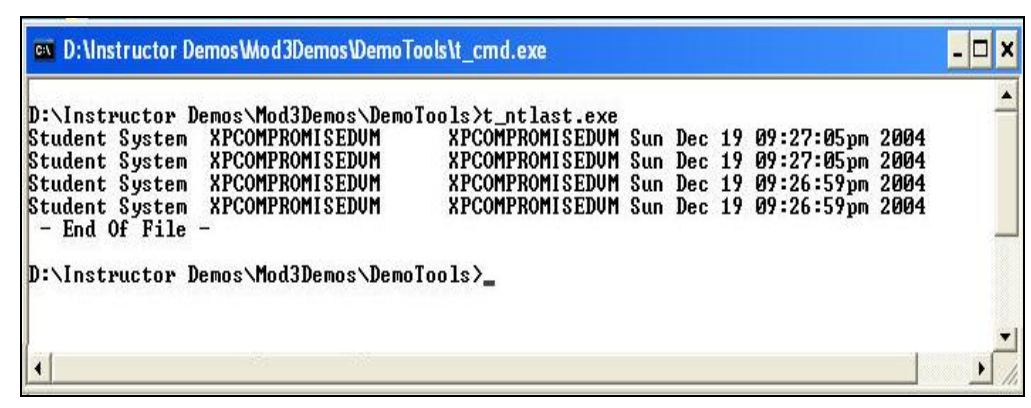

Figure 49: NTLast

For NTLast to work, the audit log feature had to have been enabled through the suspicious computer's Local Security Policy. If it is not, you will not get any information. 


\section{DumpUsers (Windows: Local Users)}

NTSecurity.nu's DumpUsers utility dumps account names and information even though RestrictAnonymous has been set to 1 .

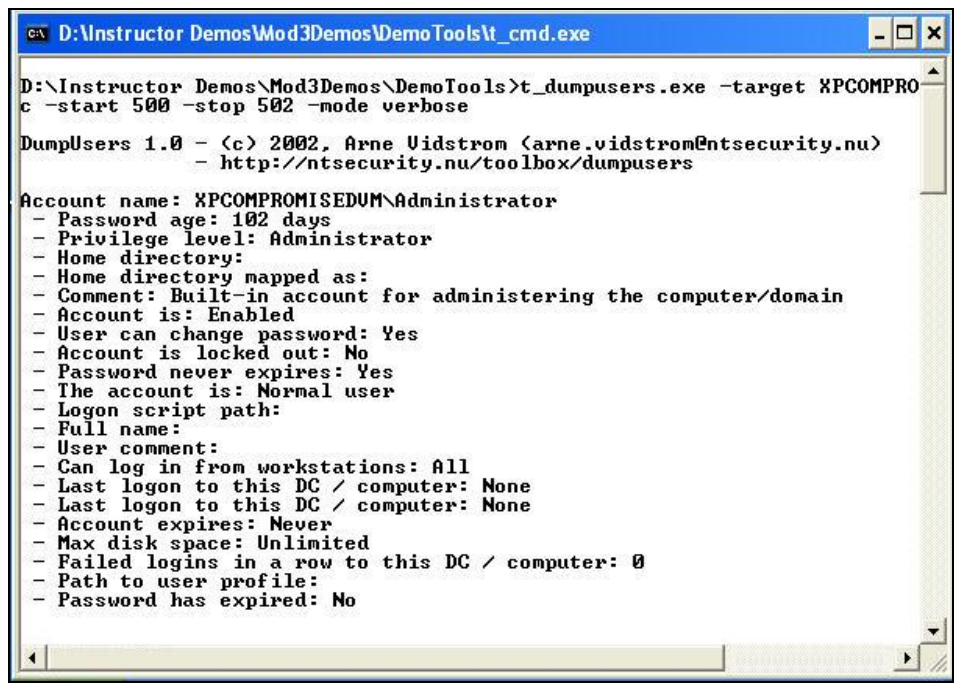

Figure 50: DumpUsers

The command line options selected in Figure 50 are as follows:

- Target (suspicious computer): XPCOMPROMISEDVM

- Type: notdc (not a domain controller)

- Start: 500 (the RID from which to start collecting information)

- Stop: 502 (the RID from which to stop)

- Mode: verbose

\section{Who (Linux: Local Users)}

The native who command displays the user that is currently logged on locally. With no options, who lists the name of each user currently logged in with their terminal, the time they logged on, and the name of the host from which they have logged in. Supply an optional system file (default is /etc/utmp) to obtain additional information.

\section{Who Am I, Who -uH (Linux: Local Users)}

The native who am $i$ command lets you quickly determine the currently logged on user. The who $-u H$ command displays the idle times for logged on user(s). Idle times can be good indicators of a logged on user's previous activity. 


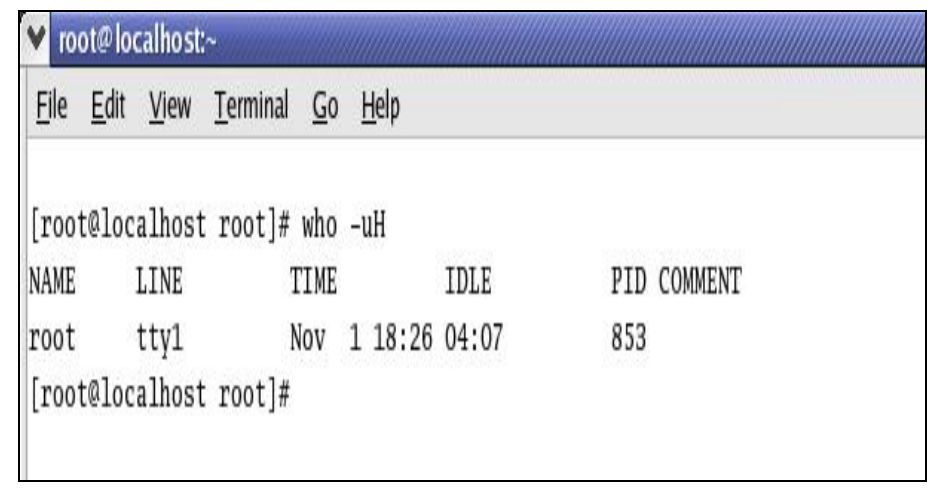

Figure 51: The who -uH Command

The native who $-q$ command displays all logged in user names and the number of logged in users. Without any parameters, the command displays the time of the last system boot and users currently logged on. It also prints any dead processes, system login processes, active processes spawned by init, the current run level, and the last system clock change.

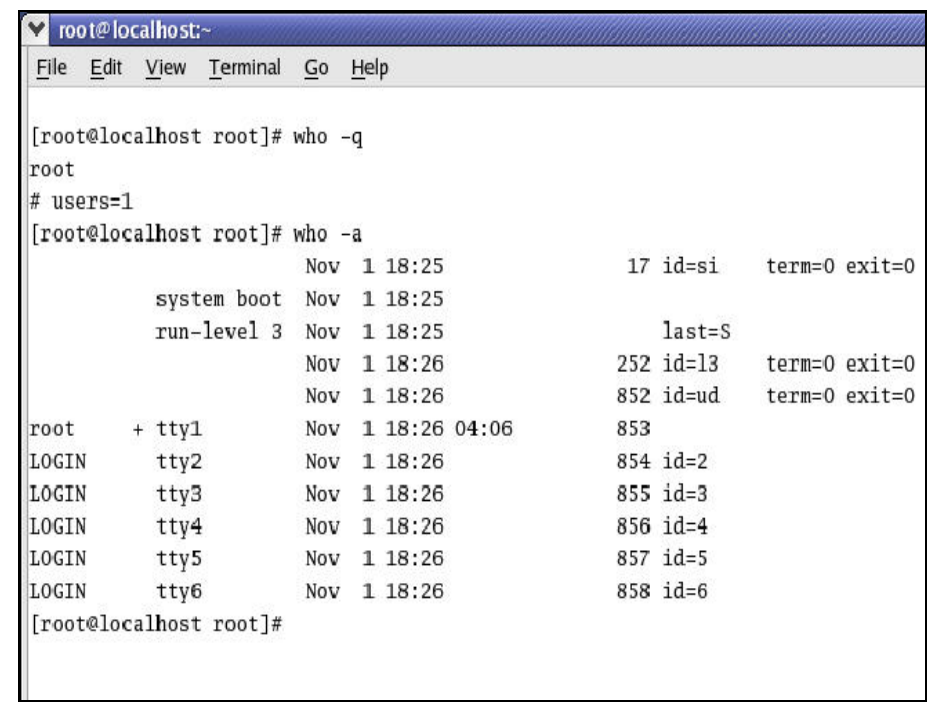

Figure 52: The who -q Command

Who -all-a (Linux: Local and Remote Users)

The native who - all or who - a commands display all currently logged on users, local and remote. 


\section{Last (Linux: Local and Remote Users)}

The native last command displays a history of logged on users, local and remote. By default, recent logins from the $/ \mathrm{var} / \mathrm{log} / \mathrm{wtmp}$ file are included. Specify a tty number or username for user- or terminal-specific output.

\begin{tabular}{|c|c|c|}
\hline 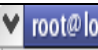 & & \\
\hline File E Edit & Ierminal Go Help & \\
\hline [rootaloc & root]\# last & \\
\hline wtmp beg. & Nov 1 19:31:39 & \\
\hline [rootaloc & root]\# lastlog & \\
\hline Username & Port From & Latest \\
\hline & tty1 & Mon Nov 1 18:26:44 -0500 2004 \\
\hline bin & & *Never logged in** \\
\hline daemon & & **Never logged in** \\
\hline adm & & *Never logged in** \\
\hline Ip & & *Never logged in** \\
\hline sync & & **Never logged in** \\
\hline shutdown & & *Never logged in** \\
\hline halt & & *Never logged in** \\
\hline mail & & *Never logged in** \\
\hline news & & *Never logged in** \\
\hline uucp & & *Never logged in** \\
\hline operator & & *Never logged in** \\
\hline
\end{tabular}

Figure 53: The last Command

\section{Lastlog (Linux: Local and Remote Users)}

The native lastlog command displays the last login times for system accounts. Login information is read from the /var/log/lastlog file.

\section{W (Linux: Local and Remote Users)}

The native $w$ command displays summaries of system usage, currently logged on users, and logged on user activities. This command is a combination of uptime, who, and ps -a. Specify a user for user-specific details. 


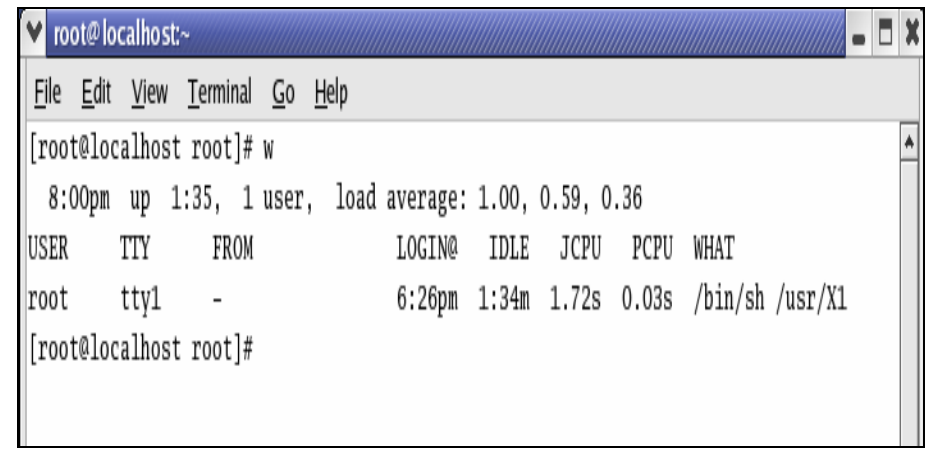

Figure 54: The w Command

\section{Passwd (Linux: Local and Remote Users)}

The /etc/passwd text file contains user account information, including one-way encrypted passwords. To display the file, use the cat /etc/passwd command.

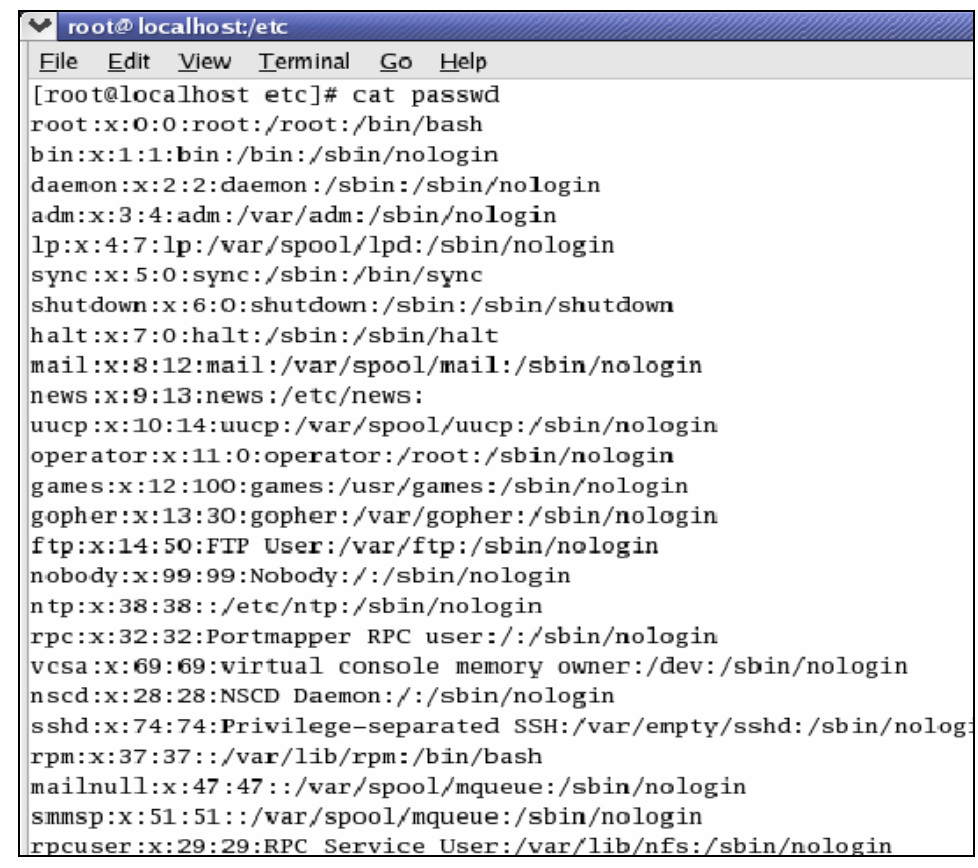

Figure 55: The cat /etc/passwd Command

\section{Shadow (Linux: Local and Remote Users)}

The /etc/shadow text file contains password and optional password aging information and is readable only by the root user. 


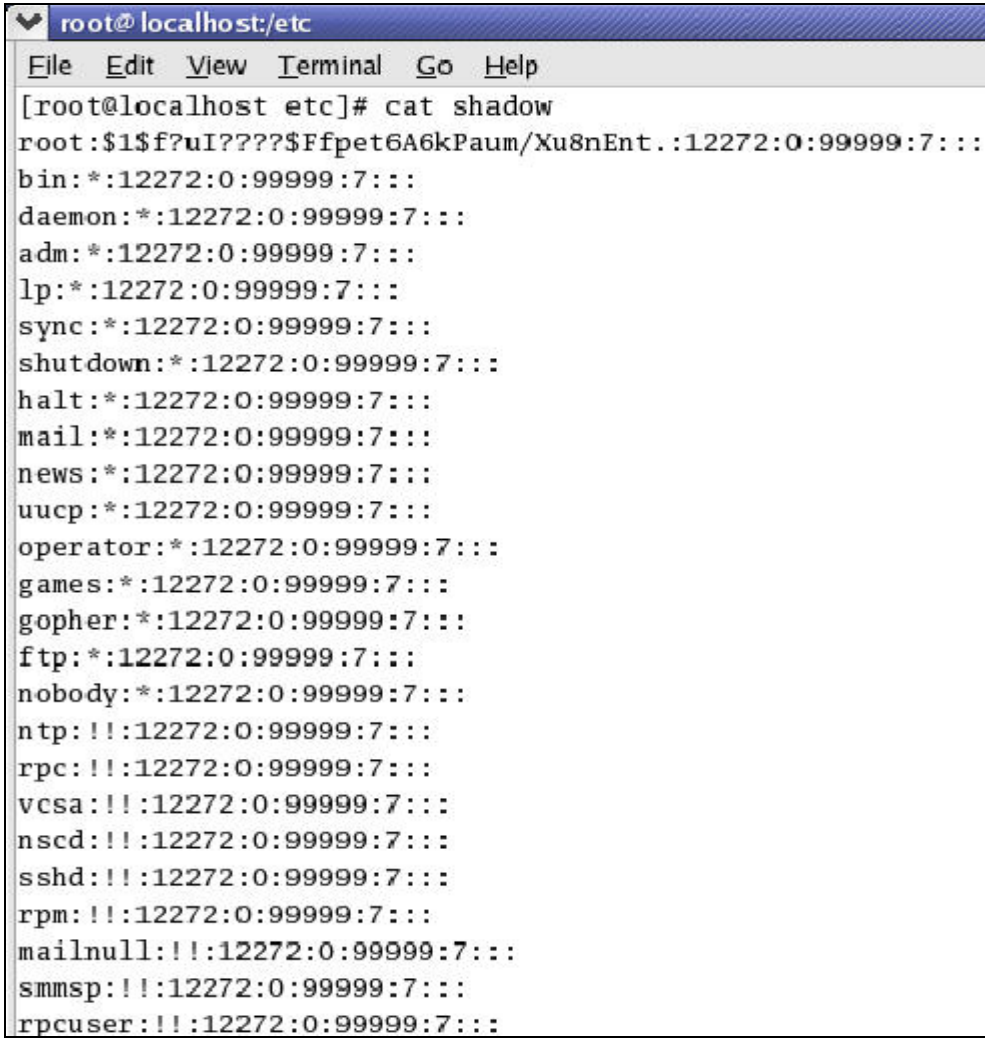

Figure 56: The cat shadow Command

As in the /etc/passwd file, each user's information is on a separate line. Each line has nine fields, delineated by colons:

- username

- encrypted password

- date password last changed

- number of days before password can be changed

- number of days before password can be changed

- number of days before password change is required

- number of days warning before password change

- number of days before the account is disabled

- date since the account has been disabled 


\section{DLLs or Shared Libraries}

\section{Purpose:}

\section{To determine possible rogue or modified DLLs and shared libraries}

What to look for:

Versions

Unfamiliar filenames

Recently added DLLs

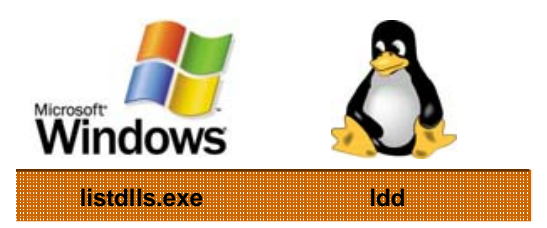

listdlls.exe

\subsubsection{DLLs or Shared Libraries}

A DLL (or shared library) file contains functions that are compiled, linked, and saved separately from the processes that use them. Functions in DLLs can be used by more than one running process. The OS maps the DLLs into the process' address space when the process is started or while it is running.

The tools in this section help you identify currently loaded DLLs or shared libraries so that you can examine them and determine if any are rogue. Filenames for rogue DLLs may be unidentified or unfamiliar (best case) or they may be masked so that they appear to be legitimate even though they have been corrupted or trigger malicious activity when linked.

As you use the tools in this section to identify DLLs, pay attention to the following:

- $\quad$ processes that are using the running DLLs

- unfamiliar DLLs

- compare the list of DLLs for a process with the expected DLLs for that process (check for number of DLLs and their dates, which should be the OS install date unless patches and hot fixes have been applied) 


\section{ListDLLs (Windows)}

Sysinternals' ListDLLs freeware utility displays all loaded DLLs with their version numbers, associates each loaded DLL with its application or executable, and flags loaded DLLs that have different version numbers than their corresponding on-disk files.

In Figure 57, ListDLLs is run without options to produce a list of DLLs.

\begin{tabular}{|c|c|c|}
\hline \multicolumn{3}{|c|}{ Gi D:IWINDOWSlsystem $32 \mathrm{lcm}$ d.exe } \\
\hline \multirow{2}{*}{\multicolumn{3}{|c|}{$\begin{array}{l}D=\backslash U D C \text { Tools }>\text { cd ListDLLs } \\
D=\backslash U D C \text { Tools } \backslash \text { List tDLLs }>1 \text { istdlls .exe } / ?\end{array}$}} \\
\hline & & \\
\hline \multicolumn{3}{|c|}{ 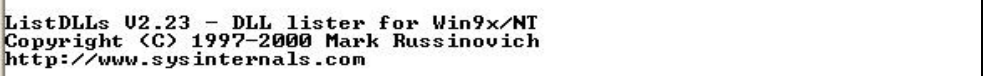 } \\
\hline \multirow{3}{*}{\multicolumn{3}{|c|}{ 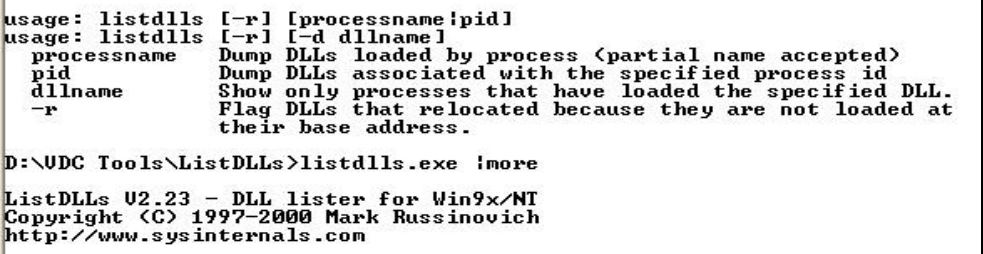 }} \\
\hline & & \\
\hline & & \\
\hline \multicolumn{3}{|c|}{$\begin{array}{l}\text { System pid: } 4 \text { <no command line> } \\
\text { Command line: }\end{array}$} \\
\hline \multicolumn{3}{|c|}{$\begin{array}{l}\text { smss .exe pid : } 2028 \\
\text { Command line : } \backslash \text { SystemRoot } \backslash S y s t e m 32 \backslash \text { smss .exe }\end{array}$} \\
\hline 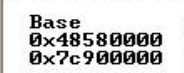 & $\begin{array}{ll}\text { Size } & \text { Uersion } \\
\text { Gxfgg日 } & \\
\text { Oxbดg日g } & 5.01 .2600 .2180\end{array}$ & 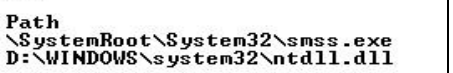 \\
\hline \multicolumn{3}{|c|}{ 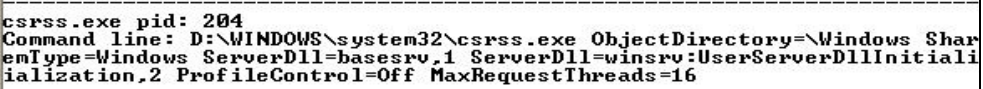 } \\
\hline 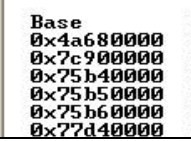 & 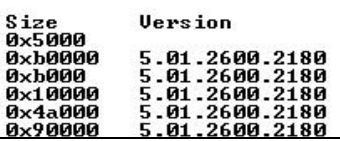 & 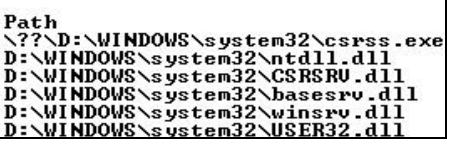 \\
\hline
\end{tabular}

\section{Figure 57: Using ListDLLs Without Options to Produce a List of DLLs}

In Figure 58, ListDLLs is used to examine a suspected rogue process (svchost1.exe). In comparing svchost1.exe with the legitimate svchost.exe, significant differences in the types and numbers of loaded DLLs are found.

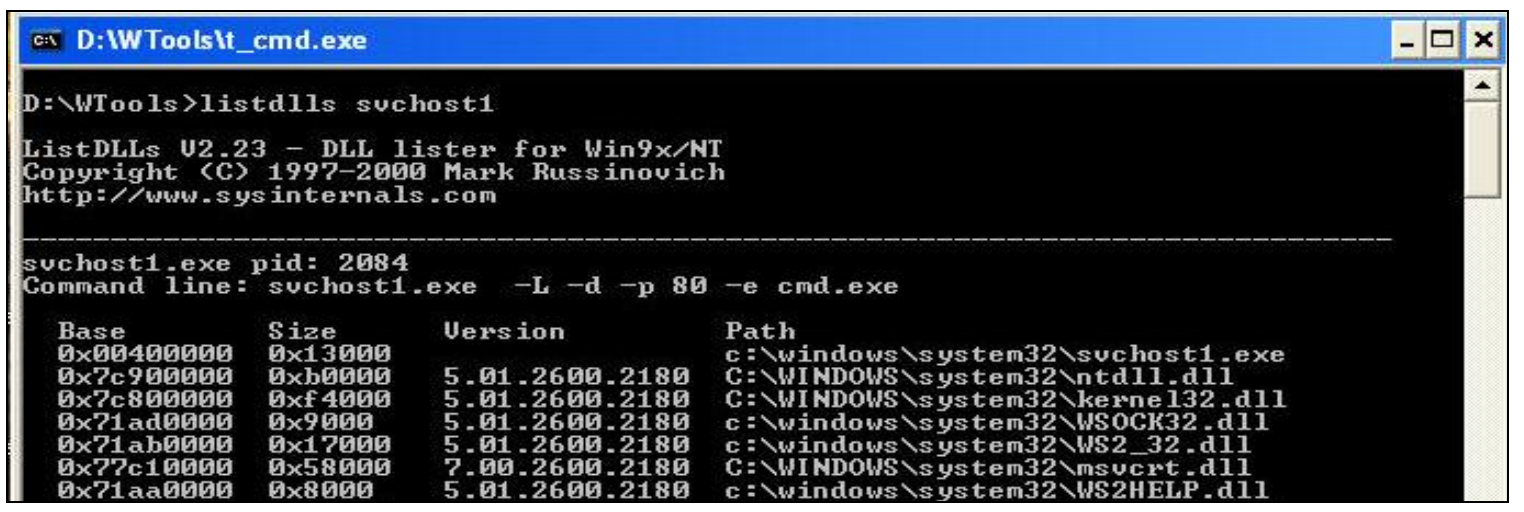




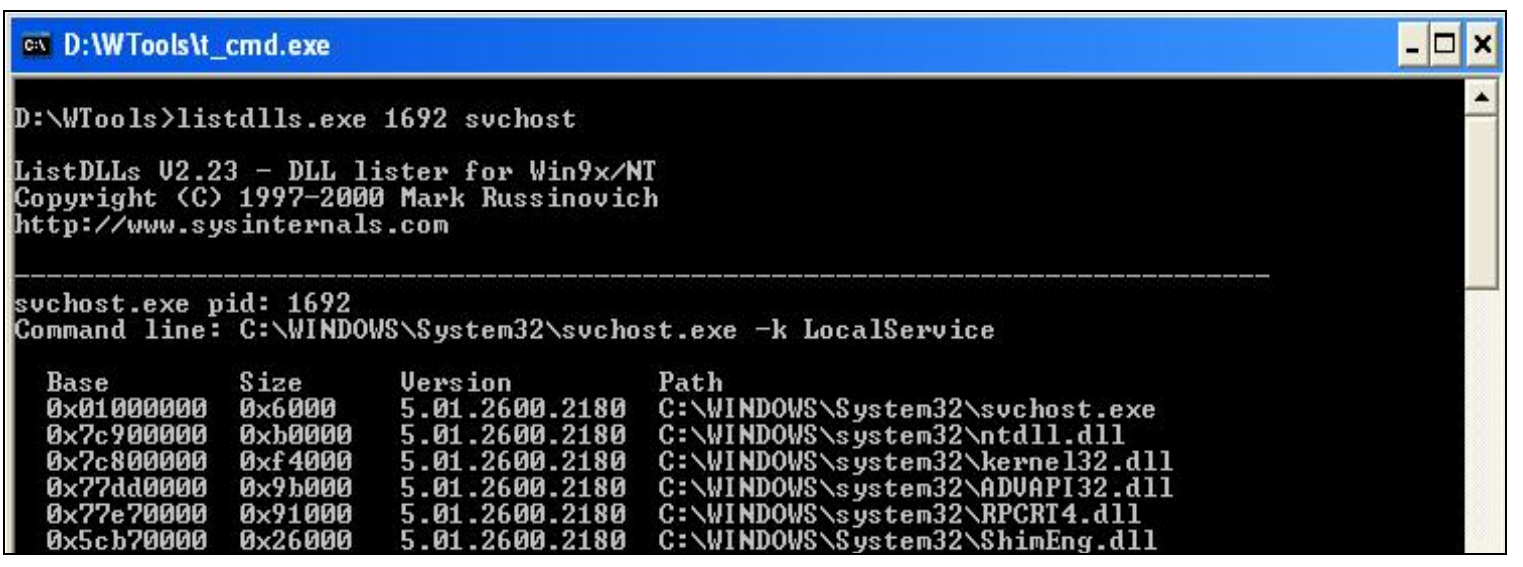

Figure 58: Using ListDLLs to Examine a Suspected Rogue Process

ListDLLs can also be used to display the command lines associated with suspected and legitimate processes. Differences could indicate a rogue process.

\section{Ldd (Linux)}

The native ldd command displays the shared object files to which an executing binary links. In Linux, each running process or application has loaded or shared libraries.

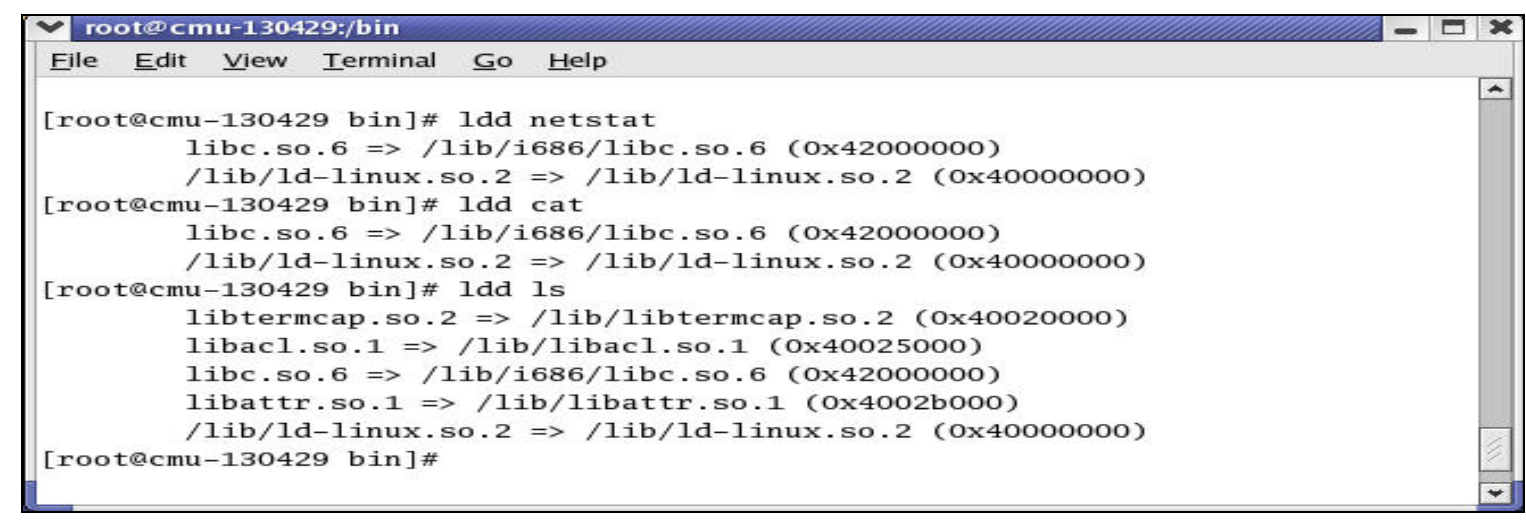

\section{Figure 59: The Idd Command}

In the following output, ldd was used to compare the shared libraries of a known Apache server binary ( $h t t p d$ ) to a so-called Apache server binary (apache). The list of shared library files for the apache binary, however, is significantly smaller than the list for httpd, which makes apache suspect.

\$ ldd ./apache (Unknown)

libc.so.6 => /lib/libc.so.6 (0x4001f000)

/lib/ld-linux.so.2 => /lib/ld-linux.so.2 (0x40000000) 


\section{\$ ldd /usr/sbin/httpd (Known)}

$$
\begin{aligned}
& \text { libm.so.6 => /lib/libm.so.6 (0x7002c000) } \\
& \text { libcrypt.so.1 => /lib/libcrypt.so.1 (0x700c4000) } \\
& \text { libdb.so.2 => /lib/libdb.so.2 (0x70100000) } \\
& \text { libdb2.so.2 => /lib/libdb2.so.2 (0x70120000) } \\
& \text { libexpat.so.1 => /usr/lib/libexpat.so.1 (0x70180000) } \\
& \text { libdl.so.2 => /lib/libdl.so.2 (0x701b4000) } \\
& \text { libc.so.6 => /lib/libc.so.6 (0x701c8000) } \\
& \text { /lib/ld-linux.so.2 => /lib/ld-linux.so.2 (0x70000000) }
\end{aligned}
$$

\begin{tabular}{|c|c|c|c|}
\hline \multicolumn{3}{|l|}{$\checkmark$ root@localhost:/lib } & \multirow[t]{2}{*}{$-\square x$} \\
\hline File Edit View I & Help & & \\
\hline \multicolumn{3}{|c|}{ [root@localhost lib]\# ls } & $A$ \\
\hline cpp & libiw.so. 25 & libnss_nis.so.2 & \\
\hline i686 & liblvm-10.so & libnss_winbind.so & \\
\hline iptables & liblvm-10.so.1 & libnss_winbind.so.2 & \\
\hline kbd & liblvm-10.so.1.0 & libnss_wins.so & \\
\hline $1 d-2.2 .93$. so & libm-2.2.93.so & libnss_wins.so.2 & \\
\hline 1d-linux.so. 2 & libm.so. 6 & libpamc. so. 0 & \\
\hline libacl.so.1 & libNoVersion-2.2.93.so & libpamc.so.0.75 & \\
\hline libacl.so.1.0.0 & libNoVersion.so.1 & libpam_misc.so. 0 & \\
\hline libanl-2.2.93.so & libnsl-2.2.93.so & libpam_misc.so.0.75 & \\
\hline libanl.so.1 & libnsl.so.1 & libpam.so.0 & \\
\hline
\end{tabular}

\section{Ls (Linux)}

The native ls command can be used to display the shared libraries to which each executing binary links. In Figure 60, ls is used to display contents of the lib folder.

Figure 60: The Is Command

For more details about default library locations, see Wheeler's discussion [Wheeler 03]. 


\section{Volatile Network Information}

Open connections

Open ports and sockets

Routing information and configuration

Network interface status and configuration

ARP cache

๑) 2005 Carnegie Mellon University

Module 3: Collecting Volatile Data

\subsubsection{Volatile Network Information}

The types of volatile network information included in this section are not exhaustive, but they are key indictors of the network state that can help you unravel the security incident.

If the machine is standalone or offline, skip this step. 


\section{Open Connections and Ports}

\section{Purpose:}

Identify all live connections

Identify all open ports

Insight into whom or what the system has been transmitting to or communicating with

\section{What to look for:}

Unfamiliar established connections

Unfamiliar IP addresses

Unfamiliar ports open

Network card configuration

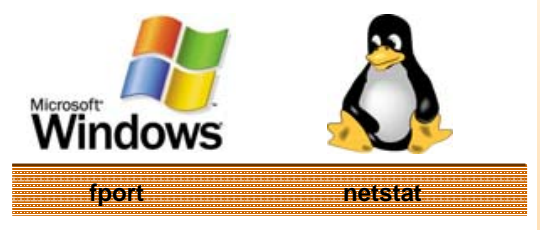

psservice

ifconfig

netstat

(c) 2005 Carnegie Mellon University

Module 3: Collecting Volatile Data

\subsubsection{Open Connections and Ports}

To begin collecting information about open connections and ports, examine the suspicious computer to see if a any type of cable connects it to a network. After you establish all live connections, collect and document the associated IP addresses and open ports. This initial collection of network information may be enough for you to determine whether the security incident was caused remotely or locally.

As you use the tools in this section, look for the following:

- unfamiliar IP addresses and ports

- legitimate (established through commands like telnet, ssh, ftp, and netuse) or backdoor connections on abnormal ports

- a promiscuously configured network card interface

Do not expect to know what each open port on the suspicious computer is used for. To look up a port, visit www.treachery.net/tools/ports/lookup.cgi. 


\section{Fport (Windows)}

The Fport freeware utility from Foundstone, like the native netstat -an command, displays all open TCP/IP and UDP ports and maps them to the owning application. Fport also maps those ports to running processes with the PID, process name, and path.

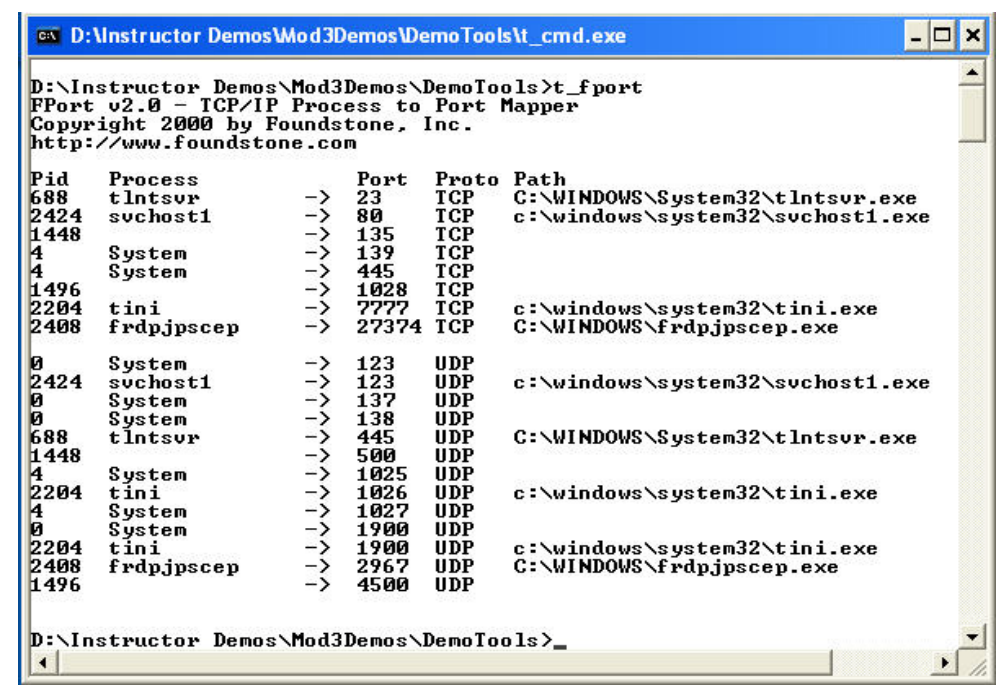

Figure 61: Fport

A GUI-based commercial version of Fport is available at http://www.foundstone.com.

\section{PsService (Windows)}

Sysinternals' PsService freeware utility acts as

a service viewer and controller for Windows NT/2K2000. Like the SC utility that's included in the Windows NT and Windows 2000 Resource Kits, PsService displays the status, configuration, and dependencies of a service, and allows you to start, stop, pause, resume, and restart them. Unlike the SC utility, PsService enables you to log on to a remote system using a different account, for cases when the account from which you run it doesn't have required permissions on the remote system. PsService includes a unique service-search capability, which identifies active instances of a service on your network. You would use the search feature if you wanted to locate systems running DHCP servers, for instance [Russinovich 03]. 


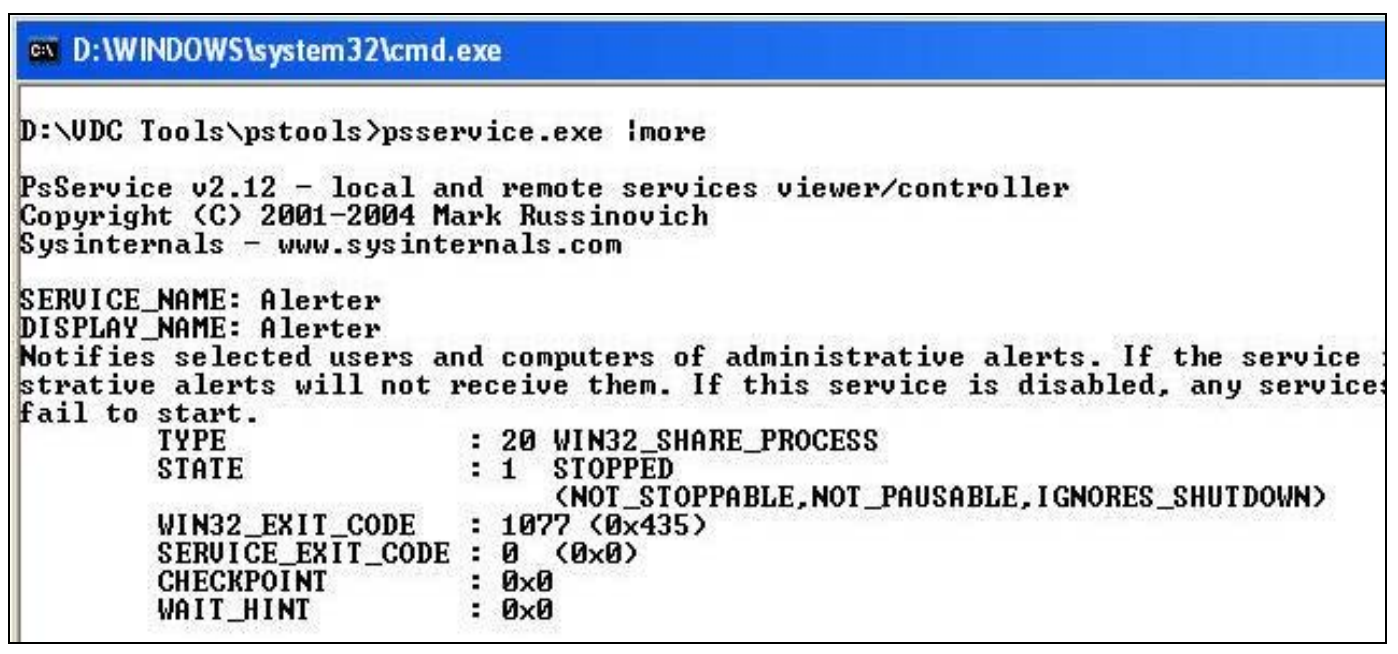

Figure 62: PsService

\section{PromiscDetect (Windows)}

NTSecurity.nu's freeware utility PromiscDetect checks to see if the network adaptor(s) are running in promiscuous mode, which may indicate that a sniffer is running on the suspicious computer. To determine the adapter's mode, look at the PromiscDetect's output. If the filters returned are set at Directed, Multicast, and Broadcast, the network card interface is not in promiscuous mode.

PromiscDetect is available at http://ntsecurity.nu/toolbox/promiscdetect.

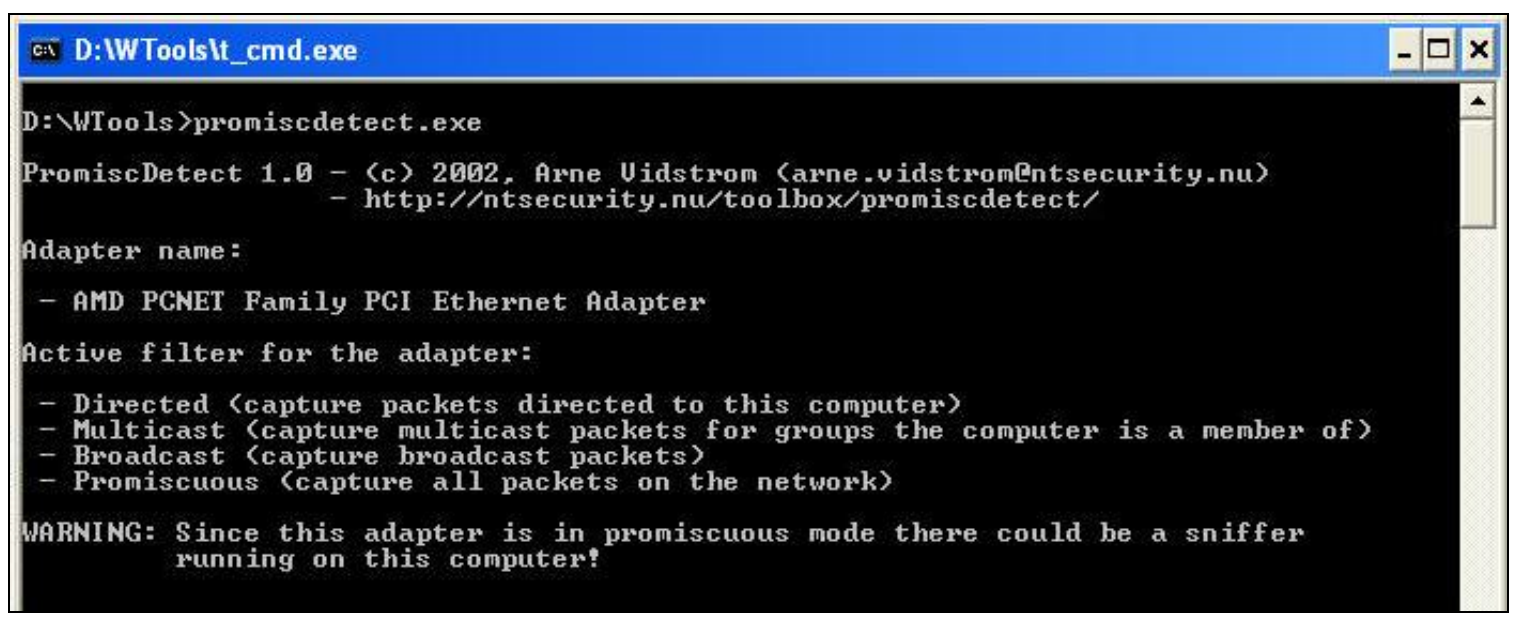

Figure 63: PromiscDetect

\section{Netstat - anb (Windows)}

The native netstat - anb command displays a list of current TCP/IP connections. It also displays the protocol of the connection, the local address (MAC address), the IP address, and 
the connection state. For Windows XP systems, netstat has an additional switch (-0) that displays the PID for the process associated with each connection.

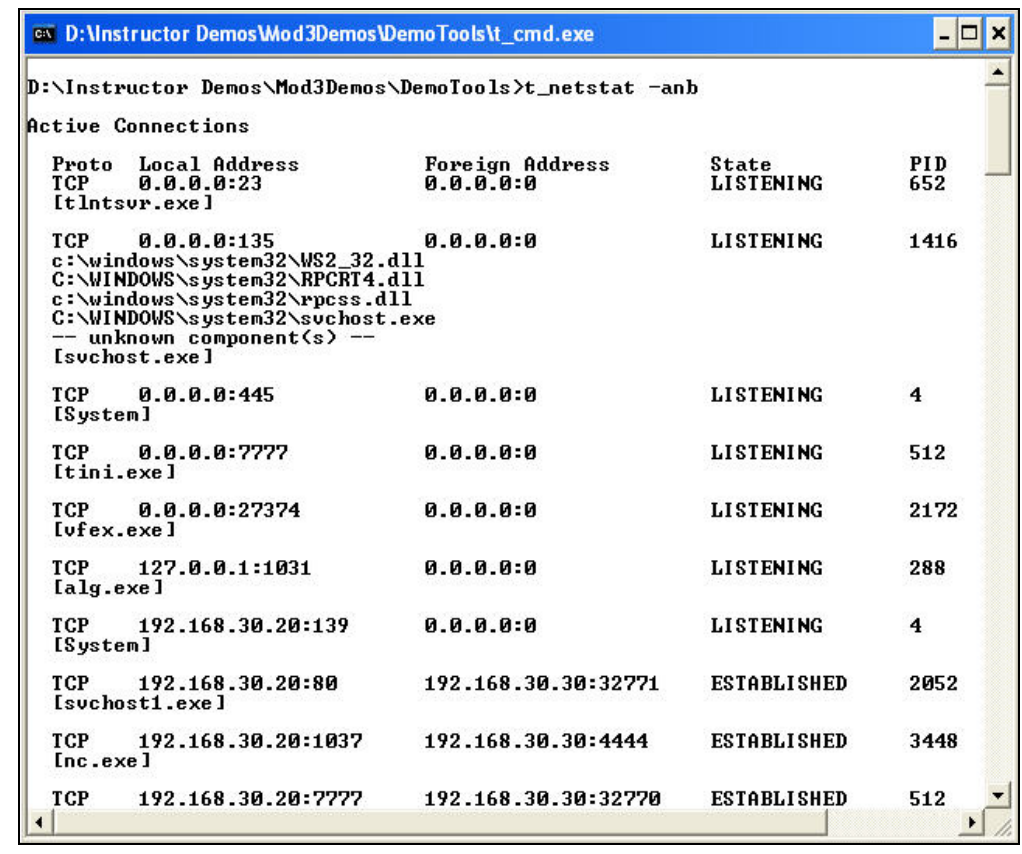

Figure 64: The netstat -anb Command

\section{Nbstat (Windows)}

The native nbtstat command can collect information about recent network connections using the NBT (NetBIOS over TCP/IP) protocol. The nbtstat $-s$ command displays the session table for the local system with destination IP addresses, which helps you identify mapped connections to shared drives on other computers.

\section{Net (Windows)}

The native net command can be used to determine if the local machine has any network shares or is connected to any network shares. It also lists files that have been opened on the local system via a session established with a remote system over NetBios. 


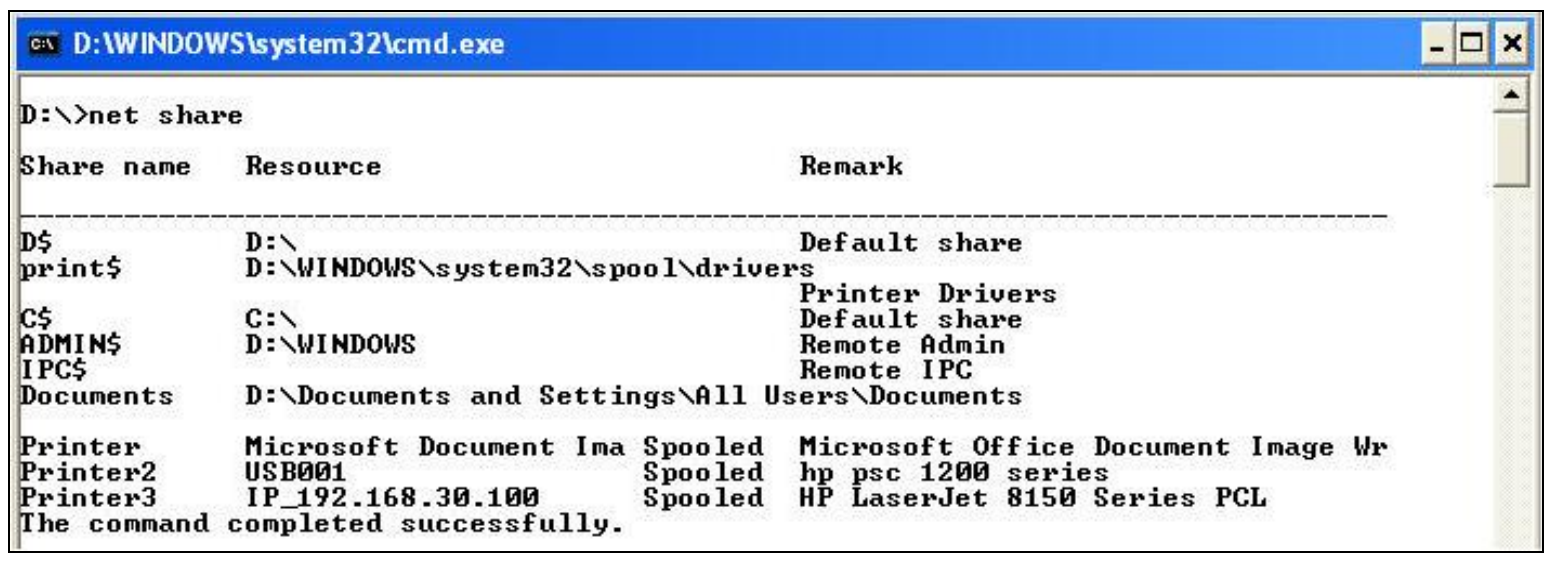

Figure 65: The net Command

\section{Netstat (Linux)}

The native netstat command displays information on active sockets, routing tables, interfaces, masquerade connections, and multicast memberships so that you can map processes to open connections and sockets. Without parameters, netstat lists open sockets. With the $-\mathrm{p}$ parameter, it shows the process ID and name of the program that owns the socket. The netstat -anp command displays the protocol, local address, foreign address, state, and program responsible for the open connection.

\begin{tabular}{|c|c|c|c|c|c|c|c|c|}
\hline \multicolumn{9}{|c|}{$\sqrt{v \text { rootelocalhost: bin }}$} \\
\hline \multicolumn{9}{|c|}{ Ele Edit Vew Ieminal $\mathrm{G}_{0}$ Help } \\
\hline \multirow{2}{*}{\multicolumn{9}{|c|}{$\begin{array}{l}\text { [rootelocalhost bin]" netstat -anp } \\
\text { Active Internet connections (servers and established) }\end{array}$}} \\
\hline & & & & & & & & \\
\hline \multicolumn{4}{|c|}{ Proto Recv- $Q$ Send-Q Local Address } & \multicolumn{3}{|c|}{ Foreign Address } & State & PID/Progran nane \\
\hline tcp & 0 & \multicolumn{2}{|c|}{$00.0 .0 .0: 32768$} & \multicolumn{3}{|c|}{$0.0 .0 .0:^{*}$} & LISTEN & $612 /$ rpc.statd \\
\hline tep & 0 & \multicolumn{2}{|c|}{$0127 \cdot 0.0 .1: 32769$} & \multicolumn{3}{|c|}{$0.0 .0 .0: *$} & LISTEN & $745 / x$ inetd \\
\hline tep & 0 & \multicolumn{2}{|l|}{$00.0 .0 .0: 111$} & \multicolumn{3}{|c|}{$0.0 .0 .0:^{*}$} & LISTEN & $593 /$ portnap \\
\hline tcp & 0 & \multicolumn{2}{|l|}{$00.0 .0 .0: 6000$} & \multicolumn{3}{|c|}{$0.0 .0 .0:^{*}$} & LISTEN & $922 / X$ \\
\hline tcp & 0 & \multicolumn{2}{|c|}{$00.0 .0 .0: 22$} & \multicolumn{3}{|c|}{$0.0 .0 .0: *$} & LISTEN & $731 /$ sshd \\
\hline $\operatorname{tcp}$ & 0 & \multicolumn{2}{|c|}{$0127 \cdot 0.0 .1: 25$} & \multicolumn{3}{|c|}{$0.0 .0 .0 *^{*}$} & LISIEN & 768/sendnail: accep \\
\hline tcp & 0 & \multicolumn{2}{|c|}{$0128.237 .230 .253: 32779$} & \multicolumn{3}{|c|}{$216.239 .39 .99: 80$} & \multicolumn{2}{|c|}{ ESTABLISHED 3559/nozilla-bin } \\
\hline tcp & 0 & \multicolumn{2}{|c|}{$0128.237 .230 .253: 32774$} & \multicolumn{3}{|c|}{$207.126 .111 .202: 80$} & TIME_WAIT & - \\
\hline tcp & 0 & \multicolumn{2}{|c|}{$0128.237 .230 .253: 32775$} & \multicolumn{3}{|c|}{$207.126 .111 .202: 80$} & TINE_WAIT & - \\
\hline udp & 0 & \multicolumn{2}{|c|}{$00.0 .0 .0: 32768$} & \multicolumn{3}{|c|}{$0.0 .0 .0:^{*}$} & & $612 /$ rpc.statd \\
\hline udp & 0 & \multicolumn{2}{|l|}{$00.0 .0 .0: 68$} & \multicolumn{3}{|c|}{$0.0 .0 .00^{*}$} & & 525/dhclient \\
\hline udp & 0 & \multicolumn{2}{|l|}{$00.0 .0 .0: 111$} & \multicolumn{3}{|c|}{$0.0 .0 .0:^{*}$} & & 593/portnap \\
\hline \multicolumn{9}{|c|}{ Active UNIX donain sockets (servers and established) } \\
\hline Proto RefCn & nt Flags & Type & State & & I-Node & PID/Pro & ram nane & Path \\
\hline unix 2 & [ $\mathrm{ACC}$ ] & STREAM & LISTEN & & 1610 & $788 / \mathrm{gpm}$ & & /dev/gpnctl \\
\hline unix 11 & [] & DGRAM & & & 1027 & $572 /$ sys & & /dev/log \\
\hline unix 2 & [ $\mathrm{ACC}$ ] & STREAM & LISTEN & & 1654 & $826 / x f s$ & & $/ \operatorname{tmp} /$.font-unix/fs 7100 \\
\hline unix 2 & [ $\mathrm{ACC}$ ] & STREAM & LISTEN & & 1762 & $922 / \mathrm{X}$ & & $/ \operatorname{tmp} / . \times 11-$ unix $/ X_{0}$ \\
\hline unix 2 & [ $\mathrm{ACC}$ ] & STREAM & LISTEN & & 1780 & 939/ssh & agent & /trp/ssh-XXErs55n/agent. 925 \\
\hline unix 2 & {$[\mathrm{ACC}]$} & STREAM & LISTEN & & 1902 & 925/gno & -session $/ t$ & /tmp/.ICE-unix/925 \\
\hline unix 2 & [ $\mathrm{ACC}$ ] & STREAM & LISTEN & & 1813 & $951 / 8 \mathrm{co}$ & & /tmp/orbit-root/linc-3b7-0- \\
\hline 7dia40ef330 & & & & & & & & \\
\hline unix 2 & [ $\mathrm{ACC}$ ] & SIREAM & LISTEN & & 45197 & 2176/oa & & /tmp/orbit-root/orb-9672428 \\
\hline
\end{tabular}

Figure 66: The netstat -anp Command 


\section{/var/log/messages (Linux)}

Look at $/ \mathrm{var} / \mathrm{log} / \mathrm{messages}$ to determine if the network interface card is in promiscuous mode.

\begin{tabular}{|l|}
\hline File Edit View Ierminal Go Help \\
[root@localhost /]\# tail /var/log/messages \\
Nov 18 12:27:14 localhost modprobe: modprobe: Can't locate module sound-service \\
$-0-3$ \\
Nov 18 12:27:17 localhost kernel: Attached scsi CD-R0M sr0 at scsil, channel 0, \\
id 0,1 lun 0 \\
Nov 18 12:27:17 localhost kernel: sro: scsi3-mmc drive: 1x/1x xa/form2 cdda tra \\
Y \\
Nov 18 12:27:17 localhost kernel: Uniform CD-RoM driver Revision: 3.12 \\
Nov 18 14:56:00 localhost kernel: eth0: Promiscuous mode enabled. \\
Nov 18 14:56:00 localhost kernel: device eth0 entered promiscuous mode \\
Nov 18 14:57:21 localhost kernel: device eth0 left promiscuous mode \\
Nov 18 15:02:13 localhost kernel: eth0: Promiscuous mode enabled. \\
Nov 18 15:02:13 localhost kernel: device eth0 entered promiscuous mode \\
Nov 18 15:05:19 localhost kernel: device eth0 left promiscuous mode \\
[root@localhost /]\#
\end{tabular}

\section{Figure 67: /var/log/messages}

\section{Ifconfig (Linux)}

The native ifconfig command displays the current configuration for a network interface.

Displayed information includes IP address, gateway, DNS servers, and promiscuous mode detection. In Figure 68, there would be a Promisc flag next to Multicast to alert you that there is a network sniffer on this machine.

\begin{tabular}{|c|c|}
\hline \multicolumn{2}{|c|}{$\mathbf{V}$ rootelocalhost:/bin } \\
\hline File & View Ierminal Go Help \\
\hline \multicolumn{2}{|c|}{ [root@localhost bin]\# ifconfig -a } \\
\hline etho & Link encap:Ethernet HWaddr $00: 50: 56: 00: \mathrm{FF}: 97$ \\
\hline & inet addr:128.237.230.253 Bcast:128.237.255.255 Mask:255.255.224.0 \\
\hline & UP BROADCAST RUNNING MULTICAST MTU:1500 Metric:1 \\
\hline & RX packets: 801515 errors:0 dropped:0 overruns:0 frame:0 \\
\hline & IX packets:523 errors:0 dropped:0 overruns:0 carrier:0 \\
\hline & collisions: 0 txqueuelen: 100 \\
\hline & RX bytes: $98894160(94.3 \mathrm{Mb})$ TX bytes:60403 $(58.9 \mathrm{~kb})$ \\
\hline & Interrupt:10 Base address:0x1080 \\
\hline
\end{tabular}

Figure 68: The ifconfig Command 


\section{Routing Information}

Why:

To determine the recent connections made to the machine and the configuration of the routing table

What to look for:

Statically added routes

Unfamiliar IP addresses and MAC addresses

(c) 2005 Carnegie Mellon University

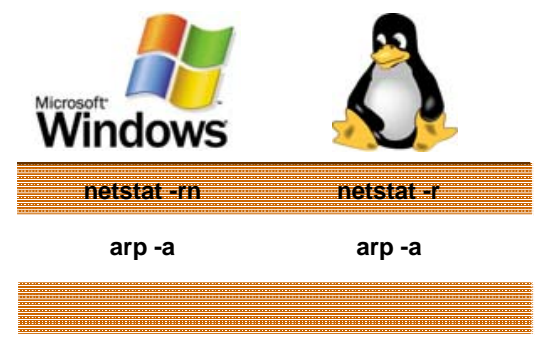

Module 3: Collecting Volatile Data

\subsubsection{Routing Information}

As you use the tools in this section to collect routing information, be sure to pay attention to the suspicious computer's routing table. Make note of added or unfamiliar routes. Also, look at the ARP cache to identify recent connections. 


\section{Netstat, Route (Windows)}

The native netstat and route commands allow you to collect routing information. As shown in Figure 69, netstat $-r$ displays all volatile active routes for the suspicious computer.

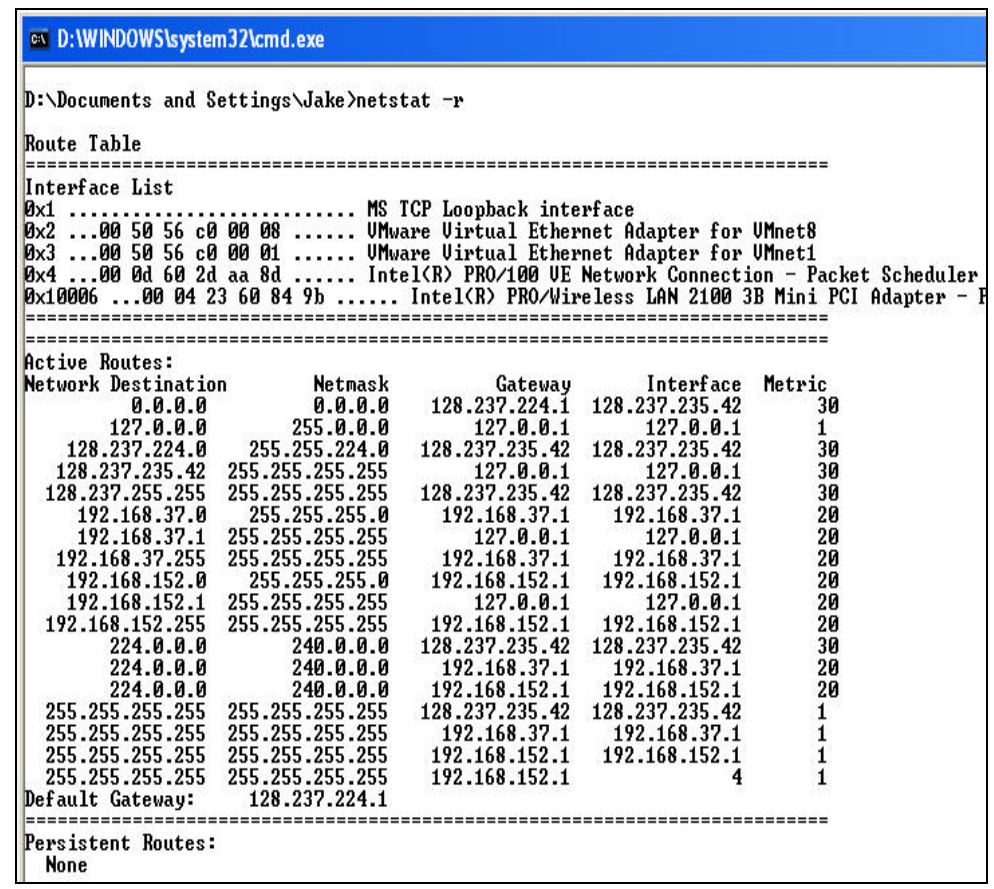

Figure 69: The Windows netstat -r Command

\section{$\operatorname{Arp}$ (Windows)}

The native arp command displays the following information about the suspicious computer's network interface: IP address, MAC address, and type (dynamic or static). The ARP cache also stores the MAC address to IP address translations for the last two minutes [Pierce 03]. The -a switch pulls the active ARP cache entries.

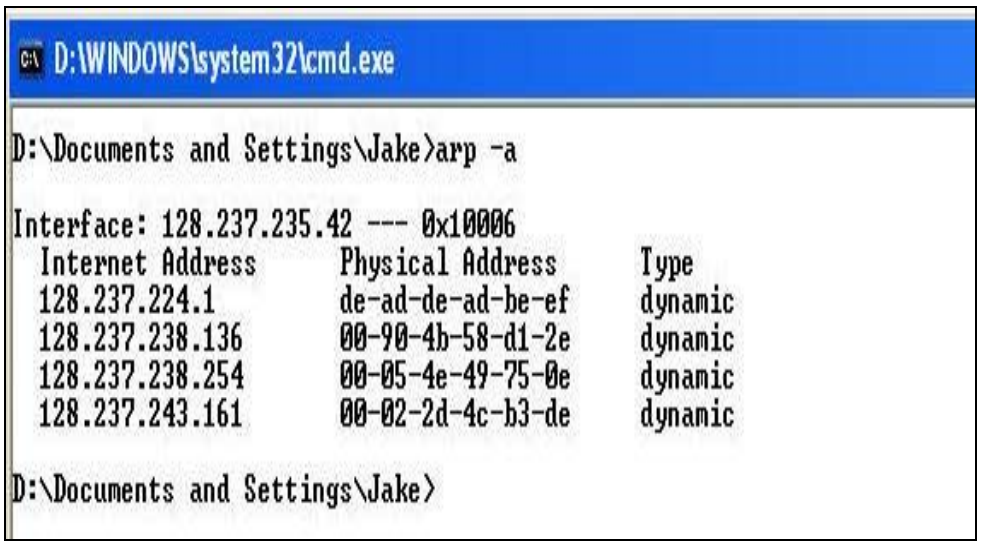

Figure 70: The Windows arp Command 


\section{Netstat (Linux)}

The native netstat -rn command displays the current routing table and gateways for all routes on the suspicious computer.

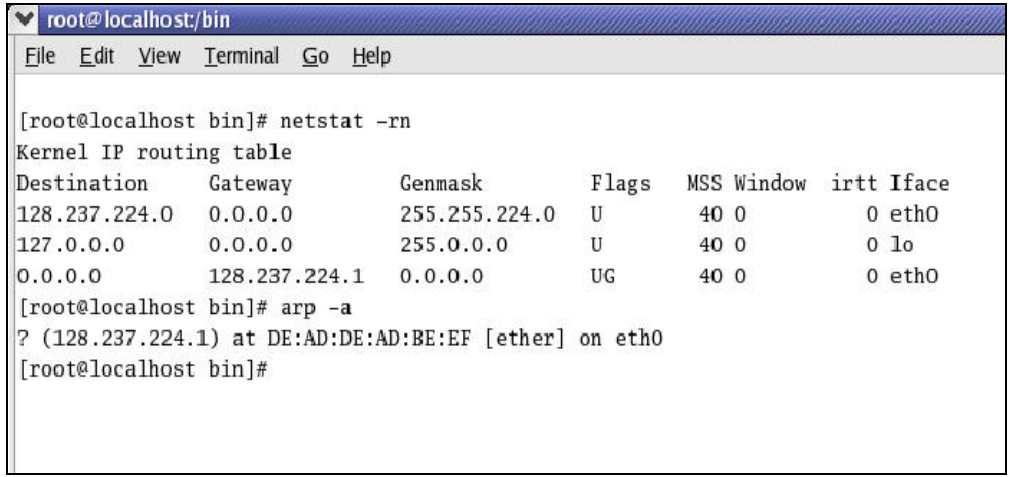

Figure 71: The Linux netstat -rn Command

\section{$\operatorname{Arp}($ Linux)}

The native arp - $a$ command displays route entries for the suspicious computer. Determine whether there are any permanent ARP cache entries or whether ARP proxies were created.

[rooteLinux_Compromised root]\# arp -a

? (192.168.30.20) at 00:0C:29:20:5A:EF [ether] on eth日

? (192.168.30.1) at 〈incomplete> on etho

[rooteLinux_Compromised root]\# -

Figure 72: The Linux arp -a Command 


\section{Summary}

Collected volatile data can lead the first responder to the root cause of the security incident.

Volatile data can be easily changed and lost.

Document all findings and actions performed during the volatile data collection process.

Use a first responder toolkit to collect volatile data.

\subsection{Summary}

The slide above summarizes the main points for Module 3 . 


\section{Review}

1. What Windows utility will allow you to collect a list of currently loaded DLLs?

2. What Linux command will allow you to collect a list of services that will start upon boot up?

3. Why is it important to collect volatile data from a live machine?

4. Why should you never use Windows or Linux native commands from the live host?

5. What utility will allow you to transmit volatile data to a remote workstation?

Module 3: Collecting Volatile Data

\subsection{Review}

1. The Listdlls.exe utility will allow you to collect a list of currently loaded DLLs.

2. The chkconfig will allow you to collect a list of services that will start upon boot up.

3. It is important to collect volatile data from a live machine for the following reasons:
a. to gain initial insight
b. to determine a logical timeline of the computer security incident
c. to determine the next course of action
d. because you have one chance to collect volatile data

4. You should never use native Windows or Linux commands on the suspect live host because the integrity of the suspect machine could be compromised.

5. The Netcat or Cryptcat utilities will allow you to transmit volatile data to a remote workstation. 


\section{Module 4: Collecting Persistent Data}

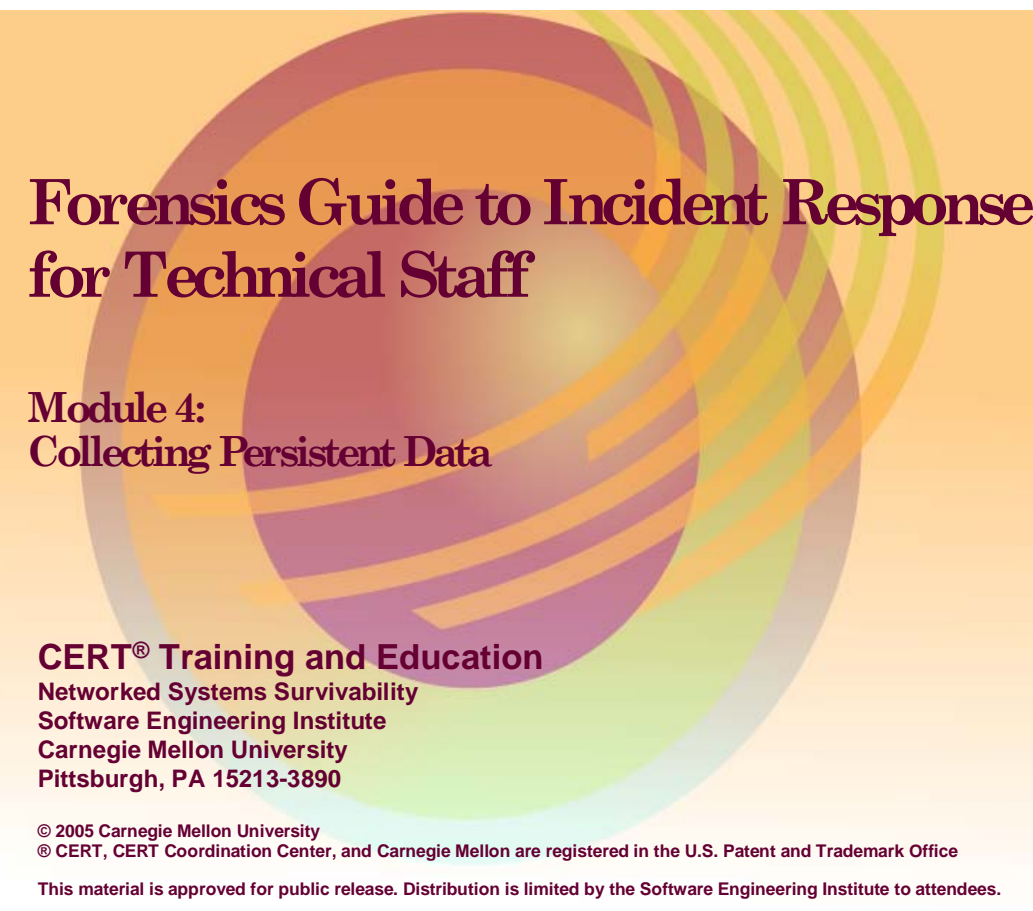

Most epistemologists concur that for effective discussion to take place, a group must first agree on what they are talking about. Therefore, we define persistent data as that which remains unchanged after the computer has been powered off. This data plays an important part in any type of computer event investigation. While not as effervescent as volatile data, persistent data can still be contaminated through improper handling.

This module reviews techniques for capturing persistent data in a forensically sound manner. It also covers the location of common persistent data types. 
Objectives

Define persistent data

Highlight importance of persistent data

Learn consequences of improper examination to persistent data

Learn proper techniques for capturing persistent data

Learn the locations of common persistent data types

\subsection{Objectives}

The primary objective of this module is to highlight the importance of handling persistent data in a forensically sound manner. It includes the following topics:

- definition of persistent data

- importance of persistent data in responding to a security event

- consequences of improper examination

- proper techniques for capturing persistent data

- locations of common persistent data types 


\section{Introduction to Persistent Data}

What is persistent data?

- Data in the physical media

- Survives machine shutdown

Why is persistent data important?

- Majority of evidence is persistent data

What problems exist in investigating persistent data?

- Contamination through improper handling

() 2005 Carnegie Mellon University

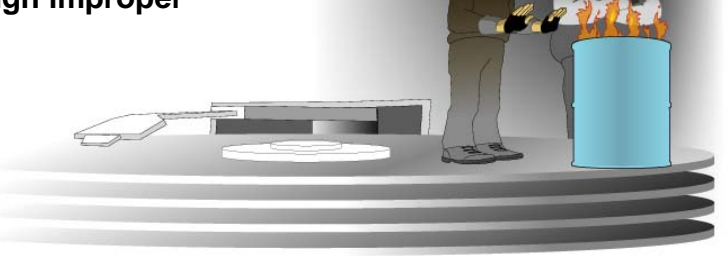

\subsection{Introduction to Persistent Data}

\subsubsection{What Is Persistent Data?}

Fundamentally, persistent data is retained and remains unchanged after the computer has been powered off. It is found on removable storage media as well as hard drives. Despite its stable nature, precautions still need to be taken to ensure proper collection.

This definition is broad enough to include data held on disks, flash memory, cell phones, and PDAs. While the details may differ, the underlying philosophy of handling persistent data is the same across the different media.

\subsubsection{Why is Persistent Data Important?}

Most forensics evidence is found in persistent data. Examples include documents, emails, Web activity, and deleted files.

\subsubsection{What Problems Exist in Investigating Persistent Data?}

Investigating persistent data is not usually complicated. With access to a computer, most users can search for files, investigate past Internet activity, or even check for the presence of inappropriate programs. However, exploring the suspicious computer in this manner will alter evidence and make it far less useful. 
Applying the needle/haystack metaphor - the best way to get the needle, if it is there, is to collect the entire haystack—highlights two issues:

1. It can take a long time to collect a haystack. Depending on the situation, it may be better to collect only those areas of the hard drive that have the highest probability of containing the information you seek. This is determined by the indicators prompting the investigation (i.e., inappropriate Web use, harassing emails, or malicious software).

2. If the collection process takes a long time, it can take even longer to comb through the haystack for the needle. 


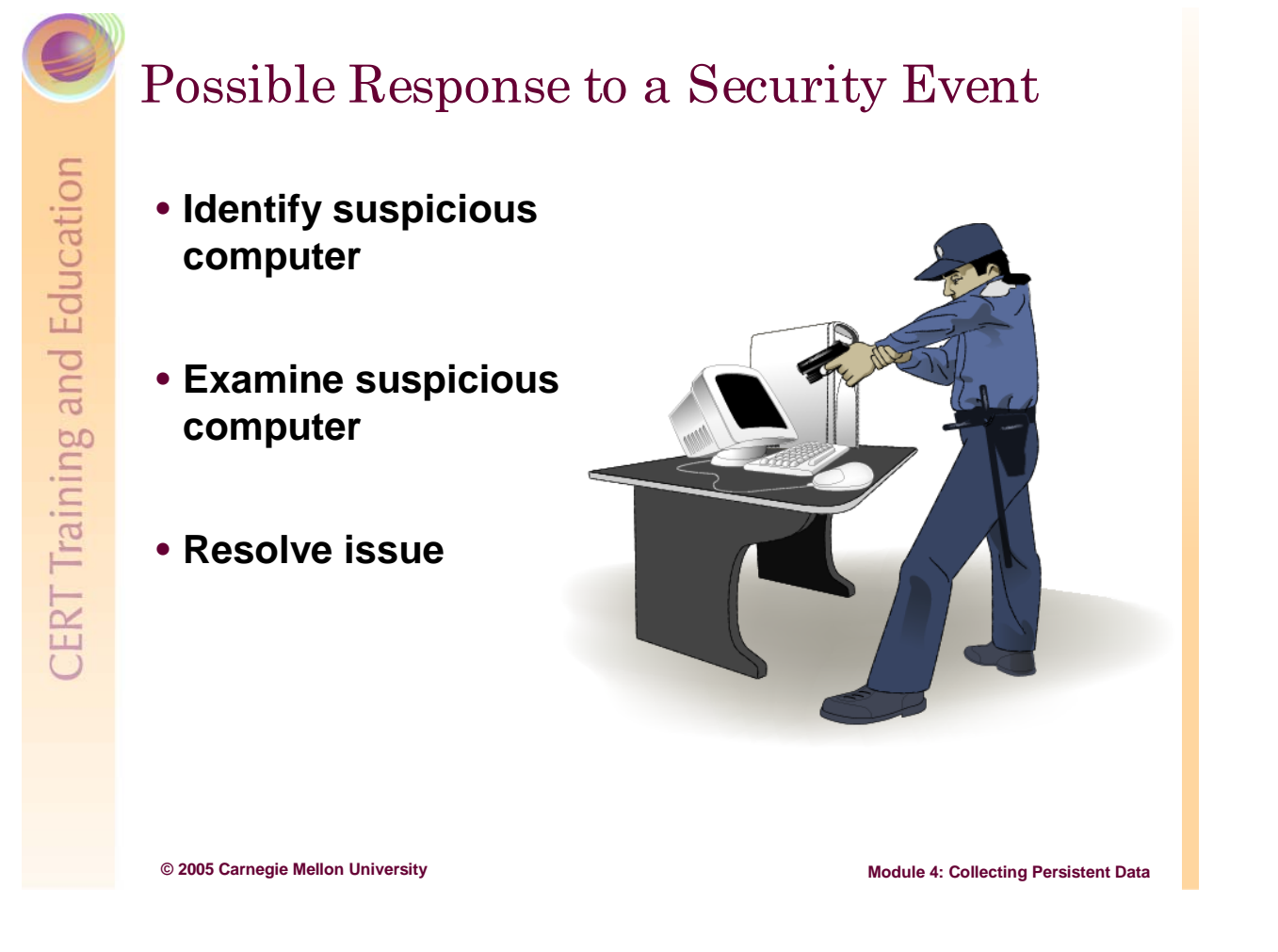

\subsection{Responding to a Security Event}

The following steps are possible responses to a security event. Unfortunately, they may not be the best response.

- Identify the suspicious computer.

- IDS alert

- user complaint of poor/odd computer performance

- user suspected of inappropriate computer use

- Examine the suspicious computer.

- examine Web history

- check or open connections

- explore file structure for suspicious files or programs

- Resolve the issue.

- patch (or re-image) machine

- confront employee

- call authorities

Once a computer comes to the attention of a system administrator, there are several possible responses to a security event, and some are better than others. It is possible to discover useful information by directly accessing the suspicious computer. By looking in the right places, an inquisitive user can, with a fair degree of accuracy, determine the user's Internet activity, examine emails, check for inappropriate material, and dig up (or at least discover the existence of) deleted files.

However, there maybe legal consequences to satisfying one’s curiosity in this manner. 


\title{
Consequences of Responses
}

\author{
Examine suspicious \\ computer
}

- Check or open connections

- Examine Web history

- Explore file structure for suspicious files or programs

\section{Resolve issue}

- Patch (or re-image) machine

- Confront employee

- Call authorities

() 2005 Carnegie Mellon University

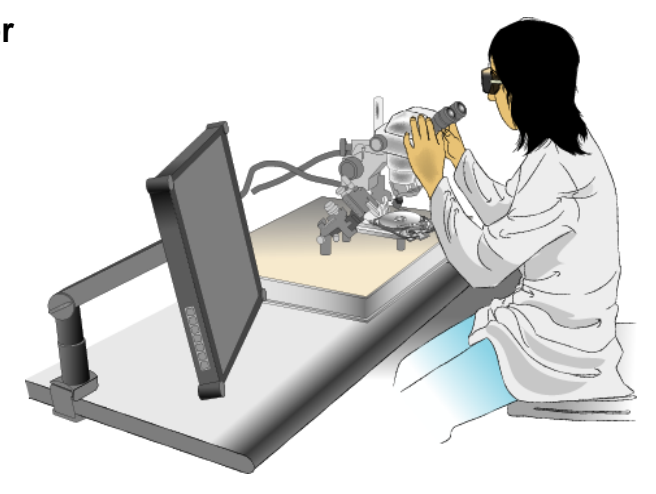

Module 4: Collecting Persistent Data

\subsubsection{Consequences of Responses}

An examination of the suspicious computer, while satisfying curiosity, will wreak havoc on the future value of the information gathered. The computer should be thought of as a crime scene. Consider this scenario: an apartment has been burglarized. The tenant calls the landlord, who comes in, opens drawers and closets, and looks behind pictures and under furniture. He finds a wallet, goes through its contents, and discovers that the owner is a tenant two floors up.

From one perspective, the landlord has just solved the case with a minimum of fuss. He knows the identity of the burglar and can confront the perpetrator and attempt to resolve the situation. Once the perpetrator is confronted, his conscience may be pricked so that he is motivated to return any stolen property and promises not to break into any more apartments. After all, he was only testing the apartment's integrity. He wasn't really stealing anything; just taking temporary custody of certain objects to highlight potential security concerns.

From another perspective, the landlord's enthusiasm to solve the problem really complicated the situation. By contaminating the crime scene and handling the evidence, the landlord has made it difficult for forensic examiners to develop a case against the thief that could be successfully prosecuted. By confronting the thief, the landlord has warned him that his identity and illicit activities have been discovered. The thief may now take additional measures during his next burglary to remain undetected. 
While the analogy is not perfect, it does illustrate some of the problems facing system administrators when responding to a security event. There will be times when it is clear that a forensic examiner needs to be called in. There will also be times when it is clear that the problem can be easily fixed and forgotten. This module is for all the times in between. By jumping straight into a suspicious computer, timestamps are changed and evidence in the slack space or unallocated space may be overwritten. If data is not collected in a forensically sound manner, defense attorneys can claim that the incriminating data was planted. 


\section{Hardware Considerations}

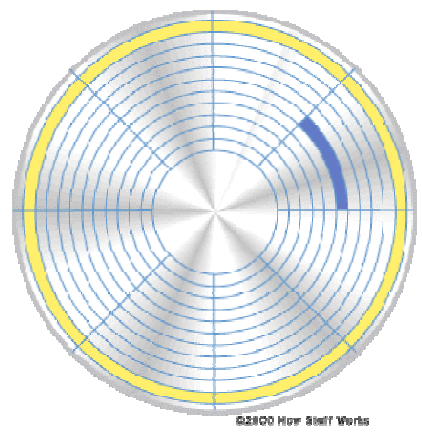

๑) 2005 Carnegie Mellon University

\section{Basic Building Blocks of Disk Storage}

- Bit: Single 1 or 0

- Byte: 8 bits

- Sector: 512 bytes (smallest addressable data unit on a disk)

- Cluster: 8 sectors (usually) assigned a status of allocated or unallocated

\subsection{Basic Building Blocks of Disk Storage}

This is a quick review of hardware information covered in the Module 2. Understanding how memory is physically organized helps in understanding how the computer stores data and how that data can linger even after it has been deleted.

Bit: A single 1 or 0

Byte: 8 bits

Sector: 512 bytes (smallest addressable data unit on a disk)

Cluster: 8 sectors (usually) assigned a status of allocated or unallocated

Figure 73 illustrates sectors and clusters on a disk. ${ }^{14}$

Figure 73: Sectors and Clusters

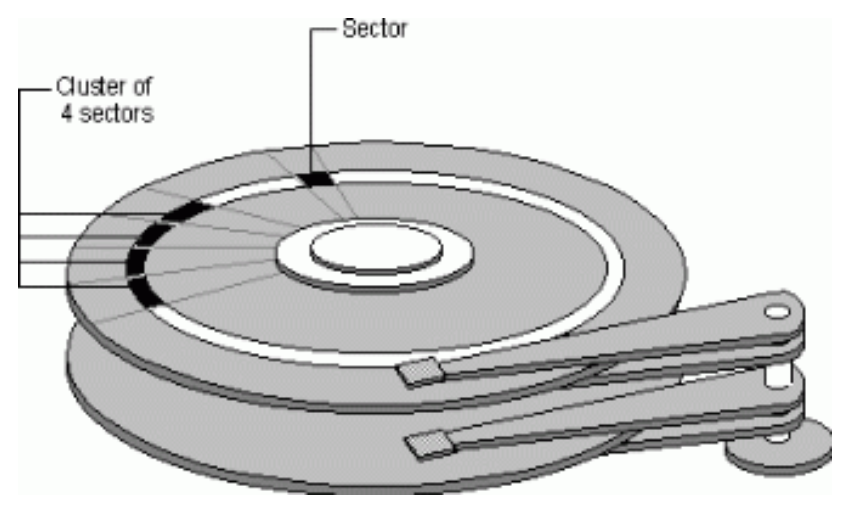

${ }^{14}$ http://www.microsoft.com/technet/archive/winntas/support/diskover.mspx 


\section{OS and Application Considerations}

\author{
Hardware, OS, and \\ applications affect how \\ you examine a system
}

(๖) 2005 Carnegie Mellon University

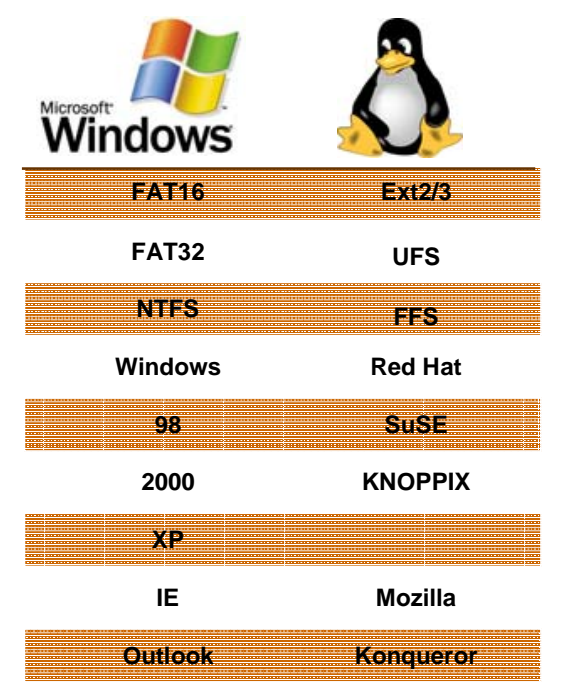

Module 4: Collecting Persistent Data

\subsection{OS and Application Considerations}

\subsubsection{Windows}

\subsubsection{FAT}

One of the earlier file systems, the file allocation table (FAT) comes in three variations:

FAT12, FAT16, and FAT32. FAT 32 is the newest variation included in Windows 95 release 2 and later [Honeynet 04]. The FAT includes an entry for each cluster on the disk except 0 and 1. Each entry includes pointers to the clusters that make up a file. The number indicates entry size (i.e., FAT32 has a 32-bit table entry).

\subsubsection{NTFS}

The new technology file system (NTFS) uses a master file table (MFT) to keep track of information on a disk. The MFT provides greater functionality and allows for larger file sizes than the FAT.

In NTFS, a file can be stored two ways. If the file is small enough, it can be stored in the MFT itself and is called a resident file. If the file is larger, it is stored in assigned clusters and called a non-resident file.

Non-resident files' names and locations are stored in the MFT. They may span several clusters, but a cluster can only be allocated to one file at a time. While clusters can be reused after a file is deleted, two different files can never share a cluster. 


\subsubsection{Linux/UNIX}

\subsubsection{Ext2/3}

Ext2/3 is the predominant file system for Linux. Ext2 partitions are organized into groups, which are made up of inodes and blocks. The inode tables contain information on files located in each group. Reiser is one flavor of the Ext2 file system. Ext3 allows for journaling in the ext file system.

Linux and UNIX developed from common ancestry and many flavors exist today. Some of the more popular ones are listed below. For more information, visit http://encyclopedia.thefreedictionary.com/UFS.

- $\quad$ UFS (UNIX File System)

A common UNIX file system

- $\quad$ FFS (Fast File System)

A common file system for FreeBSD, Open BSD, and NetBSD

- HFS and HFS+ (Hierarchical File System)

Common file systems for Macintoshes

\subsubsection{Operating Systems}

Each OS and application may organize information its own way. The Windows OS, for example, generally has Microsoft's Internet Explorer (IE) and Outlook. IE always keeps the cookie file in the same place (the IDocuments and Settings|[user] |Cookies folder). The uniformity of Windows makes it easy to find certain types of information.

Linux is more complicated; Linux flavors may organize information differently, and builds within the same flavor may even differ from each other. Because Linux systems may be highly customized, certain types of information are not always stored in the same place. For this reason, you should have a good understanding of where critical files are located for all OSs on your network before a security event occurs. 


\section{Collecting Forensic Evidence}

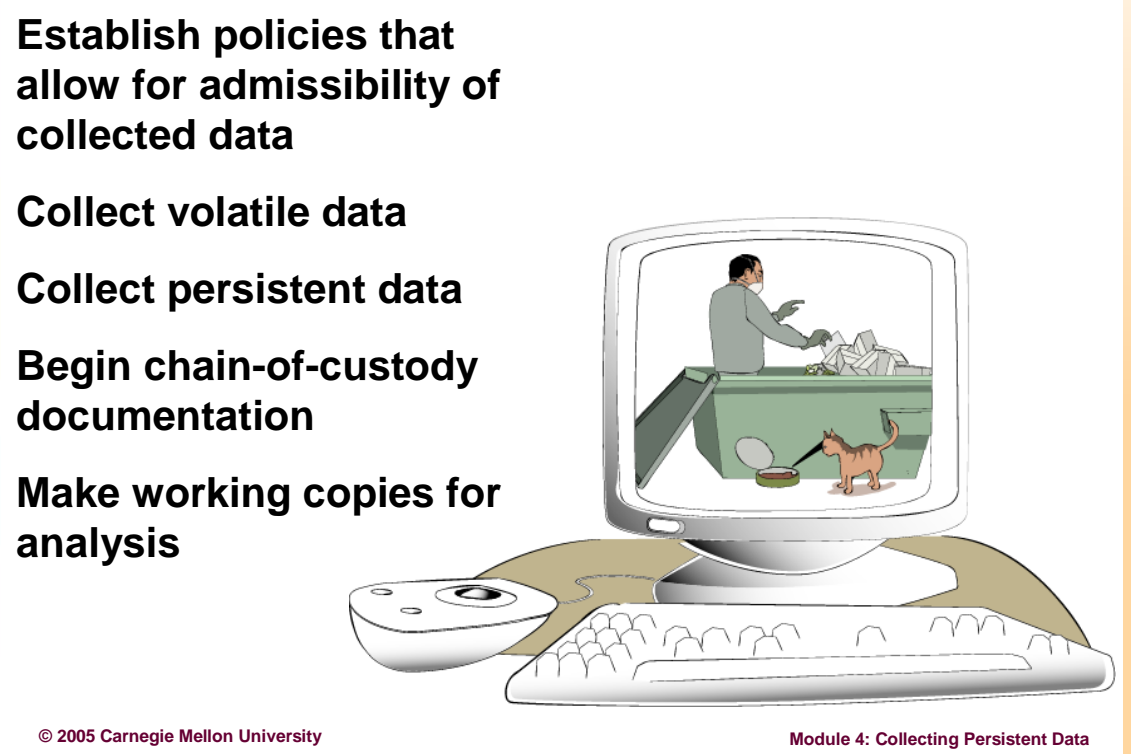

\subsection{Collecting Forensic Evidence}

Following an established procedure has benefits. As you create the procedure, potential issues can be spotted and corrected before real problems occur. As you use the procedure, steps are done in a consistent, repeatable manner. Below is a sample, high-level collection procedure:

1. Establish policies that allow for admissibility of collected data.

This is particularly important in light of admissibility of computer records. In order for a computer record to receive an exception to the heresy rules, it must be collected and be used in the normal course of business. If you plan on using any logs in your investigation and subsequent prosecution, those logs should be a part of your normal business process and not just a one-time effort.

2. Document reasons for beginning the examination.

This could be anything from poor system performance to indications that company policy has been violated. The important issue it that there is a documented reason for the investigation.

3. Document the scene.

It can be tempting to get lost in the virtual environments of networks and hard drives when responding to a security incident. One must not, however, overlook clues in the physical world. The best method to document a physical scene is to use a digital camera. Take pictures of the computer. Focus especially on the peripherals and network connections. Describe in the log what was connected to the computer as well as its 
environment. Was it in the middle of a busy cubicle farm or tucked away in a secluded corner? These details may be important to a forensic examiner.

4. Collect volatile data.

As described in Module 3, volatile data is effervescent and should be collected before nonvolatile data.

5. Collect persistent data and document each step.

a. Calculate hash values of the suspicious computer's disk/files.

b. Perform a bit-stream duplicate image/copy.

c. Calculate the hash values of the newly created master disk/file image to verify authenticity.

6. Begin chain-of-custody documentation (original evidence and master copy) and document each step.

a. Make working copies for analysis and verify their authenticity by calculating hash values.

b. Begin initial analysis to determine if additional resources are necessary. 


\section{To Shut Down or Not Shut Down...}

There are several schools of thought when presented with a suspicious computer:

- Shut down the computer

- Pull the plug

- Examine live system

Answer: IT DEPENDS

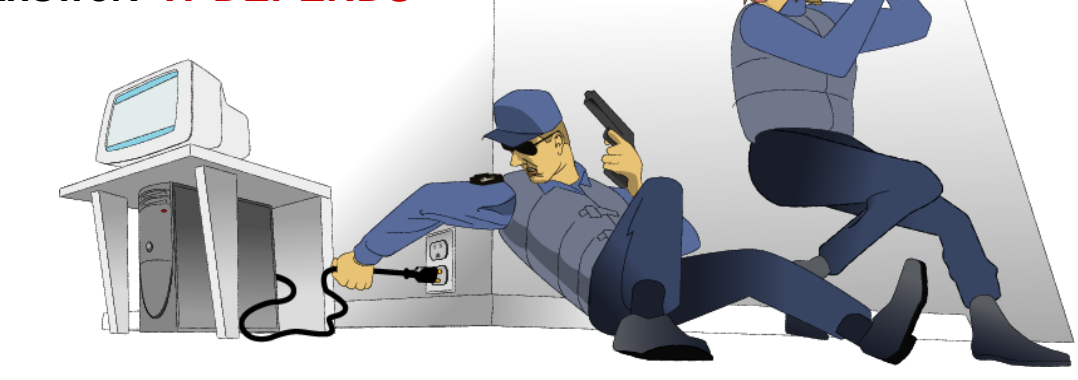

(๔) 2005 Carnegie Mellon University

Module 4: Collecting Persistent Data

\subsubsection{To Shut Down or Not to Shut Down...}

It is accepted that the action of switching off the computer may mean that a small amount of evidence may be unrecoverable if it has not been saved to the memory but the integrity of the evidence already present will be retained [ACPO 03].

Volatile data must be collected from a live system. Collecting nonvolatile data is a different story. Shutting down a system may trigger malicious software (malware) to erase evidence. If you suspect that malware is present, just pull the plug. Understanding the principles behind nonvolatile data collection will assist those responding to a security event to make the decision that best fits the circumstances of the security incident.

After a computer has been turned off, there are two options:

1. Remove the hard drive and mount it read-only on a forensic examination computer.

2. If you can not remove the hard drive, boot the computer from a safe boot disk, like Knoppix STD or Helix. Both are loaded with the necessary forensic tools to image the suspicious drive and send it across the network to another location.

Some computers may be running critical processes and cannot be shut down. In a case like this, it is vital to have a response CD as described in Module 2. 


\section{Creating a Disk Image Using dd}

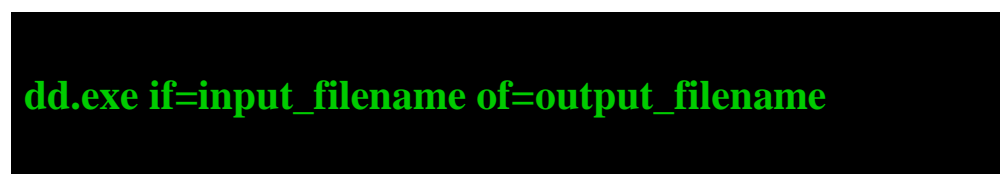

or

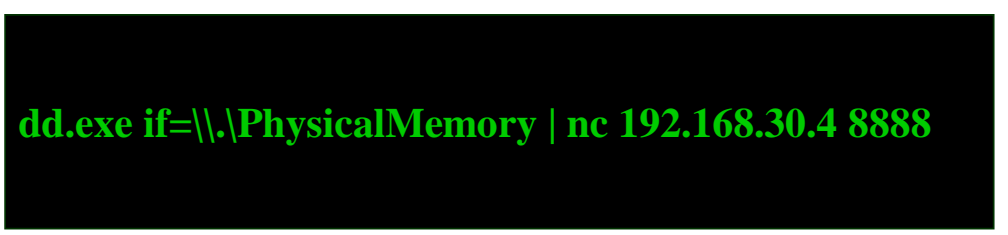

๑๑) 2005 Carnegie Mellon University

Module 4: Collecting Persistent Data

\subsubsection{Creating a Disk Image Using dd}

There are several tools that can image a disk or partition. We will use dd.exe, a small but powerful tool available for Windows and Linux. Following are some basic commands for dd [Grundy 04]. For Linux systems, most versions have dd as a native tool. For Windows systems, download dd from http://uranus.it.swin.edu.au/ jn/linux/rawwrite/dd.htm.

The basic syntax for dd.exe is explained in Figure 74.

$$
d d \text {. exe if }=/ \text { dey/hda of }=
$$

Target file to be imaged

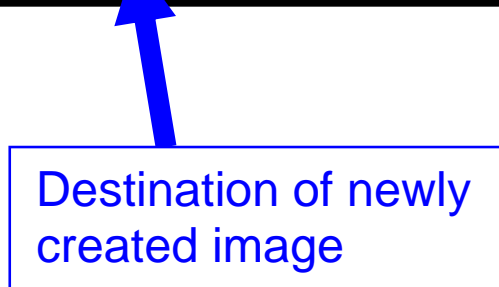

Figure 74: dd Syntax 


\section{Persistent Data Types: System Files}

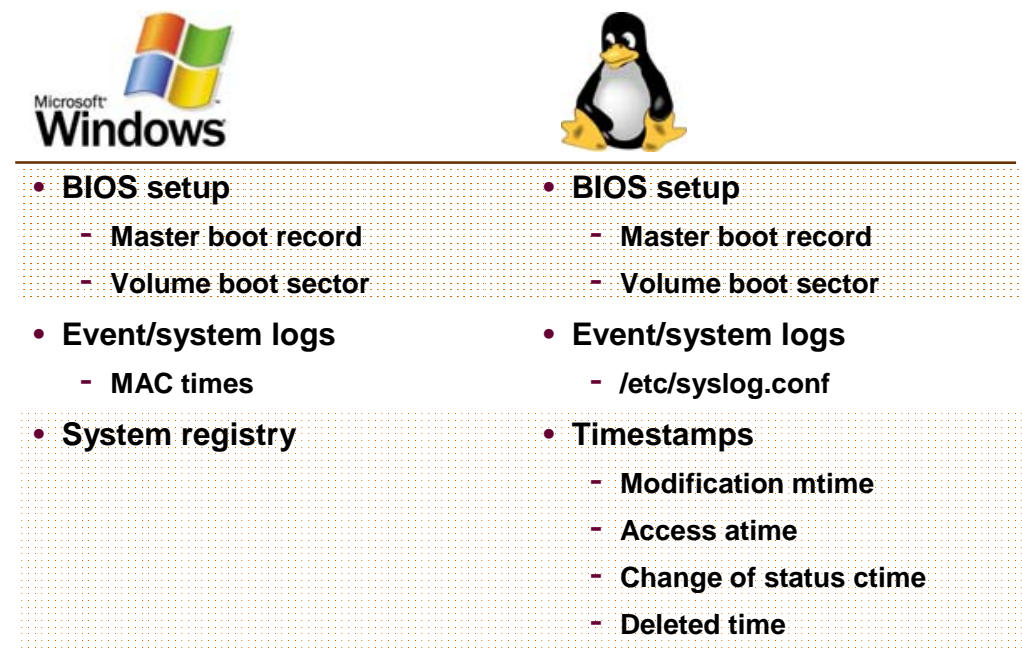

๑) 2005 Carnegie Mellon University

Module 4: Collecting Persistent Data

\subsection{Persistent Data Types}

\subsubsection{System Files}

\subsubsection{Windows}

\section{Basic Input/Output System (BIOS) Setup}

- Stored in ROM and executed when the computer boots, these commands instruct the computer on how to read the physical disks.

- The partition table and boot information are on dedicated tracks. These tracks are not visible to the file utilities that normally access the partitions or file systems.

- An analysis of a complete hard drive image with a forensic tool or hex editor is necessary.

\section{Boot Records}

A single hard drive can be organized into multiple partitions. Because each partition could have a different OS, there are two types of boot records.

\section{Master Boot Record (MBR)}

The MBR is located on the first sector of the physical disk. It contains the information the computer needs to find the partition table and determine the OS. Accessing this boot code is one of the first things the computer does. 


\section{$\underline{\text { Volume Boot Sector }}$}

Each partition has a volume boot sector. This is used to specify the file system of that partition and provide any necessary boot code needed to mount the file system.

\section{Event/System Logs}

The key to getting useful logs is to collect them before a security incident occurs. In this case, a well developed policy pays off.

\section{System Registry}

Registry data is a source for users' settings and activities. For example, HKEY_CURRENT_USER\SoftwarelMicrosoft।Internet Explorer|TypedURLS provides a list of the typed URLs.

\begin{tabular}{|c|c|c|c|c|c|c|}
\hline 64 & egistr & y Editor & & & $-\square$ & X \\
\hline & Edit & Wiew Favorites Help & & & & \\
\hline & & $\square$ Download & Name & Type & Data & 스 \\
\hline & & Explorer Bars & ab](Default) & REG_SZ & (value not set) & \\
\hline & & \{32683183-48a0- & aburl1 & REG_SZ & http://wwww.fbi.gov/ & \\
\hline & & Extensions & abjurl10 & REG_SZ & http://wwww.dea.gov/job/agent/eligibility_quiz.html & \\
\hline & & Jielp_Menu_URLs & abjurl11 & REG_SZ & http://wwww, weather.com/ & \\
\hline & & - Informationiar & $\mathrm{ab} u \mathrm{url} 12$ & REG_Sz & My Computer & \\
\hline & & SPW & abjurl13 & REG_SZ & http://wwww.lds.org/ & \\
\hline & & + International & abjurl14 & REG_SZ & http://byu,edu!' & \\
\hline & & Main & abjurl15 & REG_SZ & http://iry.byu.edul' & \\
\hline & & $+\square$ Media & abjurl16 & REG_SZ & http://Www.upromise.com/ & \\
\hline & & $+\square$ MenuExt & abjurl17 & REG_SZ & http://Wwww.msn.com/ & \\
\hline & & $+\square$ New Windows & ab]url18 & REG_SZ & http://wwww,cmu,edu & \\
\hline & & $\square$ PageSetup & ab]url19 & REG_SZ & hettp:///Wusw.cmu.edushub & \\
\hline & & $\square$ Searchurl & $\mathrm{ab} u r \mid 2$ & REG_S2 & http://wwww , foxnews, com/ & \\
\hline & & $\square$ Security & abjurl20 & REG_SZ & http://iwwww.usajobs.com/ & \\
\hline & & 9 Services & abjurl21 & REG_SZ & http://iwww microsoft.com/ & \\
\hline & & $\square$ Settings & abjurl22 & REG_SZ & http://wwww.cia.gov/ & \\
\hline & & $\square$ Toolbar & abjurl23 & REG_SZ & http://WwW.cmu.edu' & \\
\hline & & $\square$ Explorer & abjurl24 & REG_SZ & New Volume (D:) & \\
\hline & & ShellBrowser & abjurl25 & REG_SZ & https://iwww.cmu. edu/myandrew & \\
\hline & & G WebBrowser & abjurl3 & REG_SZ & http://wwww. zoomerang, com/survey $. z g i ? p=$ WEB223 & \\
\hline & & 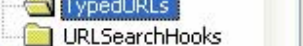 & abjurl4 & REG_SZ & http://www accuweather,com/ & \\
\hline & + & Internet Mail and News & abjurl5 & REG_SZ & http://maps.yahoo.com/ & \\
\hline & + & Internet Services Manage & aburl6 & REG_SZ & http://maps.yahho.com/ & \\
\hline & 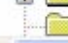 & Java VM & abjurl7 & REG_SZ & http://wwww. homedepot.com/ & $\sim$ \\
\hline$\leqslant$ & & $>$ & $\leqslant$ & & $>$ & \\
\hline
\end{tabular}

Figure 75: The TypedURLs Folder

\subsubsection{UNIX/LinuX}

\section{BIOS Setup}

Generally the boot sector is found in /dev/hda and the file system is found in /dev/hda1. A SCSI formatted drive might refer to this as /dev/sda instead. 


\section{Event/System Logs}

Almost any event on a Linux system can create a log entry. The degree of logging depends on the configuration of the system. 


\section{Persistent Data Types: Temp Files}

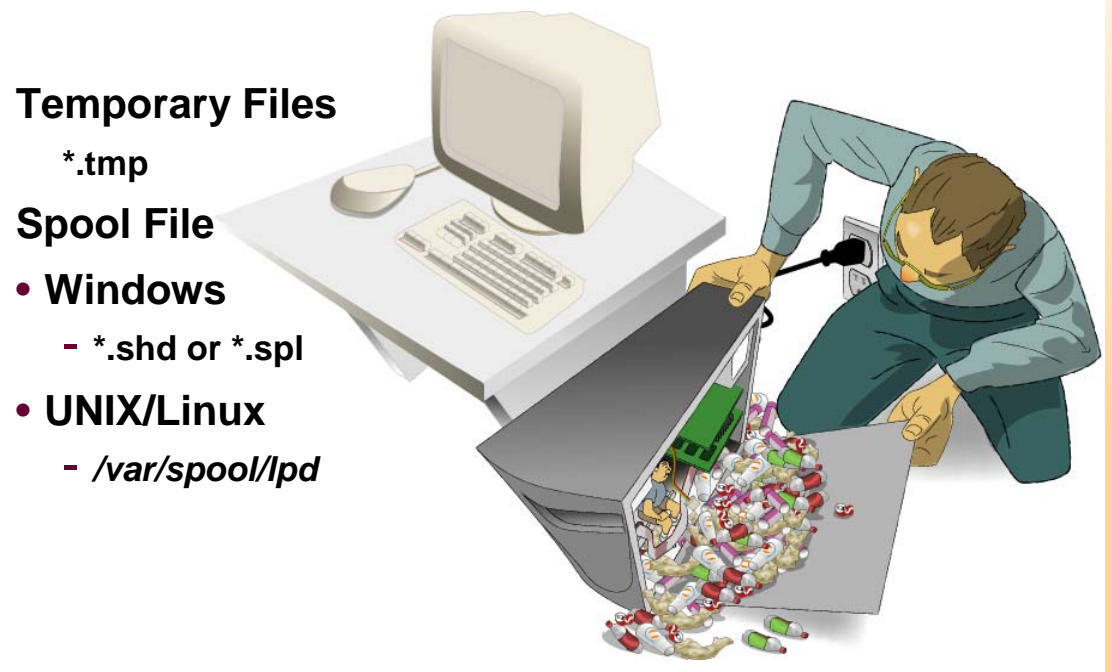

๑) 2005 Carnegie Mellon University

Module 4: Collecting Persistent Data

\subsubsection{Temp Files}

Temp (.tmp) files are created by many applications. Consider Microsoft Word: When you open a document, Word creates a temporary copy of the document to record all changes. When you are finished, Word saves all the recorded changes from the .tmp file into the original file. The .tmp file is then deleted and becomes a part of your hard drive's unallocated space [Kuepper 02].

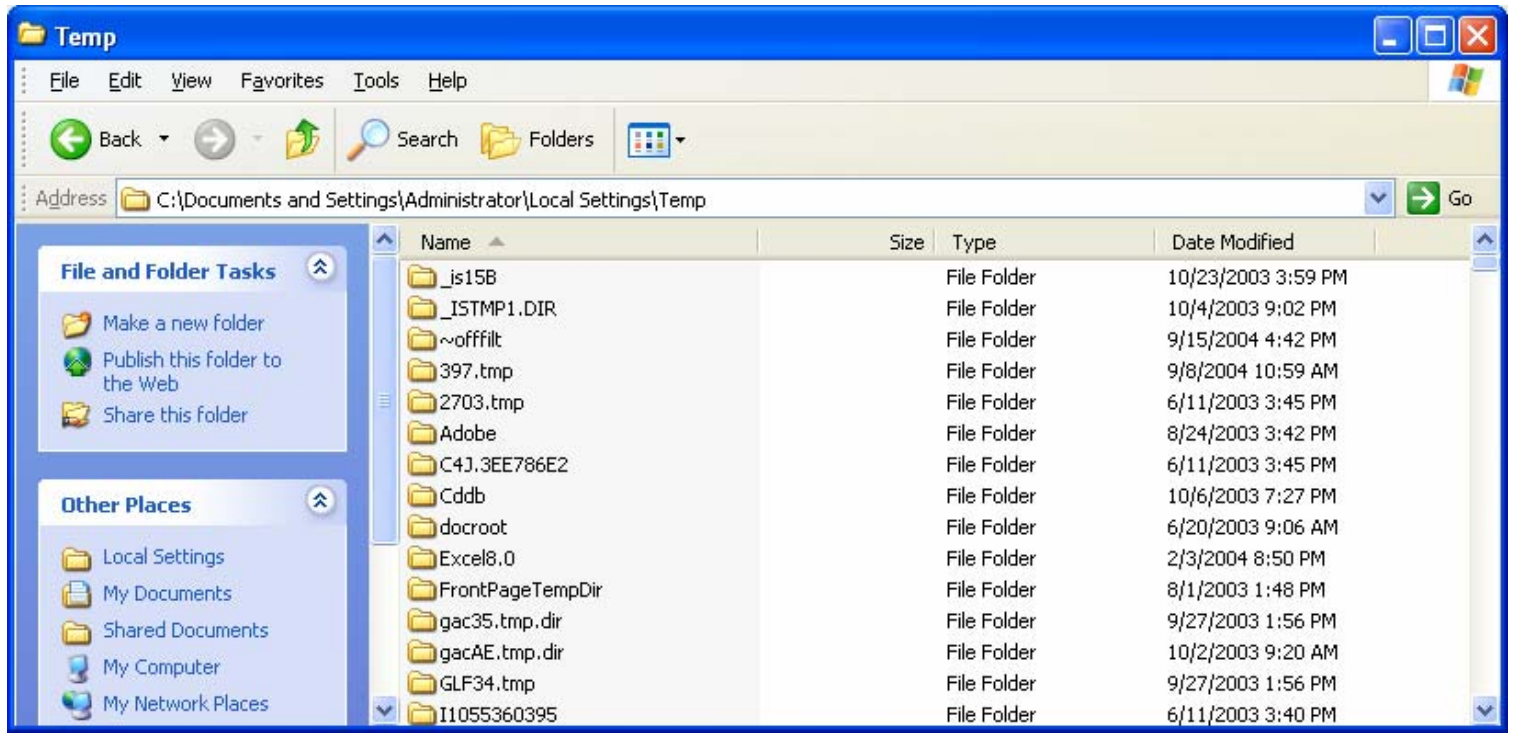

Figure 76: Sample Temp Folder Contents 
Another type of temp file is the spool file. As a job is sent to the printer, the spool file is created to store the information as it waits to be printed. After the job is printed, the disk space that was used by the spool file is reassigned as unallocated, but the printed file is intact until that disk space is overwritten.

Windows creates additional temp files during the printing process as described by Morris [Morris 03]. “These files, which have an .spl or .shd extension, contain a wealth of information about a print job, such as the name of the file printed, the owner of the file, the printer used, and the data to be printed (or a list of the temporary files containing such data).” 


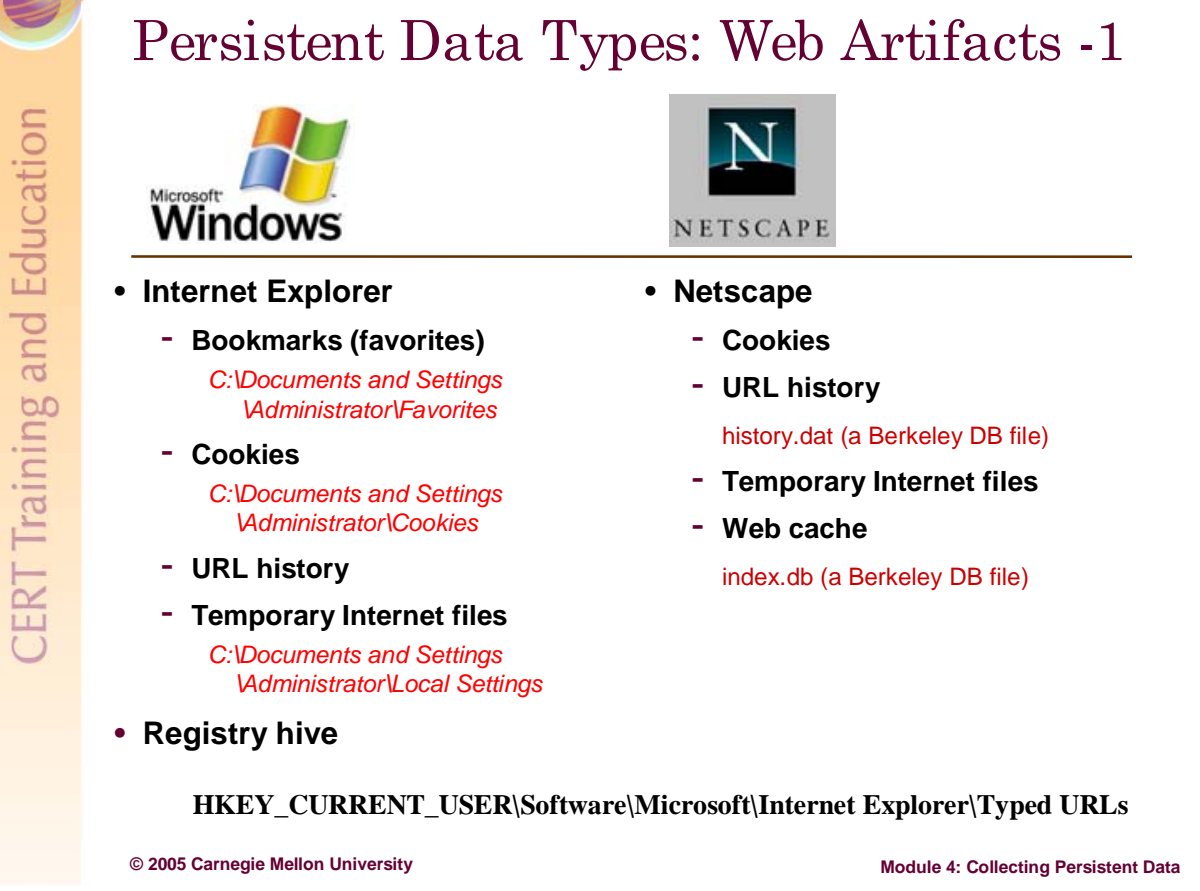

\subsubsection{Web Artifacts}

\subsubsection{Windows vs. Linux}

Web artifact data storage is not as uniform in Linux as it is for IE in Windows. Each Linux flavor, build, or browser might put the data in a slightly different place. Netscape stores the history in Berkeley DB file called history.dat. The cache is stored in another Berkeley DB file, index.db.

Before a security event occurs, then, be sure to document the locations of these files on all flavors of Linux.

In the following section, IE default locations are included with each artifact type.

\subsubsection{IE Default Locations}

\section{Bookmarks}

The IE default location is C:|Documents and Settings|[user]|Favorites. 


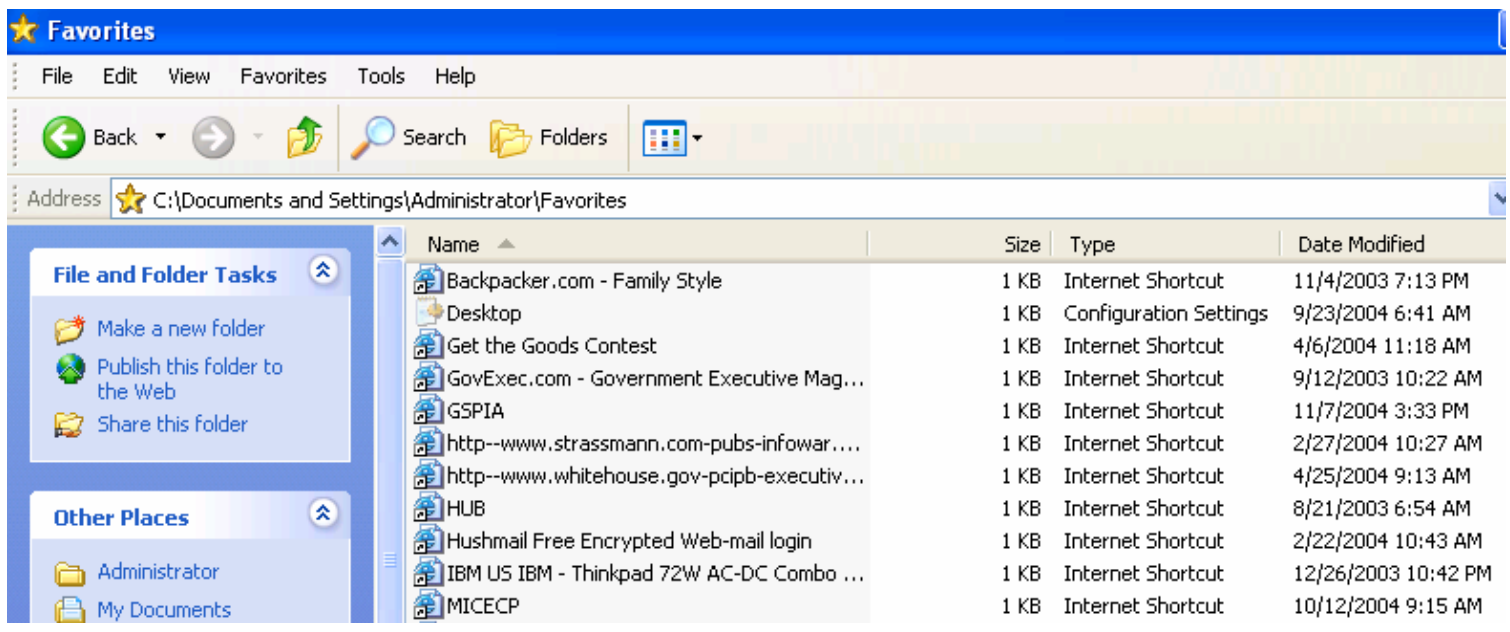

Figure 77: The IE Default Location for Bookmarks

\section{Cookies}

The IE default location is $\mid$ Documents and Settings|[user]|Cookies.

\begin{tabular}{|c|c|c|c|c|c|}
\hline \multicolumn{5}{|l|}{$E$ Cookies } & $-\square x$ \\
\hline File Edit View Favorites & Iools & Help & & & \multirow[t]{2}{*}{ Af } \\
\hline$\theta$ Back $\theta+3$ & & Folders & & & \\
\hline \multicolumn{5}{|c|}{ Address $\square$ C:\{'Documents and Settings $\{$ AdministratoríCookjes } & $\checkmark \rightarrow G_{0}$ \\
\hline \multirow[b]{2}{*}{ File and Folder Tasks } & \multicolumn{2}{|r|}{ ^ Name - } & Size & Type & Date Modified \\
\hline & \multirow{7}{*}{\multicolumn{2}{|c|}{$\begin{array}{l}\text { Ewaits@moneycentral.msn[1] } \\
\text { cwaits@msn.match[1] } \\
\text { cwaits@msn[1] } \\
\text { cwaits@msnbc[2] } \\
\text { cwaits@my.avantgo[2] } \\
\text { cwaits@netflix[2] } \\
\text { cwaits@newsday[1] }\end{array}$}} & $1 \mathrm{~KB}$ & Text Document & \multirow{2}{*}{$\begin{array}{l}9 / 28 / 200411: 30 \mathrm{AR} \\
11 / 11 / 20033: 39 \mathrm{PV}\end{array}$} \\
\hline \multirow{5}{*}{$\begin{array}{l}\text { Make a new folder } \\
\text { Publish this folder to } \\
\text { the Web } \\
\text { Share this folder }\end{array}$} & & & $1 \mathrm{~KB}$ & Text Document & \\
\hline & & & $1 \mathrm{~KB}$ & Text Document & \multirow{2}{*}{ 10/22/2004 10:17 A } \\
\hline & & & $1 \mathrm{~KB}$ & Text Document & \\
\hline & & & $1 \mathrm{~KB}$ & Text Document & $2 / 19 / 200410: 38 \mathrm{PN}$ \\
\hline & & & $1 \mathrm{~KB}$ & Text Document & $2 / 21 / 200412: 13 \mathrm{PN}$ \\
\hline & & & $1 \mathrm{~KB}$ & Text Document & 9/4/20039:45 AM \\
\hline \multirow[t]{2}{*}{ Other Places } & \multicolumn{2}{|r|}{ 首 cwaits@nextag[1] } & $1 \mathrm{~KB}$ & Text Document & $10 / 19 / 200410: 35 \mathrm{P}$ \\
\hline & \multirow{2}{*}{\multicolumn{2}{|c|}{$\begin{array}{l}\text { 眀 cwaits@nytimes[1] } \\
\text { cwaits@office.microsoft[2] }\end{array}$}} & $1 \mathrm{~KB}$ & Text Document & $10 / 22 / 200410: 22 \mathrm{~A}$ \\
\hline Administrator & & & $1 \mathrm{~KB}$ & Text Document & $9 / 21 / 20048: 56 \mathrm{AM}$ \\
\hline & \multicolumn{2}{|r|}{ 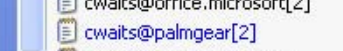 } & $1 \mathrm{~KB}$ & Text Document & $9 / 9 / 20034: 04 \mathrm{PM}$ \\
\hline \multirow{2}{*}{$\square$ Shared Documents } & \multicolumn{2}{|r|}{ 1) cwaits@partner2profit[2] } & $1 \mathrm{~KB}$ & Text Document & $10 / 1 / 20042: 42 \mathrm{PM}$ \\
\hline & \multirow{3}{*}{\multicolumn{2}{|c|}{ 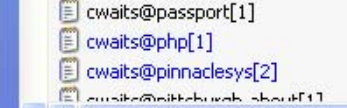 }} & $1 \mathrm{~KB}$ & Text Document & \multirow{3}{*}{$\begin{array}{l}\text { 10/15/2004 9:37 AR } \\
3 / 2 / 2004 \text { 12:30 AM } \\
10 / 8 / 2003 \text { 10:25 AM } \\
\text { O/1 A }\end{array}$} \\
\hline \multirow{3}{*}{ 23 My Network Places } & & & $1 \mathrm{~KB}$ & Text Document & \\
\hline & & & $\begin{array}{l}1 \mathrm{~KB} \\
1 \mathrm{VO}\end{array}$ & Text Document & \\
\hline & \multicolumn{2}{|c|}{$v<$} & & & $\mid>$ \\
\hline
\end{tabular}

\section{Figure 78: The IE Default Location for Cookies}

\section{URL History}

The IE default location is $\mid$ Documents and Settings $\mid$ [user] Local Settings $\mid$ History. 


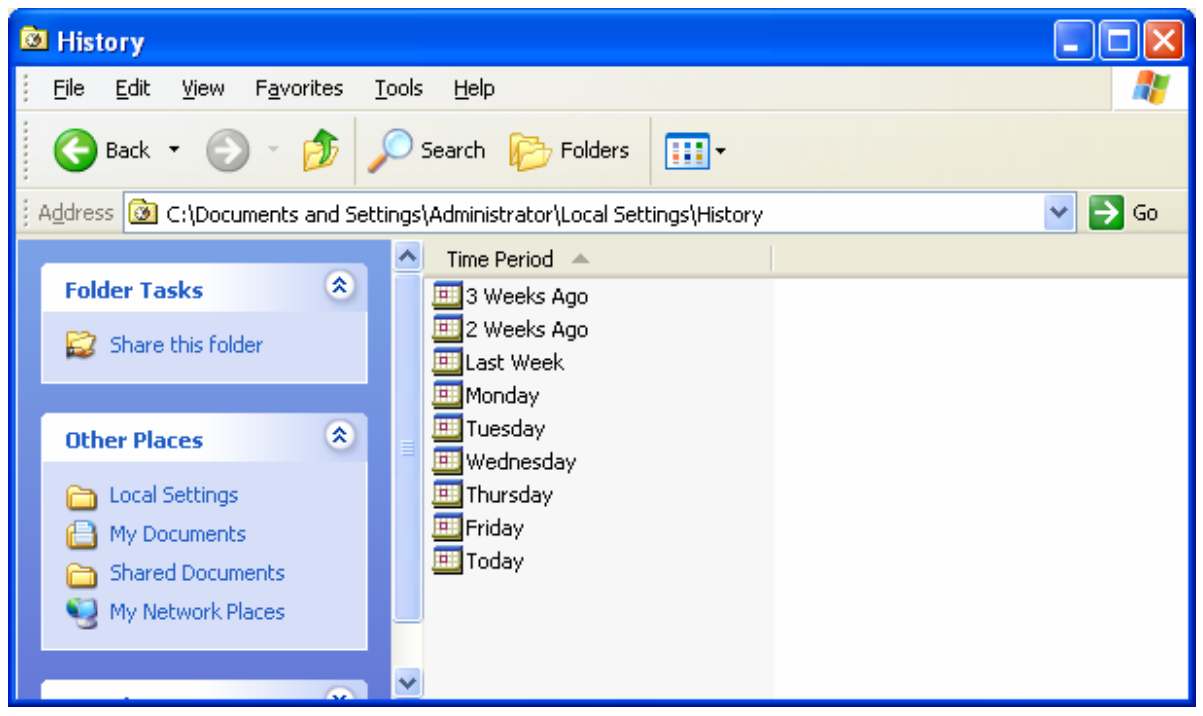

Figure 79: The IE Default Location for URL History

\section{Temporary Internet Files/Web Cache}

The contents of each Web page you visit are downloaded to files. Even after these files are deleted, information is left in the unallocated space.

The IE default location is $\mid$ Documents and Settings $\mid$ [user] $\mid$ Local Settings|Temporary Internet Files.

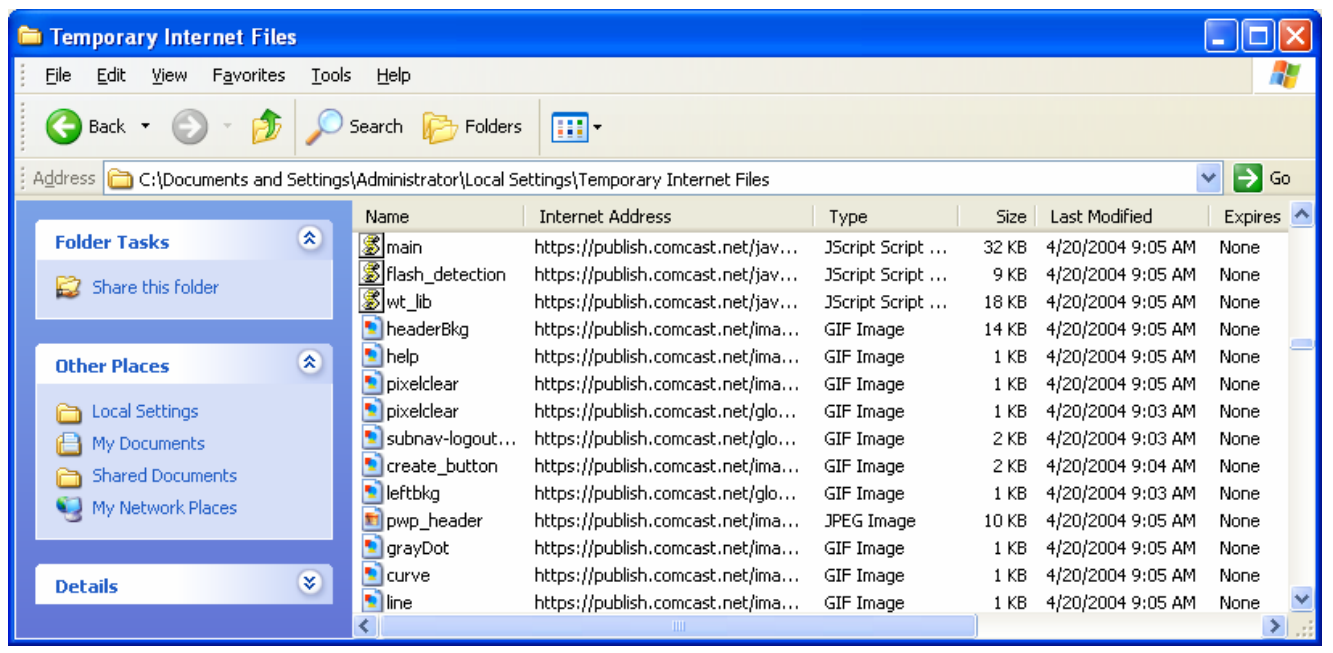

Figure 80: The IE Default Location for Web Cache

\section{Registry Hive}

The registry HKEY_CURRENT_USER\Software\Microsoft|Internet Explorer\Typed URLs contains a record of the URLs typed into IE. If a user goes to the history file and deletes entries, those URLs may still be in the registry hive. However, if a user clicks the "Clear 
History" button in the Tools > Internet Options > "General" tab of IE, the entries in the hive will be cleared out.

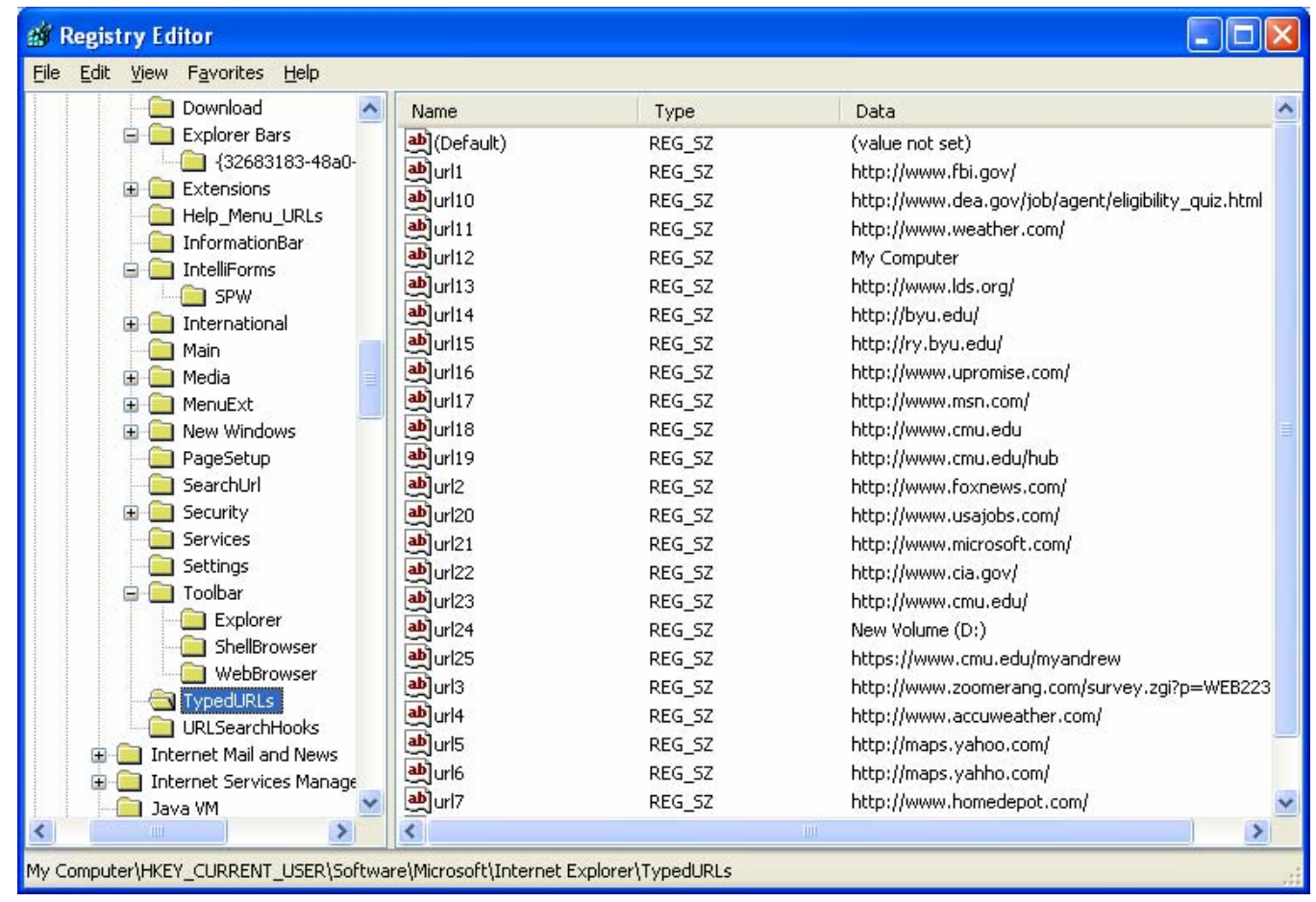

\subsubsection{Alternative Browsers}

Remember that though we live in a Windows universe, there are other browsers. The cookies, history, cache, and temp files for the Web activity performed using these other browsers are not stored in the Windows locations. Where you find this data depends on how the browser was installed. For instance, the Mozilla browser installed on my computer stores the cookies, history, and cache in the C: $\mid$ Documents and Settings\Administrator|Application Data $\mid$ Mozilla|Profilesidefault|wq809fmw.slt folder. 


\section{Persistent Data Types: Web Artifacts -3}

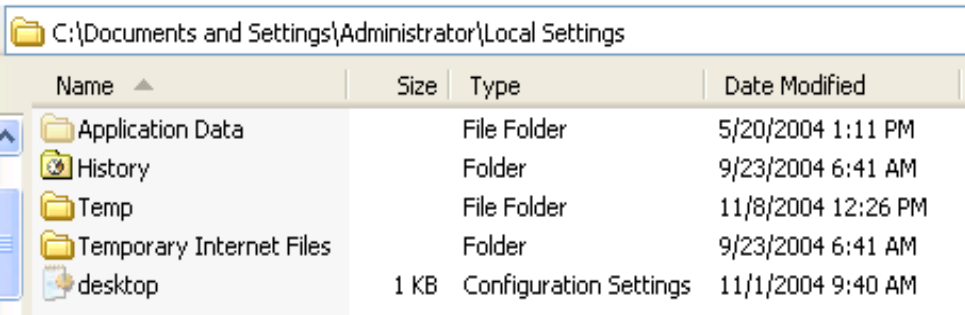

(c) 2005 Carnegie Mellon University

Module 4: Collecting Persistent Data

\subsubsection{Cookies}

Often, a Web site gathers information about a visitor's habits by placing a cookie on the user's computer. These can be used for anything from keeping track of user's preferred book genre in order to make better recommendations to remembering the user's zip code so they can provide local weather reports.

Cookies can also be used for nefarious purposes. Malicious Web sites can upload malware masquerading as a cookie to a user's computer. For more information on cookies and their uses, visit http://www.cookiecentral.com/. 


\section{Persistent Data Types: File Recovery}

\section{Document types}

- Files

- Emails

\section{Slack space}

- RAM slack

- Drive slack

Swap files

Unallocated space

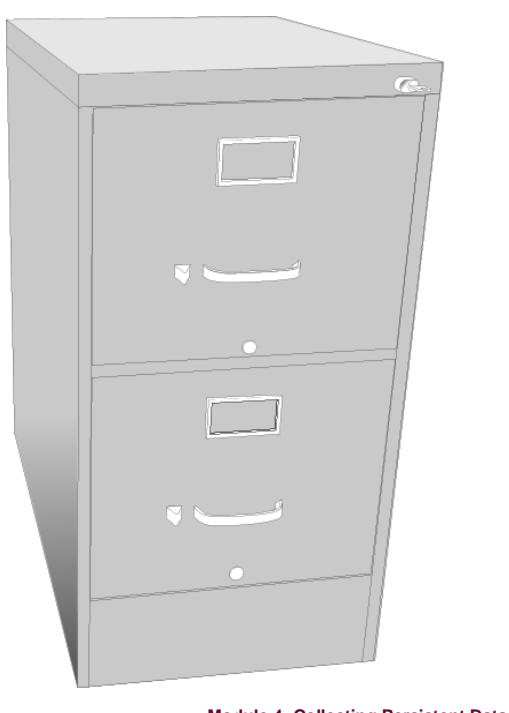

Module 4: Collecting Persistent Data

\subsubsection{File Recovery}

While there are some differences, Windows and Linux file recovery is similar.

\subsubsection{Deleted Data}

There are several different ways to delete data in Windows.

Files can be deleted using Windows Explorer, which is the most common method. The deleted file is put into the Recycle Bin but can be easily restored because, per Nelson [Nelson 04], Windows first "changes the name of the file and moves it to a subdirectory with a unique identity in the Recycle Bin.” Info2, the control file for the Recycle Bin, contains information about the original path and file name.

Files can be deleted using the MS-DOS functions.

Files can be "wiped" using a variety of special software tools. Remember from Module 2 that wiping is different than deleting because clusters where the data was stored are written over to prevent their recovery from slack or unallocated space.

Deleting a file, however, does not remove its short cut from the Recent Files list. These files, which are not available through normal means, are available through a forensic examination of the slack or unallocated space.

Not all flavors of Linux make use of a Recycle bin the way Windows does. When deleted on a UNIX system, Casey [Casey 00] says "the file's directory entry is hidden from view and the 
system notes that the associated inode is available for reuse. The file's directory entry, inode, and data remain on the disk until they are overwritten.”

\subsubsection{Slack Space}

Logical file size: Actual size of the file

Physical file size: Amount of space a file takes up on the disk, given in whole number of clusters

Sector slack: Left over capacity in a sector, filled with data held in the RAM

Drive slack: Excess capacity in a cluster, empty or filled with whatever was previously stored in that series of consecutive sectors

\subsubsection{Swap Files}

Windows swap files can be temporary or permanent, depending on which Microsoft OS you have.

In Windows 95, 98, and Me, the temporary swap file expands and contracts depending on how much space it needs. To run multiple programs simultaneously with limited RAM, Windows uses this swap file to expand the computer's RAM. Windows swaps an application's data to and from the swap file as needed, based on what is actively running. Because this file contains application data, there could be almost any type of data in it: passwords, complete or partial documents, email, credit card numbers, images, and so on.

In Windows NT, 2000, and XP, the permanent swap file does not expand and contract. By default, Windows 2000 sets the swap size to the amount of RAM * 1.5.

As a side note, there is a local security policy setting under Start $>$ Programs $>$ Administrative Tools > Local Security Policies > Local Policies > Security Options that allows you to set Windows to "Clear virtual memory page when Windows shuts down" [Kuepper 02].

\subsubsection{Unallocated Space}

Unallocated space are portions of the drive that are not actively assigned data.

\subsubsection{Partial Files}

At times, the file system begins writing a file to a disk or partition and does not realize that the target space can not contain the entire file until it has filled all the available space. This generally results in an error and a request that the user clear some space. Partial files do not show up in the directory but remain in unallocated space until they are overwritten. 


\subsubsection{Windows Artifacts}

Windows artifacts, which are created by the Windows OS, are important for forensic

examiners as they attempt to recreate a user's activities—partly because the artifacts are often overlooked by users or intruders as they attempt to cover their tracks. They include files used as shortcuts to applications, files, or devices, or certain files created by the OS. Examples are the .lnk files created in the Windows Desktop, Recent, Send To, and Start menu folders.

Examine these artifacts to uncover files, folders, applications, or devices that are no longer on the system. For example, a forensic examiner may discover links to files that have been deleted and are no longer recoverable or that were stored on a network drive. Links to other storage devices (i.e., Zip or Jazz drive) may indicate that other media need to be located and analyzed [Morris 03]. 


\section{Persistent Data Types: Hidden Files}

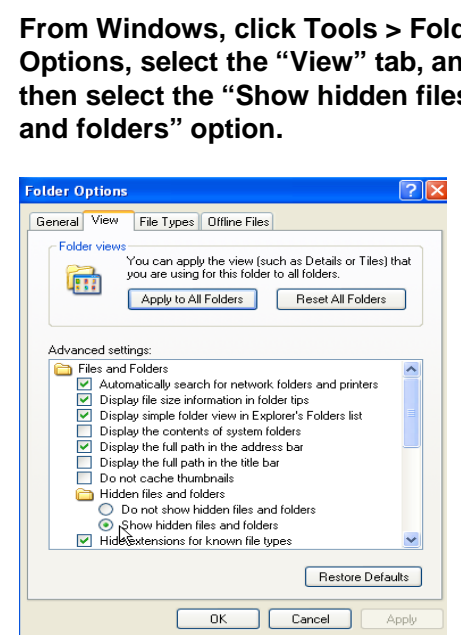

๑ 2005 Carnegie Mellon University

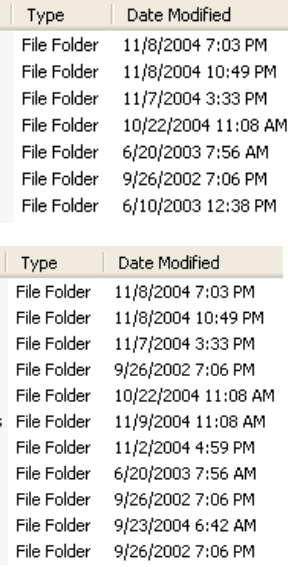

Module 4: Collecting Persistent Data

\subsubsection{Hidden Files}

Files can be assigned an attribute so that they will not be displayed with normal file system viewing methods (i.e., Windows Explorer and the DOS dir command ). Files may be marked as hidden to protect files from being corrupted by casual users or to hide illicit data or activities.

To view hidden files with Windows Explorer, go to Tools > Folder Options, click the "View" tab, and select the Show Hidden files option. 


\section{Recovering a Deleted Email}

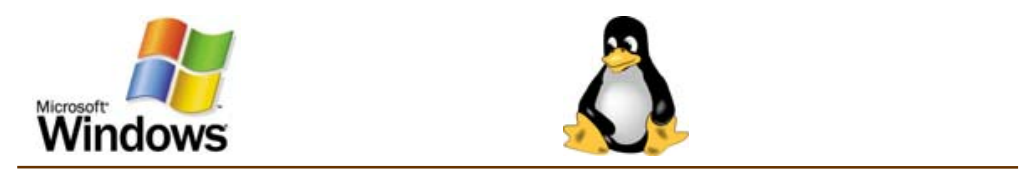

-.pst files

C:IDocumentsandSettings| Administratorl

LocalSettings/ApplicationData IMicrosoftlOutlook
- Incoming

-/var/spool/mail

- Outgoing

-/var/spool/mqueue/mail

\subsection{Recovering a Deleted Email}

Windows Outlook emails are .pst files. Their default location is $\mid$ Documents and Settings \[user]|Local Settings\Application Data|Microsoft $\mid$ Outlook.

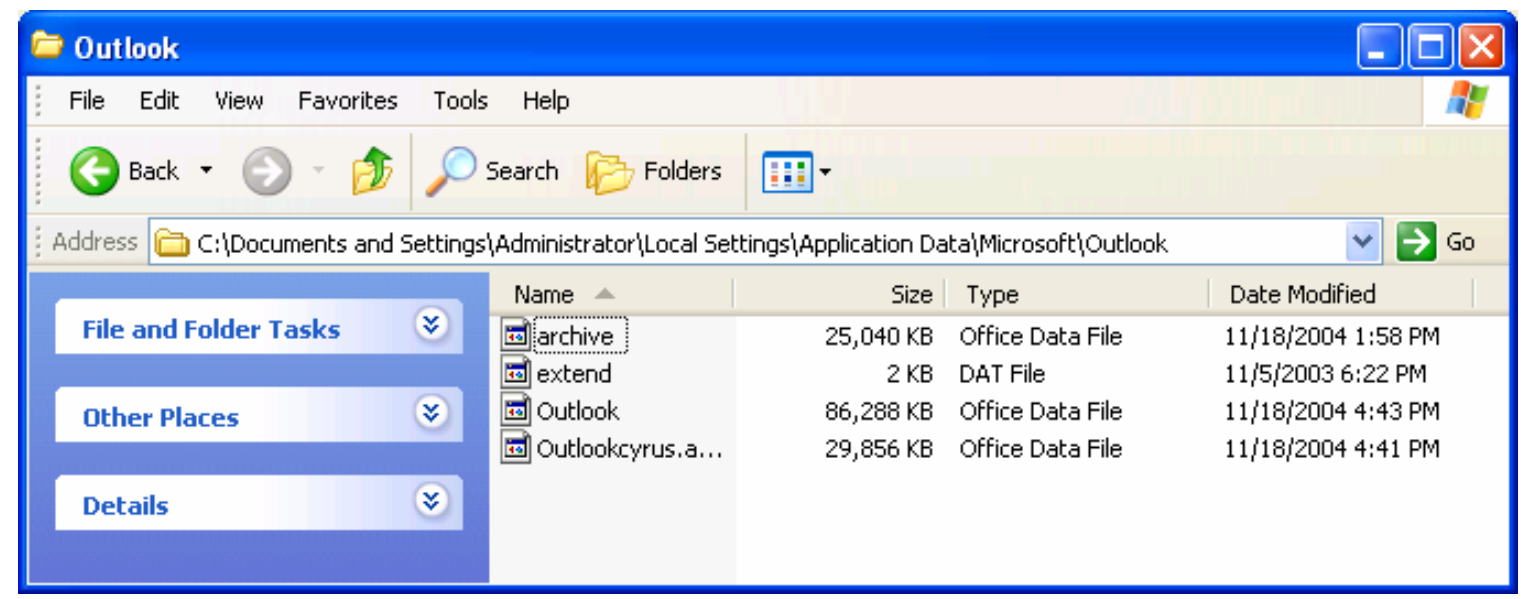

Figure 81: The Default Location for Outlook Email Files

Because the .pst files are in a proprietary Windows format, they are more easily viewed with Outlook or Outlook Express. To view them, copy the .pst files from the suspicious computer to another computer with Outlook. 


\section{Tools for Accessing Persistent Data}

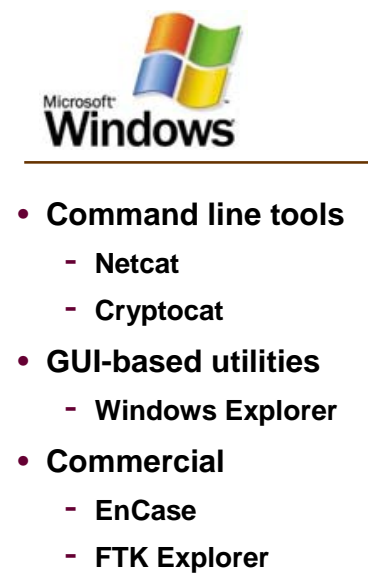

- Freeware

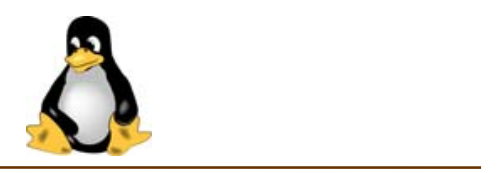

- Command line tools

- Sleuth Kit

- GUI-based utilities

- Autopsy Forensic Browser

- Commercial

- Freeware

- The Coroner's Toolkit

- Sleuth Kit

- KNOPPIX STD

๑ 2005 Carnegie Mellon University

Module 4: Collecting Persistent Data

\subsection{Tools for Accessing Persistent Data}

There is a lot of overlap in the forensic tool market. The most important quality to look for is usability. If the tool works for you and it meets admissibility standards, select it.

Command-line tools are generally small and portable. They fit easily onto a floppy disk or CD-ROM and if packaged correctly can be used safely on a live suspicious computer.

Commercial tools are more polished and often more steamlined. They perform the same tasks as the freeware tools, but what might take 13 steps with a free product will only take two steps with a commercial product.

What follows is a small sample of the forensic tools available.

\subsubsection{Windows}

\subsubsection{Command-Line Tools}

- Netcat

- dd

Download this tool from http://uranus.it.swin.edu.au/ jn/linux/rawwrite/dd.htm.

\subsubsection{GUI-Based Utilities}

- Windows Explorer 
Explorer is native to the Windows OS. It allows you to navigate through the file systems of a mounted image drive.

\subsubsection{Commercial}

- EnCase

EnCase is a full service tool that handles acquisition and analysis of volatile and nonvolatile data.

For more details, visit http://www.guidancesoftware.com/products/index.shtm.

- FTK (Forensic Tool Kit)

FTK is an all-in-one collection and examination tool.

For more details, visit

http://www.accessdata.com/Product04_Overview.htm?ProductNum=04.

\subsubsection{UNIX/LinuX}

\subsubsection{Command-Line Tools}

- Netcat

Netcat is a networking utility that reads and writes data across network connections using the TCP/IP protocol.

For more details, visit http://netcat.sourceforge.net.

- dd

This tools creates drive or partition images. A version of this tool has also been developed for Windows. Most versions of Linux have $d d$ as a native tool.

\subsubsection{GUI-Based Utilities}

- Autopsy Forensic Browser

The Autopsy Forensic Browser is a graphical interface to the command-line digital forensic analysis tool, The Sleuth Kit. Together, The Sleuth Kit and Autopsy provide many of the same features as commercial digital forensics tools for the analysis of Windows and UNIX file systems (NTFS, FAT, FFS, ext2, and ext3).

For more details, visit http://www.sleuthkit.org/autopsy

\subsubsection{Freeware}

- The Sleuth Kit

The Sleuth Kit (previously known as TASK) is a collection of UNIX-based commandline file system and media management forensic analysis tools. The file system tools 
allow you to examine a suspicious computer in a non-intrusive fashion. Because the tools do not rely on the OS to process the file systems, deleted and hidden content is shown.

For more details, visit http://www.sleuthkit.org/sleuthkit/desc.php.

- $\quad$ The Coroner’s Toolkit

The Coroner' Toolkit (TCT) is a collection of tools that gather and analyze data on a UNIX. unrm and lazarus are part of the toolkit and can be used to restore deleted files and data that cannot be easily accessed.

For more details, visit http://www.fish.com/tct.

- Knoppix STD

Knoppix-STD is a customized distribution of the Knoppix Live Linux CD. Boot to the CD and you have Knoppix-STD. STD focuses on information security and network management tools.

For more details, visit http://www.knoppix-std.org. 


\section{Summary}

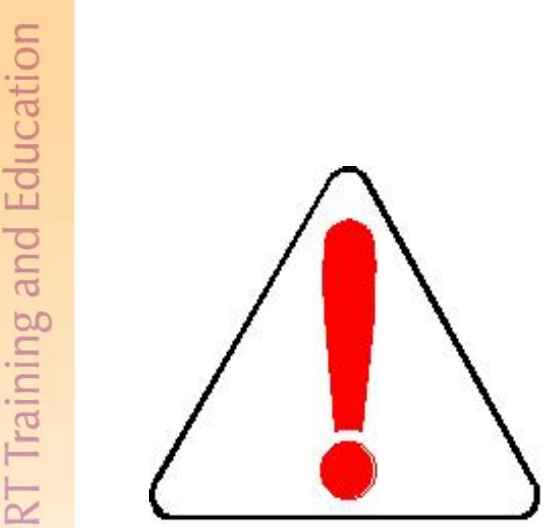

๑) 2005 Carnegie Mellon University
Persistent data survives after the power is turned off.

Improper collection techniques cause corruption.

Each OS will handle data differently.

Establish procedures before you need to use them.

\subsection{Summary}

It is important to remember at least four points from this module:

1. Persistent data survives after the power is turned off.

2. Improper collection techniques cause corruption. "Primum non nocere" or "First do no harm” could be the dictum when collecting persistent data.

3. Each OS will handle data differently, so be familiar with the computers on your network.

4. Establish procedures before you need them.

Performing an ad hoc investigation leads to unnecessary aggravation. There will be enough aggravation even with proper preparation. 


\section{Review}

\section{What is persistent data?}

2. How can persistent data be compromised?

3. List three types of persistent data.

4. Should the computer be shut down before collecting nonvolatile data?

\section{List the basic steps of collecting forensically sound data.}

\subsection{Review}

1. Persistent (or nonvolatile) data retains its state after the power has been turned off.

2. Compromised persistent data can result from just about any action taken on the host computer. Browsing the Web, opening files, and even creating new files can compromise persistent data.

3. Types of persistent data include deleted files, cookies, Web history, MAC times, registry hives, emails, recycle bin, and recent document shortcuts.

4. The computer should not necessarily be shut down before collecting nonvolatile data. It is always easier to collect persistent data from a dead hard drive mounted on a forensic workstation, but circumstances dictate the appropriate course of action. Sometimes a computer must be left on. In these cases there is nothing to do but collect from the live computer.

5. Steps for collecting forensically sound data include (1) have a pre-established procedure in place; (2) document steps taken; (3) image the suspicious computer's hard drive; (4) establish the integrity of the image using a MD5 hash; (5) perform any analysis of working copies. 


\section{References}

URLs are valid as of the publication date of this document.

[ACPO 03]

[Barrett 04]

[Brezinski 02]

[CAE 04]

[Carvey 04]

[Casey 00]

[CDT 04]

[GNU 04]

[Grundy 04]

[Haas 04]
Association of Chief Police Officers (ACPO). Good Practice Guide for Computer based Electronic Evidence. http://www.4law.co.il/ Lea92.htm (2003).

Barrett, Daniel J. Linux Pocket Guide. Sebastopol, CA: O’Reilly, 2004.

Brezinski, D. Guidelines for Evidence Collection and Archiving (Network Working Group RFC 3227). http://www.ietf.org/rfc/ rfc3227.txt (2002).

Computer-Aided Engineering Center, University of WisconsinMadison, College of Engineering. Linux File Permissions Overview. http://www.cae.wisc.edu/fsg/linux/linux-filepermissions.shtml (2004).

Carvey, Harlan. Windows Forensics and Incident Recovery. Boston, MA: Addison-Wesley, 2004 (ISBN 0-321-20098-5).

Casey, Eoghan. Digital Evidence and Computer Crime, Second Edition. San Diego, CA: Academic Press, 2000 (ISBN 0-121-62885$\mathrm{X})$.

Center for Democracy and Technology. Impact of the McCain-Kerrey Bill on Constitutional Privacy Rights. http://www.cdt.org/crypto/ legis_105/mccain_kerrey/const_impact.html (2004).

The GNU Netcat Project. What is Netcat? http://netcat.sourceforge.net (2004).

Grundy, Barry J. The Law Enforcement and Forensic Examiner Introduction to Linux: A Beginner's Guide, Ver 2.0.5. http://www.linux-forensics.com/linuxintro-LEFE-2.0.5.pdf (2004).

Haas, Juergen. Linux / Unix Command: checkconfig. http://linux.about.com/library/cmd/blcmdl8_chkconfig.htm (2004). 
[Honeynet 04]

[ICH 03]

[Kerr 01]

[Kuepper 02]

[Mandia 01]

[Microsoft 03a]

[Microsoft 03b]

[Microsoft 04]

[MIT 03]

[Morris 03]

[Nelson 04]

[Nemeth 01]

[NIJ 04]
The Honeynet Project. Know Your Enemy: Learning About Security Threats, Second Edition. Boston, MA: Pearson Education, Inc., 2004 (ISBN 0-321-16646-9).

ICH Architecture Resource Center. Interoperability Clearinghouse Glossary of Terms. www.ichnet.org/glossary.htm (2003).

Kerr, Orin S. "Computer Records and the Federal Rules of Evidence.” USA Bulletin 49, 2 (March 2001): 25-32.

http://www.usdoj.gov/usao/eousa/foia_reading_room/usab4902.pdf

Kuepper, Brian. What You Don't See on Your Hard Drive. http://www.sans.org/rr/whitepapers/incident/653.php (2002).

Mandia, Kevin \& Prosise, Chris. Incident Response: Investigating Computer Crime. Berkeley, CA: Osborne/McGraw-Hill, 2001.

Microsoft. FAT File System Technology and Patent License. http://www.microsoft.com/mscorp/ip/tech/fat.asp (2003).

Microsoft. FAT File System: The Story Behind the Innovation. http://www.microsoft.com/mscorp/ip/tech/fathist.asp (2003).

Microsoft. Microsoft $®$ Windows $®$ XP Professional Resource Documentation Kit.

http://www.microsoft.com/resources/documentation/

Windows/XP/all/reskit/en-us (2004).

Massachusetts Institute of Technology. MIT researchers uncover mountains of private data on discarded computers. http://web.mit.edu/newsoffice/2003/diskdrives.html (2003).

Morris, Jamie. Forensics on the Windows Platform, Part Two. http://www.securityfocus.com/infocus/1665 (2003).

Nelson, Bill. Guide to Computer Forensics and Investigations. Boston, MA: Thomson Course Technology, 2004 (ISBN 0-61913120-9).

Nemeth, Evi. UNIX System Administration Handbook, $3^{\text {rd }}$ Edition. Upper Saddle River, NJ: Prentice Hall PTR, 2001 (ISBN 0-13020601-6).

National Institute of Justice. Forensic Examination of Digital Evidence: A Guide for Law Enforcement. http://www.ncjrs.org/pdffiles1/nij/199408.pdf (2004). 
[NIST 01]

[NIST 03]

[NTI 03]

[Pierce 03]

[Russinovich 97]

[Russinovich 00]

[Russinovich 01]

[Russinovich 03]

[Russinovich 04a]

[Russinovich 04b]

[Russinovich 04c]

[Russinovich 04d]

[Sorenson 03]

[Stoffregen 03]
Wack, John P. Establishing a Computer Security Incident Response Capability. http://csrc.nist.gov/publications/nistpubs/800-3/800-3.pdf (2001).

National Institute of Standards and Technology. CFTT Metholology Overview. http://www.cftt.nist.gov/Methodology_Overview.htm (2003).

New Technologies, Inc. Computer Forensics Definitions. http://www.forensics-intl.com/define.html (2003).

Pierce, Matt. Detailed Forensic Procedure for Laptop Computers. http://www.giac.org/practical/GSEC/Matt_Pierce_GSEC.pdf (2003).

Russinovich, Mark. Inside the Registry.

http:/www.microsoft.com/technet/prodtechnol/winntas/tips/ winntmag/inreg.mspx (1997).

Russinovich, Mark. ListDDLs. http://www.sysinternals.com/ ntw2k/freeware/listdlls.shtml (2000).

Russinovich, Mark. PsFile. http://www.sysinternals.com/ ntw2k/freeware/psfile.shtml (2001).

Russinovich, Mark. PsService. http://www.sysinternals.com/ntw2k/ freeware/psservice.shtml (2003).

Russinovich, Mark. PsInfo. http://www.sysinternals.com/ntw2k/ freeware/psinfo.shtml (2004).

Russinovich, Mark \& Cogswell, Bryce. Autoruns. http://www.sysinternals.com/ntw2k/freeware/autoruns.shtml (2004).

Russinovich, Mark. Handle. http://www.sysinternals.com/ntw2k/ freeware/handle.shtml (2004).

Russinovich, Mark. Filemon for Windows. http://www.sysinternals.com/ntw2k/source/filemon.shtml (2004).

Sorenson, Holt. Incident Response Tools For Unix, Part One: System Tools. http://www.securityfocus.com/infocus/1679 (2003).

Stoffregen, Paul. Understanding FAT32 Filesystems. http://www.pjrc.com/tech/8051/ide/fat32.html (2003). 
[SystemTools 04]

[Uniblue 04]

[USDOJ 02]

[Vidstrom 04]

[Wheeler 03]

[Wikipedia 04]
SystemTools.com. SomarSoft Utilities. (Click Free Tools.) http://www.somarsoft.com (2004).

Uniblue. WinTasks Process Library. http://www.liutilities.com/ products/wintaskspro/processlibrary (2004).

United States Department of Justice. Searching and Seizing Computers and Obtaining Electronic Evidence in Criminal Investigations. http://www.cybercrime.gov/s\&smanual2002.htm (2002).

Vidstrom, Arne. NETSECURITY.NU. http://www.ntsecurity.nu/ toolbox/macmatch (2004).

Wheeler, David A. Program Library HOWTO. http://www.linux.com/ howtos/Program-Library-HOWTO/index.shtml (2003).

Wikipedia's Library (computer science). http://www.factindex.com/ l/li/library_computer_science_html (2004). 


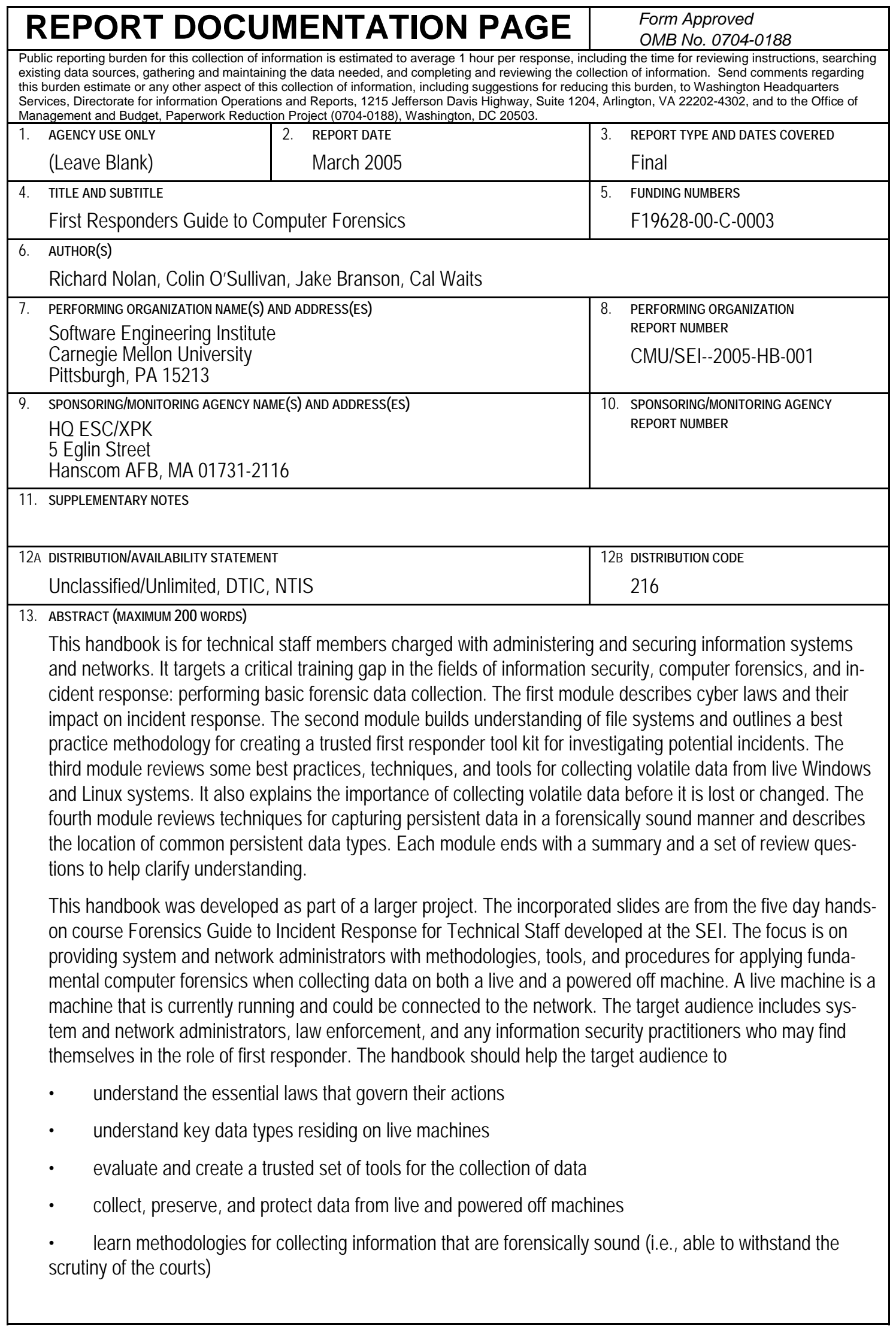




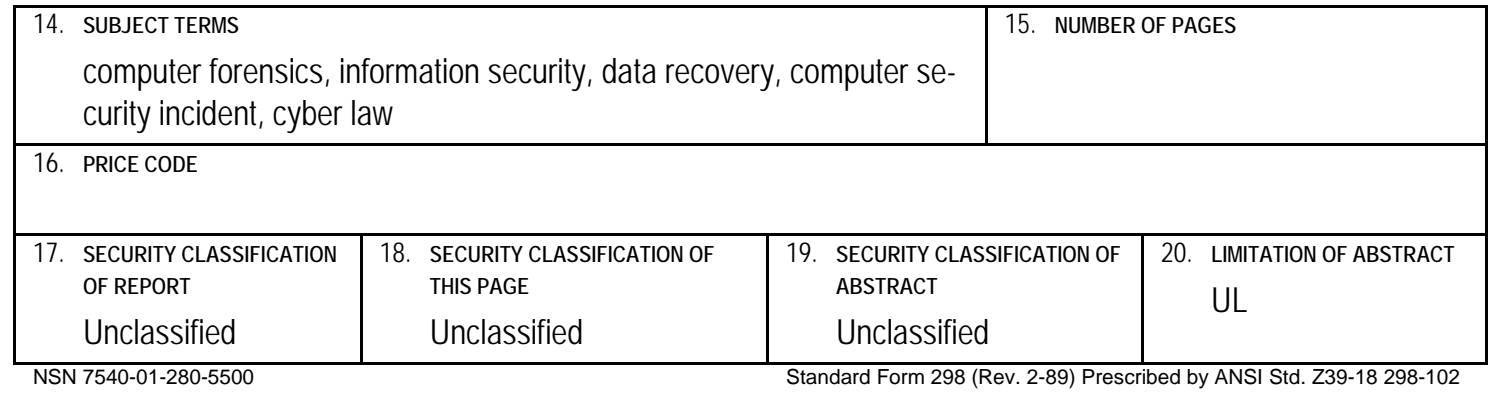

